\section{ACOUSTIC COMMUNICATION IN THE NORTH ATLANTIC RIGHT WHALE (Eubalaena glacialis)}

By

Susan Elizabeth Parks

B. A., Cornell University, 1998

Submitted in partial fulfillment of the requirements for the degree of

Doctor of Philosophy

at the

MASSACHUSETTS INSTITUTE OF TECHNOLOGY

and the

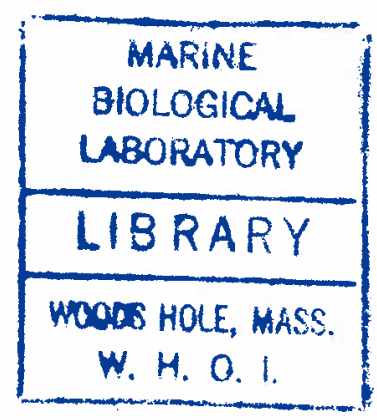

WOODS HOLE OCEANOGRAPHIC INSTITUTION

September 2003

(C) 2003 Susan Elizabeth Parks. All rights reserved.

The author hereby grants to MIT and WHOI permission to reproduce paper and electronic copies of this thesis in whole or in part and to distribute them publicly.

Signature of Author

Joint Program in Oceanography/Applied Ocean Science and Engineering Massachusetts Institute of Technology and Woods Hole Oceanographic Institution September 2003

Certified by Peter Lloyd Tyack Thesis Supervisor

Accepted by

John B. Waterbury Chair, J $\phi$ init Committee for Biological Oceanography Massachusetts Institute of Technology/ Woods Hole Oceanographic Institution 


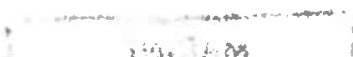

$$
\begin{aligned}
& \text { isind in }
\end{aligned}
$$

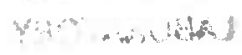

$$
\begin{aligned}
& \text { (1) } \\
& A+3 ! 1
\end{aligned}
$$

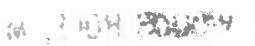

$$
\begin{aligned}
& 6 \div
\end{aligned}
$$




\title{
ACOUSTIC COMMUNICATION IN THE NORTH ATLANTIC RIGHT WHALE (Eublaena glacialis)
}

\author{
By Susan Elizabeth Parks
}

Submitted in partial fulfillment of the requirements for the degree of Doctor of Philosophy at the Massachusetts Institute of Technology and the Woods Hole Oceanographic Institution.

\section{Abstract}

The focus of this thesis is the use of sound for communication by the North Atlantic right whale (Eubalaena glacialis). The surface active group (SAG) is the predominant social interaction in this species for which use of sound has been documented. Different group compositions in SAGs indicate that both potentially reproductive and non-reproductive groups have been combined under one label. Sound production in SAGs suggests that females form and maintain the groups by producing Scream calls. Males produce Upcalls to advertise their presence as they come into a group or when the female is on a dive. Males may use Gunshot sounds as threat signals to other males in the group or potentially as reproductive advertisement signals to the female. Some calves produce Warble sounds in SAGs. This may be limited to female calves. This description of acoustic activity in the groups adds to the picture of the SAGs as complex interactions between individuals, rather than simple groups with only one whale producing all the sounds to attract other whales to the group. Playback experiments demonstrate that right whales can use sounds from SAGs to locate the groups. Male right whales approached both North Atlantic and Southern right whale SAG playbacks. Female right whales only approached Southern right whale playbacks. Anatomical modeling resulted in a frequency range of hearing for the right whale $(10 \mathrm{~Hz}-22 \mathrm{kHz})$ that is consistent with the sounds that they produce and overlaps the frequency range of most anthropogenic noise sources. This combination of research provides a thorough description how North Atlantic right whales use sound in SAGs and how increasing levels of noise in the oceans may impact right whales in these groups. 
This thesis is dedicated to the memory of Jackie Ciano

Mentor, teacher, fishing partner and friend. She is sorely missed.

Where a slate grey Bay meets a mackerel sky petrels dance on the water

Batd eagles bracing spindle pines watch over Fundy's daughter When the leaves are turning eiders form great shoals upon the water The fog descends, though then unseen embracing Fundy's Daughter.

Deep currents foed this awesome tide as right whates churn the water Their haunting woices can't compare to that of Fundy's daughter. Where the sun ignites great wheels of life and sparkles on the water. No man made beacom shinesso bright as the light off Fundy's Daughter.

Fundy's Daughter - by Jackie Ciano 


\section{ACKNOWLEDGEMENTS}

There are more people to thank for their assistance in this work than there are pages in the thesis. Every aspect of this thesis work brought me into contact with wonderful, talented people, everyone one of whom I feel privileged to have met. Forgive me if your name is left off.

First I have to thank my parents, Tom and Martha, for their love and support for the past 27 years. None of this would have been possible without their guidance and I thank them for giving me the motivation to undertake this project and the faith to let me stumble through it own my own.

Kurt Fristrup has been a mentor and friend to me for over eight years. His teaching, patience, enthusiasm and support got me to the point I am at today. Thank you for believing in me, even when I don't believe in myself.

Peter Tyack has been a fabulous advisor and cheering squad since the very first day I met him. His knowledge and patience overwhelm me, particularly in the last few months, when he has read drafts of this thesis many times. Discussions with Peter have helped form many of the ideas behind this work and he is responsible for starting me down the path of right whale research. I hope to have many more such discussions with him in the future.

Members of the Tyack lab have provided invaluable support to me over the years. Thank you to Nicoletta Biassoni, Krill Carson, Debby Fripp, Vincent Janik, Patrick Miller, Jen Miksis, Douglas Nowacek, Amy Samuels, Ari Shapiro, Rebecca Thomas, and Stephanie Watwood for being there (and for putting up with me). Special thanks to Patrick Miller for helping me build one of his arrays for this research and to Douglas Nowacek for the experience that was Bay of Fundy 1999-2001. I've learned so much from you all.

Many people assisted me with data collection. I would like to thank everyone working at the New England Aquarium Right Whale Research group over the years. Everything I know about right whales, I learned from you. Scott Kraus, Amy Knowlton, Philip Hamilton, Lisa Conger, Jackie Ciano, Chris Slay, Carolyn Miller, Michael Moore, Brenna Kraus, Jessica Damon, Heather Pettis, Beth Pike, Stephanie Martin, Moe Brown, Owen Nichols, Ingrid Biedron, Tim Fraiser and the rest of the gang made summers in Lubec, ME very special to me. The entire Ketten lab helped me learn the ropes of anatomy. Thank you to Scott Cramer, Jen O’Malley, Julie Arruda, Judy Fenwick, Sarah Marsh and Iris Fischer for all their help and patience. My old friends back at the nest of the Cornell Bioacoustics Research Program including Robert MacCurdy, Connie Gordon, Melissa Fowler, Kathy Dunsmore, Russ Charif and many more have continued to support and teach me, even after I moved to Cape Cod. 
I have made numerous friends here over the years, and they have made my life so much more enjoyable for having known them. Special thanks to Emily Lilly for being my friend, mentor and counselor for the past three years. What would I have done with out you (and the garden)? David Adams, Meredith Canode, Steve Fries, Craig Herbold, Chanda Herring, Rob Jennings, Astri Kvassnes and Andrew Sweetman have helped to keep me sane. Vanja Klepac, Lara Gulmann, Jen Hammock, Matt Sullivan, Sarah Marsh and Annette Frese made for a dynamic group to start the program off with and have provided much support and friendship over the past five years. Thank you to Robert Weir for support, patience and friendship over the years.

Adam Frankel and Suzanne Yin made numerous contributions to this thesis while we were marooned in a mansion on a beach in California.

Special thanks to M.J. Tucci and J. Fenwick for help with all the ins and outs of grants and funding at WHOI.

Finally, thank you to my committee members: Christopher W. Clark, Darlene R. Ketten, Scott Kraus, and John Leonard. You have all taught me so much and it has been a pleasure to get to know each one of you better.

Support for S. Parks was provided by a Department of Defense National Defense Science and Engineering Graduate Fellowship, a Fye Teaching Fellowship, the Budweiser National Fish \& Wildlife Foundation Conservation Scholarship, Massachusetts Environmental Trust, Grant \# SC ENV 10009180177, Northeast Consortium (Univ. of NH) Agreement \# 02-563, Agreement \# 02-560 and 03-675, National Fish and Wildlife Whale Conservation Fund, Grant \#1999-0110-0008, The Reinhart Coastal Research and the Packard Foundation and the Woods Hole Oceanographic Education Office. 


\section{Table of Contents}

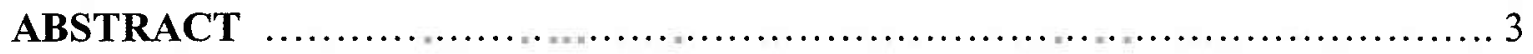

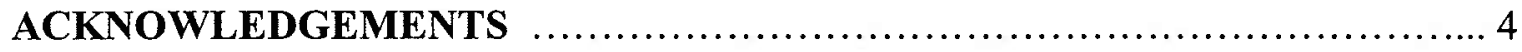

TABLE OF CONTENTS …........................................................... 7

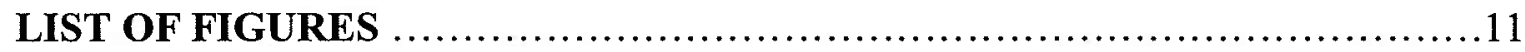

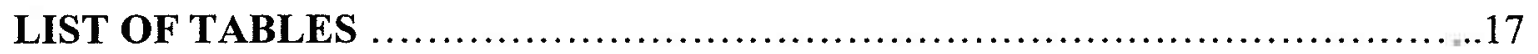

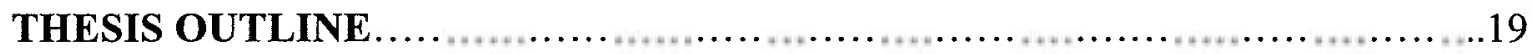

CHAPTER 1: SOCIAL INTERACTIONS IN BALEEN WHALES

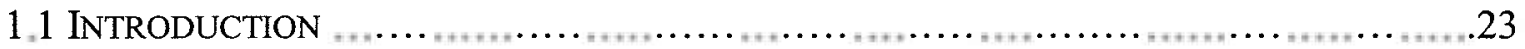

1.1.1 Social interactions in baleen whales on the breeding grounds ..............24

1.1.2 Male advertisement.............................................. 26

1.1.3 Male-Male competition............................................ 28

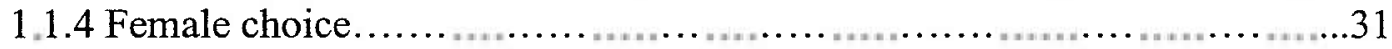

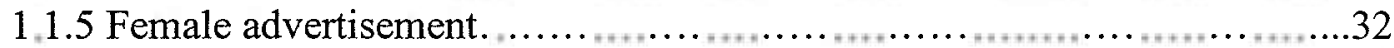

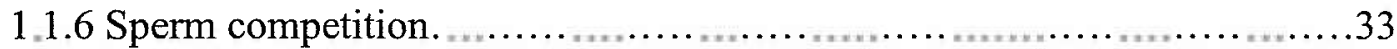

1.1.7 Social behavior on the feeding grounds.............................. 35

1.1.8 The use of sound for communication in the marine environment...........36

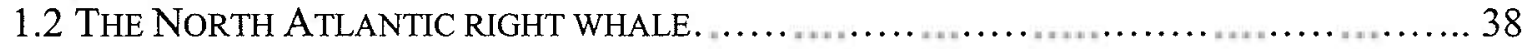

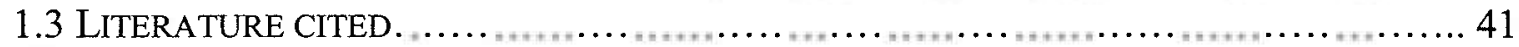

CHAPTER 2: SOCIAL BEHAVIOR OF NORTH ATLANTIC RIGHT WHALES (Eubalaena glacialis) IN SURFACE ACTIVE GROUPS

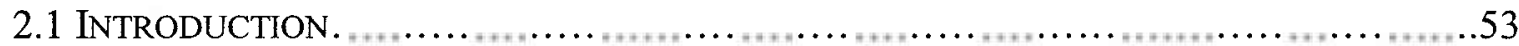

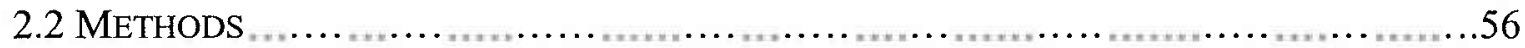

2.2.1 Sighting Catalog Database.............................................. 56

2.2.2 Corrections for occurrence........................................... 57

2.2.3 Analyses of catalog data............................................ 58

2.2 .4 Occurrence of SAGS ....................................................... 59

2.2.5 SAGs related to calf output............................................. 60

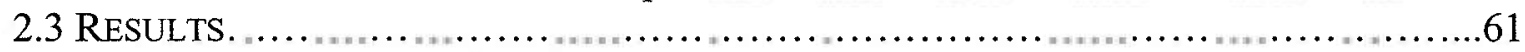

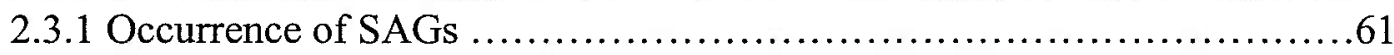

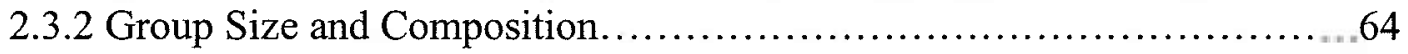

2.3.3 Individual Involvement in SAGs...................................... 69

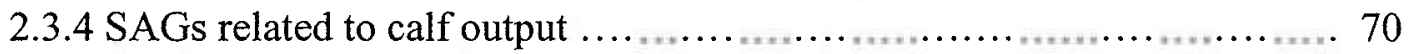

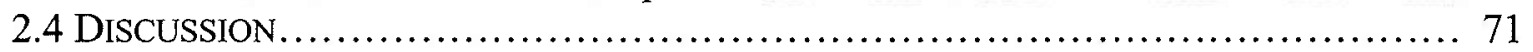

2.4.1 Occurrence of SAGs ......................................... 72

2.4.2 Group Size and Composition............................................. 74

2.4.3 Individual Involvement in SAGs.................................... 77

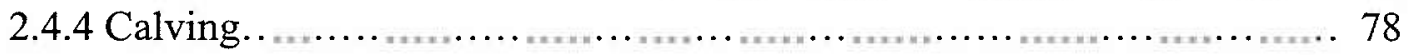


2.4.5 Conclusions and recommendations for further research.................. 78

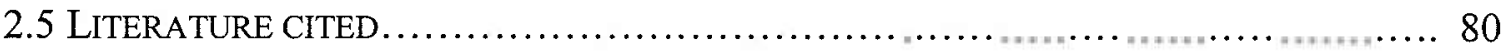

\section{CHAPTER 3: ACOUSTIC ACTIVITY OF NORTH ATLANTIC RIGHT WHALES (Eubalaena glacialis) IN SURFACE ACTIVE GROUPS}

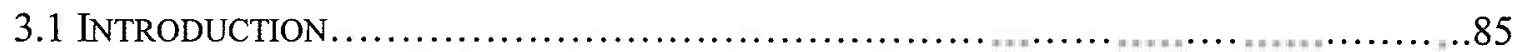

3.1.1 Right whale acoustics. .............................................. 86

3.1.2 The right whale repertoire: the classification problem................. 87

3.1.3 Sound production related to behavior.................................. 93

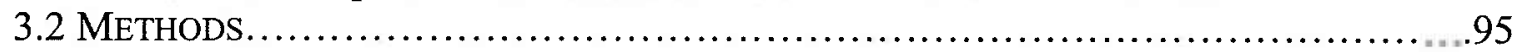

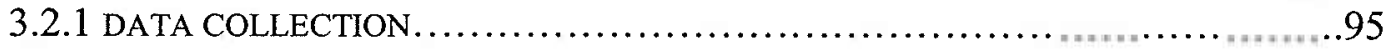

Sampling protocol...................................................95

Recording Equipment...............................................

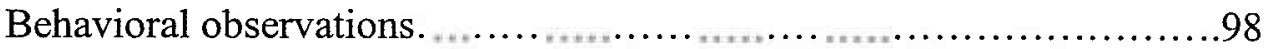

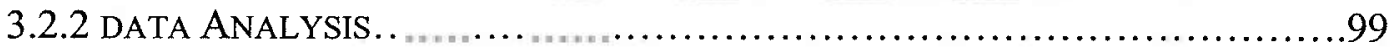

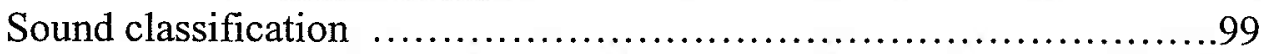

Call timing............................................................ 101

Individual sound production..........................................102

Source Level............................................................ 103

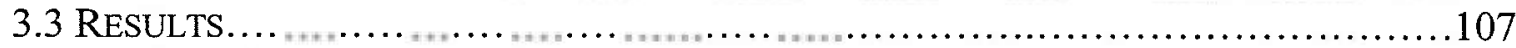

3.3.1 Surface Active Group Recordings...............................107

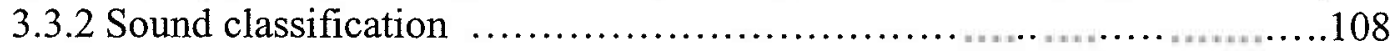

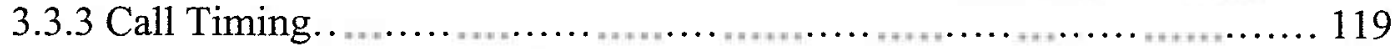

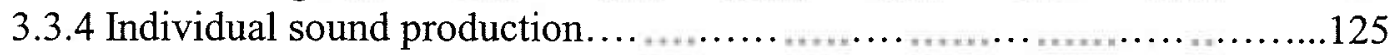

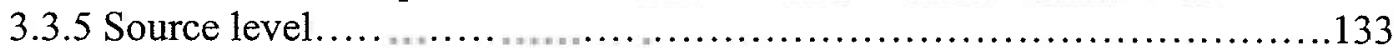

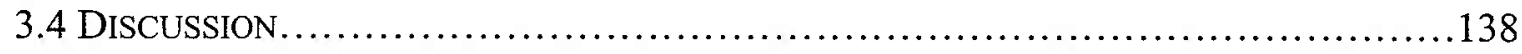

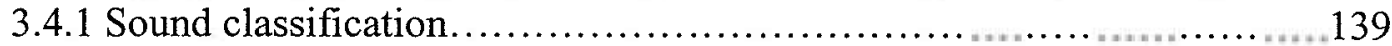

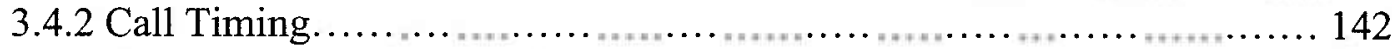

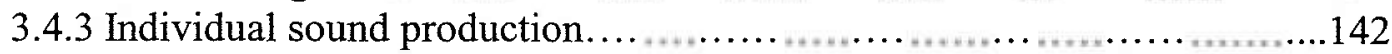

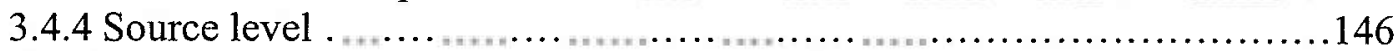

3.4.5 Possible improvements to be considered for future studies...............147

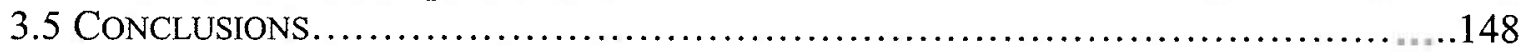

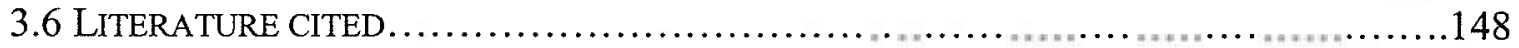

CHAPTER 4: PLAYBACK EXPERIMENTS TO NORTH ATLANTIC RIGHT WHALES IN THE BAY OF FUNDY

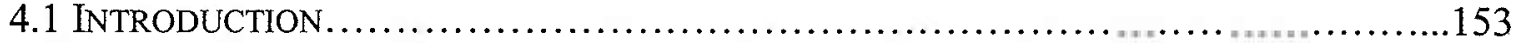

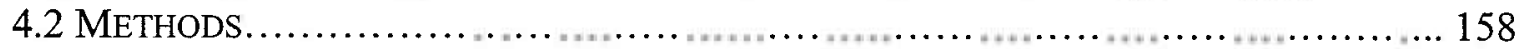

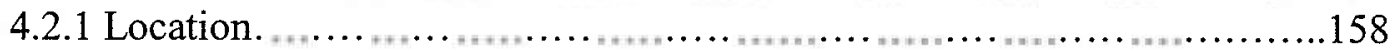

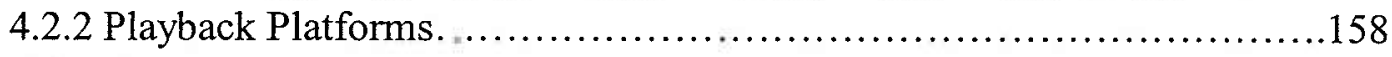

4.2.3 Stimuli........................................................ 158

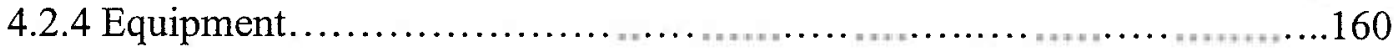


4.2.5 Projector source level................................................ 160

4.2.6 Playback protocol...................................................... 161

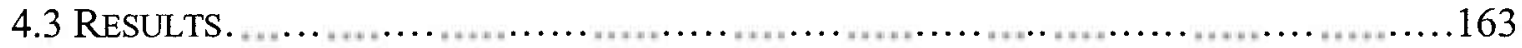

4.3.1 Response to the playbacks.......................................... 163

4.3.2 Differential response between sexes................................. 168

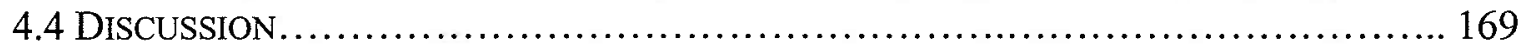

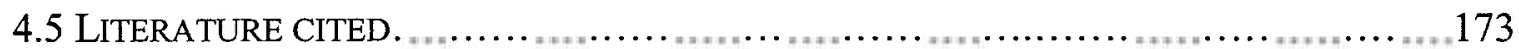

CHAPTER 5: HEARING IN THE NORTH ATLANTIC RIGHT WHALE: ANATOMICAL PREDICTIONS

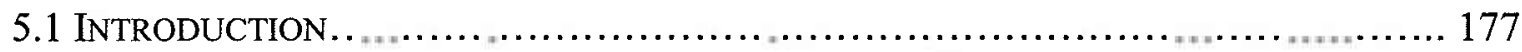

5.1.1. Hearing studies on other animals- A brief history...................... 177

5.1.2. Anatomical correlates with hearing sensitivity...........................178

5.1.3.Hearing studies in cetaceans......................................... 180

5.1.4. Anatomical modeling for marine mammal hearing......................181

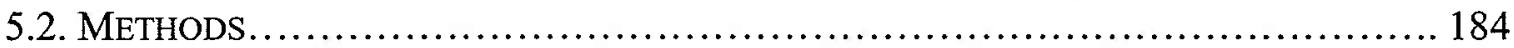

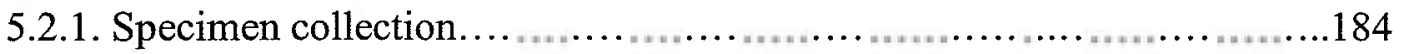

5.2.2. Computerized Tomography (CT) ....................................184

Number of turns in each cochlea.................................... 185

Cochlear length estimation.................................... 187

Ground truthing cochlea length calculations......................... 189

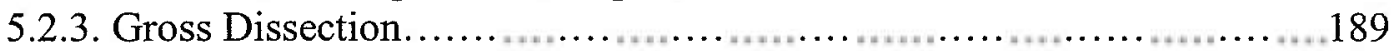

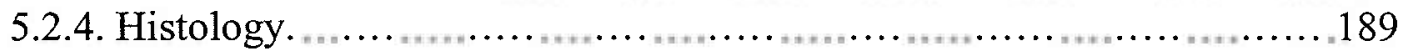

Basilar membrane measurements.................................190

Ganglion cell counts.........................................191

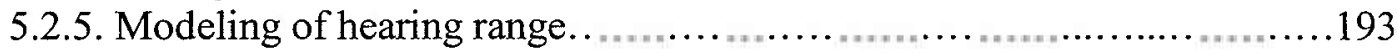

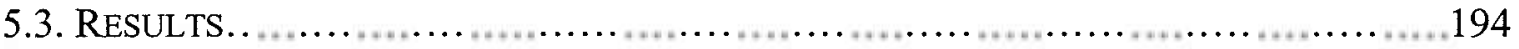

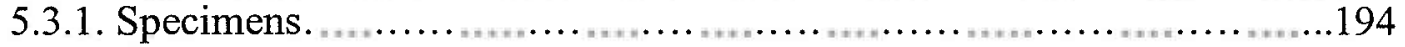

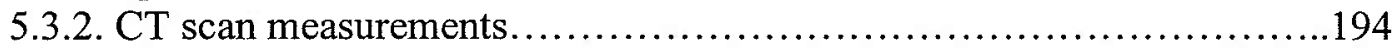

5.3.3. Cochlear length.................................................. 196

Reconstruction of cochlea..................................... 197

5.3.4. Body size versus cochlear length........................................197

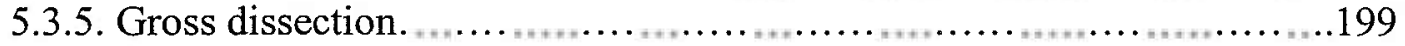

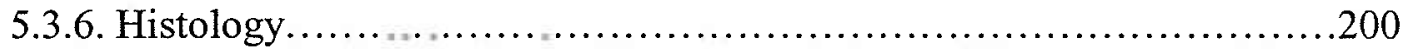

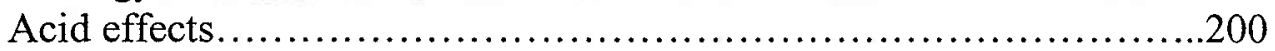

5.3.7. Basilar membrane measurements and ganglion cell counts.............202

5.3.8. Frequency range for right whale hearing................................206

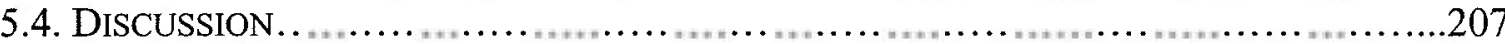

5.4.1. Specimen collection and preservation.............................207

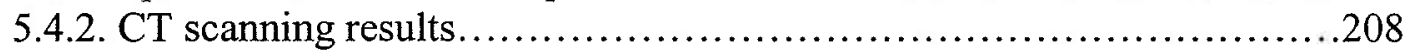

5.4.3. Cochlear length measurements........................................209

5.4.4. Cochlear length related to body size......................................209

5.4.5. Gross dissection. .................................................... 210 


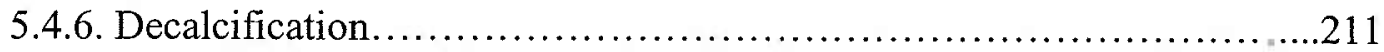

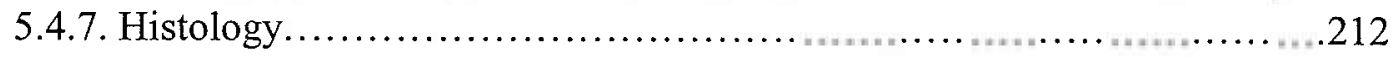

5.4.8. Basilar membrane measurements and ganglion cell counts...............213

5.4.9. Hearing range for the right whale.................................. 214

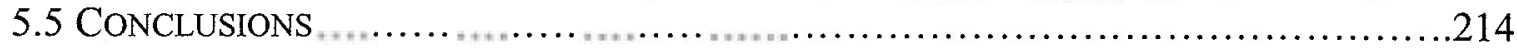

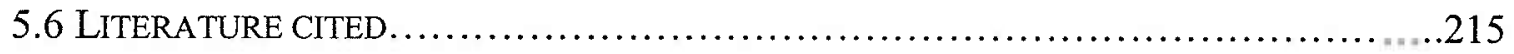

\section{CHAPTER 6: SUMMARY AND CONCLUSIONS}

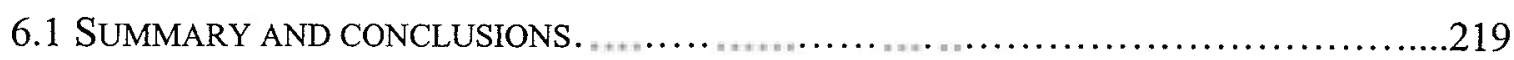

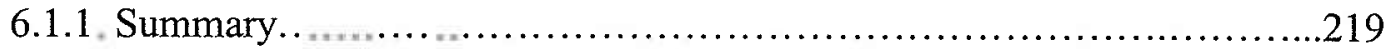

6.1.2. Chapter 1: Social interactions in baleen whales.........................219

6.1.3 Chapter 2: Social behavior in surface active groups.......................220

6.1.4. Chapter 3: Acoustic activity in surface active groups ....................226

6.1.5. Chapter 4: Playback in the Bay of Fundy................................231

6.1.6. Chapter 5: Hearing in the North Atlantic right whale....................234

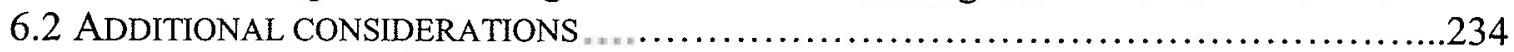

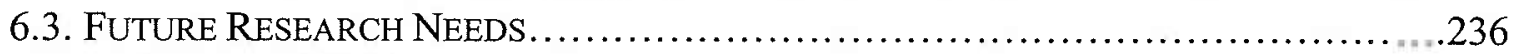

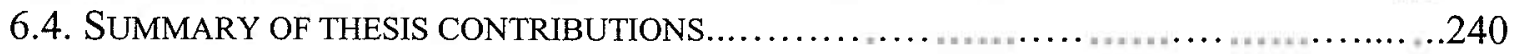

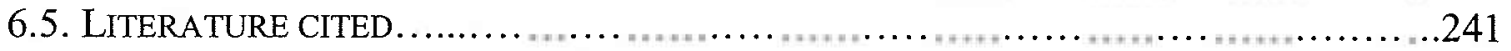

APPENDIX 1. SAG SIGHTING HISTORY FOR FOUR NORTH ATLANTIC

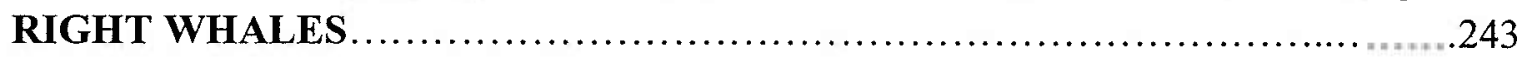

APPENDIX 2. GUNSHOT SOUND PRODUCTION BY THREE ADULT MALE NORTH ATLANTIC RIGHT WHALES IN THE BAY OF FUNDY

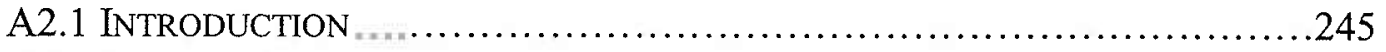

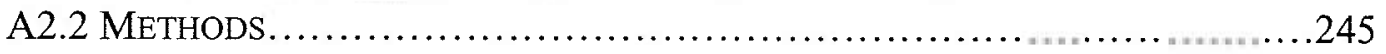

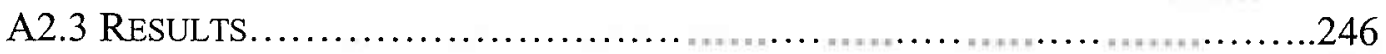

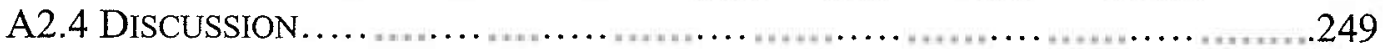

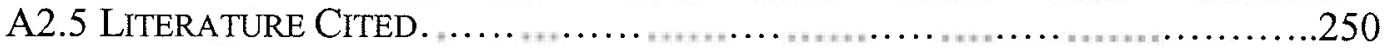

\section{APPENDIX 3. USE OF A DIGITAL ACOUSTIC TAG TO DOCUMENT RESPONSE OF THE NORTH ATLANTIC RIGHT WHALE TO SURFACE ACTIVE GROUPS}

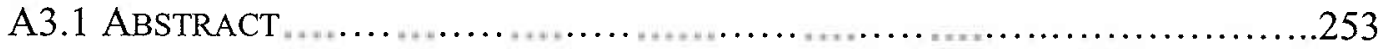

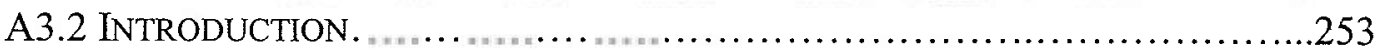

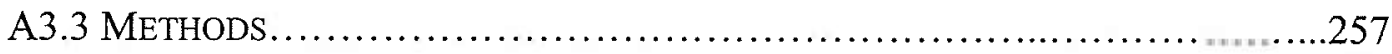

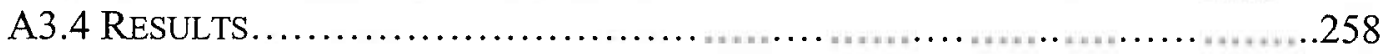

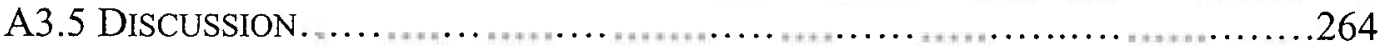

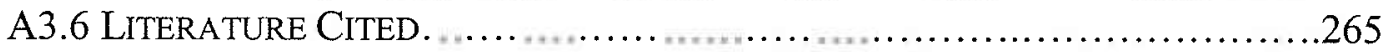

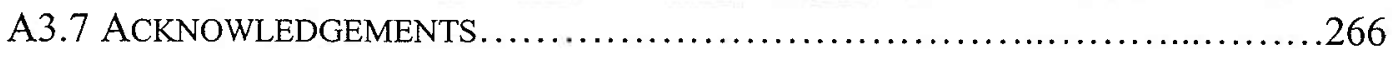




\section{List of Figures}

Figure 2.1. Number of SAGs sighted per year from 1992-2001 ........................62

Figure 2.2. Average number of SAGs seen per month between 1992 and 2001 ...........63

Figure 2.3. Average of total number of SAGs seen each month in each year divided by the total number of individual whales sighted in that given month for all years between 1992 and 2001.

Figure 2.4. Average values for the percentage of sightings made each month of right whales in SAGS from 1992 to 2001. .64

Figure 2.5. The proportion of 677 identified SAGs that fell into each of the seven SAG categories. .65

Figure 2.6. Plot showing the distribution of potentially reproductive SAGs as a percentage of all SAGs sighted in given month averaged over ten years..................66

Figure 2.7. Plot showing the distribution of non-reproductive SAGs as a percentage of all SAGs sighted in given month averaged over ten years. .67

Figure 2.8 The proportion of the SAGs of each type in a given region...... .68

Figure 2.9. Percentage of sightings in SAG for individual a) female and b) male right whales.

Figure 2.10. Percentage of known age whales first sighted in SAGs between 0-10 years. $0=$ year of birth.

Figure 2.11. A plot of the total number of SAGs sighted in one year and the number of calves born at the end of that year................................................... 71

Figure 3.1. Position of the vessel during array recording .............................97

Figure 3.2. An example of a Scream call showing the $2-2.2 \mathrm{kHz}$ frequency band used for narrow band source level calculations.............................................106

Figure 3.3. Results from cluster analysis of Z-transformed acoustat measurements for a

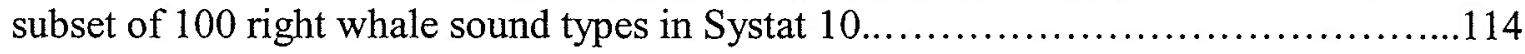

Figure 3.4. Results from PCA analysis showing the first two factors which preserve 38.6 percent of the variability. 116 
Figure 3.5. The same PCA results, showing the first two factors of the analysis with spectrograms for selected calls placed to help visualize the distribution of sounds.....116

Figure 3.6. Classification Tree produced in Systat 10, using minimum split index value of 0.001 for all 2992 sounds.

Figure 3.7. Classification tree of a subset of call types.

Figure 3.8. Average percentage of total calls produced of each class of calls by group composition.

Figure 3.9. Figure illustrating distribution of successive inter-call intervals between Screams and Gunshot from SAG 40 on Table 3.2.

Figure 3.10. Plots of counts of (a) inter-call intervals for Screams and for (b) intergunshot intervals.

Figure 3.11. A) Log survivorship plot of intercall interval between Screams. Dashed lines indicate the approximate slope for two parts of the curve. The black solid line indicates the bout criterion interval value determined from the intersection of the two slope lines. B) Log survivorship plot of inter-Gunshot interval between Gunshots.

Figure 3.12. The graphs from Fig.3.9 illustrating distribution of successive inter-call intervals between Screams and Gunshot from SAG 40 on Table 3.2 .....................123

Figure 3.13. Proportion of rates of calls per minute for A) Screams and B) Gunshots...124

Figure 3.14. Correlation of group size versus A) the maximum Gunshot sound per minute rate The correlation value was $r=.508$. B) Maximum Scream call per minute rate for each SAG r $=.208$.

Figure 3.15. Distribution of differences in bearing to sounds from the array and observed bearings to sources.

Figure 3.16. Difference in degrees between the array bearing to the sound source of a) Screams and b) Gunshots and the visual bearing to the center of the SAG being recorded

Figure 3.17. Difference in degrees between the array bearing to the sound source of a) Upcalls and b)Warbles and the visual bearing to individual whales outside of the SAG. 
Figure 3,18. Classification and Regression tree of Scream calls sorted by the age of the focal female in the group. The tree was constructed in Systat 10 with a minimum split index value of 0.010 .

Figure 3.19. Canonical scores plot resulting from discriminant analysis of Screams produced by 15 different individuals. Some individuals were recorded in more than one

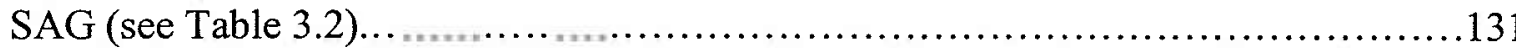

Figure 3.20. CART analysis of Screams by different individual focal females. The tree was constructed in Systat 10 with a minimum split index value of $0.010 \ldots \ldots \ldots \ldots \ldots \ldots . . .133$

Figure 3.21. a) Distribution of calculated broadband SL for Scream calls b) Measured broadband noise levels c) Calculated 2000-2200 Hz SL for Scream calls d) Measured

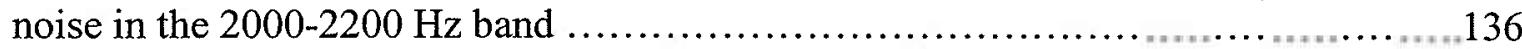

Figure 3.22. An example of a SAG recording made with another research vessel and a SAG approximately $200 \mathrm{~m}$ away.

Figure 3.23. Plot showing the measured noise levels and the calculated source levels for the right whale Screams both broadband and in the $2000-2200 \mathrm{~Hz}$ band. These values were used to roughly estimate the range that right whales may be able to detect these signals. .

Figure 3.24. Plots showing the signal-to-noise (SNR) for right whale Screams versus the ambient noise level measured in the same frequency band a) Distribution of the broadband SNR b) Distribution of the 2000-2200 Hz SNR. Note the higher SNR for the 2000-2200 $\mathrm{Hz}$ band.

Figure 3.25. Plot showing the calculated SL for the Scream calls versus the measured NL 1 second before the call. There was no apparent correlation between SL and NL in the broadband measurements, but indication of a slight correlation between SL and NL in the $2000-2200 \mathrm{~Hz}$ band. 138

Figure 4.1a-d. Four spectrograms showing examples of North Atlantic right whale surface active group Scream calls.

Figure 4.2. Bay of Fundy locations of playbacks between 1999 and 2001

Figure 4.3. Sighting positions and tracks of individual whales $(n=8)$ seen within $2000 \mathrm{~m}$ of the playback vessel (center of the plot) during the playback trial on 31 August 2000 
Figure 4.4. A) Approach response vs. no approach response observed in each trial for each of the seven stimuli presented for playback. There was no significant difference between any of the SAG playback stimuli. B) Approach response vs. no approach response to SAG $(n=31)$ and Gunshot $(n=5)$ playbacks (NAGS).

Figure 5.1. 3-D reconstruction of the entire temporal bone complex from specimen EG5. The reconstruction is from CT scans $.5 \mathrm{~mm}$ sections. The tympanic and periotic are labeled in each image. A) Medial view B) Anterior view C) Lateral view.

Figure 5.2. 3-D reconstruction of the cochlea from specimen EG 6 A) lateral view showing the rise of the right whale cochlea, the $8^{\text {th }}$ and the facial nerve B) Top view of the cochlear spiral illustrating the number of turns in the right whale cochlea...........186

Figure 5.3. 3-D reconstruction of the ossicular chain from a right whale. The figure shows the in situ orientation, the angle between ossicles, as well as the position of the ossicles relative to the cochlea. A cross section through the cochlea can be seen on the right side of the figure.

Figure 5.4. Cross section through a right whale cochlea for CT measurements. A) Schematic showing the orientation of the cross section B) An example of the resulting 2D CT image from the cross section.

Figure 5.5. Illustration of the measurements made from 2D CT cross sections from right whale specimens with 2.5 turns. .188

Figure 5.6. Closer view of basilar membrane from EG9 marking points for measurement of the width of the membrane. The thickness of the membrane was measured in the center of the membrane.

Figure 5.7. Ganglion cells from the basal turn of EG4. The ganglion cells in all ears showed evidence of cell loss, and all ganglion cells had a condensed appearance. The nerve fibers leading to the hair cells and the innter osseous spiral lamina are also labeled.

Figure 5.8. Images of reconstructed basilar membrane from EG4 with ganglion cell counts from the slide measurements.

Figure 5.9. A) Body length (measured from snout to notch of flukes) versus calculated cochlea length for all adult animals. B) Body length versus cochlea length for neonates, calves and young juveniles. The r-value is included in each plot..

Figure 5.10. Images from dissection of EG11 temporal bones. A) The medial view of the right ear. The tympanic and periotic bones are labeled. The $8^{\text {th }}$ nerve canal, round 
window and bony flange are also labeled. B) The lateral view of the same ear. The tympanic, periotic and flange bones are again labeled to aid in orientation.

Figure 5.11. Ossicles taken from EG 20 ear during gross dissection illustrating the shape and size of right whale ossicles. 200

Figure 5.12. Images from histology slide preparations from specimen EG 9. A) A midmodiolar cross section showing the layout of a right whale cochlea. The basal and apical turns are labeled. The VIIth nerve is also labeled. The basal turn is marked by a white circle. B) The basal turn from A) under higher magnification.........................200

Figure 5.13. Impact of acid decalcification on mysticete whale ear bones. .201

Figure 5.14. Insertion of the stapes into the oval widow and residual amniotic fluid in the middle ear cavity from $\mathrm{EG} 4$. .202

Figure 5.15. Reconstructions of basilar membranes and ganglion cell counts for each ear.

Figure 6.1. Relationship among mysticete species based on total evidence molecular data (Hasegawa et al. 1997). The darker branches indicate species with documented observations of SAG-like behaviors....................................................235

Figure A2.1. Figure AII.2. Spectrogram of a sequence of Gunshot sounds recorded on $8 / 11 / 00$ from an adult male right whale (catalog number 1424).

Figure A2.2. Example of Scream-like call production recorded on 8/11/00 from an adult male right whale (catalog number 1424). Note the first call in the sequence is an Upcall

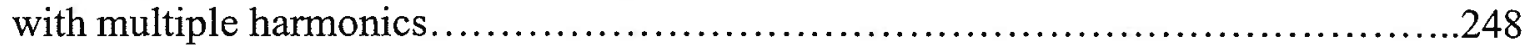

Figure A2.3. A typical surfacing sequence seen from adult male \#1307 on 9/6/00. The whale would 1 )come to the surface, 3 ) then roll on his side and make flipper slaps, 4) make head lifts before his dive and 5) raise his flukes high out of the water before the dive. Then he would repeat the sequence on the next surfacing. The headlift high fluke raising behavior were observed in all three males....

Figure A3.1. Figure from Kraus \& Hatch 2001 showing the typical formation of a surface active group.

Figure A3.2. Spectrogram of a sequence of five Scream calls recorded from a Surface Active group in the Bay of Fundy.........................................................256

Figure A3.3. A) A photograph of the DTAG before attachment and B) the DTAG on a whale. 
Figure A3.4. Approach vs. no response during SAG playback trials in 1999 and $2000 \ldots$

Figure A3.5. A plot showing response of a tagged right whale to a SAG playback. The depth, pitch, angular fluke stroke rate and acoustic record are included in the figure...260

Figure A3.6. A plot showing surface observations of a right whale during a SAG

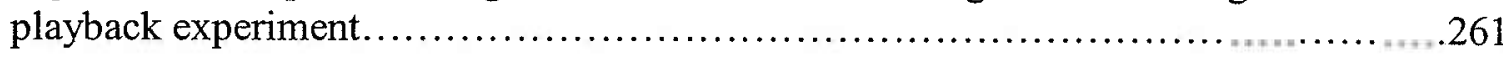

Figure A3.7. Figure showing observed depth and heading of the same whale from figure A3.6 using the DTAG data..

Figure A3.8. A combined pseudo-plot of the whale underwater during the playback trial, indicating that the whale turned toward the playback vessel while on a dive. .263

Figure A3.9. Pseudoplot of the track of a young male approaching an actual surface active group. The stars indicate the time at which Scream calls were recorded on the DTAG...... 


\section{List of Tables}

Table 2.1. SAG categories based on group composition..............................58

Table 2.2. A list of data collected for each SAG sighting record........................59

Table 2.3. A list of data collected for each individual related to SAG participation.......60

Table 2.4. The total number of SAGS sighted by month in each of the ten years..........62

Table 2.5. Correlation values for the number of SAGs versus the number of calves born the next year, and two years later..........................................................71

Table 3.1. Table from Clark (1983) showing the eight sound types found in Southern

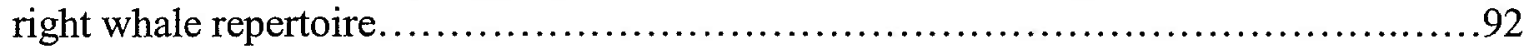

Table 3. 2. Table of all SAG recordings made between 1999-2002 in the Bay of Fundy

Table 3.3. Call types recorded in SAGs in the Bay of Fundy. Center frequency and duration were measured for 40 of each call type.

Table 3.4. Descriptive statistics for inter-call and inter-Gunshot intervals both within bouts defined by the measured bout criterion interval and between bouts of sound production.

Table 3.5. A table of analysis of video data from SAG observations listing the total number of Screams and Gunshots recorded on the video, the total number of breaths observed from the focal female, and the total number of Screams or Gunshots produced simultaneously with a breath from the focal female................................129

Table 3.6. The jackknifed classification matrix resulting from discriminant analysis between adult and juvenile focal females using all ACOUSTAT variables..............131

Table 3.7. The jackknifed classification matrix resulting from discriminant analysis for all focal females for which the identity was known...................................132

Table 3.8. Calculated SL for 3 call types and noise all measured as $\mathrm{dB}$ re $1 \mu \mathrm{Pa}$ broadband and from $2000-2200 \mathrm{~Hz} . \mathrm{N}=$ number of measurements; SL= calculated source level. Measurements for the Gunshot sound are broadband measurements from $0-20 \mathrm{kHz}$ and are also reported as $\mathrm{dB}$ re $1 \mu \mathrm{Pa}$. 
Table 3.9. The measured noise levels from the Bay of Fundy in summer of 2002. The noise was measured in the same frequency band.

Table 4.1. The date, stimulus type, level of response, number of whales seen from the playback vessel during a trial, the number of whales that approached the playback vessel during a trial, the age and sex of whales that approached the playback vessel and age and sex of identified whales that did not approach the playback vessel are given in the table.

Table 4.2. Summary of the age and sex of whales approaching and not approaching the playback vessel during A) NARW-SAG B) SARW-SAG playbacks. Adults are $>8 y$ r....

Table 5.1. A list of all specimens measured for this and whether the specimen was analyzed using CT or further processed for histology.

Table 5.2. Measurements taken from the CT scans for all specimens. The radii $(1 / 2 \pi$, $3 / 2 \pi, 5 / 2 \pi, 7 / 2 \pi$ and $9 / 2 \pi$ for $2.4-2.5$ turns, $0, \pi, 2 \pi, 3 \pi$, and $4 \pi$ for 2.25 turns), axial height and basal diameter are reported in mm. The number of turns are counts made from $3-\mathrm{D}$ reconstructions of the cochlea for each specimen. The axial pitch $=$ axial height/number of turns and the basal ratio = axial height/basal diameter.

Table 5.3. Measurements used for the calculation of length of the cochlear canal.

Table 5.4. Comparison between the calculated cochlea length from $\mathrm{CT}$ radii measurements and the length of the cochlea measured directly from 3-D reconstructions

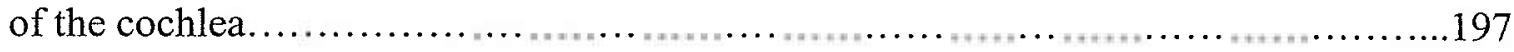

Table 5.5. Calculated ganglion cell density per mm of the basilar membrane.........203

Table. 5.6. The thickness/width ratio of the basilar membrane measurements at different $\%$ of membrane length (apex $=0$ ). The predicted frequency range corresponding to these measurements is listed in $\mathrm{kHz}$.

Table A2.1. Table showing the date, time, location, duration of observations and total

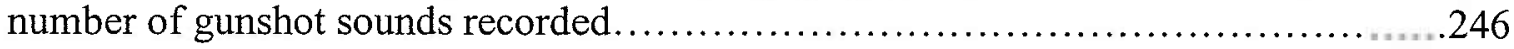

Table A2.2. Number of gunshots, number of breaths visible on video, and the number of overlaps of breathing with gunshot sound production.

Table A3.1. The date and duration of attachment of tag to right whales in the Bay of Fundy in 2000. The playback to the whale was either of SAG sounds (W) or vessel noise (V). 


\section{Thesis OUTLINE}

Surface active groups (SAGs) are potentially the most intriguing and least understood interaction in North Atlantic right whales (Eubalaena glacialis). The goal of this thesis is to investigate the use of sound for communication in these SAGs. This research addresses issues of social interactions, communication and perception in an endangered baleen whale species. The thesis is divided into six chapters. A brief summary of the hypotheses considered in each chapter is provided here.

\section{CHAPTER 1: SOCIAL INTERACTIONS IN BALEEN WHALES}

This chapter provides a review of the current literature on social behavior in coastal baleen whale species. Evidence that social interactions may play a significant role in their biology is highlighted.

\section{Hypothesis:}

1. Long-term social bonds between individuals are potentially an important aspect of baleen whale biology.

\section{Chapter 2: Social behavior of North Atlantic Right whales (Eubalaena glacialis) IN SURFACE ACTIVE GROUPS}

This chapter includes analyses of 1992-2001 right whale sighting records from the Right Whale Consortium Database to determine occurrence, distribution, and composition of SAGs in the Western North Atlantic. Further analyses were made for sighting records of individuals to determine if there are age or sex differences in SAG participation.

\section{Hypotheses:}

1. The number of SAGs observed through out the year will vary independent of annual survey effort reflecting season trends in behavior.

2. The reproductive nature of SAGs will be reflected in the group composition. 
3. Males will be sighted in SAGs more frequently than females.

4. If SAGs involve fertilization, the number of SAGs observed will be correlated to the number of calves born 11-13 months later.

5. Group composition of SAGs will differ at different times of the year.

\section{Chapter 3: ACOUSTIC ACTIVITY Of North Atlantic Right Whales (Eubalaena glacialis) IN SURFACE ACTIVE GROUPS}

The goal of this chapter is to describe the sounds produced by right whales in SAGs in the Bay of Fundy. The analyses include call timing, differences in sound production by different age and sex classes, and source level.

\section{Hypotheses:}

1. Particular types of sounds will be produced in all SAGs.

2. Sound production will vary with group composition reflecting differences in function.

3. Call rate is correlated to the group size.

4. The number of gunshots produced in a SAG will be correlated to the number of males present in the group

5. The focal animal produces the Screams in SAGs.

6. Males produced the Gunshot sounds in SAGs.

7. The focal animal does not produce gunshot sounds in SAGs.

8. Individual variation in Scream calls allow for individual recognition.

9. Source level for right whale sounds will vary with ambient noise levels.

\section{Chapter 4: PlaybaCK eXPeriments to NORTh ATLANTIC RIGHT WHALES IN THE} BAY OF FUNDY

This chapter presents results from SAG sound playback experiments. The experiment involved three stimuli; 1) Scream calls recorded from North Atlantic right 
whales 2) Scream calls recorded from South Atlantic right whales or 3) Gunshot sounds recorded from North Atlantic right whales. Differences in response to the three stimuli are discussed.

\section{Hypotheses:}

1. Playback of Screams from SAGs will attract right whales.

2. Playback of Gunshot sounds from SAGs will not attract right whales.

3. Scream calls from a SAG will attract significantly more males than females.

4. Playbacks of SAG sounds will attract more adults than juveniles.

5. Gunshot sounds may stimulate approach from mature males who may challenge the Gunshot playback by the producing more gunshots.

\section{CHAPTER 5: HEARING IN THE NORTH ATLANTIC Right WHALE: ANATOMICAL PREDICTIONS}

This chapter describes that anatomy of the right whale inner ear, with a focus on the dimensions of the cochlea and the basilar membrane. Measurements of the basilar membrane are used to estimate the frequency range of hearing for right whales.

\section{Hypotheses:}

1. The range of hearing sensitivities in the right whale will correspond to the range of frequencies produced in right whale vocalizations.

2. Anatomy of right whale ears will be similar to that of other baleen whale species.

\section{Chapter 6: Summary AND CONCLUSIONS}

This chapter summarizes the findings from the first five chapters. Specific results addressing the hypotheses listed here are included. Additional discussion of the role of SAGs within the framework of the right whale social structure and suggestions for future research are also included. 
Thesis outline 


\section{ChAPTER 1: Social INTERACTIONS IN BALEEN WHALES}

\subsection{INTRODUCTION}

The general consensus from current literature suggests that baleen whales do not form or maintain complex or long-term social bonds (Berta \& Sumich, 1999). The long migrations undertaken by most baleen whale species, coupled with their grazing method of feeding has led many to characterize them as herd animals (Berta \& Sumich, 1999). Strong social bonds between individuals are thought to be limited to the mother-calf pair bond in many species (Connor, 2000; Whitehead \& Mann, 2000). This contrasts with the very complex social interactions that have been described in other marine mammals species, particularly the odontocetes (Tyack, 1986). The discovery of strong social bonds relating to family groups (Bigg et al., 1990; Balcomb et al., 1982) mating coalitions in males (Herzing, 1996), or cooperative feeding (Würsig \& Würsig, 1980; Steltner et al., 1984; Würsig, 1986; Silber et al., 1990; Frost et al., 1992) in odontocetes may be a result of both natural history and intense long-term observation and experimental work with these species. Do baleen whales truly lack the strong social bonds that are common in the odontocetes or has a lack of appropriate data led to this assumption?

The best studied odontocete species typically consist of resident populations where large number of individuals can be identified and life history is known for different individuals (e.g. bottlenose dolphins Tursiops truncatus in Sarasota, FL (Scott et al., 1990), Tursiops aduncus in Shark Bay, Australia (Connor \& Smolker, 1985), and killer whales (Orcinus orca) (Bigg et al., 1990; Ford et al., 2000)). The huge migratory range of baleen whales makes them less amenable to these detailed types of studies. In addition, the large number of individuals in a single baleen whale population often makes individual recognition challenging. A few populations of baleen whale species have been subject to long-term study. Individual identification provides exceptional opportunities to study behavior related to social interactions in these populations. At least four populations of baleen whales have studies where individuals have been identified over a 
long period of time: humpback whales (Megaptera novaeangliae) feeding in the 1) North Pacific and the 2) North Atlantic and right whales 3) Eubalaena glacialis feeding in the Bay of Fundy and the 4) Eubalaena australis feeding in the Southern Ocean. Behavioral studies suggest complex social interactions in these four populations ranging from cooperative feeding in humpback whales (Baker, 1985; Jurasz \& Jurasz, 1979; D'Vincent et al., 1985), to surface active groups in right whales (Kraus \& Hatch, 2001).

There is evidence of long-term bonds between non-related individual whales in several species of baleen whales (e.g. humpback whales (Weinrich, 1991), right whales (Hamilton, 2002)) and the assumption of lack of interaction may result from the limitations of the data due to the difficulty of observing and tracking baleen whales at sea. Additionally, researchers often use close physical proximity as a measure of association in animals. This is a very limiting definition for baleen whales in particular that migrate thousands of miles and are potentially capable of communicating acoustically with one another over tens or hundreds of miles (Payne \& Webb, 1971). Baleen whale social interactions need not involve much, if any, close physical contact to be meaningful. It is possible that the general assumption that long-term social bonds are not important for baleen whales is a result of lack of the appropriate data, rather than a lack of long-term social bonds between baleen whales. More long-term studies of baleen whale populations are required to determine this.

\subsubsection{Social interactions in baleen whales on the breeding grounds}

For all baleen whales where a known calving and breeding ground exists, extensive data relate complex behavioral interactions believed to be part of competition and advertisement for mates. Suggestions have been made that baleen whale mating strategies run the gamut from male advertisement and female choice, male-male competition to female advertisement and sperm competition (Connor et al., 2000). While attempts have been made to describe these systems in terms of typical terrestrial mating systems (Clapham, 1996), life history traits and environmental dynamics of 
baleen whales may result in mating systems that are different from those seen in most terrestrial mammals. Baleen whales are thought to have long life spans ranging from an estimated 50 years in the small minke whale (Balaenoptera acutorostrata) (Evans \& Stirling, 2001) to over one hundred years in the bowhead whales (Balaena mysticetus) (George et al., 1999). This longevity leads to the possibility of long-term relationships developing between individuals that may encounter each other over many decades. It would be challenging to document interactions between individuals over such long time scales.

The 3-dimensional fluid aquatic environment makes it difficult to control resources. For example, it is not possible for baleen whales to defend food because they feed on patches of abundant, yet ephemeral, food sources. Males may advertise the presence of a food resource to attract females to an area (Croll et al., 2002) but the male may not be able to control the movements of the female if she does respond. In water, females have a 3-dimensional escape route to avoid unwanted copulations and the nearly opaque oceanic environment makes it possible for an animal to sneak away undetected. This gives females a distinct advantage in having almost unlimited escape routes to avoid unwanted mating or mate guarding by males. This suggests that coercion by males would rarely be effective in mating in baleen whales. It has been suggested that in gray whales (Eschrichtius robustus) and right whales, pairs of males cooperate to mate with a female by preventing her escape (Norris et al., 1983; Kraus \& Hatch, 2001). Some marine mammals, such as pinnipeds, mate on land. This makes it possible for males to guard or coerce females for mating. However, even in these mating systems, there is growing evidence that much of the mating must take place in the water as the males present on the beach are not gaining all the paternity of the pups (Wilmer et al., 1999).

Marine ecologists have attempted to explain observed whale reproductive behaviors in terms of well known mating systems described in terrestrial behavioral ecology. Suggestions have been made that baleen whale mating strategies span a wide range of mating strategies from male advertisement, male-male competition and female choice to female advertisement and sperm competition (Connor et al., 2000). In wide 
ranging pelagic species it is likely that female choice can drive mating systems as males have a limited ability to control either females or resources. The question of how female choice functions in whales is still unknown. Females may choose from males displaying in a type of lek as has been suggested for the humpback whale (Clapham, 1996). Females may incite competition between males resulting in copulation with the competitively superior male, as has been suggested for right whales (Kraus \& Hatch, 2001). Lack of data make it difficult to answer questions about the mating systems for most baleen whale species.

\subsubsection{Male advertisement}

Reproductive advertisement is a burden most frequently carried by the male of a species. This has resulted in a traditional view of reluctant females and ardent males (Darwin, 1871). Reproductive advertisement is a burden because the same signals that a male can use to attract a female (such as bright coloration or loud vocalizations) can also attract predators and parasites (Zahavi, 1975; Robert et al., 1992; Lehmann \& Heller, 1998; Warner \& Dill, 2000). Most terrestrial studies have focused on species where males advertise to attract females for mating by 1) displaying elaborate songs and vocalizations (e.g. European warblers (Acrocephalus schoenobaenus) (Catchpole, 1980) and the gray tree frog (Hyla versicolor) (Gerhardt \& Watson, 1995)), 2) displaying elaborate traits (e.g. horns in mountain sheep (Ovis canadensis) (Geist, 1971) or the long tails of the widowbirds (Euplectes sps.) (Andersson, 1982)), or 3) by an elaborate display of resources (the bower bird (Ptilinorhynchus violaceus) (Kevles, 1986)).

Males may also establish dominance hierarchies before females arrive, such as in herds or in lek displays (Emlen \& Oring, 1977). These mating systems often allow females to evaluate males and choose the best of " $n$-males". In many systems it is unlikely that a female can select the "best" male because of limitations from timing of fertility, energetic costs and predation risks involved with movement or a reduction in foraging efficiency while selecting mates. Theoretical studies of strategies for females in mate choice support the idea that the best-of- $n$-males strategy will yield the highest 
expected fitness for a mate (Janetos, 1980). It is possible that female whales are able to select from multiple displaying males when selecting a mate.

The song of the humpback whale is the best studied example of male reproductive advertisement of any baleen whale. Humpback whale song has been well described in the literature over the past thirty years (Payne \& McVay, 1971; Winn \& Winn, 1978; Hafner et al., 1979; Payne et al., 1983a; Guinee et al., 1983; Cato, 1991; Helweg et al., 1992; Frazer \& Mercado III, 2000). The songs are complex series of vocalizations performed by lone animals that last from $6-25$ minutes. All singers whose sex has been determined have been male (Helweg et al., 1992). The song is hierarchical in structure with short sound units that are combined into phrases. Phrases are combined to make themes which in turn are combined to makeup the songs (Payne \& McVay, 1971). The make up and order of themes are approximately the same among all individuals on a particular breeding ground. The songs evolve by alteration in the structure of the phrases throughout the season (Guinee et al., 1983). All whales in a local population appear to follow these changes. The song changes little between the end of one season and the start of the next season (Payne et al., 1983a). Song has been hypothesized to function as a:

1) sexual advertisement to females in the area, like bird song (Payne \& McVay, 1971)

2) territorial spacing mechanism for competing males (Winn \& Winn, 1978; Frankel, 1995)

3) mechanism to synchronize ovulation in females (Baker \& Herman, 1984)

4) navigational beacon to guide whales on the migration to the breeding sites (Winn \& Winn, 1978)

5) sonar to locate silent females swimming through the breeding ground (Frazer \& Mercado III, 2000)

Research efforts over the past 20 years have failed to conclusively show the ultimate functions of humpback whale song. Playback experiments indicate that the songs are generally not attractive. Whales exposed to playback of recorded song did not approach the playback vessel (Tyack, 1983). This was true for females who would be the most likely to respond if song was intended to attract females (Tyack, 1983). Additional work 
on the movements of females through a breeding ground full of singing males and her responses to individual singers may shed more light on the role that song may play in female choice.

Song has also been recorded in the bowhead whale, though their remote habitat in the Arctic has resulted in limited behavioral observations in the field. Bowhead songs have been reported during the spring migration and in the Bering Sea during the summer months (Würsig \& Clark, 1993). The songs usually consist of two themes that are composed of repeated phrases. The individual units in the songs differ from normal bowhead call types. Songs last for several minutes with individual song bouts lasting for several hours (Würsig \& Clark, 1993). The actual mating period of bowhead whales is unknown, but presumed sexual activity has been observed from January to early October (Koski et al., 1993). The calving period is from early April to early June (Koski et al., 1993 ) and the estimated duration of gestation is 13-14 months (Nerini et al., 1984; Koski et al., 1993). Therefore, it is possible that this song is primarily produced during the mating season, when whales are dispersed and moving allowing males to advertise to females as they are swimming by.

\subsubsection{Male-Male competition}

Direct male-male conflict over obtaining mates is common in mammalian mating systems. When males cannot control resources or advertise to attract the opposite sex, they may have to resort to competing directly for access to reproductive females. It has been documented that the more successful males in these competitions obtain more mating opportunities (Lott, 1979; Appleby, 1982; Hoelzel et al., 1999; Linklater et al., 1999). In baleen whales it is not possible for males to control the movements and activities of multiple females because females do not travel in distinct, close knit herds. Some evidence suggests that females may actively avoid one another (e.g. (Tyack, 1982)). Therefore, male-male competition in baleen whales would be expected for direct access to a single female. Conflicts between males for access to a female are seen in 
several baleen whales species (e.g. gray whales and right whales (Norris et al., 1983; Kraus \& Hatch, 2001)).

Arguably, the most violent male-male competition is seen in humpback whale competitive groups. Competitive groups on the mating ground are believed to represent intra-sexual competition between males for access to potentially estrous females (Clapham et al., 1992). Competitive groups consist of three or more adult whales involved in high levels of surface activity and aggression (Baker \& Herman, 1984). The central animal in the group, usually unresponsive to the approach of other individuals and typically female, is termed the Nuclear Animal (NA) (Tyack \& Whitehead, 1983). The whale consistently in close contact with the nuclear animal is termed the Principal Escort (PE). Those PEs for which the sex has been determined have been male. Other whales in the group are termed Secondary Escorts (SE), also typically male. SEs may occasionally replace the PE or leave and join the group (Tyack \& Whitehead, 1983). SEs frequently challenge the $\mathrm{PE}$, leading to aggressive interactions and occasionally violent physical contact. Sounds recorded from these groups include slap and rasping sounds thought to be associated with body contact between individuals (Tyack \& Whitehead, 1983).

Gray whales form large social groups at lagoon entrances in Baja California. These groups typically involve males competing to reach a stationary central female (Norris et al., 1983). The most common group composition is two males with a single female (Samaras, 1974). Some researchers have observed several of these smaller groups within $100 \mathrm{~m}$ of each other (Norris et al., 1983) and groups with up to 18 gray whales have been documented (Swartz, 1986). Males in these mating groups nudge each other but the interactions appear not to be as aggressive as those seen in humpback whales. These courtship groups have been observed on the feeding grounds, during migration, and on the calving grounds. All-male groups have also been observed in this species (Norris et al., 1983). Norris et al. (1983) hypothesized that gray whale social groups serve some social signaling function and suggested a neutral term "wuzzling" to describe these groups without reference to reproductive function. 
Social groups have been described for the bowhead whale (Richardson et al., 1995). Social activity in bowheads consists of groups of at least 2-3 whales that engage in high levels of surface activity including tail lofts, tail and flipper slaps, rolls, chases, caresses and sexual interactions. These groups often have a central animal belly-up in the middle of the group, which seems similar to the central animal in gray whale groups. In two out of three cases however, these central animals have been sexed as male, while the flanking animals are often males based on observable penises (Richardson et al., 1995). High rates of vocalizations have been recorded near these groups, representing all known types of bowhead calls described in Würsig and Clark (1993).

Right whales also engage in large social groups, and these groups have been described by various researchers as courtship groups in Southern Right whales (Donnelly, 1967; Payne, 1995) and surface active groups in North Atlantic right whales (Kraus \& Hatch, 2001). The groups range in size from two individuals up to 30 or more in a single group. In the North Atlantic the most common composition of these groups in the Bay of Fundy involves a single female surrounded by multiple males (Kraus \& Hatch, 2001). The female is called the focal animal and is typically at the center of the group. The groups have been seen in all known habitat areas of the North Atlantic right whale in most months of the year (Kraus \& Hatch, 2001).

One puzzling aspect of these social groups in baleen whales is that the composition of the groups is not always appropriate for a reproductive function. In all species except for the humpback, intromission has been observed in these groups on at least one occasion but all-male groups do not seem to be uncommon (e.g. humpbacks (Clapham et al., 1992), gray whales (Norris et al., 1983), bowheads, (Würsig \& Clark, 1993), and right whales (Kraus et al., 2001)). Males have been observed to put their penises into the genital slits of other males in at least two species (bowhead whales (Würsig \& Clark, 1993), right whales (H. Pettis, pers. comm.). It has been suggested that these all-male groups may function as practice behavior in males to improve performance when a female is actually involved (Kraus et al., 2001) or to establish dominance over other males as seen in other mammals (Yamane, 1999). 


\subsubsection{Female choice}

The potential reproductive output for females is usually much lower than that for males because of the large investment females typically make in gametes and/or parental care. Each reproductive event for a female is therefore both more valuable and more costly than it is for a male. As a result, the female is typically considered the "choosy" sex, rejecting most males who would attempt to copulate with her. While competition between males is often important in determining who will be successful in obtaining mates, the ultimate success often depends on females choosing to mate with the most dominant male. There is good evidence of females selecting mates in species where males provide material resources from food (Sakaluk, 1986), better territory (Wells, 1977; Yasukawa, 1981) or superior parental care (Norris, 1990). Females may prefer different males even when they only receive sperm from the males (Andersson, 1982; Gerhardt et al., 1995; Catchpole \& Slater, 1995). Increased fitness of offspring is predicted to be the only benefit to females in these instances of choice for "good genes". Female choice is believed to be a selective pressure for the development of elaborate displays and ornaments in males. Three hypotheses have been proposed to explain the directional selection pressure resulting from female choice in these instances. The first, honest advertisement, suggests that female preference for elaborate traits may be a result of the traits directly indicating males of higher quality (Hamilton \& Zuk, 1982). The second, Fisher's runaway selection hypothesis (Fisher, 1930), suggests that female choice for a particular male trait leads to excessive development of that trait after many generations of directional female choice (e.g. long tails in widow birds (Andersson, 1982)). In runaway selection, females are hypothesized to select males for extreme development of this trait which is preferred by females. If the trait is heritable, then sons will have an increased level of reproductive success in the next generation. The third, the handicap hypothesis (Zahavi, 1975), suggests that females prefer elaborate displays because they are a handicap to the male's ability to survive. If the male is able to survive in spite of his ornaments or displays, he must have other traits that help him survive. In 
the handicap hypothesis, female preference is for good survivorship ability in the male, rather than his ability to attract females.

Recent studies have shown that female preferences can lead to differential success of offspring when females are artificially mated to males they prefer versus males they avoid mating with (Doty \& Welch, 2001). More recently, studies have suggested that females choose to mate with genetically compatible males, adding another dimension to female choice (Zeh \& Zeh, 1997). The best male may not be the best mate if the genetic combination of the two parents results in reduced viability (Wilson et al., 1997). There is no direct evidence of female choice in any baleen whale species. Female choice has been inferred from the behavior of males on the breeding ground and the limited ability of males to control female movements.

\subsubsection{Female advertisement}

While sexual selection in most species is driven by male advertisement and female choice, in some animal mating systems female advertisement is used to attract mates when the female is receptive. These species with female advertisement are distinctly different than role-reversed species where the males are the limiting resource for females and females show traditional "male" behaviors in competition for mates (e.g. Jacanas (Jacana spinosa) (Jenni \& Collier, 1972) \& Seahorses (Hippocampus whitei) (Vincent \& Sadler, 1995)). Examples where females attract males for mating include the pheromone systems in moths (Acharya \& McNeil, 1998), pheromonal advertisement by female snakes (Kevles, 1986), hormonal advertisement in female elephants (Rasmussen \& Schulte, 1998), and the loud vocal mounting protestations in elephant seals (Cox \& LeBoeuf, 1977). These advertisements attract multiple males who then compete for access to the female, and therefore these systems can also be considered a form of female induced male-male competition. The female still mates with the 'victor' of a male agonistic encounter, but the female incites the competition. The female also attracts the males to her for competition rather than approaching males on leks or in herds where 
male-male hierarchies are established before the females arrive. In species where males do not provide any resources, females should act to fertilize their eggs with sperm bearing the best genes (Bradbury \& Vehrencamp, 1998). One strategy to assess male quality is to force potential mates to compete for access to fertilization. In species where the cost of searching for a mate may be very high, the ability of the female to attract multiple males and incite them to compete for access to her can increase the quality of offspring that the female is able to produce, while limiting the costs of finding and choosing a mate directly (Jennions \& Petrie, 2000).

There is only one known example in baleen whales that seems to fit this type of female advertisement, the courtship group or surface active group of the right whale species. The indirect observations of behavior related to sound production have led researchers to conclude that females produce calls that attract males. The males then compete with each other for sexual access to the female (Kraus \& Hatch 2001). Similar behaviors and groups observed in bowhead and gray whales merit further investigation to see what role the female plays in the formation and maintenance of these groups. It is possible that the role of female advertisement in baleen whales is more common than currently known.

\subsubsection{Sperm Competition}

Sperm competition is a cryptic form of reproductive competition. In sperm competition, the gametes from two or more males compete to fertilize the eggs. This competition is known to take three forms, displacement of previous inseminations, dilution of rival sperm, and plugging of the reproductive tract (Gomendio et al., 1998). The role of the female's anatomy and physiology in controlling sperm competition between males may be similar to the female's behavior of inciting competition between individual males. If sperm have to compete in the reproductive tract, the most competitively successful sperm would fertilize the eggs, increasing the possibility of having sons with competitively superior sperm (Knowlton \& Greenwell, 1984). In many 
species with sperm competition, the reproductive tract of the female is a hostile environment to sperm, often with low $\mathrm{pH}$ and many folds and blockages, which make access of the sperm to the egg more difficult (Eberhard, 1998). The reproductive anatomy of baleen whale females suggests that there may be some challenges for sperm to reach eggs for fertilization. Many species appear to have complex tissue arrangements at their cervix (Haldiman \& Tarpley, 1993), with the possibility that multiple cervixes must be crossed for successful fertilization. Additionally, evidence from terrestrial species suggests that genetic compatibility of the sperm and the egg may play an important role in successful fertilization (Kenagy \& Trombulak, 1986; Wilson et al., 1997; Zeh \& Zeh, 1997; Hoogland, 1998; Clark et al., 1999; Olsson \& Madsen, 2001). Much of this postcopulatory competition between sperm has been discovered recently in many species as a result of paternity analyses.

Males that compete via sperm competition typically have relatively larger testes and longer penises than males in species where females only copulate with one male (Harcourt et al., 1981; Kenagy \& Trombulak, 1986; Møller, 1989; Møller \& Ninni, 1998). Studies of the relative size of the testes and penis in baleen whales show that right whales, bowhead whales and gray whales all have larger testes size than would be predicted from their body weight. Blue (Balenoptera musculus), fin (Balaenoptera physalus), humpback, brydes (Balenoptera edeni), sei (Balaenoptera borealis), and minke whales all have testes that are the smaller than predicted based on body size. This suggests that sperm competition may play a role in reproduction for the right, bowhead and gray whales (Brownell \& Ralls, 1986). Similarities in the observed behavior in these three species support this theory, particularly in the large social groups with multiple males interacting with single females. Humpbacks are not predicted to have sperm competition. The higher rates of aggressive behavior between humpback males in competitive groups may represent direct competition of males for access to a female, rather than an attempt to compete via sperm competition (Clapham et al., 1992). 


\subsubsection{Social behavior on the feeding grounds}

Two of the best known social interactions on the feeding grounds in baleen whales are humpback cooperative feeding off the Alaskan coast (Jurasz \& Jurasz, 1979; D'Vincent et al., 1985) and right whale social groups in the Gulf of Maine (Kraus \& Hatch, 2001; Hamilton, 2002). In the case of the humpback, the direct benefit of cooperative feeding is in the successful capture of large balls of herring. In the case of right whales on the feeding grounds, the functional advantage of social groups, which often last several hours when the whales could be feeding, is less obvious.

The feeding groups of humpbacks provide the best evidence for the existence of long-term social bonds between baleen whales. For example, in southeast Alaska where humpbacks feed on swarming euphausiids, fluid, temporary associations are formed by humpbacks in the area, apparently regardless of age and sex (Baker \& Herman, 1985). In contrast, the humpbacks that feed on schooling fish further north near Glacier Bay, have shown long-term associations of the same groups of whales over several summers, feeding in a coordinated fashion on schools of fish. These groups tend to be composed of primarily adult females, though some adult males have been observed in the groups (Jurasz \& Jurasz, 1979).

It is not surprising that baleen whales interact on the breeding grounds and that most of these interactions can be explained as functioning in mate selection and reproduction. What is surprising is that many of these social interactions assumed to function for reproduction are seen in other parts the annual migratory cycle in many baleen whales. Behaviors that appear to be identical to those seen on the breeding grounds have been seen during migration (e.g. gray (Norris et al., 1983), humpback (Noad, 2002), North Atlantic right (Kraus \& Hatch, 2001) and bowhead whale social groups (Würsig \& Clark, 1993)) and on the feeding ground (North Atlantic right whale surface active groups (Kraus \& Hatch, 2001), humpback song (Mattila et al., 1987)) for most coastal baleen whale species. Most baleen whales have a distinct season for calving. 
It is expected that the mating in these species should also be seasonal. The occurrence of these interactions outside of the breeding season is puzzling.

What role can these social interactions play if they are not part of the mating season? Three hypotheses to explain the observed behavior include 1) baleen whales may have a physiological mechanism to uncouple the timing between fertilization and implantation of an embryo, such as delayed implantation, 2) baleen whales use social interactions for mate choice, allowing for fertilization to take place between individuals at a later date or, 3) social interactions play a significant role in baleen whale biology for functions other than reproduction.

The social surface active groups (SAGs) of North Atlantic right whales observed in the summer on the feeding grounds seem, from initial observation, to be analogous to the groups showing similar behavior on the calving/breeding grounds both in the southeastern U.S. (Kraus et al., 2001) and in southern right whales in the South Atlantic (Payne \& Dorsey, 1983; Donnelly, 1967). The common group composition in the Bay of Fundy (with a single adult female flanked by multiple adult males), coupled with the observation of intromission on several occasions suggests that these groups function for mating. Yet the timing and frequency of occurrence of the groups does not seem to be related to the production of calves. These groups have been observed on surveys in eleven months of the year and on both the calving and feeding grounds (See Chapter 2). Not only does participation mean an increase in energy expenditure from the interaction itself but the whales involved in SAGs are losing time from feeding. The long duration of the groups and high energetic costs indicate that these groups are an important part of a right whale's life, but the ultimate function of these groups remains a mystery.

\subsubsection{The use of sound for communication in the marine environment}

Communication is a key part of social interaction in any animal. It is particularly important in the marine environment where it would be difficult for individuals to randomly find one another in the vast ocean. Unlike the terrestrial environment, where 
visual cues can often be detected further than any other type of signal, in the ocean, sound is the most efficient and most highly utilized channel for long-range communication. Visibility is limited in the marine environment to a few hundred yards in the clearest surface waters. In much of the nutrient rich water that baleen whales swim to find their prey, visibility may be limited to less than ten meters. Clearly another form of communication will be important for whales to find one another, if for no other reason than to mate. Whales have taken advantage of the properties of sound in the ocean to communicate over long ranges using sound.

Sounds appear to function in all known social interactions between baleen whales. Humpback whales produce long elaborate songs as part of their male display (Payne \& McVay, 1971). Particular calls have been recorded that appear to coordinate the movements of humpbacks feeding on fish (Cerchio \& Dahlheim, 2001), and a variety of sounds have been recorded from the competitive groups (Tyack \& Whitehead, 1983; Silber, 1986). Bowhead whale song has not been associated with particular social interactions, but a variety of calls and slap sounds have been recorded from bowhead social groups (Würsig \& Clark, 1993). Gray whales produce distinct calls in their mating wuzzles (Norris et al., 1983). Right whales make calls that are associated with surface active groups (Clark, 1983; Kraus \& Hatch, 2001) and also produce sounds that seem to function as contact calls to bring individuals together (Clark \& Clark, 1980; Clark, 1983).

If sound mediates social interactions in baleen whales, it seems clear that using recordings of calls in conjunction with surface behavioral observations may aid researchers in distinguishing different social interactions. For example, in the North Atlantic right whale, group composition of SAGs include a single female with males, multiple females with males, all male groups and all female groups (Kraus \& Hatch 2001). It is unlikely that the functional role of each of these groups would be the same, but to human observers, the interactions look the same at the surface, and only individual identification can discriminate between types of groups.

It is possible that adding an acoustic component to these observations may make it possible to distinguish between different types of groups. The effective range over 
which baleen whales can use sound to communicate must also be considered. The source levels and frequency range of baleen whale calls indicate that whales may be able to communicate over long distances (Payne \& Webb, 1971). Whales that are many kilometers apart could be associated and keeping track of one another acoustically. This adds another dimension to consider for future research into baleen whale social interactions.

\subsection{The NORTH ATLANTIC RIGHT WHALE}

The North Atlantic right whale is a highly endangered species of baleen whale. Traditionally, right whale populations were divided between the Northern and Southern hemispheres, with the Northern right whale called Eubalaena glacialis and the Southern right whale called Eubalaena australis. These traditional species designations have come into question in recent years (Rice, 1998). Currently, three distinct populations of right whales are distinguished based on their distribution. Genetic analyses support reproductive isolation between the populations in the North Atlantic and the Southern Hemisphere (Malik et al., 2000). Further subdivisions have been proposed between the Pacific and Atlantic stocks into subspecies with the North Atlantic right whale as Eubalaena glacialis, the Southern right whale as Eubalaena australis, and the North Pacific right whale as Eubalaena japonica (Rosenbaum et al., 2000). All of these whales have the same external appearance; large baleen whales, $35-55 \mathrm{ft}$ in length with black skin, broad pectoral fins, broad tail, a flatted upper jaw and raised patches of skin on the head called callosities. Callosity patterns are individually distinctive allowing for individual recognition of animals in the population (Payne et al., 1983b; Kraus et al., 1986).

The common name of the right whale comes from its importance to the early whaling industry. The large amounts of blubber and long baleen plates made right whales the most profitable species for whalers to kill. The slow swimming speeds and buoyant properties of the blubber after death also made it one of the easier whales to kill. A combination of these factors led to early decimation of the stocks of these whales. For 
example, in the North Atlantic, tens of thousands were taken by both the Basque whalers before 1600 (Aguilar, 1986) and by American fisheries through the 1920s (Reeves \& Mitchell, 1986). All species of right whales are classified as endangered. The right whale was among one of the first species to be protected from large scale hunting in 1935. The Southern right whale has shown remarkable recovery. The southern hemisphere population is near 7500 . Current population growth rates are estimated at $6.8 \%$ (Best et al., 2001; Best, 1990). Illegal Soviet whaling in the mid-1900's may have significantly impacted the rate of recovery of the Southern right whale populations (Tormosov et al., 1998). The North Atlantic right whale and Pacific right whale populations have not shown the same recovery (Caswell et al., 1999; Brownell Jr. et al., 2001).

The small population size and endangered status of the North Atlantic right whale have resulted in extensive research over the past twenty years to monitor the status of the population and to help it recover (Hamilton \& Martin, 1999). The North Atlantic right whale (Eubalaena glacialis) population migrates along the east coast of the United States from northern waters in the Gulf of Maine in the spring and summer to the waters off the states of Florida and Georgia in the winter (Winn et al., 1986). Despite protection from whaling for the past 60 years, current estimates indicate that fewer than 300 North Atlantic right whales remain (Knowlton et al., 1994; IWC, 2001), making them one of the most endangered large whales in the world. Population growth in this population has dropped from a $2.5-5.3 \%$ increase in the 80 's, to a $2.4 \%$ decrease in the population by 1994 (Caswell et al., 1999). There appears to be a steady increase in the mortality rate (Knowlton \& Kraus, 2001) and calf production remains highly variable ranging from an all-time low in 2000 of one calf to an all-time high in 2001 with 31 calves being born. At this rate, extinction of the population is predicted within 200 years (Caswell et al., 1999). This apparent decline in reproductive success in this population is of serious concern, and information about their mating behavior is crucial to minimize human impact on reproduction.

The current interest in the reproductive output of the North Atlantic right whales has revealed many uncertainties in our current understanding of the mating behavior in 
this species. Several researchers have discussed mating behavior of right whales over the years (Donnelly, 1967; Clark, 1983; Kraus, 1991; Payne, 1995) often with an underlying assumption that observed mating behavior is always for reproduction. Papers discussing the unusually large testis and penis of the right whale (Brownell \& Ralls, 1986) hypothesized that sperm competition might characterize the right whale mating system. This hypothesis was further supported by the observations of large "courtship groups" with a single female surrounded by multiple males.

Several pieces of evidence argue against the simple interpretation of social groups functioning for fertilization. Right whales do form large mating groups in the calving grounds in the southern hemisphere but the females seen in those groups don't return the following year with a calf (Payne, 1995). Right whales in the northern hemisphere can be observed in SAGs both on feeding and calving grounds. These "mating groups" aren't limited to the calving grounds, but occur in all parts of the North Atlantic right whale's known migratory range (Kraus \& Hatch 2001). Calving in right whales is highly seasonal in both hemispheres and the estimates of gestation range from 10-13 months (Best, 1994). Therefore, mating that leads to pregnancy is expected to be taking place at approximately the same time that whales are present on the calving ground.

Group composition is highly variable throughout the year including all-female and all-male groups. All of these different groups have been lumped together under the same label, "surface active groups" in the North Atlantic (Kraus et al., 2001). This descriptive term is a bit broader that the previously used "courtship groups" (Donnelly, 1967) and does not address the function of these groups. This term, surface active group (SAG), will be used to refer to right whale social groups throughout this thesis.

Southern right whale acoustics were extensively studied in Patagonia by Christopher W. Clark in the 1970's (Clark, 1982; Clark, 1984; Clark, 1983; Clark \& Clark, 1980). His work, coupled with some preliminary recording work done by the New England Aquarium of the North Atlantic right whale, provided a solid basis for using the acoustic behavior of right whales to unravel the mystery of the surface active group. Only the humpback whale had been the subject of more acoustic research, and it seemed likely 
that studying acoustic communication in the North Atlantic right whale could address several important issues relating to reproduction and social behavior in the right whale. The goal of this thesis is to describe the acoustic behavior of North Atlantic right whales in surface active groups on their feeding grounds in the Bay of Fundy, Canada. The research questions focus on the composition and occurrence of these groups, the sounds produced by whales in the groups and the response and hearing capabilities of right whales related to acoustic communication.

\subsection{LITERATURE CITED}

Acharya, L. \& McNeil, J. N. 1998. Predation risk and mating behavior: The responses of moths to bat-like ultrasound. Behavioral Ecology, 9, 552-558.

Aguilar, A. 1986. A review of old Basque whaling and its effect on the right whale (Eubalaena glacialis) of the North Atlantic. In: Right Whales: Past and Present status, Special Issue No. 10, (Ed. by Jr., R. L. B., Best, P. B. \& Prescott, J. H.), pp. 191-200. Cambridge, England: International Whaling Commission.

Andersson, M. 1982. Female choice selects for extreme tail length in a widowbird. Nature, 299, 818-820.

Appleby, M. C. 1982. The consequences and causes of high social rank in red deer Cervus elaphus stags. Behaviour, 80, 259-273.

Baker, C. S. 1985. The population structure and social organization of humpback whales (Megaptera novaeangliae) in the central and eastern North Pacific. Ph.D. Thesis. University of Hawaii, Honolulu.

Baker, C. S. \& Herman, L. 1985. Whales that go to extremes. Natural History, 10:, 5260.

Baker, C. S. \& Herman, L. M. 1984. Aggressive behavior between humpback whales, Megaptera novaeangliae, wintering in Hawaiian waters. Canadian Journal of Zoology, 62, 1922-1937.

Baker, C. S. \& Herman., L. 1984. Seasonal contrasts in the social behavior of the humpback whale. Cetus, Vol. 5., 14-16.

Balcomb, K. C., Boran, J. R. \& Heimlich, S. L. 1982. Killer whales in greater Puget Sound. Report of the International Whaling Commission, 32, 681-685. 
Berta, A. \& Sumich, J. L. 1999. Marine mammals: Evolutionary biology. San Diego: Academic Press.

Best, P. B. 1990. Trends in inshore right whale populations off South Africa. 1969-1987. Marine Mammal Science, 6, 93-108.

Best, P. B. 1994. Seasonality of reproduction and the length of gestation in southern right whales Eubalaena australis. Journal of Zoology, London, 232, 175-189.

Best, P. B., Brandao, A. \& Butterworth, D. S. 2001. Demographic parameters of southern right whales off South Africa. Journal of Cetacean Research Management (Special Issue 2), 161-169.

Bigg, M. A., Olesiuk, P. F., Ellis, G. M., Ford, J. K. B. \& Balcomb, K. C. 1990. Social organization and genealogy of resident killer whales (Orcinus orca) in the coastal waters of British Columbia and Washington State. In: Reports of the International Whaling Commission, special issue 12 (Ed. by Hammond, P. S., Mizroch, S. A. \& Donovan, G. P.), pp. 383-405. Cambridge: International Whalin Commission.

Bradbury, J. W. \& Vehrencamp, S. L. 1998. Principles of animal communication. Sunderland, MA: Sinauer Associates, Inc.

Brownell Jr., R. L., Clapham, P. J., Miyashita, T. \& Kasuya, T. 2001. Conservation status of North Pacific right whales. J. Cetacean Res. Manage. (Special Issue 2), 269-286.

Brownell, R. L. J. \& Ralls, K. 1986. Potential for sperm competition in baleen whales. Rep. Int. Whal. Commn. (Special Issue 8), 97-112.

Caswell, H., Fujiwara, M. \& Brault, S. 1999. Declining survival probability threatens North Atlantic right whale. Proceedings of the National Academy of Science, 96, 3308-3313.

Catchpole, C. K. 1980. Sexual selection and the evolution of complex songs among European warblers of the genus Acrocephalus. Behaviour, 74, 149-166.

Catchpole, C. K. \& Slater, P. J. B. 1995. Bird song: Biological themes and variations. Cambridge, England: Cambridge University Press.

Cato, D. H. 1991. Songs of humpback whales: the australian perspective. Memoirs of the Queensland Museum, 30, 277-290.

Cerchio, S. \& Dahlheim, M. 2001. Variation in feeding vocalizations of humpback whales (Megaptera novaeangliae) from southeast Alaska. Bioacoustics, 11, 277 295. 
Clapham, P. J. 1996. The social and reproductive biology of humpback whales: An ecological perspective. Mammal Review, 26, 27-49.

Clapham, P. J., Palsbøll, P. J., Mattila, D. K. \& Vasquez, O. 1992. Composition and dynamics of humpback whale competitive groups in the West Indies. Behaviour, 122, $182-194$.

Clark, A. G., Begun, D. J. \& Prout, T. 1999. Female x male interactions in Drosophila sperm competition. Science, 283, 217-220.

Clark, C. W. 1982. The acoustic repertoire of the southern right whale, a quantitative analysis. Animal Behavior, 30, 1060-1071.

Clark, C. W. 1983. Acoustic communication and behavior of the southern right whale. In: Behavior and communication in whales. American Association for the Advancement of Science Selected Symposium 76 (Ed. by Payne, R. S.), pp. 163198. Boulder, CO.: Westview Press.

Clark, C. W. 1984. Acoustic communication and behavior of southern right whales, Eubalaena australis. National Geographic Society Research Reports, 17, 897 907.

Clark, C. W. \& Clark, J. M. 1980. Sound playback experiments with southern right whales (Eubalaena australis). Science, 207, 663-665.

Connor, R. C. 2000. Group living in whales and dolphins. In: Cetacean Societies: Field studies of dolphins and whales (Ed. by Mann, J., Connor, R. C., Tyack, P. L. \& Whitehead, H.), pp. 199-218. Chicago: The University of Chicago Press.

Connor, R. C., Read, A. J. \& Wrangham, R. 2000. Male reproductive strategies and social bonds. In: Cetacean societies: Field studies of dolphins and whales (Ed. by Mann, J., Connor, R. C., Tyack, P. L. \& Whitehead, H.), pp. 247-269. Chicago: The University of Chicago Press.

Connor, R. C. \& Smolker, R. A. 1985. Habituated dolphins (Tursiops sp.) in Western Australia. Journal of Mammalogy, 66.

Cox, C. R. \& LeBoeuf, B. J. 1977. Female incitation of male competition: A mechanism in sexual selection. The American Naturalist, 111, 317-335.

Croll, D. A., Clark, C. W., Acevedo, A., Tershy, B., Flores, S., Gedamke, J. \& Urban, J. 2002. Only male fin whales sing loud songs. Nature, 417, 809.

Darwin, C. 1871. The descent of man, and selection in relation to sex. Appleton, N.Y. 
Donnelly, B. G. 1967. Observations on the mating behaviour of the southern right whale Eubalaena australis. South African Journal of Science, 63, 176-181.

Doty, G. V. \& Welch, A. 2001. Advertisement call duration indicates good genes for offspring feeding rate in gray tree frogs (Hyla versicolor). Behavioral Ecology and Sociobiology, 49, 150-156.

D'Vincent, C. G., Nilson, R. M. \& Hanna, R. E. 1985. Vocalization and coordinated feeding behavior of the humpback whale in southeastern Alaska. Scientific Report of the Whales Research Institute, Tokyo, 36.

Eberhard, W. G. 1998. Female roles in sperm competition. In: Sperm competition and sexual selection (Ed. by Birkhead, T. R. \& Møller, A. P.), pp. 91-116. New York: Academic Press.

Emlen, S. T. \& Oring, W. L. 1977. Ecology, sexual selection, and the evolution of mating systems. Science, 197, 215-223.

Evans, P. G. H. \& Stirling, I. 2001. Life history strategies of marine mammals. In: Marine Mammals: Biology and Conservation (Ed. by Evans, P. G. H. \& Raga, J. A.), pp. 7-62. New York: Plenum Publishers.

Fisher, R. A. 1930. The genetical theory of natural selection. Oxford: Clarendon Press.

Ford, J. K. B., Ellis, G. M. \& Balcomb, K. C. 2000. Killer whales: The natural history and geneology of Orcinus orca in British Columbia and Washington State, Updated edition. Seattle: The University of Washington Press.

Frankel, A. S., Joseph R. Mobley, Jr. , Louis M. Herman. 1995. Estimation of auditory response thresholds in humpback whales using biologically meaningful sounds. In: Sensory Systems of Aquatic Mammals (Ed. by R.A. Kastelein, J. A. T. a. P. E. M.), pp. 55-70. Woerden, The Netherlands: De Spil Publishers.

Frazer, L. N. \& Mercado III, E. 2000. A sonar model for humpback whale song. IEEE Journal of Oceanic Engineering, 25, 160-182.

Frost, K. J., Russell, R. B. \& Lowry, L. F. 1992. Killer whales, Orcinus orca, in the southeastern Bering Sea: Recent sightings and predation on other marine mammals. Marine Mammal Science, 8, 110-119.

Geist, V. 1971. Mountain Sheep: A Study in behavior and evolution. Chicago: University of Chicago Press. 
George, J. C., Bada, J., Zeh, J., Scott, L., Brown, S. E., O'Hara, T. \& Robert, S. 1999. Age and growth estimates of bowhead whales (Balaena mysticetus) via aspartic acid racemization. Canadian Journal of Zoology, 77, 571-580.

Gerhardt, H. C., Dyson, M. L. \& Tanner, S. D. 1995. Dynamic properties of the advertisement calls of gray tree frogs: patterns of variability and female choice. Behavioral Ecology, 7, 7-18.

Gerhardt, H. C. \& Watson, G. F. 1995. Within-male variability in call properties and female preference in the grey treefrog. Animal Behaviour, 50, 1187-1191.

Gomendio, M., Harcourt, A. H. \& Roldán, E. R. S. 1998. Sperm competition in mammals. In: Sperm competition and sexual selection (Ed. by Birkhead, T. R. \& Møller, A. P.). New York: Academic Press.

Guinee, L. N., Chu, K. \& Dorsey, E. M. 1983. Changes over time in the songs of known individual humpback whales (Megaptera novaeangliae). In: Communication and Behavior of Whales (Ed. by Payne, R. S.), pp. 59-80.

Hafner, G. W., Hamilton, C. L., Steiner, W. W., Thompson, T. J. \& Winn, H. E. 1979. Signature information in the song of the humpback whale. J. Acoust. Soc. Am., 66, 1-6.

Haldiman, J. T. \& Tarpley, R. J. 1993. Anatomy and physiology. In: The bowhead whale (Ed. by Burns, J. J., Montague, J. J. \& Cowles, C. J.), pp. 71-156. Lawrence, KS: Society for Marine Mammalogy, Allen Press, Inc.

Hamilton, P. K. 2002. Associations among North Atlantic right whales. M.S. Thesis. University of Massachusetts Boston, Boston.

Hamilton, P. K. \& Martin, S. M. 1999. A catalog of identified right whales from the Western North Atlantic: 1935 to 1997. pp. 27 pp +382 plates. Boston: New England Aquarium.

Hamilton, W. D. \& Zuk, M. 1982. Heritable true fitness and bright birds: A role for parasites? Science, 218, 384-387.

Harcourt, A. H., Harvey, P. H., Larson, S. G. \& Short, R. V. 1981. Testis weight, body weight and breeding systems in primates. Nature, 293, 55-57.

Helweg, D. A., Frankel, A. S., Jr., J. R. M. \& Herman, L. M. 1992. Humpback whale song: our current understanding. In: Marine Mammal Sensory Systems (Ed. by Thomas, J. A., Kastelein, R. A. \& Supin, A. Y.). New York: Plenum Press. 
Herzing, D. L. 1996. Vocalizations and associated underwater behavior of free-ranging Atlantic spotted dolphins, Stenella frontalis and bottlenose dolphins, Trusiops truncatus. Aquatic Mammals, 22, 61-79.

Hoelzel, R. A., Le Boeuf, B. J., Reiter, J. \& Campagna, C. 1999. Alpha-male paternity in elephant seals. Behavioral Ecology and Sociobiology, 46, 298-306.

Hoogland, J. L. 1998. Why do female Gunnison's prairie dogs copulate with more than one male? Animal Behaviour, 55, 351-359.

IWC. 2001. Report of the workshop on status and trends of western North Atlantic right whales. The Journal of Cetacean Research and Management (Special issue) 2, 61-87.

Janetos, A. C. 1980. Strategies of female mate choice: a theoretical analysis. Behav. Ecol. Sociobiol., 7, 107-112.

Jenni, D. A. \& Collier, G. 1972. Polyandry in the American jacana (Jacana spinosa). Auk, 89, 743-765.

Jennions, M. D. \& Petrie, M. 2000. Why do females mate multiply? A review of genetic benefits. Biological Review, 75, 21-64.

Jurasz, C. M. \& Jurasz, V. P. 1979. Feeding modes of the humpback whale, Megaptera novaeangliae, in southeast Alaska. Scientific Report of the Whales Research Institute Tokyo, 31, 69-83.

Kenagy, G. J. \& Trombulak, S. C. 1986. Size and function of mammalian testes in relation to body size. Journal of Mammalogy, 67, 1-22.

Kevles, B. 1986. Females of the species: Sex and survival in the animal kingdom. Cambridge, MA: Harvard University Press.

Knowlton, A. R. \& Kraus, S. D. 2001. Mortality and serious injury of northern right whales (Eubalaena glacialis) in the western North Atlantic. Journal of Cetacean Research Management (Special Issue) 2, 193-208.

Knowlton, A. R., Kraus, S. D. \& Kenny, R. D. 1994. Reproduction in North Atlantic right whales (Eubalaena glacialis). Canadian Journal of Zoology, 72, 297-305.

Knowlton, N. \& Greenwell, S. R. 1984. The influence of female interests. In: Sperm competition and the evolution of animal mating systems (Ed. by Smith, R. L.). New York: Academic Press.

Koski, W. R., Davis, R. A., Miller, G. W. \& Withrow, D. E. 1993. Reproduction. In: The bowhead whale. Special Publication Number 2. The Society for Marine 
Mammalogy (Ed. by Burns, J. J., Montague, J. J. \& Cowles, C. J.), pp. 239-274. Lawrence, KS: Allen Press, Inc.

Kraus, S. D. 1991. Mating strategies in the North Atlantic right whale (Eubalaena glacialis). University of Massachusetts, Boston, MA.

Kraus, S. D., Hamilton, P. K., Kenney, R. D., Knowlton, A. R. \& Slay, C. K. 2001. Reproduction parameters of the North Atlantic right whale. The Journal of Cetacean Research and Management. (Special issue) 2, 231-236.

Kraus, S. D. \& Hatch, J. J. 2001. Mating strategies in the North Atlantic right whale (Eubalaena glacialis). The Journal of Cetacean Research and Management (Special issue) 2, 237-244.

Kraus, S. D., Moore, K. E., Price, C. A., Crone, M. J., Watkins, W. A., Winn, H. E. \& Prescott, J. H. 1986. The use of photographs to identify individual North Atlantic right whales (Eubalaena glacialis). Report of the International Whaling Commission (Special Issue) 10, 145-151.

Lehmann, G. U. C. \& Heller, K. G. 1998. Bushcricket song structure and predation by the acoustically orienting parasitoid fly Therobia leonidei (Diptera: Tachinidae: Ormiini). Behavioral Ecology and Sociobiology, 43, 239-245.

Linklater, W. L., Cameron, E. Z., Minot, E. O. \& Stafford, K. J. 1999. Stallion harassment and the mating system of horses. Animal Behaviour, 58, 295-306.

Lott, D. F. 1979. Dominance relations and breeding rate in mature male American bison. Zeitschrift Für Tierpsychologie, 49, 418-432.

Malik, S., Brown, M. W., Kraus, S. D. \& White, B. N. 2000. Analysis of mitochondrial DNA diversity within and between North and South Atlantic right whales. Marine Mammal Science, 16, 545-558.

Mattila, D. K., Guinee, L. N. \& Mayo, C. A. 1987. Humpback whale song on a North Atlantic feeding ground. J. Mamm., 68, 880-883.

Møller, A. P. 1989. Ejaculate quality, testes size and sperm production in mammals. Functional Ecology, 3, 91-96.

Møller, A. P. \& Ninni, P. 1998. Sperm competition and sexual selection: a meta-analysis of paternity studies in birds. Behavioral Ecology and Sociobiology, 43, 345-358.

Nerini, M. K., Braham, H. W., Marquette, W. M. \& Rugh, D. J. 1984. Life history of the bowhead whale, Balaena mysticetus (Mammalia: Cetacea). Journal of Zoology, London, 204, 443-468. 
Noad, M. J. 2002. The use of song by humpback whales (Megaptera novaeangliae) during migration off the east coast of Australia. Ph.D. Thesis. University of Sydney, Sydney.

Norris, K. J. 1990. Female choice and the quality of parental care in the great tit Parus major. Behavioral Ecology \& Sociobiology, 27, 275-282.

Norris, K. S., Villa-Ramirez, B., Nichols, G., Würsig, B. \& Miller, K. 1983. Lagoon entrance and other aggregations of gray whales (Eschrichtius robustus). In: Communication and behavior of whales (Ed. by Payne, R.), pp. 259-293. Boulder, CO: Westview Press.

Olsson, M. \& Madsen, T. 2001. Promiscuity in sand lizards (Lacerta agilis) and adder snakes (Vipera berus): Causes and consequences. The Journal of Heredity, 92, 190-197.

Payne, K., Tyack, P. \& Payne., R. 1983a. Progressive changes in the songs of humpback whales (Megaptera noveangliae): A detailed analysis of two seasons in Hawaii. In: Communication and behavior of Whales. (Ed. by Payne, R.), pp. 9-57. Boulder, CO: Westview Press.

Payne, R. 1995. Among whales. New York: Scribner.

Payne, R., Brazier, O., Dorsey, E. M., Perkins, J. S., Rowntree, V. J. \& Titus, A. 1983b. External features in southern right whales (Eubalaena australis) and their use in identifying individuals. In: Communication and behavior of whales (Ed. by Payne, R.), pp. 371 - 445. Boulder, CO: Westview Press.

Payne, R. S. \& Dorsey, E. M. 1983. Sexual dimorphism and aggressive use of callosities in right whale (Eubalaena australis). In: Communication and behavior of whales. (Ed. by Payne, R.), pp. 295-329. Boulder, CO: Westview Press.

Payne, R. S. \& McVay, S. 1971. Songs of humpback whales. Science, 173, 585-597.

Payne, R. S. \& Webb, D. 1971. Orientation by means of long range acoustic signalling in baleen whales. Annals of the New York Academy of Science, 188, 110-141.

Rasmussen, L. E. L. \& Schulte, B. A. 1998. Chemical signals in the reproduction of Asian (Elephas maximus) and African (Loxodonta africana) elephants. Animal Reproductive Science, 53, 19-34.

Reeves, R. R. \& Mitchell, E. 1986. The Long Island, New York, right whale fishery: 1650-1924. In: Right Whales: Past and Present Status, Special Issue No. 10. (Ed. by Jr., R. L. B., Best, P. B. \& Prescott, J. H.), pp. 221-254. Cambridge, England: International Whaling Commission. 
Rice, D. W. 1998. Marine mammals of the world: Systematics and distribution.

Lawrence, KS: Society for Marine Mammalogy, Special Publication Number 4.

Richardson, W. J., Finley, K. J., Miller, G. W., Davis, R. A. \& Koski, W. R. 1995. Feeding, social and migration behavior of bowhead whales, Balaena mysticetus, in Baffin Bay vs. the Beufort Sea - regions with different amounts of human activity. Mar. Mam. Sci, 11, 1-45.

Robert, D., Amoroso, J. \& Hoy, R. 1992. The evolutionary convergence of hearing in a parasitoid fly and its cricket host. Science, 258, 1135-1137.

Rosenbaum, H. C., Brownell Jr., R. L., Brown, M. W., Schaeff, C., Portway, V., White, B. N., Malik, S., Pastene, L. A., Patenaude, N. J., Baker, C. S., Goto, M., Best, P. B., Clapham, P. J., Hamilton, P. K., Moore, M., Payne, R., Rowntree, V., Tynan, C. T., Bannister, J. L. \& DeSalle, R. 2000. World-wide genetic differentiation of Eubalaena: Questioning the number of right whale species. Molecular Ecology, 9, 1793-1802.

Sakaluk, S. K. 1986. Sperm competition and the evolution of nuptial feeding behavior in the cricket Gryllodes supplicans. Evolution, 40, 584-593.

Samaras, W. F. 1974. Reproductive behavior of the gray whale (Eschrichtius robustus) in Baja California. Bulletin of the Southern California Academy of Sciences, 73, 5764.

Scott, M. D., Wells, R. S. \& Irvine, A. B. 1990. A long-term study of bottlenose dolphins on the west coast of Florida. In: The Bottlenose Dolphin (Ed. by Leatherwood, S. \& Reeves, R. R.). San Diego: Academic Press, Inc.

Silber, G. K. 1986. The relationship of social vocalizations to surface behavior and aggression in the Hawaiian humpback whale (Megaptera novaeangliae). Canadian Journal of Zoology, 64, 2075-2080.

Silber, G. K., Newcomer, M. W. \& Perez-Cortes, H. M. 1990. Killer whales (Orcinus orca) attack and kill a Bryde's whale (Balaenoptera edeni). Canadian Journal of Zoology, 68.

Steltner, H., Steltner, S. \& Sergeant, D. E. 1984. Killer whales, Orcinus orca, prey on narwhals, Monodon monoceros: An eyewitness account. Canadian Field Naturalist, 98.

Swartz, S. L. 1986. Gray whale migratory, social and breeding behavior. Report of the International Whaling Commission, Special Issue 8, 207-229. 
Tormosov, D. D., Mikhalev, Y. A., Best, P. B., Zemsky, V. A., Sekiguchi, K. \& Brownell Jr., R. L. 1998. Soviet catches of southern right whales, Eubalaena australis, 1951-1971; biological data and conservation implications. Biological Conservation, 86, 185-197.

Tyack, P. 1983. Differential response of humpback whales, Megaptera novaeangliae, to playback of song or social sounds. Behavioral Ecology and Sociobiology, 13, 4955 .

Tyack, P. 1986. Population biology, social behavior and communication in whales and dolphins. TREE, 1, 144-150.

Tyack, P. \& Whitehead, H. 1983. Male competition in large groups of wintering humpback whales. Behaviour, 83, 132-154.

Tyack, P. L. 1982. Humpback whales respond to sounds of their neighbors. Ph.D. The Rockefeller University, New York.

Vincent, A. C. J. \& Sadler, L. M. 1995. Faithful pairbonds in wild seahorses, Hippocampus whitei. Anim. Behav., 50, 1557-1569.

Warner, R. R. \& Dill, L. M. 2000. Courtship displays and coloration as indicators of safety rather than of male quality: the safety assurance hypothesis. Behavioral Ecology, 11, 444-451.

Weinrich, M. T. 1991. Stable social associations among humpback wales (Megaptera novaeangliae) in the southern Gulf of Maine. Canadian Journal of Zoology, 69, 3012-3019.

Wells, K. D. 1977. Territoriality and male mating success in the green frog Rana clamitans. Ecology, 58, 750-762.

Whitehead, H. \& Mann, J. 2000. Female reproductive strategies of cetaceans: Life histories and calf care. In: Cetacean societies: Field studies of dolphins and whales (Ed. by Mann, J., Connor, R. C., Tyack, P. L. \& Whitehead, H.), pp. 219246. Chicago: The University of Chicago Press.

Wilmer, J. W., Allen, P. J., Pomeroy, P. P., Twiss, S. D. \& Amos, W. 1999. Where have all the fathers gone? An extensive microsatellite analysis of paternity in the grey seal (Halichoerus grypus). Molecular Ecology, 8, 1417-1429.

Wilson, N., Tubman, S. C., Eady, P. E. \& Robertson, G. W. 1997. Female genotype affects male success in sperm competition. Proceedings of the Royal Society of London B, 264, 1491-1495. 
Winn, H. E., Price, C. A. \& Sorensen, P. W. 1986. The distributional biology of the right whale (Eubalaena glacialis) in the western North Atlantic. Report of the International Whaling Commission. (Special issue), 10, 129-138.

Winn, H. E. \& Winn, L. K. 1978. The song of the humpback whale Megaptera novaeangliae in the West Indies. Marine Biology, 47, 97-114.

Würsig, B. 1986. Delphinid foraging strategies. In: Dolphin cognition and behavior: a comparative approach (Ed. by Schusterman, R. J., Thomas, J. A. \& Woods, F. G.), pp. 347-359. Hillsdale, NJ: Lawrence Erlbaum Associates, Inc.

Würsig, B. \& Clark, C. W. 1993. Behavior. In: The bowhead whale (Ed. by Burns, J. J., Montague, J. J. \& Cowles, C. J.), pp. 157-199. Lawrence, KS.

Würsig, B. \& Würsig, M. 1980. Behavior and ecology of the dusky dolphin, Lagenorhynchus obscurus in the south Atlantic. Fishery Bulletin, 77, 871-890.

Yamane, A. 1999. Male homosexual mounting in group-living feral cats (Felis catus). Ethology, Ecology \& Evolution, 11, 399-406.

Yasukawa, K. 1981. Male quality and female choice of mate in the red-winged blackbird Agelaius phoeniceus. Ecology, 62, 922-929.

Zahavi, A. 1975. Mate selection - a selection for a handicap. Journal of Theoretical Biology, 53, 205-214.

Zeh, J. A. \& Zeh, D. W. 1997. The evolution of polyandry II: post-copulatory defenses against genetic incompatibility. Proceedings of the Royal Society of London B, 264, 69-75. 


\section{ChAPTER 2. SOCIAL BEHAVIOR OF NORTH ATLANTIC RIGHT WHALES (EUbALAENA GLACIALIS) IN SURFACE ACTIVE GROUPS}

\subsection{INTRODUCTION}

The North Atlantic right whale (Eubalaena glacialis) is a highly endangered species of baleen whale. Hunted to near extinction in the 1600's by Basque whalers (Aguilar, 1986) the right whale population in the Western North Atlantic has shown little appreciable recovery since it received complete protection from whaling in 1935 (Brownell et al., 1986). As a result, this population has come under intense scrutiny from a management perspective. Most current research focuses on the two aspects of right whale biology most crucial to the recovery of this population; reproduction and mortality. Unfortunately, twenty years of research indicate that anthropogenic causes of mortality (including entanglement in fishing gear and collisions with vessels) continue to threaten the population (Knowlton \& Kraus, 2001). The reproductive output remains highly variable year to year but remains low overall (Kraus et al., 2001). The population size was determined to be decreasing in the 1990's (Caswell et al., 1999) though a recent increase in the calving rate provides some hope for the future. With this understandable focus on the basic demographic information and survival of the species, relatively little research has been directed to answer general questions about behavior in this species. Behavioral observations can provide insight into the two primary questions about North Atlantic right whale biology, including possibly the largest puzzle remaining: where and when do right whales mate? Observations of the locations and timing of behaviors related to reproduction may shed light on this subject.

The surface active group (SAG) is one of the most striking aspects of right whale behavior that is thought to be related to reproduction. SAGs in the North Atlantic right whale are defined as two or more whales interacting at the surface with frequent body contact (Kraus \& Hatch, 2001). Many SAGs are described as groups with a single central female, called the focal female, flanked by 1-32 male right whales competing with one 
another to get close enough to mate with the female. Current evidence on sound production and group structure suggests that the formation and maintenance of these groups is controlled by the female (Kraus \& Hatch, 2001). This observation raises several interesting questions about social structure and mating behavior in this species (Kraus \& Hatch, 2001). Does the female control mate choice by calling multiple males in and forcing them to compete for access to mating? More importantly, do these groups have any direct connection to reproduction in this species?

Right whale reproductive anatomy has led several researchers to speculate on mating strategy in this species. Males that compete via sperm competition typically have relatively larger testes and longer penises than males in species where females only copulate with one male (Kenagy \& Trombulak, 1986; Møller, 1989; Harcourt et al., 1981; Møller \& Ninni, 1998). Male right whales have long fibroelastic penises (2-3 $\mathrm{m}$ in length) and large testes (Mean $=843 \mathrm{~kg}, \mathrm{Std}=160 \mathrm{~kg}, \mathrm{~N}=4$ (Omura et al., 1969)). These two traits indicate that sperm competition plays a role in right whale reproduction (Brownell \& Ralls, 1986). These anatomical features allow males to produce large volumes of sperm to flood the female reproductive tract to displace or dilute any sperm from previous copulations. The longer penis allows for sperm to be delivered closer to the egg (Møller, 1998). Behavioral observations of SAGs frequently involve multiple males achieving intromission with a single female. This further supports the theory of sperm competition in right whales.

SAGs have been reported in all known right whale habitats during eight months of the year based upon observations from 1981-1990 (Kraus \& Hatch, 2001). The lack of SAG sightings reported from June, November, December and January might primarily be a result of low numbers of right whale sightings in these months. Sighting records from the past ten years have documented SAGs in all months of the year except November. Sightings of right whales remain very low in the month of November (right whale consortium unpublished data). Even though SAGs are seen throughout the year, calving is highly seasonal, with calves being born in the winter months (Kraus et al., 1986b). Therefore SAGs commonly observed on the summer feeding grounds do not lead directly 
to fertilization unless right whales are capable of delayed implantation. The current estimate of the gestation length for Southern right whales (12-13 months) (Best, 1994) would have to underestimate the gestation in North Atlantic right whales by more than four months. Groups engaged in social behavior, including SAGs, are commonly observed (up to $41 \%$ of sightings (Hamilton, 2002)) and may take up a significant portion of an individual's time on any given day. Reproduction appears to be an obvious function, but the timing of SAG occurrence suggests that fertilization alone is unlikely to be the only functions of these groups. It is possible that SAGs serve multiple reproductive and social functions in this species, including, but not limited to fertilization.

Although many species of mammals only engage in sexual behavior for reproduction, it is not uncommon for sexual activity to take place for other reasons. Sexual behavior not related directly to conception has been described for many mammalian species. Sexual behavior is commonly used for reconciliation or strengthening of social bonds in primate species (Hrdy \& Whitten, 1987; Call et al., 1999). Several cetacean species, for example the bottlenose dolphin (Tursiops truncatus) and the spinner dolphin (Stenella longirostris), show high levels of sexual activity, often directed at members of the same sex (or inanimate objects) (Connor et al., 2000; Nunn et al., 2000). There are typically costs associated with mating ranging from direct injury to transmission of disease (Jennions \& Petrie, 2000; Nunn et al., 2000). Therefore, it is likely that sexual activity unrelated to conception has some benefit to individuals in these species, possibly in terms of social bonding.

It is possible that SAGs in right whales function directly for fertilization or that they represent interactions between individuals that may be important for reproduction or survival at a later date. The general definition of a SAG may encompass both of these interactions under one label. One way to gain a better understanding of which functions are the most likely explanations for SAGs is to first determine when and where they occur, what group compositions can be found in different areas and how individual participation in SAGS may be dependent on relatedness to other whales in the group. This paper will summarize the sightings of SAGs from 1/1/1992-12/31/2001 and 
describe group composition, location and timing of surface activity. These analyses show that 1) group composition varies with location and time of year and that 2) patterns of male and female participation in SAGS are distinctly different.

\subsection{METHODS}

\subsubsection{Sighting Catalog Database}

The population of right whales in the western North Atlantic has been intensively studied for over 20 years. This long term research effort, started by the New England Aquarium, has resulted in a large database of information including details about sex, age and reproductive history for many individuals as well as general demographic information (Hamilton \& Martin, 1999). Individual right whales can be identified by distinct callosity and scarring patterns (Payne \& Dorsey, 1983; Kraus et al., 1986a). A central database maintained by the New England Aquarium for the North Atlantic right whale consortium contains photographic sighting records for 436 individual right whales contributed by numerous organizations and individuals from the last 23 years (Hamilton \& Martin, 1999). This database makes it possible to determine the sex, age, and matrilineal relatedness of individuals non-invasively from photographs of their callosity and scarring patterns. Detailed questions about group composition can be answered through analysis of existing data.

For this chapter, data on the distribution, timing, and individual composition of SAGs were taken from the North Atlantic right whale consortium database. Queries of this database produced absolute numbers and locations of SAGs seen in given years, number

of SAGs per month divided by the number of distinct individuals identified in that month, minimum number of whales in each group photographed over the course of observation and the compositions of many groups in which all animals were photographed.

There are five main regions where sighting data were collected between 1992 and 2001. These five regions include three critical habitat areas located in U.S. waters (Southeastern United States, Massachusetts Bay, and the Great South Channel) and two 
conservation areas located in Canadian waters (Bay of Fundy and Roseway Basin) (Hamilton, 2002). Sighting effort in these five regions varied year to year between 1992 and 2001, with the Southeastern United States and the Bay of Fundy regions receiving the most consistent coverage. A description of survey efforts over the past ten years can be found in Hamilton (2002).

\subsubsection{Corrections for right whale occurrence}

Typically, when data from surveys are analyzed, corrections are made for sightings per unit effort (SPUE) (e.g. (Kishino \& Kasamatsu, 1987). However, the number of individuals present in a given location at a given time should have more influence on the occurrence of social behavior than observer effort. For example, if there were 20 right whale sightings in a region during six survey days in a given month, there would be very different behavioral implications for 20 replicate sightings of 4 individuals as opposed to 20 sightings of 19 or 20 different individuals over the month, even if the sightings per unit effort were the same. Therefore the counts of SAG sightings were corrected for the total number of different individuals seen in a given month in a given region, rather than correcting for survey effort, or miles of track-line flown. The number of SAGs sighted in a given month and region $\left(N_{S A G}^{D R}\right)$ and the number of individuals seen in the same month and region $\left(N_{I D}^{D R}\right)$ were used to calculate a correction factor $\left(N_{S A G}^{C o r r}=N_{S A G}^{D R} / N_{I D}^{D R}\right)$. This correction also controls for differences in survey effort between years due to weather or funding. An additional correction was done based on the total number of sightings in a given month $\left(N_{\text {sightings }}^{\text {Total }}\right)$ and the total number of sightings of whales in SAGs $\left(N_{\text {Sightings }}^{\text {SAG }}\right)$. These two values were used to calculate the proportion of sightings in a given month that were of whales in SAGs $\left(N_{\text {sightings }}^{\text {Corr }}=N_{\text {Sightings }}^{\text {SAG }} / N_{\text {Sightings }}^{\text {Total }}\right)$. 


\subsubsection{Analyses of catalog data}

Analyses of the SAG sighting database included the location, date, group size and composition of each SAG sighting. Each SAG for which all individuals were identified was grouped into one of seven categories of group type (Table 2.1) or labeled as an eighth unknown group type. The occurrence of the seven group compositions was considered annually and by month. Individual involvement in SAGs was considered in terms of total numbers of sightings, proportion of total sightings, and the age of first participation in SAGs.

Table 2.1. Description of the SAG categories based on group composition. $\mathrm{R}=$ Potentially reproductive, $\mathrm{NR}=$ Non-reproductive. Note that the designation of group type for the calf group and the pregnant female group takes precedence over other group types. This may result in groups being omitted from other appropriate categories (i.e. a group with a calf and three females could also be an all female group or a group with multiple females with males, but to investigate the social role of calves in SAGs, these groups were kept separate from the other categories.)

*-A pregnant female is defined as a female sighted in a SAG less than 12 months before she was known to have given birth to a calf.

\begin{tabular}{|c|c|c|}
\hline Group Type & Description & Function \\
\hline Male-Female Pair & Pairs of animals where one animal of each sex was present & $\mathrm{R}$ \\
\hline $\begin{array}{l}\text { One Female with } \\
\text { Multiple Males }\end{array}$ & $\begin{array}{l}\text { One individual in the group was female while all other sighted } \\
\text { animals were male }\end{array}$ & $\mathrm{R}$ \\
\hline $\begin{array}{l}\text { Multiple Females } \\
\text { with Males }\end{array}$ & $\begin{array}{l}\text { More than one individual in the group was female and at least one } \\
\text { male was also present }\end{array}$ & $\mathrm{R}$ \\
\hline All Female & All individuals sighted in the group were female & NR \\
\hline All Male & All individuals sighted in the group were male & NR \\
\hline Calf & $\begin{array}{l}\text { Any SAG with a calf recorded in the group regardless of the age } \\
\text { or sex of any other animals in the group }\end{array}$ & $\mathrm{NR} / \mathrm{R}$ \\
\hline Pregnant female & $\begin{array}{l}\text { Any group with a known pregnant* female regardless of the age } \\
\text { and sex of any other animals in the group }\end{array}$ & NR \\
\hline Unknown & At least one animal in the group is of unknown sex & Unknown \\
\hline
\end{tabular}




\subsubsection{Occurrence of $S A G S$}

All sighting records labeled SAG in the database, based on the definition of two or more individuals interacting at the surface with frequent physical contact, were considered to be part of a SAG. Groups were distinguished by the date, time, latitude and longitude indicated for each sighting record. Each group was given a number by year and the total number of groups was calculated.

Counts of group size were made where group size was defined as the minimum number of whales identified in each SAG. SAGs in which all whales were identified were assigned into a particular group type, depending on the age and sex of the individuals in the group (Table 2.1). SAGs containing unidentified individuals or individuals whose gender was unknown were assigned to an "unknown" category. After the assignment of group size and type was made, the distribution of these different group types was determined by month and region. A summary of the data collected for each SAG is presented in Table 2.2 .

Table 2.2. A list of data collected for each SAG sighting record.

Data collected for each SAG

\section{Month}

Year

Region

Group Size

Individuals in group

Group type

Month of the sighting.
Year of the sighting.
The survey region that the SAG was observed.
Minimum number of individuals documented in the SAG
sighting record.
Individual ID numbers from the right whale consortium
database catalog (RWCDC).
Type of SAG based on individuals seen in the group

Year of the sighting.

The survey region that the $S A G$ was observed.

Minimum number of individuals documented in the SAG sighting record. database catalog (RWCDC).

Type of SAG based on individuals seen in the group

The sighting histories of individual whales in SAGs were analyzed to determine total number of SAG sightings per individual, proportion of total sightings in SAGs by individual, total and proportion of SAG sightings by sex for animals of known sex, and age at first sighting for all whales of both known age and known sex. Additionally, detailed sighting histories for four whales all born in the same year are described in Appendix 1 to emphasize the large variation in observed behavior between individuals given the same annual environmental conditions. All data on individual sighting histories 
rely on the entire historical sighting database and include all recorded sightings for all individuals in the North Atlantic right whale catalog (Hamilton \& Martin 1999) and all individuals added to the catalog since its publication through 2001. A summary of the data used for individual whales is summarized in Table 2.3.

Table 2.3. A list of data collected for each individual related to SAG participation. Not all information was available for all whales. Age and sex are unknown for several individuals.

Data collected for each individual

\begin{tabular}{ll}
\hline Age & Age in years. \\
Sex & $\begin{array}{l}\text { Gender of the whale if known. } \\
\text { Total number of sightings recorded in the RWCDC for the } \\
\text { individual for all years. }\end{array}$ \\
Number of sightings in SAGs & $\begin{array}{l}\text { Total number of sightings in the RWCDC of the individual in a } \\
\text { SAG. }\end{array}$ \\
Proportion of sightings in SAGs & $\begin{array}{l}\text { Number of sightings in SAGs divided by the total number of } \\
\text { sightings. } \\
\text { Age in years at the first documented sighting in a SAG if } \\
\text { known. }\end{array}$ \\
\hline
\end{tabular}

\subsubsection{Annual number of $S A G s$ related to calf output}

Simple linear correlations were calculated to test for any relationship between the number of SAGs seen during different times of the year and calf production. Calves that are born from December $1-$ February $28^{\text {th }}$ are considered to be in the same cohort. For example, the 2001 calves $(\mathrm{N}=30)$ were born between December 1, 2000 and February 28, 2001. It is expected that conception should occur 12-13 months before calving. Therefore, SAG activity leading up to and resulting in conception and implantation of an embryo should be observed 12-14 months before calving (i.e. SAGs from November 1, 1999- January 31, 2000 should result in the calves born in the 2001 season). Correlations between the number of calves born in a cohort and the number of SAGs observed 1) 1214 months before their birth and 2) 0-11 months before their birth were considered. The correlation between the number of each type of SAG (either R or NR from Table 2.1) and calf production was also calculated. 


\subsection{RESULTS}

\subsubsection{Occurrence of SAGs}

A total of 19,133 sightings of individually photo-identified right whales from 1/1/1992$12 / 31 / 2001$ were used in this analysis. Of those sightings, the total number of sightings of whales in SAGs was 3393, or approximately $18 \%$ of the sightings. Sightings from a SAG were recorded with the same date, time and position. Each group was assigned a different number. The total number of SAGs was 918 (Table 2.4), lower than the number of sightings because multiple sightings of individual whales made up each group. Survey data were included from all months. The total number of sightings for November was quite low with a total of 7 sightings of the 19,133 from November.

The total number of SAGs sighted per year varied between a low of 29 and a high of 149 (Mean 91.8, Std 44.4) (Figure 2.1). The average number of SAG sightings per month varied. The highest numbers of SAGs were sighted in August and September, with very few SAGs sighted in May, June and November (Figure 2.2). The observed peaks in August and September for the non-standardized data may not accurately reflect a total increase in SAG activity as these were the months with the greatest sighting effort and highest total number of right whale sightings. The monthly averages with the total number of SAGS sighted in a given month divided by the total number of individual whales sighted in that month are shown in Figure 2.3. A correction of SAG observations as a percentage of total sightings to compare to the whale/month standardization also eliminates the single high peak of surface activity in August and September (Figure 2.4). 
Chapter 2: Social behavior in right whales

Table 2.4. The total number of SAGS sighted by month in each of the ten years.

\begin{tabular}{c|ccccccccccccc}
\hline Year & Jan & Feb & Mar & Apr & May & Jun & Jul & Aug & Sep & Oct & Nov & Dec $\begin{array}{c}\text { Total } \\
\text { by }\end{array}$ \\
\hline 1992 & 4 & 2 & 3 & 5 & 0 & 0 & 0 & 14 & 17 & 7 & 0 & 0 & 52 \\
1993 & 11 & 8 & 8 & 0 & 0 & 0 & 0 & 20 & 6 & 0 & 0 & 9 & 62 \\
1994 & 5 & 0 & 0 & 0 & 0 & 0 & 1 & 57 & 27 & 0 & 0 & 0 & 90 \\
1995 & 1 & 0 & 16 & 1 & 0 & 0 & 5 & 67 & 43 & 10 & 0 & 6 & 149 \\
1996 & 35 & 10 & 20 & 15 & 0 & 1 & 6 & 19 & 15 & 0 & 0 & 1 & 122 \\
1997 & 4 & 6 & 3 & 0 & 0 & 0 & 2 & 45 & 9 & 11 & 0 & 2 & 82 \\
1998 & 11 & 13 & 13 & 3 & 1 & 0 & 0 & 5 & 2 & 0 & 0 & 0 & 48 \\
1999 & 2 & 0 & 2 & 0 & 3 & 0 & 1 & 13 & 7 & 0 & 0 & 1 & 29 \\
2000 & 3 & 14 & 16 & 3 & 0 & 2 & 21 & 56 & 16 & 1 & 0 & 3 & 135 \\
2001 & 10 & 20 & 9 & 2 & 2 & 1 & 21 & 50 & 34 & 0 & 0 & 0 & 149 \\
\hline Total by & 86 & 73 & 90 & 29 & 6 & 4 & 57 & 346 & 176 & 29 & 0 & 22 & 918 \\
month & & & & & & & & & & & & & \\
Average \# & 8.6 & 7.3 & 9 & 2.9 & 0.6 & 0.4 & 5.7 & 34.6 & 17.6 & 2.9 & 0 & 2.2 & 91.8 \\
SAGs/year & & & & & & & & & & & & &
\end{tabular}

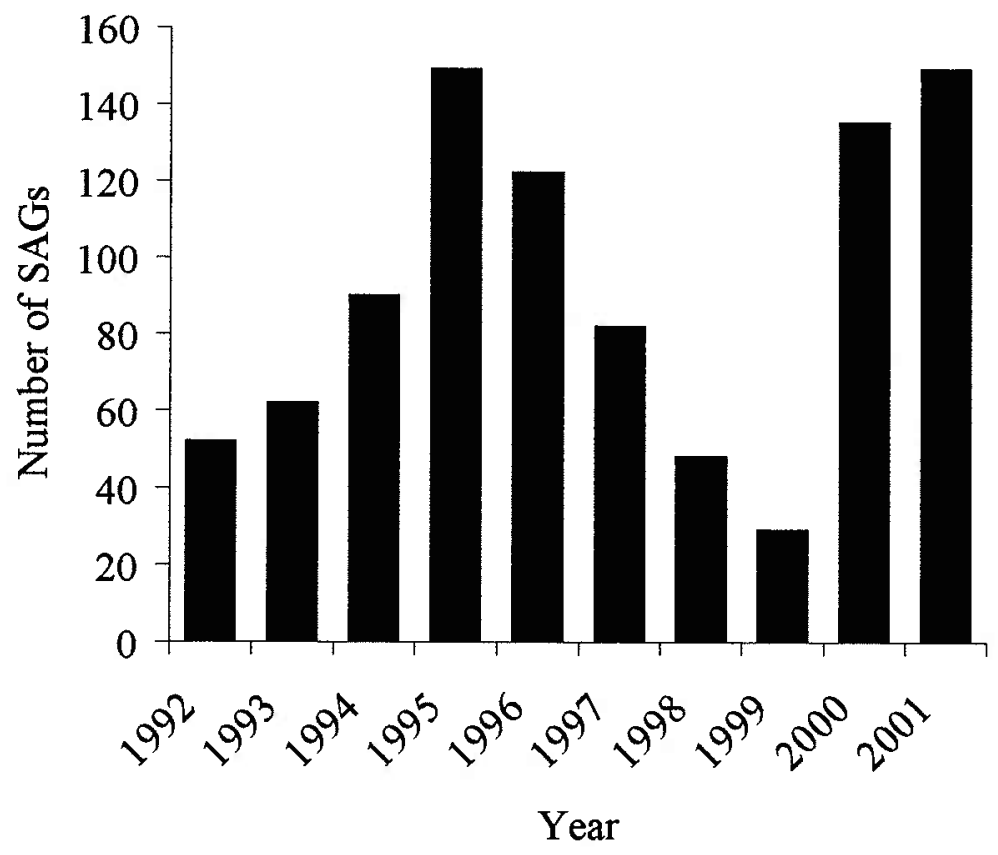

Figure 2.1. Number of SAGS sighted per year from 1992-2001. 


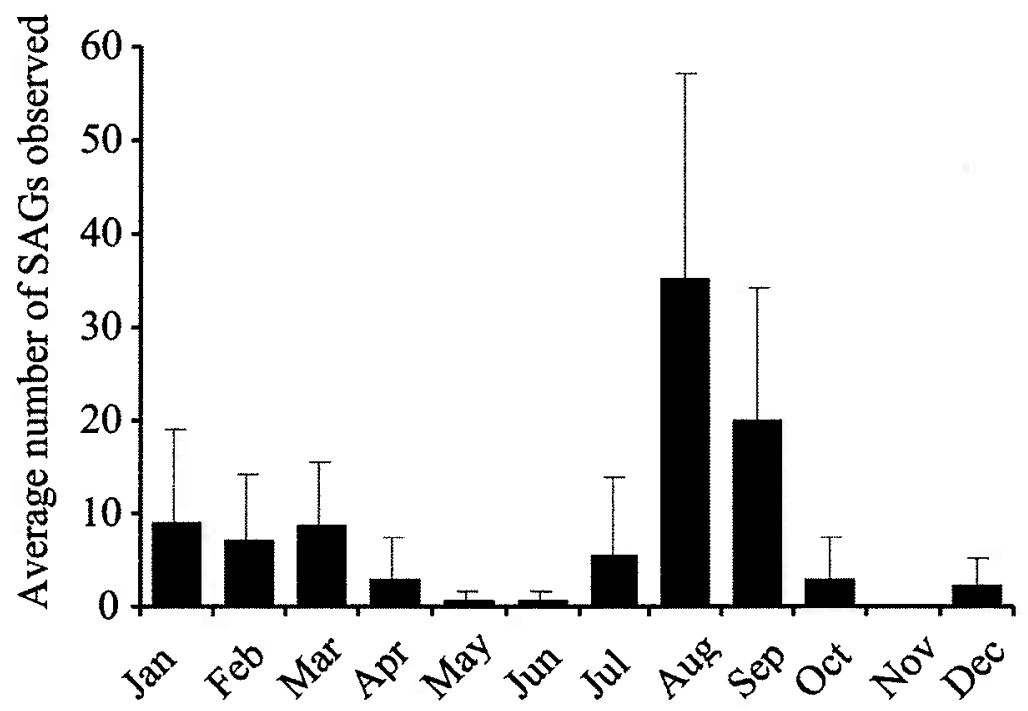

Figure 2.2. Average number of SAGs sighted per month with error bars showing 1 Std between 1/1/1992 and 12/31/2001.

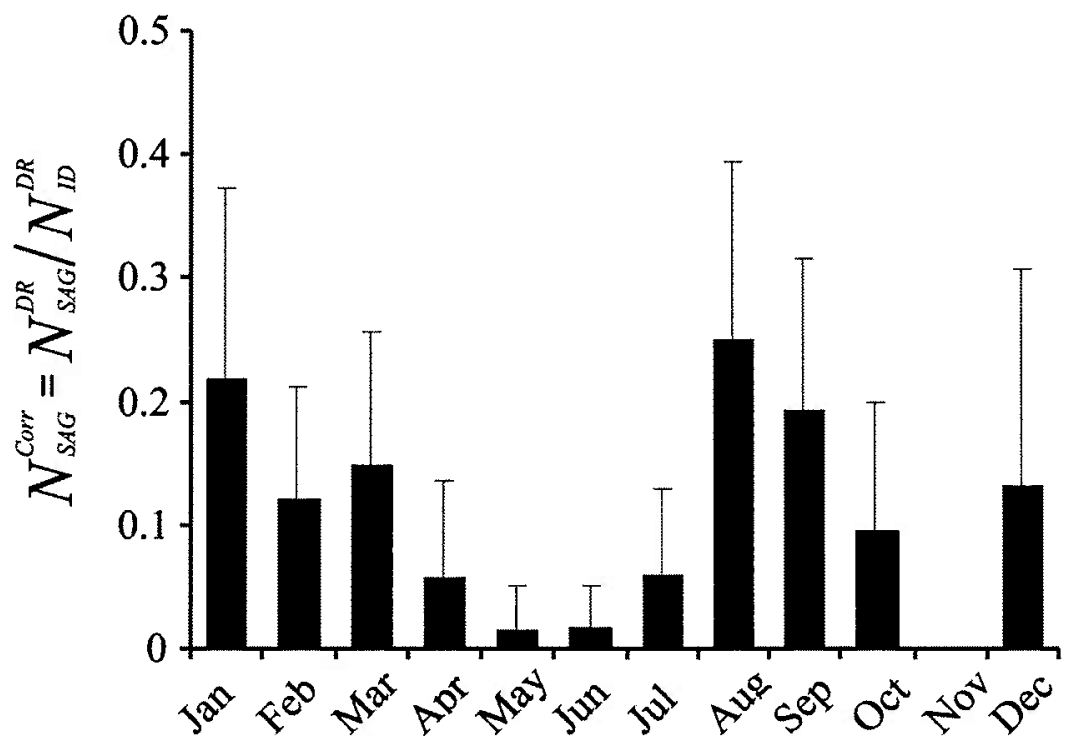

Figure 2.3. Average of number of SAGs seen each month $\left(N_{S A G}^{D R}\right)$ divided by the total number of individual whales sighted in that month $\left(N_{I D}^{D R}\right)$ with error bars showing 1 Std between $1 / 1 / 1992$ and $12 / 31 / 2001$. 


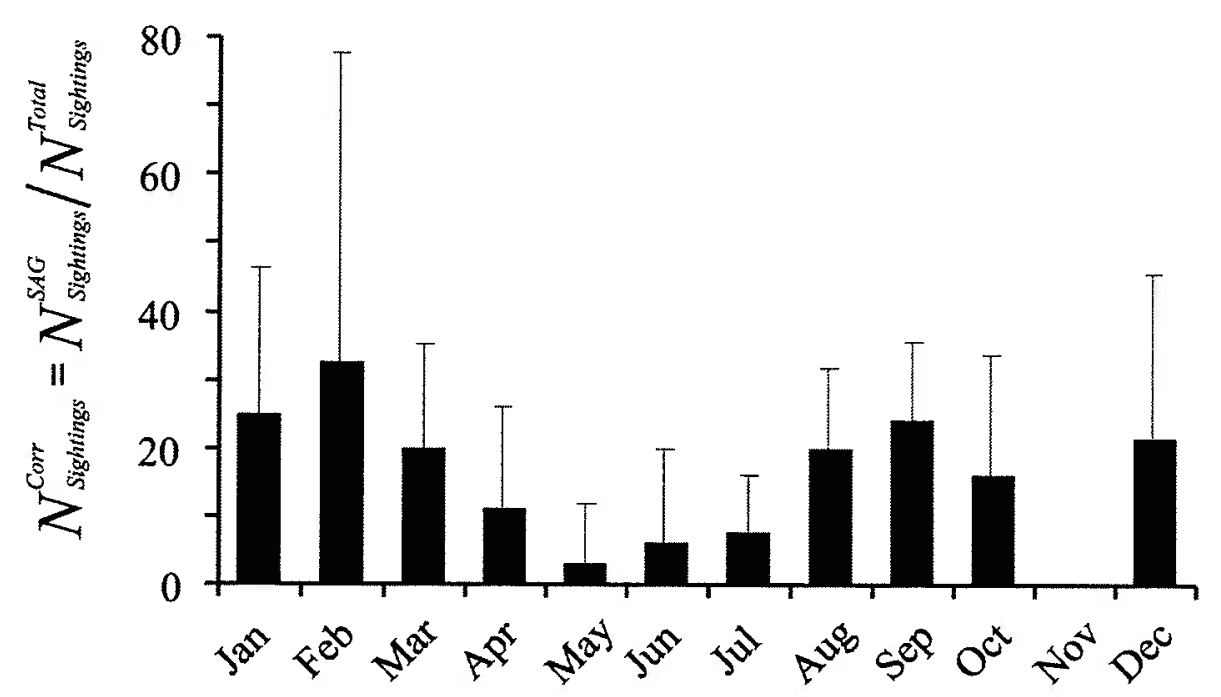

Figure 2.4. Percentage of SAG sightings ( $N_{\text {Sightings }}^{S A G}$ ) from the total number of right whale sightings made each month $\left(N_{\text {Sightings }}^{\text {Total }}\right)$ averaged from 1/1/1992 to 12/31/2001.

\subsubsection{Group Size and Composition}

The number of individuals sighted in each SAG is assumed to be a minimum count of animals present in the group, due to the inherent difficulties of identifying all individuals in SAGS where some animals may remain submerged for long periods of time. In larger groups the group composition is fluid, with individuals leaving and joining the groups as time progresses. This may prevent photographers from photographing all individuals adequately to make a confident assignment of $\mathrm{ID}$ in larger groups. The range of group size was from 2 to 40 individuals (Mean 3.7, $\operatorname{Std} 2.8, \mathrm{~N}=918$ ) for all SAGS. The average group size did not vary significantly by month.

For 677 of these 918 SAGS, the identities and sex of all individuals sighted were recorded and were believed to identify all members present in the group. These 677 groups could be broken down into the seven group types (Table 2.1) based on the group composition. The remaining 241 groups either had sighted individuals that were not identifiable due to poor quality of photographs or the individuals were of unknown sex and these groups were assigned to the unknown group category. 


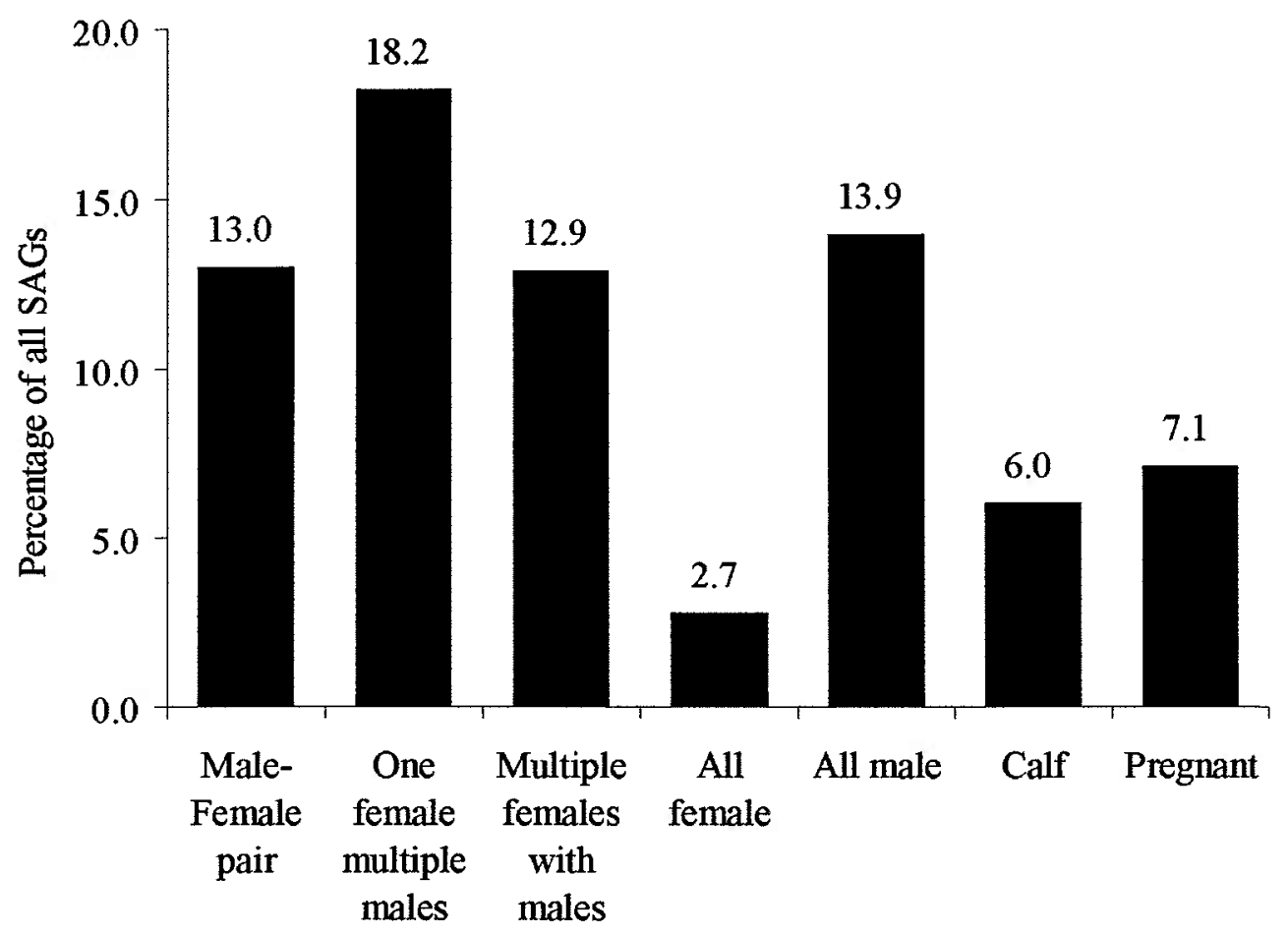

Figure 2.5. The proportion of all 677 identified SAGS that fell into each of the seven categories.

The proportion of SAG sightings of each group type from the 677 SAGs are shown in Figure 2.5. The most common SAG composition described in Kraus \& Hatch (2001) for the Bay of Fundy through 1990, a single female with multiple males, is still more common than any of the other 6 known categories though many of the other categories are well represented when sightings for all regions are combined.

The timing of group type by month (Figure $2.6 \& 2.7$ ) and region (Figure 2.8) also varies, with different group types making up different proportions of the total number of SAGs sighted in different months and regions. There is a notable increase in all-male groups in the late summer and early fall. SAGs with calves are much more common in the summer months than the few months after birth. There is a peak in pregnant female groups in the southeastern U.S. in December, shortly before these females give birth. 


\section{Reproductive Groups}

A) Male-Female Pair

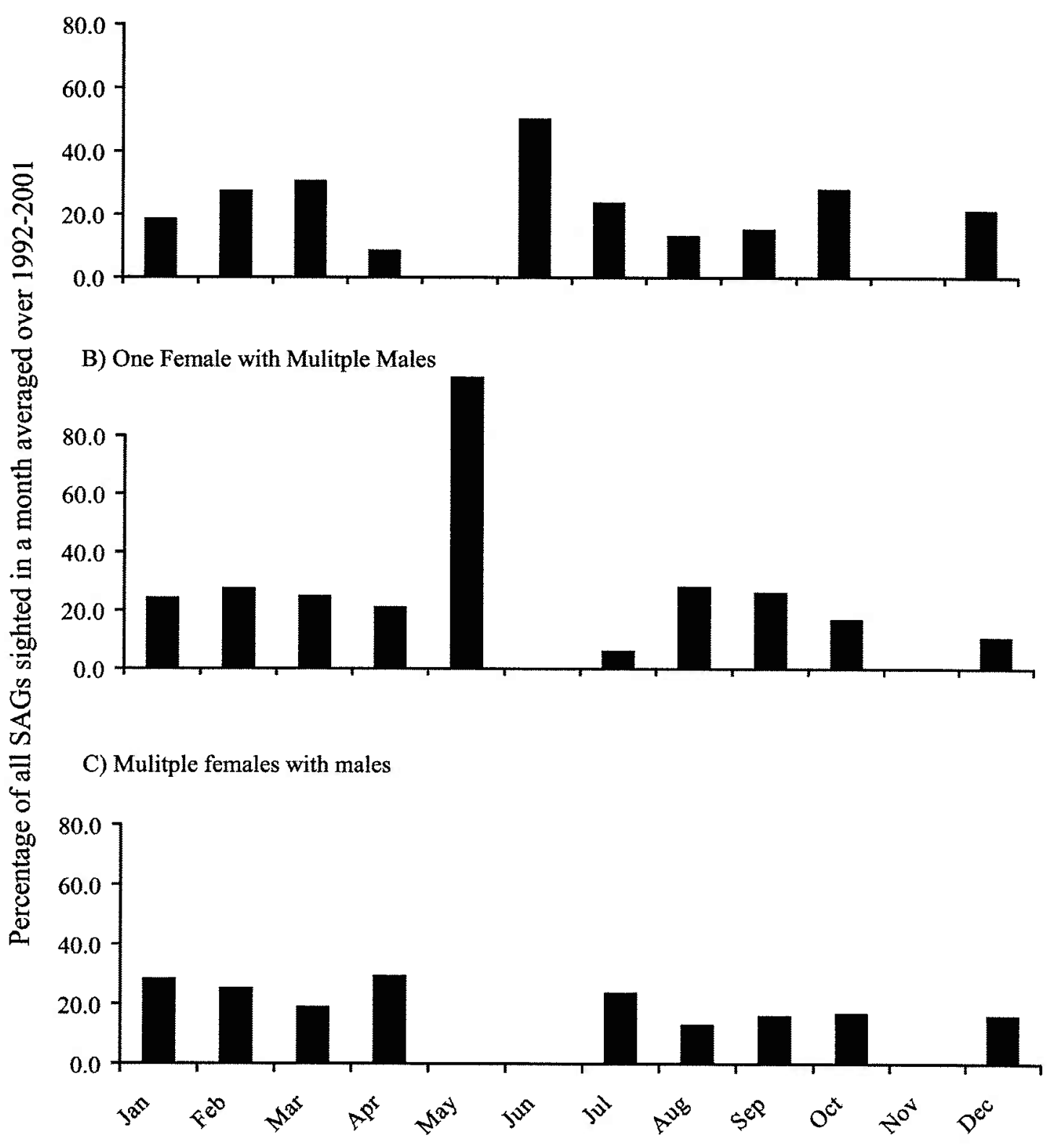

Figure 2.6. Plot showing the distribution of potentially reproductive SAGs as a percentage of all SAGs sighted in a given month averaged over ten years. A) Male-female pair. B) One female with multiple males C) Multiple females with males. 


\section{Non-Reproductive Groups}

A) All-Female
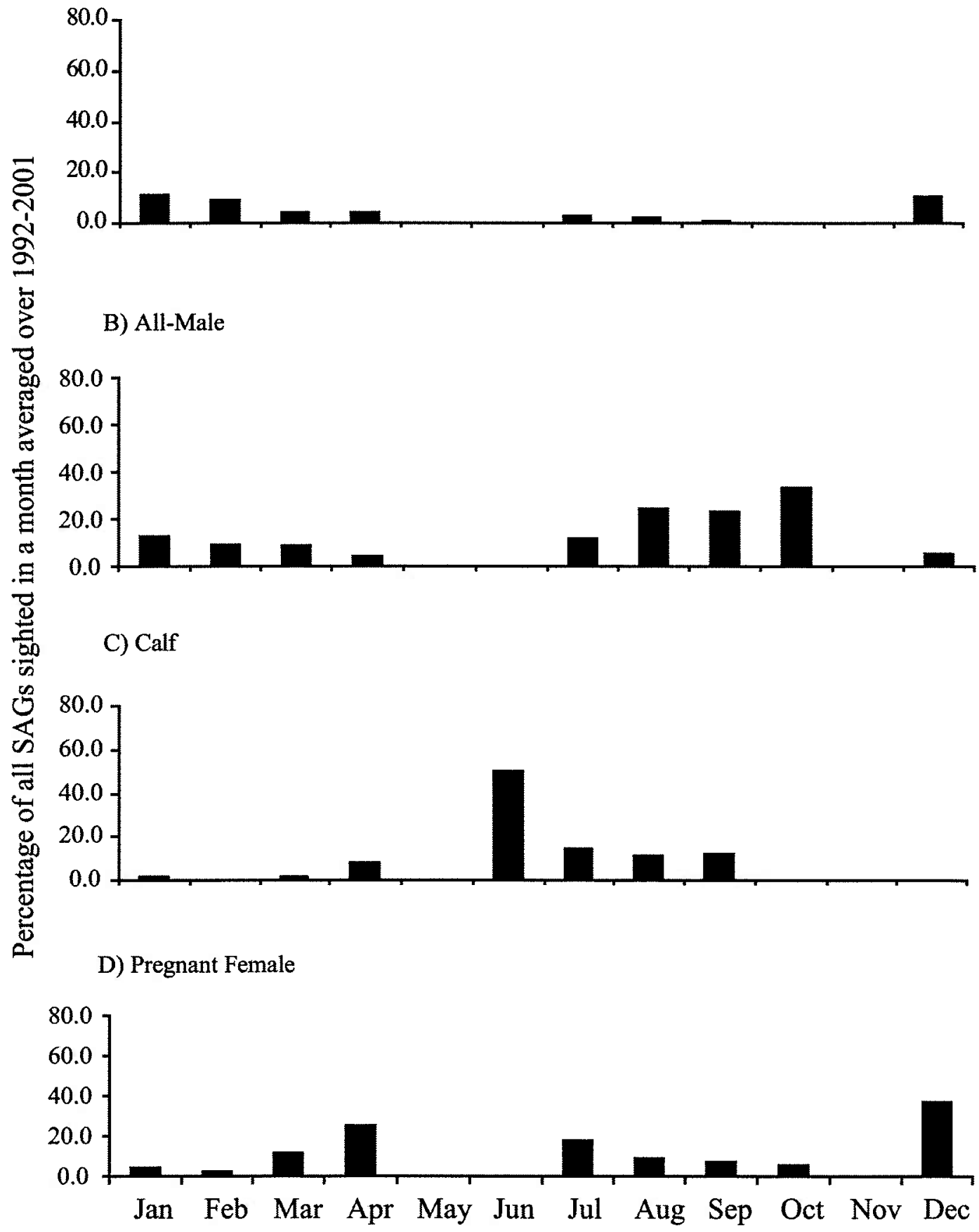

Figure 2.7. Distribution of non-reproductive SAGs as a percentage of all SAGs sighted in given month averaged over ten years. A) All-female B) All-male C) Calf D) Pregnant groups. 
A) South-eastern U.S. $(\mathrm{N}=114)$
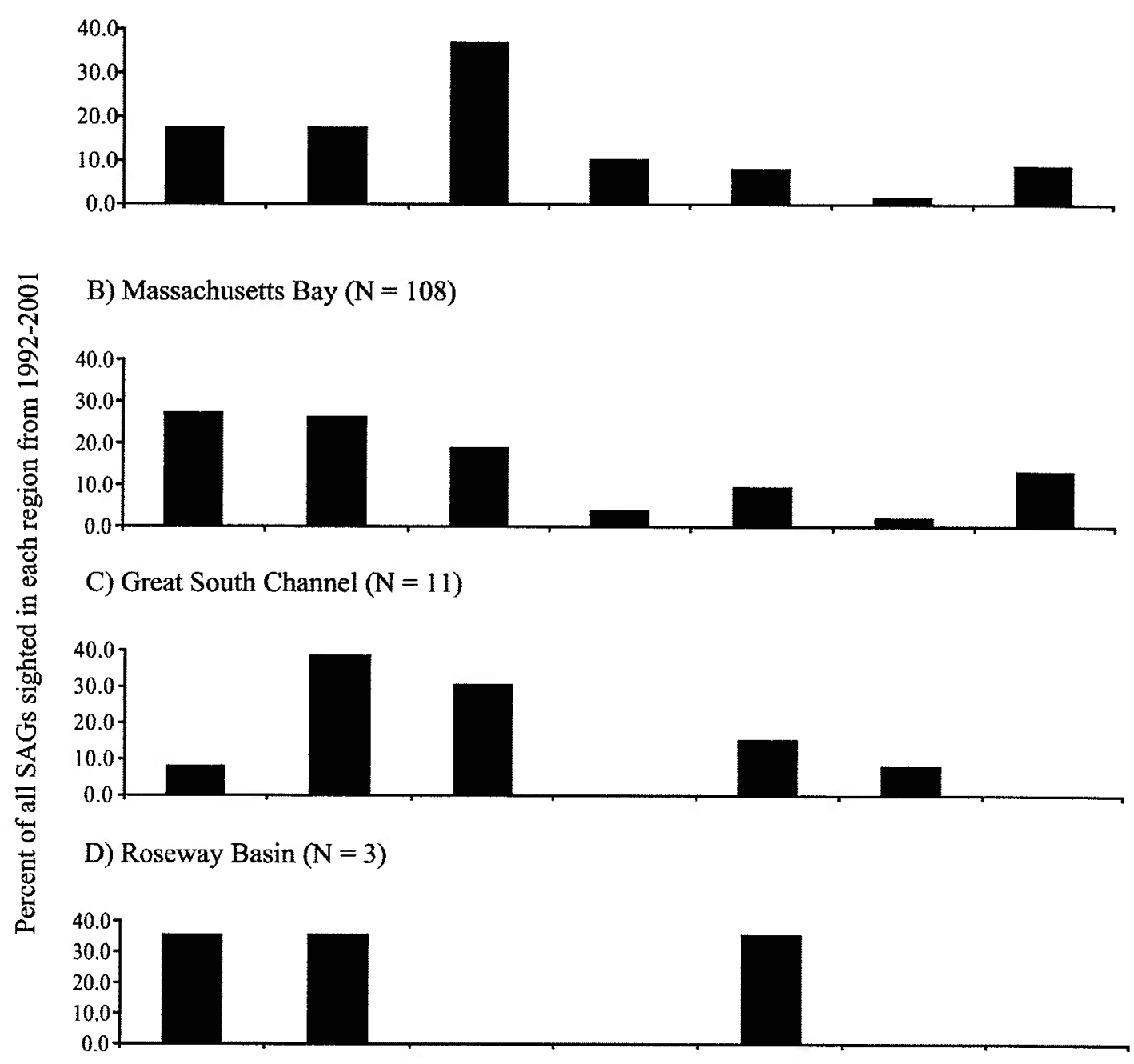

E) Bay of Fundy $(\mathrm{N}=413)$

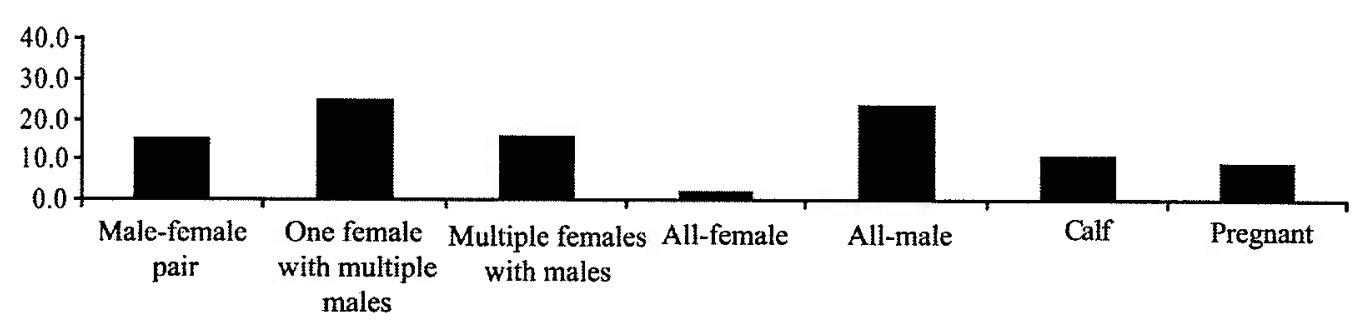

Figure 2.8. The proportion of SAGs of each type sighted in each region between 1992-2001. A) Southeastern U.S. B) Massachusetts Bay, C) Great South Channel, D) Roseway Basin, E) Bay of Fundy. 


\subsubsection{Individual Involvement in SAGs}

The number of sightings of an individual in SAGs ranged from $0-83$ (mean $=$ 14.1, $\mathrm{Std}=13.3, \mathrm{~N}=367$ ). These numbers are dependent on the age and sighting history of each individual. For example, a whale sighted for 20 years may have more SAG sightings than a two year old whale, even if they had the same probability of being in sighted in a SAG. To correct for this, the number of sightings in a SAG was calculated as a percentage of total sightings for each individual. Total sightings were defined as the number of sightings of one individual from its first sighting through $12 / 31 / 2001$. The number of sightings in a SAG was the number of times an individual whale was sighted in a SAG. The percentage of sightings in SAGs ranged from $0-100 \%$ (mean $=25.5 \%$, Std. =17.8). These values include all animals of any age or sex, including those of unknown age and sex. The total number of SAG sightings for all known females range from $0-47($ Mean $=9.7, \mathrm{Std}=9.0, \mathrm{~N}=140)$ and for all known males range from $0-83$ $($ Mean $=20.2, \operatorname{Std}=15.1, \mathrm{~N}=169)$ (Figure 2.9).

a)

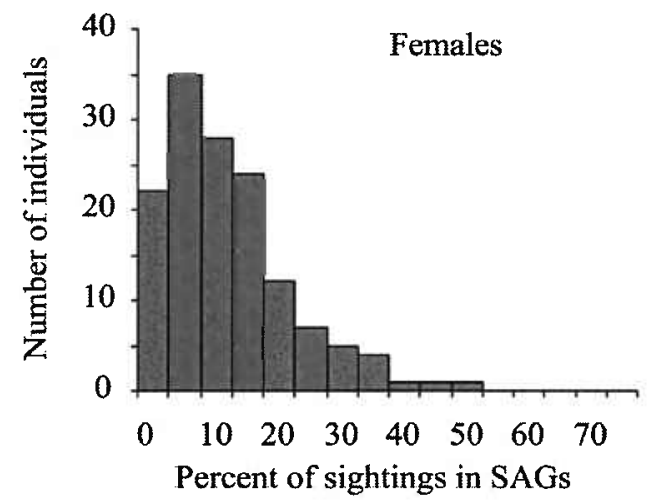

b)

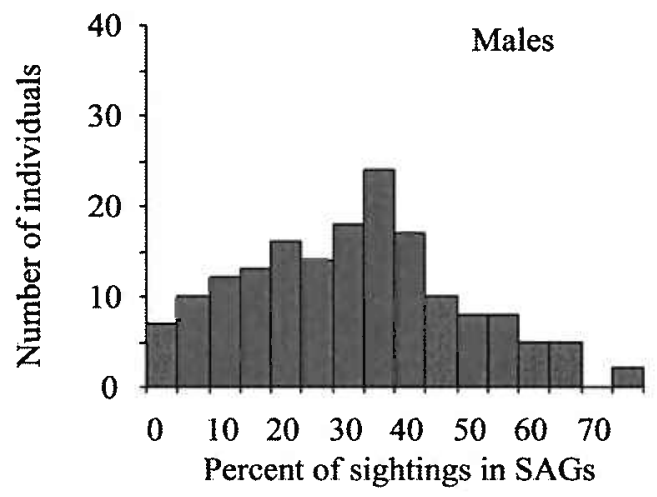

Figure 2.9. Percentage of sightings in $\mathrm{SAG}$ for individual a) female $(\mathrm{N}=140)$ and $\mathrm{b})$ male $(\mathrm{N}=169)$ right whales : 
The proportion of total sightings involved in SAGs for all females range from 0 $50 \%$ (Mean $=14.1 \%, \operatorname{Std}=9.9, \mathrm{~N}=140$ individuals $)$ and for males range from $0-76 \%$ (Mean $=32.9 \% \mathrm{Std}=17.0, \mathrm{~N}=169$ individuals). Males are seen in SAGs for a significantly greater proportion of their sightings than females ( $t$-test, $p<0.0001$ ). Age is known for most individuals born into the population since 1980 and 149 individuals of known age have been sighted in SAGs. The average first age of sighting for whales of known age was 1.5 years $(\mathrm{Std}=1.8)$. Forty-one percent of all whales of known age were seen in SAGs as calves $(\mathrm{N}=62) .90 \%$ of individuals were sighted in SAGs by the time that they were four years old (Figure 2.10). There was no significant difference between males and females in the age of first sighting in a SAG.

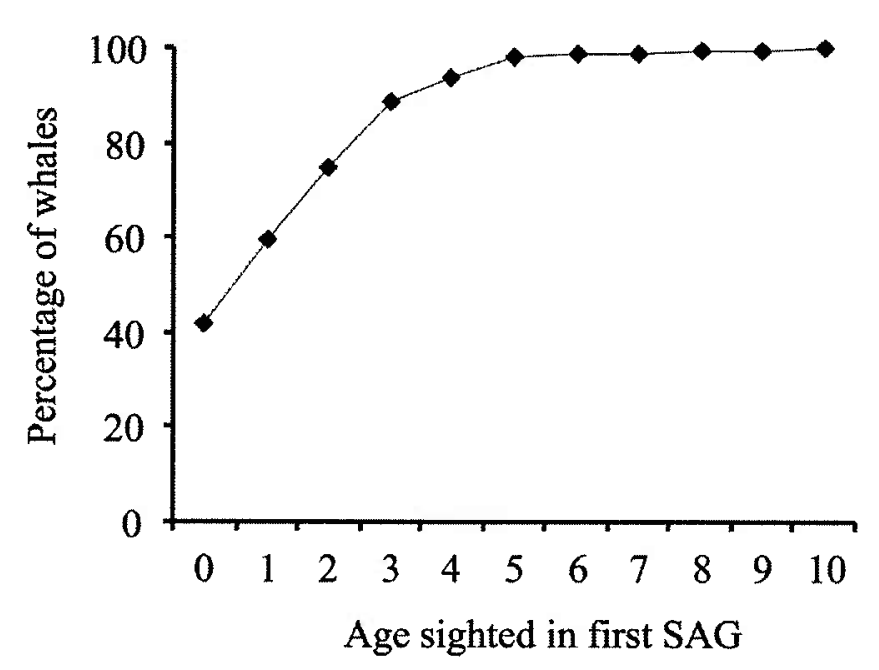

Figure 2.10. A plot of the percentage of known age whales first sighted in SAGs between 0-10 years. $0=$ year of birth.

\subsubsection{SAGs related to calf output}

No correlation was found between the number of SAGs and the number of calves 12-13 months later. This indicates that there is no clear relationship between the observed SAG activity around the time of conception of the calves and the number of calves born. High correlation values were found between the number of SAGs (total number, number of reproductive and number of non-reproductive SAGs) and the number of calves produced 0-11 months later. This indicates that the number of SAGs sighted in the 11 
months prior to the birth of calves is correlated with the number of calves born. The data are presented in Table 2.5. Figure 2.11 shows the values of the total number of SAGs plotted against the number of calves born a) 12-14 months later and b) 0-11 months later.

Table 2.5. Correlation values for the number of SAGs versus the number of calves born 0-11 months later and the number of calves born 12-14 months later. Correlation values are shown for the total number of all SAGs in a given year, the number of only reproductive SAGs (R-SAGs), and the number of nonreproductive SAGs (NR-SAGs). Note that there is a higher correlation value between NR-SAGs than RSAGs.

\begin{tabular}{c|cc}
\hline $\begin{array}{c}\text { Number of } \\
\text { SAGs }\end{array}$ & $\begin{array}{c}\text { Calves born 12- } \\
\text { 14 months later }\end{array}$ & $\begin{array}{c}\text { Calves born 0-11 } \\
\text { months later }\end{array}$ \\
\hline $\mathrm{N}$ total & 0.06 & 0.88 \\
R-SAGs only & 0.05 & 0.71 \\
NR-SAGs only & 0.14 & 0.87 \\
\hline
\end{tabular}

a)

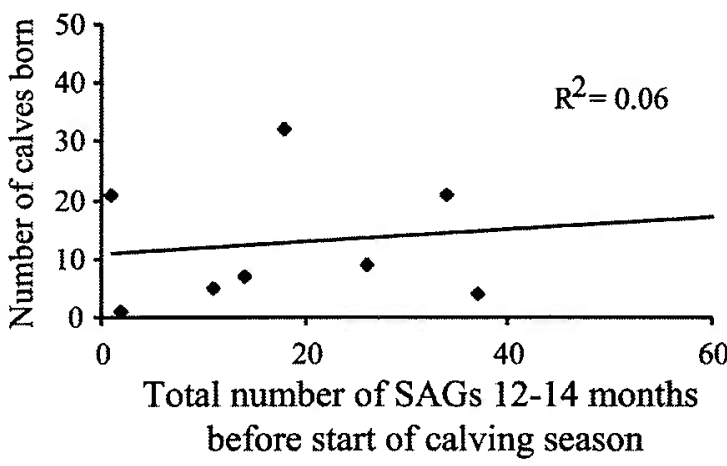

b)

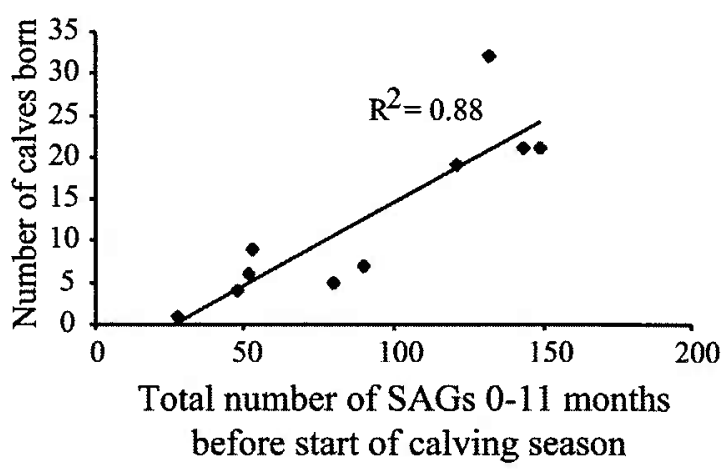

Figure 2.11. A plot of the total number of SAGs sighted a) 12-14 months before the calving season each year and b) 1-11 months before the calving season each year. The line represents the regression line with the $r^{2}$ value listed on each figure.

\subsection{DisCUSSION}

SAGs are commonly sighted during surveys for the North Atlantic right whale in all areas. The behaviors observed in SAGs have led many to suspect that mating occurs in these groups. A summary of occurrence and composition of SAGs in the North Atlantic highlights some of the difficulties in assigning fertilization to all SAGs. These results suggest that there are multiple functions of SAGs that may include, but are not limited to, fertilization in this species. 


\subsubsection{Occurrence of SAGs}

SAGs have been seen in all known habitats of the North Atlantic right whale and in 11 months of the year. The occurrence of SAGs is not consistent with the idea that SAGs function for fertilization. The limited calving period from December to February (Kraus et al., 1986b) suggests that conception is seasonal, unless right whales are somehow capable of delayed implantation of embryos, which allows for a delay between fertilization and gestation. Delayed implantation, while common in pinnipeds, has yet to be documented in any cetacean (Boyd et al., 1999). Therefore the observed patterns of SAG occurrence suggest that SAGs serve multiple functions. Some SAGs likely lead to reproduction. Given the group composition and observed sexual activity in some groups, the sexual nature of some SAG groups, regardless of function in many circumstances cannot be questioned.

Not all sexual behavior is directly linked to reproduction. While most female mammals limit mating to a brief period during the oestrous cycle, females in a few primate species, including humans, remain receptive to mating even when fertilization is not possible (Hrdy, 1981). Several cetacean species have been documented to commonly exhibit non-reproductive sexual behavior and mating (e.g. spinner dolphins (Wells, 1984) and short-finned pilot whales (Globicephala macrohynchus) (Kasuya et al., 1993)). Explanations for this sexual behavior of non-reproductive females typically focus on the use of sexual behavior to strengthen affiliative social bonds between individuals (Hrdy, 1981). While the importance of social bonds in right whales remain unclear (Hamilton, 2002), frequent sexual behavior in SAGs may indicate long-term social affiliations between individuals.

The corrected sightings of SAGs/month (Fig. 2.3 \& 2.4) show a marked increase of SAG activity as the year progresses, peaking in August and September. The apparent decline of SAG activity in October and November reflects a lack of right whale sightings and not necessarily a decline in SAG activity. It has been hypothesized that October and November are the most likely time for whales to congregate and mate based on the 


\section{Chapter 2: Social behavior in right whales}

seasonal calving (Kraus et al., 1986b) and the estimated gestation length (Best, 1994). Researchers often refer to the "missing breeding grounds" for this population as right whales are missing from current survey areas at the expected peak of conception. If the increase in activity seen in August and September were to continue into October and November, then there could be an annual cycle of SAG activity with a peak in October, with the lowest levels seen in May and June. This would be consistent with the expected timing of fertilization.

There may be a protracted breeding season in this species. Relatively high levels of SAG occurrence by month continue beyond the suspected breeding season and through the winter months. SAG occurrence only starts to drop at the end of the calving season. Two hypotheses are consistent with this observation. The first is that females in some mammalian species are induced ovulators (Bronson 1989). It is possible that $\mathrm{SAG}$ behavior is required for female right whales to ovulate. This would explain the high level of SAG activity throughout the year and the increase in frequency around the predicted breeding season. The second hypothesis is that sexual behavior in right whales may involve more than just fertilization. Studies of reproductive behavior in many different species identify several stages and behaviors associated with obtaining mates. The best example for this can be found in passerine bird species. For many species, song can be heard throughout the year, with a distinct peak in song production shortly before the laying of a clutch. The increase in song activity often takes place when males arrive at the breeding grounds before females arrive (Catchpole \& Slater 1995). The males' behaviors are still considered to be associated with reproduction, even if the behavior doesn't lead immediately to fertilization of eggs. These behaviors, involving territory acquisition and establishment of dominance are arguably as important as later displays directed to receptive females. Male behavior on the breeding grounds of humpback whales is thought to follow a similar pattern, with males arriving early on the grounds and beginning to sing before most potentially estrous females arrive (Clapham, 1996). Male humpback whales are also seen competing for access to females in competitive groups (Tyack \& Whitehead, 1983). Female choice and selection of a mate may also play 
a role in an extended breeding season. SAGs in right whales may represent a situation in which males compete to impress female right whales. The rise in male-male groups in the late summer months supports the idea that if male-male interactions may be important for dominance sorting within the population.

SAG behavior with females may represent female sampling of males for later selection of a mate. Chapter 3 indicates that although females produce most of the sounds in SAGs males do make some sounds. Females may be using the sounds to identify individuals. However, if acoustic displays were important for recognition, males would be expected to make more sounds in SAGs. Other potential mechanisms for recognition include vision and chemical cues.

\subsubsection{Group Size and Composition}

It is difficult to argue that the trends in SAG activity reflect seasonal changes in reproductive activity when it is clear that not all SAGs can function for fertilization. The group composition of different SAGs makes it clear that there are distinct group types that can be categorized into potentially reproductive or non-reproductive groups.

The long-term records of identified individuals in this population make it feasible to determine group composition in SAGs related to age and sex of individuals. All SAG sightings were classified into one of seven different types of groups (Table 2.1) ${ }^{1}$. These seven groups can be divided into potentially reproductive and non-reproductive groups. Male-female pairs, one female with multiple males, and multiple females with multiple males are all potentially reproductive groups based on the genders of animals involved. Groups with calves present may or may not be reproductive, depending on whether reproductively receptive females are also present in the groups. All-female, all-male and pregnant female groups are most likely not reproductive in any case, even though these groups often appear to be sexual in nature and may be related to later reproductive events.

\footnotetext{
${ }^{1}$ Groups with unidentified whales known to be involved were not considered in this analysis.
} 
The potentially reproductive groups make up almost $60 \%$ of all SAGs sighted, and they are seen throughout the year (Figure 2.6). The proportion of potentially reproductive groups remains almost constant across regions, with a slight drop in the Fundy region, due to a large increase in the number of SAGs with calves in that habitat. Approximately $40 \%$ of all SAGs involve animals that cannot be mating for conception for one reason or another (e.g. wrong gender, already pregnant, or nursing a calf). The non-reproductive groups pose a challenge to the hypothesis that SAGs function solely for fertilization.

SAG is a broad definition that encompasses several different types of groups. Many non-reproductive group types could be linked indirectly to reproduction, either by dominance sorting in all male groups, or by calves and juveniles learning about SAG behavior from experienced adults. However, two group types, all-female groups and groups with pregnant females as the focal, are difficult to explain in terms of reproduction unless they are a result of hormonal levels of the females involved.

The non-reproductive groups show more seasonal variation than the potentially reproductive groups. All-female groups are most commonly observed in the winter months from December-February. The majority of these groups are seen on the calving grounds off of the Southeastern U.S. The involvement of females in groups on the calving ground may indicate cooperation among females associated with calving or some other social function. Many of the groups with pregnant females are also seen in the Southeast and these groups often are made up of only female whales.

Groups with pregnant females are seen throughout the year. There are two notable peaks, in December just prior to calving, and in March and April at the end of the calving season. Why pregnant females should be involved in SAGs at all remains puzzling. Presumably female whales are generally capable of avoiding unwanted male attention and there seems to be inherent risk in being in close body contact with multiple $50+$ ton animals let alone mating when pregnant. Additional risks include the transmission of disease (Jennions \& Petrie, 2000). In humans and in several primate species, an increase of female receptivity has been documented during the end of the first trimester of 
pregnancy (Hrdy \& Whitten, 1987). This behavior is thought to be a result of hormonal changes due to pregnancy and may help strengthen bonds between mates (Hrdy, 1981). The peak of pregnant females in SAGs in March and April would correspond to the presumed end of the first trimester of a right whale pregnancy. Further research in the hormonal cycles of reproductively active right whales (currently in progress (Rolland et al., 2002)) may provide an explanation for the sexual activity of pregnant females if hormone levels peak in March and April. Short-finned pilot whale females were found to be sexual active even when pregnant, so this behavior in right whales does have a precedent in cetaceans (Kasuya et al., 1993).

It has been suggested that Roseway Basin functions as a mating ground for this population (Hamilton, 2002). Potentially reproductive SAGs would be expected to be the predominant social interaction on the mating grounds. Only all-male groups and potentially reproductive groups have been sighted on Roseway Basin in the past ten years. If all-male groups are connected to reproduction, in terms of male-male competition for mates for example, then the composition of group types seen on Roseway Basin supports this theory.

There is a marked increase in the percentage of all-male groups through the summer into the fall, peaking just before November, when conception would be expected to peak (Figure 2.7). There are several potential explanations for this observation. The all-male groups may be important in determination of male social status through competition with other males in these groups. Alternatively, the behavior may merely represent practice of SAG behavior without females present. Males may also use all-male groups to strengthen bonds allowing males to cooperate in achieving intromission in SAGs involving reproductive females by working together (Kraus \& Hatch, 2001; Payne, 1995). In the humpback whale, pairs of males have been seen entering and possibly cooperating in competitive groups (Clapham et al., 1992). Research into the hormone levels of males throughout the year may shed light on whether there are physiological indications that male reproductive activity peaks in the late fall. 
The involvement of calves in SAGs is very common, but varies depending upon the age of calves. In the Southern right whale, during the first few months after birth, the calf tends to remain very close to the mother, rarely separating more than a few meters (Taber \& Thomas, 1982.). In the North Atlantic right whale there are only four observations of calves in SAGs before 6 months of age. It appears that the mothers choose to keep very young calves out of SAGs until their first summer on the feeding ground as the behavior of the calf is not independent of the mother's behavior during the first few months. The prevalence of SAGs with calves increases and peaks in the summer months and the mother is often present in these groups. This may indicate some functional role for socialization with mothers actively forming SAGs with their calves present, possibly to interact with other whales. Increasing participation in SAGs by the calf in the summer may also be a function of increased independence and curiosity at this age, thought to be shortly before weaning for most individuals (Kraus et al., 2001).

\subsubsection{Individual Involvement in SAGs}

Looking at individual involvement adds more detail to our understanding of SAGs. There are clear differences between males and females in SAG participation, with males seen on average in twice as many SAGs as females. Right whales typically participate in their first SAGs when they are very young. For all animals that have been born over the past 23 years, $41 \%$ were seen in SAGs as calves. Over $90 \%$ were seen in SAGs by their $4^{\text {th }}$ year. There is no evidence for any right whale, male or female becoming reproductively mature before 5 years (Kraus et al., 2001); therefore almost all whales of known age have been seen in SAGs before they are reproductively mature. Juveniles and calves may benefit from practice or experience in SAGs. The second female in multiple female groups in the Bay of Fundy was commonly a juvenile and it has been previously suggested that this allows young females to learn SAG behavior from experienced females (Kraus \& Hatch, 2001). 


\subsubsection{Calving}

The estimated gestation period for Southern right whales is $12-13$ months (Best 1994). Therefore SAGs in the 11 months prior to calving should not be involved in the conception of the calves born. Therefore, the correlation between number of SAGs seen in the 11 months before the calves are born and the number of calves is puzzling. In general the level of SAG activity by year seems to echo the calving productivity stochasticity year to year. Typically there were a high number of SAG sightings the year before a year with high calf production, low SAG sightings the year before years with low calf production (i.e. 1999 with 29 SAGs and 2000 with 1 calf vs. 2000 with 135 SAGs and 2001 with 30 calves) and a high correlation value (.88) for this comparison. This could be explained by the general health status of the population in the years before high or low numbers of calves. Data from other researchers indicate that the health of the population was poor in 1998 and 1999 when the body fat reserves of individuals were low (Miller et al., 2001; Pettis et al., 2001). In years when the fat reserves seem to be building up and health improving, the number of SAGs and the number of calves increase. Another possibility is that surveys are not covering the breeding grounds of this population at the time of conception, and the SAGs related to fertilization are being missed.

Whether or not all SAGs are for reproduction, participation in SAGs must have a cost in terms of valuable energy needed for survival in starvation conditions. Even if direct interaction in a SAG at the surface is a low energy behavior, the time lost for feeding is a clear energetic cost. It is understandable then that there would be dips in both the percentage of sightings of right whales that were SAGs and the total number of SAGs in years when food is scarce and individuals' energy reserves were low. In these years, the costs of the energy expenditure and lost foraging time from SAG participation may be greater than the benefits.

\subsubsection{Conclusions and recommendations for further research}


This analysis of SAG composition and occurrence raises many more questions for further research. Current research in two areas in particular, hormone levels and genetics, will undoubtedly further increase our understanding of the functional significance of SAGs. Hormone research can reveal seasonal trends in hormone levels from animals in different reproductive classes. Hormone levels in three groups in particular, estrous females, pregnant females and reproductive males will be most helpful in explaining seasonal trends in SAG occurrence.

Genetic studies of relatedness between individuals may also make a large contribution in distinguishing between reproductive and non-reproductive SAGs. To avoid inbreeding, males and females in reproductive groups would be expected to be less related than animals in non-reproductive groups. If the relatedness between males involved in reproductive SAGs is greater than the average relatedness of the population it may indicate male-male cooperation via kin selection in these groups.

Non-reproductive social groups may consist of closely related individuals called together by a single female. This would indicate that there may be a matriarchal social system in right whales. If the calls of whales are individually distinctive, it is possible that involvement in these non-reproductive social groups play an important role in teaching whales to distinguish between kin and non-kin. These groups represent times when individuals have an increased opportunity to learn the sounds of closely related females.

This survey supports the original hypothesis that SAGs are likely important both for reproduction and social interactions between individuals. The observed increase in potentially reproductive SAG activity through the summer and early fall corresponds well with the likely peak of conception. The frequency of observation of non-reproductive SAGs throughout the year, particularly the timing of SAG involvement by calves in their first summer, suggest that SAGs also play a role in social interactions between individuals not directly related to fertilization. Further research into the biology of this species may more conclusively demonstrate the biological roles of SAGs in the North Atlantic right whale. 


\subsection{Literature Cited}

Aguilar, A. 1986. A review of old Basque whaling and its effect on the right whale (Eubalaena glacialis) of the North Atlantic. In: Right Whales: Past and Present status, Special Issue No. 10, (Ed. by Jr., R. L. B., Best, P. B. \& Prescott, J. H.), pp. 191-200. Cambridge, England: International Whaling Commission.

Best, P. B. 1994. Seasonality of reproduction and the length of gestation in southern right whales Eubalaena australis. Journal of Zoology, London, 232, 175-189.

Boyd, I. L., Lockyer, C. \& Marsh, H. D. 1999. Reproduction in marine mammals. In: Biology of Marine Mammals (Ed. by Reynolds III, J. E. \& Rommel, S. A.), pp. 218-286. Washington: Smithsonian Institution Press.

Brownell, R. L. J., Best, P. B. \& Prescott, J. H. 1986. Introduction. In: Right whales: past and present status (Ed. by Brownell Jr., R. L., Best, P. B. \& Prescott, J. H.): International Whaling Commission.

Brownell, R. L. J. \& Ralls, K. 1986. Potential for sperm competition in baleen whales. Rep. Int. Whal. Commn. (Special Issue 8), 97-112.

Call, J., Aureli, F. \& De Waal, F. B. M. 1999. Reconciliation patterns among stumptailed macaques: A multivariate approach. Animal Behaviour, 58, 165-172.

Caswell, H., Fujiwara, M. \& Brault, S. 1999. Declining survival probability threatens North Atlantic right whale. Proceedings of the National Academy of Science, 96, 3308-3313.

Clapham, P. J. 1996. The social and reproductive biology of humpback whales: An ecological perspective. Mammal Review, 26, 27-49.

Clapham, P. J., Palsbøll, P. J., Mattila, D. K. \& Vasquez, O. 1992. Composition and dynamics of humpback whale competitive groups in the West Indies. Behaviour, $122,182-194$.

Connor, R. C., Wells, R. S., Mann, J. \& Read, A. J. 2000. The bottlenose dolphin: Social relationships in a fission-fusion society. In: Cetacean societies. Field studies of dolphins and whales (Ed. by Mann, J., Connor, R. C., Tyack, P. L. \& Whitehead, H.), pp. 92-126. Chicago: The University of Chicago Press.

Hamilton, P. K. 2002. Associations among North Atlantic right whales. M.S. Thesis. University of Massachusetts Boston, Boston. 
Hamilton, P. K. \& Martin, S. M. 1999. A catalog of identified right whales from the Western North Atlantic: 1935 to 1997. pp. 27 pp +382 plates. Boston: New England Aquarium.

Harcourt, A. H., Harvey, P. H., Larson, S. G. \& Short, R. V. 1981. Testis weight, body weight and breeding systems in primates. Nature, 293, 55-57.

Hrdy, S. B. 1981. The woman that never evolved. Cambridge, MA: Harvard University Press.

Hrdy, S. B. \& Whitten, P. L. 1987. Patterning of sexual activity. In: Primate Societies (Ed. by Smuts, B. B., Cheney, D. L., Seyfarth, R. M., Wrangham, R. W. \& Struhsaker, T. T.), pp. 370-384. Chicago: The University of Chicago Press.

Jennions, M. D. \& Petrie, M. 2000. Why do females mate multiply? A review of genetic benefits. Biological Review, 75, 21-64.

Kasuya, T. H., Marsh, H. \& Amino, A. 1993. Non-reproductive mating in short-finned pilot whales. Report of the International Whaling Commission (Special Issue) 14, 425-437.

Kenagy, G. J. \& Trombulak, S. C. 1986. Size and function of mammalian testes in relation to body size. Journal of Mammalogy, 67, 1-22.

Kishino, H. \& Kasamatsu, F. 1987. Comparison of the closing and passive mode procedures used in sighting surveys. Rep. Int. Whal. Commn., 37, 253-258.

Knowlton, A. R. \& Kraus, S. D. 2001. Mortality and serious injury of northern right whales (Eubalaena glacialis) in the western North Atlantic. Journal of Cetacean Research Management (Special Issue) 2, 193-208.

Kraus, S. D., Hamilton, P. K., Kenney, R. D., Knowlton, A. R. \& Slay, C. K. 2001. Reproduction parameters of the North Atlantic right whale. The Journal of Cetacean Research and Management. (Special issue) 2, 231-236.

Kraus, S. D. \& Hatch, J. J. 2001, Mating strategies in the North Atlantic right whale (Eubalaena glacialis). The Journal of Cetacean Research and Management (Special issue) 2, 237-244.

Kraus, S. D., Moore, K. E., Price, C. A., Crone, M. J., Watkins, W. A., Winn, H. E. \& Prescott, J. H. 1986a. The use of photographs to identify individual North Atlantic right whales (Eubalaena glacialis). Report of the International Whaling Commission (Special Issue) 10, 145-151. 
Kraus, S. D., Prescott, J. H., Knowlton, A. R. \& Stone, G. S. 1986b. Migration and calving of right whales (Eubalaena glacialis) in the western North Atlantic. Report of the International Whaling Commission (Special Issue) 10, 139-144.

Miller, C. A., Reeb, D., Best, P. B. \& Moore, M. J. 2001. Blubber thickness and reproductive success in right whales. In: Fourteenth Biennial conference on the biology of marine mammals. Vancouver, Canada.

Møller, A. P. 1989. Ejaculate quality, testes size and sperm production in mammals. Functional Ecology, 3, 91-96.

Møller, A. P. 1998. Sperm competition and sexual selection. In: Sperm competition and sexual selection (Ed. by Birkhead, T. R. \& Møller, A. P.), pp. 55-90. New York: Academic Press.

Møller, A. P. \& Ninni, P. 1998. Sperm competition and sexual selection: a meta-analysis of paternity studies in birds. Behavioral Ecology and Sociobiology, 43, 345-358.

Nunn, C. L., Gittleman, J. L. \& Antonovics, J. 2000. Promiscuity and the primate immune system. Science, 290, 1168-1170.

Omura, H., Ohsumi, S., Nemoto, T., Nasu, K. \& Kasuya, T. 1969. Black right whales in the North Pacific. Scientific Reports of the Whales Research Institute, Tokyo, 21, $1-78$.

Payne, R. 1995. Among whales. New York: Scribner.

Payne, R. S. \& Dorsey, E. M. 1983. Sexual dimorphism and aggressive use of callosities in right whale (Eubalaena australis). In: Communication and behavior of whales. (Ed. by Payne, R.), pp. 295-329. Boulder, CO: Westview Press.

Pettis, H. M., Hamilton, P. K., Kraus, S. D. \& Rolland, R. M. 2001. A new technique for visually assessing right whale (Eubalaena glacialis) body condition. In: Fourteenth Biennial conference on the biology of marine mammals. Vancouver, Canada.

Rolland, R. M., Hunt, K. E., Wasser, S. K. \& Kraus, S. D. 2002. Reproductive and stress hormones in right whales. In: 2002 North Atlantic right whale consortium meeting (Ed. by M.Marx). New Bedford, MA.

Taber, S. \& Thomas, P. 1982. Calf development and mother-calf spatial relationships in southern right whales. Animal Behaviour, 30, 1072-1083. 
Tyack, P. \& Whitehead, H. 1983. Male competition in large groups of wintering humpback whales. Behaviour, 83, 132-154.

Wells, R. S. 1984. Reproductive behavior and hormonal correlates in Hawaiian Spinner dolphins, Stenella longirostris. In: Reproduction in Whales, Dolphins, and Porpoises (Ed. by Perrin, W. F., Brownell Jr., R. L. \& DeMaster, D. P.), pp. 465472: Reports of the International Whaling Commission Special Issue 6. 
Chapter 2: Social behavior in right whales 


\section{CHAPTER 3: ACOUSTIC ACTIVITY OF NORTH ATLANTIC RIGHT WHALES (Eubalaena glacialis) IN SURFACE ACTIVE GROUPS}

\subsection{INTRODUCTION}

Little is known about the acoustic behavior of the North Atlantic right whale (Eubalaena glacialis) despite hundreds of years of interaction with humans, first as the "right" whale to kill, and later as the "right" whale to protect from extinction. The major focus of recent North Atlantic right whale research has been on conservation, leaving many basic questions about behavior and sound production unanswered. The notable exception is work done on behavior in the predominant visible social interaction in this population, the surface active group (SAG) (Kraus \& Hatch, 2001). For the research presented in this chapter, extensive recordings were made from right whale SAGs in the Bay of Fundy from 1999-2002 in an attempt to produce a comprehensive description of sounds associated with these groups. The goals of the chapter are to 1) describe the types of sounds recorded and how they compare to sounds described from previous acoustic studies of North Atlantic (Wright, 2001; Matthews et al., 2001; Vanderlaan et al., 2000) and Southern right whales (Eubalaena australis) (Clark, 1982; Clark, 1983); 2) determine the rate of production of the two major sound types, Screams and Gunshots during the SAG and how the rate of production is related to behavior and group composition; 3 ) identify individuals producing different sound types in the groups to determine if there are age/sex class differences in production of sounds; 4) determine the potential for differentiating between individuals based on distinctiveness of their calls; 5) measure the source level of these signals and the ambient noise to estimate the effective range of communication in these groups and 6) consider the potential effects of masking by vessel noise. 


\subsubsection{Right whale acoustics}

Although the sounds made by most baleen whale species have been recorded in the past 40 years, relatively few studies have attempted to link sound production with behavioral observations. The best studied baleen whale species for relating sound production to behavior is the humpback whale (Megaptera novaeangliae). Various studies have demonstrated differences in sound production in different behavioral contexts such as the feeding calls in Alaska (D'Vincent et al., 1985) versus song and competitive group sounds on the breeding grounds (Payne \& McVay, 1971; Tyack \& Whitehead, 1983; Silber, 1986; Helweg et al., 1992). Researchers have also found gender differences in sound production. For example, only male humpbacks sing (Winn \& Winn, 1978).

The Southern right whale remains the second best described species in terms of acoustic behavior. Several studies described sound production in this species (Payne \& Payne, 1971; Cummings, 1972; Saayman \& Tayler, 1973; Clark, 1982; Clark, 1983; Clark, 1984). Additional playback experiments demonstrated the right whale's ability to discriminate the sounds of right whales from those of other whale species (Clark \& Clark, 1980). Much of these data are the result of studies by Christopher W. Clark in the late 70 's that described the repertoire of the Southern right whale and linked the production of particular sounds to behavioral state as the result of extensive observations and recordings between 1976 and 1978 in Patagonia.

Surprisingly, the acoustic behavior of the North Atlantic right whale, located just off the east coast of the United States (incidentally where many of the Southern right whale researchers lived) remained relatively poorly described. Some of the earliest reports of North Atlantic right whale sound production describe low frequency signals of feeding right whales (Schevill et al., 1962; Schevill \& Watkins, 1962). These publications gave a few examples of sounds with little information about the behavior accompanying the sound production.

There has been a recent resurgence of interest in North Atlantic right whale acoustics. The North Atlantic right whale is a highly endangered species, subject to 
anthropogenic mortality from vessel collision and entanglement in fishing gear (Knowlton \& Kraus, 2001). Passive acoustic localization has been proposed to detect right whale presence in regions where they are under the greatest threat (e.g. busy shipping lanes and fishing areas with large amounts of fixed fishing gear) (IFAW, 2001). A number of different organizations have attempted to characterize the call rate, frequency range and source level for the North Atlantic right whale (Matthews et al., 2001; Clark, 1999; Vanderlaan et al., 2000). These data are necessary to determine the feasibility of using passive acoustic localization to detect right whales in areas where they are at the greatest risk from human activities. One attempt has been made to describe the acoustic repertoire of the North Atlantic right whale and to compare it to sounds recorded from the Southern right whale (Wright 2001). Information on the repertoire of the North Atlantic right whale will be critical for effective development of passive acoustic localization systems. Characterization of right whale sounds will allow for discrimination of right whale sounds from those produced by other species. This is essential for the effective development of automatic detection software to monitor array data. Similarly, information on the behavior of North Atlantic right whales related to sound production is important to determine if certain sounds can be correlated with times when right whales are particularly vulnerable (e.g. surface behaviors in shipping lanes, presence of calves at the surface).

\subsubsection{The right whale repertoire: the classification problem}

Marine mammals produce a variety of sounds. A common problem in the study of animal communication involves describing and differentiating different sounds. In many cases, the behavioral context in which sounds were produced is unknown. Attempts to describe the repertoires of different species have typically relied on categorization of sounds into discrete classes based on acoustic characteristics, regardless of the behavioral context. An inherent problem with this method is that sounds that vary in what human observers consider important parameters to discriminate between sound types (such as duration, or fundamental frequency) may or may not be categorized separately by the 
animals themselves. At this point we have little information for most species on how the animals themselves parse the sounds produced by conspecifics.

The ideal method of distinguishing sound types would be to make extensive recordings in the wild and determine if particular sounds are produced only by certain individuals (male vs. female, adult vs. infant) or in particular behavioral contexts (feeding, mating, fighting). I would argue that classification of sounds in this way, based on individuals producing the sounds and the context in which they are produced, is the most natural way to differentiate classes of sounds (Janik, 1998). It illustrates differences in the way that the animals themselves use the sounds in their daily lives, which is often the point of these studies and attempts at classification in the first place. Unfortunately, these data are difficult, often impossible, to obtain. Even if a complete individual repertoire was determined, it still might not be possible to distinguish graded signals that may convey different meanings based on subtle differences. Targeted studies of particular age/sex classes or behavioral contexts are a useful way to start this kind of study.

There are three main methods used to classify sound types. These have met with varying levels of success and criticism. The traditional way of making these classifications has been for a human observer to listen to sounds or look at spectrograms and determine "types." This method relies on the pattern recognition abilities of the observers. This method has been used in several different studies. Often there is one human observer, the researcher, making the classifications based on extensive experience with recordings from a particular species (Deecke et al., 1999; Janik \& Slater, 1998). Additional studies have given naïve observers sounds or spectrograms to group together based on similarities (Janik, 1999; Deecke et al., 1999; Wright, 2001). Both of these methods are subject to observer bias, as different observers often focus on different aspects of the sounds or spectrograms to base their classifications on. There is often high reliability of these observer judgments but there may be some disagreement between observers when multiple observers are used. This method has been most successful when discrete signal types with high variation between types are produced. 
The second method uses multivariate statistical methods to discriminate between call types based on "unbiased" quantitative measurements of the sounds. The measurements range from a few simple descriptions of the sounds including duration and multiple frequency measurements to large numbers of measurements, such as those calculated in the Acoustat program developed by Kurt Fristrup which takes up to 128 measurements of each sound (Fristrup \& Watkins, 1994). These measurements are then used to group sounds together, either using methods such as principal components analysis (PCA) (e.g. (Clark, 1983), cluster analysis (Wright, 2001), CART or neural networks (Deecke et al., 1999; Potter et al., 1994). These multivariate classification methods are typically successful in segregating out stereotyped calls, but often fail with graded signals, or with signals characterized by rich harmonic energy that is often ignored in the initial measurements of the sounds (Cortopassi \& Bradbury, 2000). Another method using spectrogram cross correlation and principal ordination measurements shows promising results on a training set of harmonically rich sounds from parrots (Cortopassi \& Bradbury, 2000). Classifications based on multivariate methods are more repeatable and consistent than those from naïve observer visual classification of spectrograms, as different researchers will reach the same results when analyzing the same data. However, they are not necessarily free from bias. The researchers select the measurements to make of the sounds, often selecting those that they suspect will be most useful in separating sounds based on their own personal classification.

The third method of classifying sounds involves determining how animals respond to playbacks of sounds. If animals show different behavioral responses to different sounds, that would give some indication of important perceptual differences to the animals. Habituation trials can be used to determine what changes to a sound are perceived as different (Hopp \& Morton, 1998). Different characteristics of calls can be synthetically modified to investigate the impact of gradual changes of signal characteristics, mimicking the differences in some graded types of signals. Habituation trials give added insight into the perceptual abilities of animals including what features of sounds they attend to (Rendall et al., 1996; Cheney \& Seyfarth, 1988). This can be 
helpful in teasing out differences in graded classes of signals. The playback method is also limited in several ways. How natural are the responses of animals to playback experiments? If sounds recorded from the wild are used, how might individual variation (both in the signaler and the receiver) be influencing response? If synthetic sounds are used, how well do they represent the features important to the animals for perception?

Regardless of the method of classification, it is still important to describe the full repertoire of sounds produced by a particular species, even if the individual or the behavioral context is unknown. For example, repertoire analysis can be used for detection of species in environments where they are difficult to detect visually (Clark \& Fristrup, 1997). For marine mammal species, and the North Atlantic right whale in particular, this is very important. Knowing what recorded sounds are from a right whale, as opposed to humpback whale or some local fish species, is crucial to conservation measures currently being developed. Therefore, some attempt at repertoire analysis needs to be made, even if we lack a complete knowledge of the behavior or demographics that accompany this sound production.

Two previous studies of right whale acoustics, Clark (1982) and Wright (2001), have classified right whale sounds into discrete categories. These two studies used a combination of classification techniques, including visual classification by human observers (Clark, 1982; Wright, 2001) and various multivariate techniques (1) principal components analysis (PCA) (Clark 1982), 2) hierarchical cluster analysis (Wright 2001) and 3) discriminant analysis (Wright 2001). The two studies resulted in similar findings for the repertoires of the two species, although their terminology for and total number of discrete classes of sounds differed.

The overall conclusion from both studies was that right whale sounds could be grouped into three discrete classes; Blow sounds, Slaps and Calls (Clark 1982). Within these discrete classes, there were discrete subclasses (in Blows and Slaps) and both a graded continuum and discrete classes of calls types (Calls). Clark described three types of Blow sounds (normal, tonal and growl) and four types of Slaps (flipper, breach, lobtail and underwater) (Clark 1982, 1983). The Calls were characterized by a graded continuum 
with certain types more commonly observed than others. Clark divided the calls into six types; Upcalls, Down calls, Constant calls, High calls, Hybrid calls and Pulsive calls (Clark 1982) (Table 3.1).

Similarly, Wright (2001) found that Blow and Slap categories were easily separated from all other call types. However, her division of the Calls varied from Clark (1982), resulting in seven types; Low contact call, Down call, Constant call, Hook call, Modulated call, Convex call and Concave call (Wright 2001). The Upcall, a highly stereotyped signal recorded in the Southern right whale and described by Clark (1983) as a "contact call" was absent from Wright's (2001) multivariate analyses. The limited sample of calls used in her study ( 246 calls subjectively selected out of 3606 from 18 hours of recording) used for the multivariate analysis and ultimate classification may explain this difference. The Upcall was indicated in the human observer classification results (Wright 2001). Additionally, band-pass filtering of the recorded signal between $150 \mathrm{~Hz}$ and $5 \mathrm{kHz}$ (Wright, 2001) may have made detection of lower frequency calls more difficult. Comparison of the descriptions of the call types in both studies indicate that the calls described by Wright could be broken down into the same categories described by Clark. There was considerable overlap in the description of call types. This suggests that, generally speaking, there may not be any significant difference in the overall repertoires between the species. 
Table 3.1. Table from Clark (1983) showing the eight sound types found in Southern right whale repertoire.

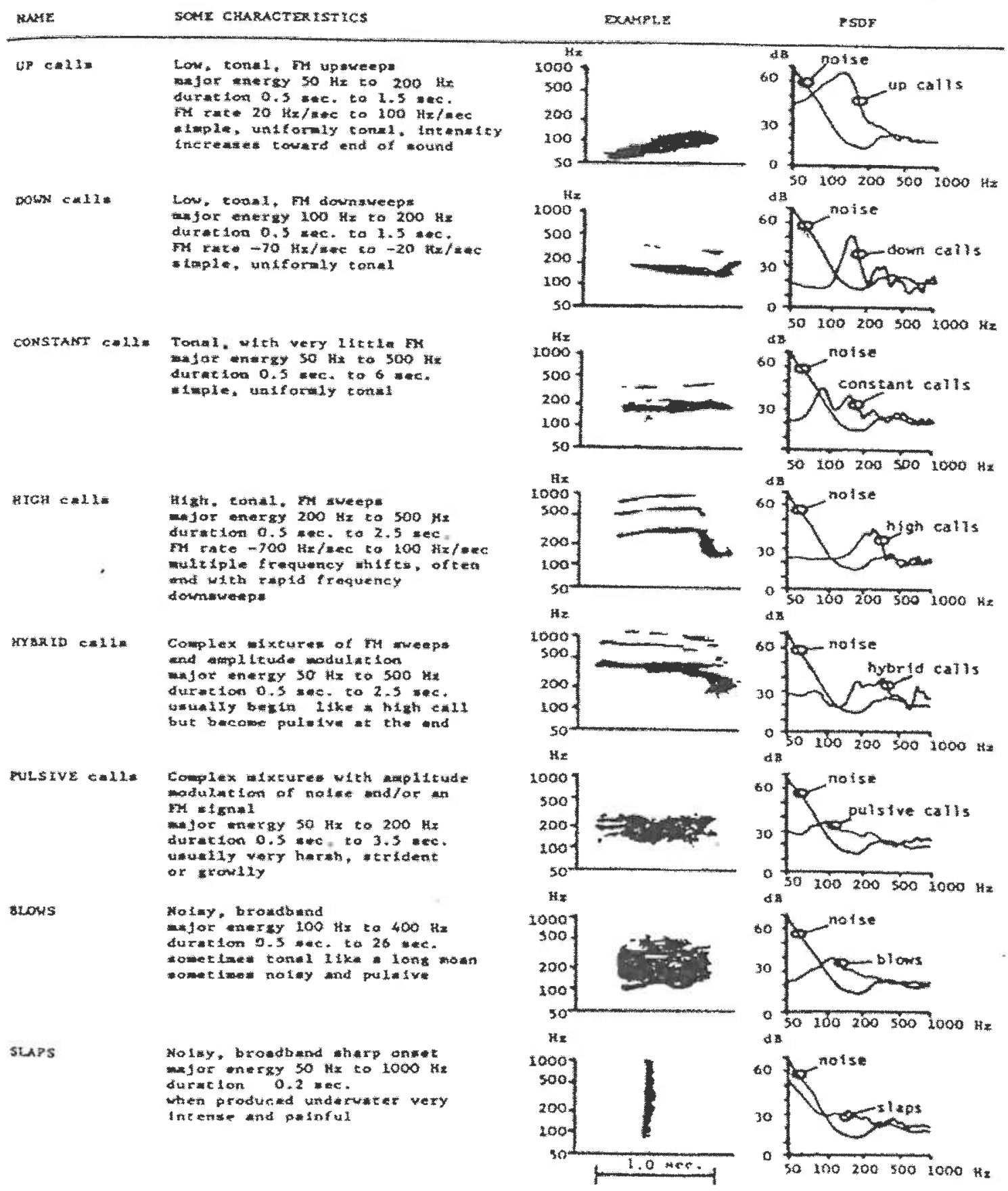




\subsubsection{Sound production related to behavior}

Many studies relating sound production to behavior attempt to correlate behavioral state and sound production (Clark, 1983; Bradbury \& Vehrencamp, 1998). Many studies attempt to do this by obtaining extensive recordings sampled from all age and sex classes in all possible behavioral states. It is often more productive to focus on a single age and sex class. For Southern right whales, Clark carried out these analyses, describing particular classes of calls both by assuming their production was dependent on activity level (Clark 1983) and independently showing the association between call types and particular activity levels (Clark 1982). His studies showed that different call types were associated with different levels of activity. Most notably, Up, Down and Constant calls were associated primarily with swimming or mild activity, while High, Hybrid, and Pulsive calls were the predominant signals produced in fully active and sexually active groups (Clark, 1983).

The North Atlantic right whale is rarely seen close enough to shore to make effective land-based behavioral observations like those used in Southern right whale studies (Clark \& Clark 1980; Clark 1982, 1983). This limits behavioral observations to boats, imposing significant limitations on the quality and duration of sampling. The boat is a potential source of disturbance to the whales and is an inferior platform for recording (due to noise and instability of the platform) when compared to the cliff-based observations and recordings with bottom-mounted hydrophones used by Clark (1983). The inherent limitations of observing undisturbed behavior of whales from a vessel make it difficult to relate sound production to undisturbed behavior. Therefore, this study focused more narrowly on a single behavioral context.

In this study, a distinct, easily detected behavioral context was selected for detailed acoustic characterization. This behavioral context is the SAG of the North Atlantic right whale. A SAG is described as two or more whales interacting at the surface with frequent physical contact with the number of individuals in the group ranging from 2-40 (Chapter 2). This general definition encompasses three types of interaction in 
Southern right whales described by Clark (1983) as mild, full and sexual activity. The composition of the SAG often involves a single female with multiple males (Kraus \& Hatch, 2001). However, SAGs have variable composition, including all-male and allfemale groups, and it is possible that different compositions representing significantly different behavioral interactions have all been lumped under the same label (Chapter 2). Recordings of acoustic activity in these groups may make it possible to distinguish different kinds of interactions in the North Atlantic right whale. Linking differences in acoustic behavior to different group compositions may aid in an attempt to determine the function and significance of these groups to this population. For example, Clark (1983) provides evidence that groups involving sexual activity had different call types than groups of other activity levels. Most notably, the Upcall, was absent from all recordings from groups labeled as sexually active in the Southern right whale (Clark 1983).

The timing of sound production can also be used to differentiate between particular behavioral interactions. A typical measure used to define the rate of sound production is the inter-call interval, defining the time between production of two successive sounds. Sounds are often produced in clusters with rapid sound production, separated by periods of silence. Each cluster of sounds is referred to as a bout, with the time between bouts termed the inter-bout interval (Slater \& Lester, 1982). In this way, call timing within a bout can be considered separately from the inter-bout intervals.

For this study, recorded sounds were classified to compare the sounds produced in SAGs to the overall North Atlantic and Southern right whale repertoires as described by Clark (1983) and Wright (2001). Discrete classes of sound types that were in agreement with previous results were retained. General classes include Slaps, Blow and Calls. For the subsequent analyses, the highly graded signal types in the call group, described by Clark (1983) as High calls, Hybrid calls and Pulsive calls, were lumped together as 'Scream' calls. The rate of call production and the interval between bouts was determined for the two primary classes of sounds, Screams and Gunshot sounds. The source of sound production was investigated using acoustic localization to determine differences in sound production by individual and age or sex. It was sometimes possible to ascribe a call to a 
particular individual but more often the call could only be ascribed to the SAG.

Differences in the Scream calls related to the age of the focal whale were measured. The potential for individual recognition based on variation in the Scream calls were considered for multiple recordings from the same individual. Finally, the source level for different sounds and the noise level in the same frequency band were measured to address the question of effective range of communication in these groups.

\subsection{METHODS}

\subsubsection{Data collection}

\section{Sampling protocol}

Recordings and behavioral observations were made in the Bay of Fundy, Canada, east of Grand Manan Island from 1999-2001 (see figure 4.2 for approximate recording area). The goals of observations in order of importance and feasibility for collection were to 1) obtain acoustic recordings from SAGs and to document group size 2) identify the focal animal 3) identify all members of the group 4) localize sound production in the group 5) measure range to any whale producing sounds to estimate source level. Single hydrophone recordings were made opportunistically from four platforms from 1999-2001 and synchronous hydrophone/video recordings were made when feasible. The four recording platforms were the $\mathrm{R} / \mathrm{V}$ Nereid (1999-2001), a 9m inboard motor vessel owned and operated by the New England Aquarium, the R/V Song of the Whale (2000) a $14 \mathrm{~m}$ motor sailor owned and operated by IFAW, the R/V Bonita (2000-2001) a $5 \mathrm{~m}$ rigid hull inflatable from the New England Aquarium, and the R/V Stellwagen (2001) a $21 \mathrm{~m}$ inboard motor vessel chartered by the Woods Hole Oceanographic Institution. In 2002 a combination of single hydrophone, synchronous hydrophone/video, and 16-channel hydrophone array recordings were made during each observation. A single dedicated platform, the R/V Callisto, a $7 \mathrm{~m}$ outboard from the New England Aquarium, was used in 2002. 
Recordings were made in various sea states, ranging from 0-4 (Beaufort scale), limited by the capacity of the recording vessel to provide a safe recording and observation platform. Most recordings were made in sea states $<3$. The duration of the recordings from 1999-2001 depended on the schedule of the platform. In 2002, recordings were made from the first sighting of the SAG until the end of the SAG or until sea state or light conditions necessitated return to shore. Most SAG observations and recordings were made during daylight hours, with the exception of one recording made at night from the Song of the Whale on $8 / 22 / 00$. Recordings in all years were made with the vessel positioned between 50 and $500 \mathrm{~m}$ from a SAG with the engine shut down for the recordings. For array recordings, attempts were made to position the vessel so that the SAG was directly abeam of the center of the array at a range of $150 \mathrm{~m}$ (Figure 3.1). If the SAG approached the vessel to a range of less than $50 \mathrm{~m}$ or the vessel drifted more than $500 \mathrm{~m}$ from the $\mathrm{SAG}$, recording was suspended and the engine was started to reposition the vessel. Groups were approached to within $40 \mathrm{~m}$ to obtain photographs of whales in the group. When possible, this close approach for photographs was made only once, before recordings began.

\section{Recording Equipment}

\section{Single Hydrophone}

Single hydrophone recordings were made with a Hi-Tech HTI-94-SSQ hydrophone (nominal frequency response $2 \mathrm{~Hz}-30 \mathrm{kHz}$ ) into a TASCAM DA-P1 DAT recorder (nominal frequency response $20 \mathrm{~Hz}-20 \mathrm{kHz}$ ) sampling at $44.1 \mathrm{kHz}$. These hydrophones were used to make synchronized underwater hydrophone recordings and video recordings to couple behavioral and acoustic observations in real time. The video camera used was a Sony DCR TRV-900 mini-DV handheld camera with a nominal frequency response of $10 \mathrm{~Hz}-20 \mathrm{kHz}$, flat from $250 \mathrm{~Hz}-15 \mathrm{kHz}$. Hydrophone depth was shallow and varied from $5 \mathrm{~m}$ for the video hydrophone to $10 \mathrm{~m}$ for the DAT recordings to allow for quick retrieval when whales approached the vessel too closely. 


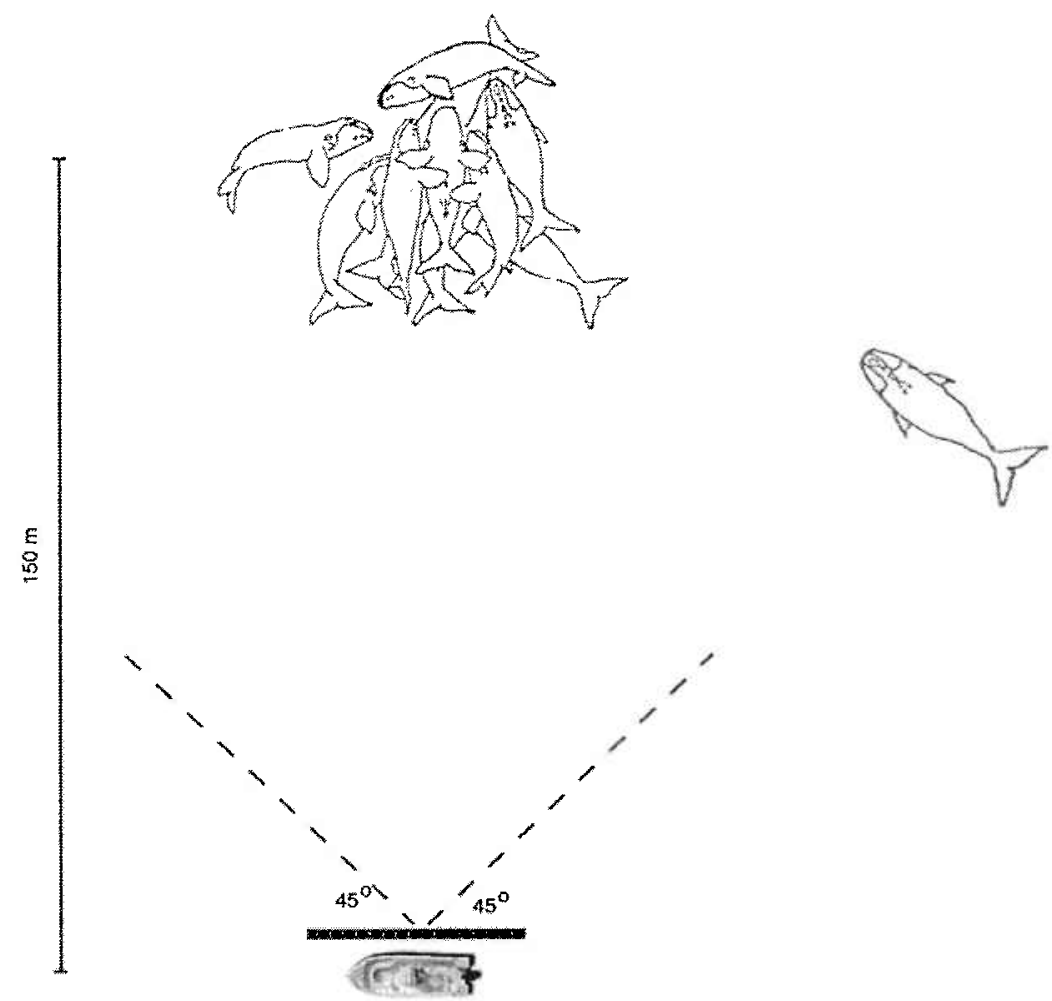

Figure 3.1. Position of the vessel during array recording. The vessel was positioned with the SAG between 150 and $300 \mathrm{~m}$ away, with the whales centered perpendicular to the axis of the array. The surface currents and wind would push the vessel at a rate of .5-3 knots over the surface. The vessel was positioned so that it would drift away from the SAG, preventing the array from being pushed under the boat or the boat drifting too close to the whales. Frequent repositioning was necessary to keep this approximate position for observations. Whales in figure adapted from Kraus \& Hatch (2001).

\section{Hydrophone array}

The recording system consisted of a $3.75 \mathrm{~m}$ rigid linear array of 15 Benthos AQ2TS hydrophones with custom pre-amps with $40 \mathrm{~dB}$ gain. The array design and pre-amps were based on an array design developed for use with killer whales (Orcinus orca) (Miller \& Tyack, 1998). The hydrophones were evenly spaced $0.25 \mathrm{~m}$ apart and mounted on a 1" diameter PVC pipe cut in half length-wise and encased in a $5 \mathrm{~m}$ mineral oil-filled plastic tube. The $0.25 \mathrm{~m}$ spacing corresponds to an upper frequency of $3 \mathrm{kHz}$ to avoid spatial aliasing. The array was manually deployed at $5 \mathrm{~m}$ depth off the side of a stationary $7 \mathrm{~m}$ Mako, the "R/V Callisto" in 2002. The array was buoyed with foam spar buoys made 
of pipe insulation in three positions. This maintained a horizontal position of the array in the water while the vessel was stationary during behavioral observations. This stationary deployment was a modification for this array, which was originally designed to be towed behind a vessel in motion.

The signal from each hydrophone was acquired through a low power $(30 \mathrm{~mW})$, single supply, battery powered, 16 channel, single ended, computer programmable, bandpass filter with adjustable corner frequencies and gain designed by Robert $B$. MacCurdy, an electrical engineer at the Bioacoustic Research Program at Cornell University (CUBRP). The real-time digital data acquisition set up consisted of a National Instruments PCMCIA DAQCard-6062E (12 bit 500kS/s sampling) in a Dell Latitude C610 laptop using the program Chickadee developed at CUBRP. Sounds for all channels were acquired at $8 \mathrm{kHz}$ sampling. The beamforming frequency cut off was $2.5 \mathrm{kHz}$.

Real time beamforming was carried out in the field to determine the bearing to sound sources during behavioral observations. The beamforming software, the CUBRP Matlab Time-Delay Beamformer used acoustic data from 4 hydrophones in the array and was developed by Kathy Dunsmore and Kurt Fristrup at Cornell University. The same software was used for later processing of save files to confirm that observed bearings to whales and the calculated bearings to the sounds agreed.

\section{Behavioral observations}

\section{Photo identification}

Photographs were taken of callosity and scar patterns with a $35 \mathrm{~mm}$ camera (Canon EOS Rebel and 5) using a 100mm-300mm lens. Photographs were taken of each whale present in the groups to determine the age and sex of individuals involved. Photographs were compared to the North Atlantic right whale catalog maintained by the New England Aquarium right whale research group in Boston, MA by staff from the New England Aquarium Right Whale Research Group. 


\section{Video recordings}

Video of SAG behavior was collected during most underwater acoustic recording sessions. Hydrophone signals were fed directly into the video camera when feasible. Additional microphone input into the video camera served as the primary documentation of behavioral observations and observer comments. Behavioral observations included group size, location and behavior of the focal animal, bearing and range to the SAG. Video records of SAGs were used for audio transcription of observer comments, to aid in the identification of individuals with poor quality $35 \mathrm{~mm}$ photographs, and to document the behavior of particular individuals. The behavior of the focal whale during sound production events was analyzed to determine if the focal whale was breathing at the same time that particular sounds were being produced.

\section{Bearing and range measurements}

Bearing (in degrees) and range (in meters) were taken to SAGs and specific individuals separated from the group during 2002 hydrophone array recordings. This allowed comparison of array bearing of whale sounds to the location of individual whales relative to the array. Bearing was taken using a digital $\mathrm{KVH}$ Datascope and range was taken with a Leica LRF 800 laser range finder.

\subsubsection{Data analysis}

\section{Sound Classification}

Sounds recorded from North Atlantic right whales were labeled as one of the following sound types based on similarity to categories described for Southern right whales: Scream (corresponding to High, Hybrid and Pulsive calls (Clark, 1983)), Gunshot (corresponding to Underwater Slap sounds (Clark, 1983)), Noisy Blow, Upcall, 


\section{Chapter 3: Sound production in SAGS}

Down call, and Warble. The decision was made to lump together the High, Hybrid and Pulsive calls under one label to simplify the analysis. These three calls types were all produced at high rates in the recorded SAGs and dominated the whale sounds recorded. Unlike all the other classes of calls, these three call types are not stereotyped. The gradation of the call types, as described by Clark (1982, 1983) and Wright (2001), makes it particularly difficult to separate these three classes of calls by multivariate analyses. Therefore they were lumped together for all further analyses. The additional class of sounds, labeled Warble, consisted of scream-type calls that were faint and quavery. These Warbles were only recorded when calves were present. These Warble calls were labeled based on subjective perception of differences by the author and not based on results from multivariate sound classification nor distinct variation from previously defined call classes.

Acoustic recordings were digitized into .aif files using Cool Edit Pro v1.2 (Syntrillium software). Recordings were acquired at $44.1 \mathrm{kHz}, 16-$ bit sampling rate. Spectrograms of recordings were visually inspected by the author using Matlab 12.0 (Mathworks, Inc.). Each whale sound detected in the recordings was extracted and saved as a separate file using MATLAB browsing software developed by the CUBRP. All extracted sounds were measured using the Acoustat program developed by Kurt Fristrup (Fristrup \& Watkins, 1994). Acoustat was selected for measurements because it contains multiple measures of many variables in each sound cut that were not selected specifically to categorize right whale sounds. In this way I hoped to reduce bias in selection of measurements used for categorization of the sounds. The Acoustat output consists of 128 variables. Of these variables, 20 result from Acoustat settings or are sensitive to the variables of the file that are independent of the sound being analyzed (such as position of the sound within the file or the signal-to-noise ratio of the recording) and were removed before using the measurements for multivariate analyses. The removed variables were CN, LF, HF, Bsize, Xsize, Olap, CS, NumBlocks, ERGtot, ENVmod5, ENVmed5, ENVupp5, ENVmod7, ENVmed7, ENVupp7, Mmnum, SWPnum, ZERnum, ERGmed (See Fristrup \& Watkins 1994 for entire list of 128 variables calculated for each sound). 
Measurements from Acoustat were used to carry out multivariate statistical comparisons of sounds to determine classes. Principal component analysis and cluster analysis (Tabachnick \& Fidell, 1983) were carried out on 100 sounds (20 from each of the major sound types recorded) selected randomly from the recordings to compare classification results to those reported by Clark (1983) and Wright (2001). After confirmation of similar classification results to those previous reported for right whale species, spectrograms of extracted whale sounds were visually inspected and compared to classifications of sound types described in Clark (1983) (Table 3.1). CART (Classification and Regression Trees) analyses were carried out on the entire sample of whale sounds and on a smaller randomly selected subset of whale sounds from all classes to demonstrate the distinctiveness of these classes (Breiman, 1984). Variation in call types was analyzed as a function of group size and composition.

\section{Call timing}

Call timing was measured for the two main classes of whale sounds: Screams and Gunshots. The interval between the start of two successive sounds was used to determine the inter-call interval (ICI). The production of both Screams and Gunshots was characterized by bouts of high sound production separated by long periods of silence. Log-survivorship analysis was carried out for both sound types to determine the bout criterion interval (BCI) (Slater \& Lester, 1982; Martin \& Bateson, 1993; Janik \& Slater, 1998). The average and standard deviation for within-bout and between-bout intervals were calculated using the BCI. The within-bout inter-call intervals and the between bout inter-call intervals were then compared using a student's t-test (Devore, 1995).

These inter-call measurements are strongly influenced by the duration of recording. For example, the longer between-bout intervals were only detectable in longer recording sessions. Longer recording sessions also resulted in more inter-call intervals to measure and represented a large proportion of the total inter-call interval sample.

The measurement of number of calls produced per minute (CPM) was an attempt to standardize measurements between SAG recordings of varying duration. The numbers 
of Screams or Gunshots produced per minute were tallied for all recordings. This method allowed for detection of periodic bouts of very high call rates that are missed by the analyses of within-bout inter-call intervals that are averaged over the entire recording. This contrasts to other methods of calculating call rate that total up the number of sounds and divide by the total time of a recording (Howard \& Young, 1998). The average CPM rate of production was calculated for each sound type and each SAG. The differences in CPM between Screams and Gunshots were compared using the Mann-Whitney Test (Devore, 1995) due to non-normal distribution of the data. Differences in CPM by call type within a SAG were compared to differences in group size and composition.

\section{Individual sound production}

The identification of the whale producing particular sounds can add a great deal to our understanding of interactions between individuals in SAGs. There would be very different predictions for group function if 1) the female produces all the sounds in the group and males are silent 2) males produce all the sounds in the groups and females are silent or 3 ) both males and females produce the sounds in the groups. This section addresses this issue.

\section{Array data}

Data from the hydrophone array were analyzed with the Cornell beamforming software to obtain bearings to all recorded calls. The acoustic bearing to each call was then compared to visual bearings to particular whales. Differences in angle between the bearing to the sound and the observed bearing to whales were calculated. Comparison of acoustic bearings to vessel noise and visual bearings to the known sound source were also made as a control for the accuracy of the array bearings.

\section{Video data}

Video data were analyzed to determine if the focal animal was breathing when Screams or Gunshots were produced. Baleen whales may not be able to generate 
internally produced sounds during exhalation. The precise sound production mechanism is not known for any baleen whale, but no known observations of whales vocalizing during respiration in this and previous studies of right whales (Clark, 1982), is taken as indirect evidence that the focal animal would not be breathing while producing sounds. It is likely that calls cannot be produced simultaneously with an exhalation in right whales because opening the blow holes would result in loss of the pressurization likely needed to produce the sound or to get the laryngeal sac to expand to transmit the sound ( $\mathrm{J}$. Reidenberg, pers. communication).

\section{Individual recognition by call characteristics}

Indirect observations suggest that female whales are responsible for the production of Scream calls (Kraus \& Hatch, 2001). CART and discriminant analysis were carried out on Scream calls to determine if there were detectable differences based on 1) age class of the female present in the group or 2) individual identity based on multiple recordings of the same female in different SAGs. Juveniles were defined as females that were 1-8 years of age and had never had a calf. Adults were females that were 9+ years or had already had a calf. A total of 300 calls were used for the juvenile vs. adult discrimination with 150 from SAGs with juvenile focals and 150 from SAGs with adult focals. Twenty calls from each of 15 different focal females were used for the individual identification analysis.

\section{Source Level}

\section{Bay of Fundy acoustic environment}

Figure 4.2 shows a map of the Bay of Fundy with depth contours. The region where all recordings were made ranged from 150-220 $\mathrm{m}$ in depth. Measurements reported by previous researchers indicate that the noise levels in the Bay of Fundy are 75-80 $\mathrm{dB}$ re $1 \mathrm{uPa}^{2} / \mathrm{Hz}$ at $500 \mathrm{~Hz}$ (Desharnais et al., 2000; Hay et al., Submitted). Sediment type 
varies, with LaHave clay, Scotian Shelf Drift, and sandstone bedrock making up the three predominant bottom types (Hay et al., Submitted). The area of recordings is predominated by varying depths of LaHave clay in the upper-sediment layer.

Temperature profiles indicate that the Bay of Fundy is expected to have a downward refracting sound speed profile in the summer months (Desharnais et al., 2000). These two physical properties will effect the transmission of these signals through the water and impact the long range propagation (Hay et al., Submitted).

\section{Recording sounds for source level estimates}

Sounds recorded in 2002 with accurate measurement of range to the animal producing the sound were analyzed to estimate the source level for different call types. Additional measurements were made of noise level to characterize the noise levels that may impact the communication of right whales in these groups. These noise level measurements were from recordings made at the same time as and in the same frequency band used to calculate received level of right whale sounds. Recordings were also made of whale watching and research vessels that approached SAGs to determine their source level. The hydrophone used for these recordings was an Hi-Tech HTI-94-SSQ hydrophone with a calibrated sensitivity level of $-170.4 \mathrm{~dB}$ re $: 1 \mathrm{~V} / \mu \mathrm{Pa}$ reported by the manufacturer based on comparison to a reference hydrophone. The recordings were made on a Sony DA-P1 DAT recorder. The same hydrophone and DAT were used for all source level measurements throughout the field season.

\section{Software calibration}

Source level measurements were made using the acoustic analysis program Canary (Version 1.5.1) (Charif et al., 1995). A $500 \mathrm{~Hz}$ sine-wave calibration signal was generated in Matlab 6.1 (Mathworks, 2002). The signal level output from the computer was measured with a calibrated oscilloscope. One 10 second calibration signal was generated with a measured .12 peak-to-peak voltage output from the computer. The RMS 
value, calculated by multiplying these values by $1 / 2 \sqrt{2}$, was $84 \mathrm{mV}$. This calibration sound were recorded into the same DA-P1 DAT recorder used for the right whale recordings to account for any signal level gain from the recorder. Given the sensitivity of the hydrophone, the calibration signal should correspond to the voltage produced when the hydrophone received a $148.9 \mathrm{~dB}$ re $1 \mu \mathrm{Pa}$ signal. The calibration sound was acquired into Canary from the DAT recorder (sampled at $44.1 \mathrm{kHz}, 16-\mathrm{bit}$ ) with a recording gain of 0.5 . The calibration for the file was set using the signal level calculated from the hydrophone sensitivity $(148.9 \mathrm{~dB}$ re $1 \mu \mathrm{Pa})$. This calibration was then used for all subsequent sounds acquired into Canary from the DAT recorder using the same acquisition settings (sampled at $44.1 \mathrm{kHz}, 16$-bit, gain 0.5 ).

\section{Acquisition of recordings}

Recordings from the Bay of Fundy were acquired into Canary using the same gain and calibration settings as the calibration signal. Received level was only calculated for sounds recorded from a whale at a known distance (by using array localization and a laser range finder). In general, tonal calls were measured for the average broadband signal level in $\mathrm{dB}$ re $1 \mu \mathrm{Pa}$ and in the frequency band of $2000-2200 \mathrm{~Hz}$. This second measurement at $2000-2200 \mathrm{~Hz}$ was selected to test whether right whales might be able to use the higher frequency harmonics of these calls for long distance detection when the low frequency components of the sounds would quickly be masked by low-frequency noise. Noise from vessels is generally at frequencies lower at $2000-2200 \mathrm{~Hz}$ and Scream calls commonly had strong harmonic energy in the $2000-2200 \mathrm{~Hz}$ band (Figure 3.2). Noise levels were also measured in the same frequency bands (broad band and in 2000$2200 \mathrm{~Hz}$ ) to allow for comparison to the signal levels. Noise levels were measured from selected recordings with no SAGs present, from recordings of vessels near SAGs and from a segment of recording $\sim 1$ second before each measured call. Source levels of broadband Gunshot sounds were measured using the entire frequency spectrum, and 
therefore cannot be compared directly with the source level measurements for the calls in the $2000-2200 \mathrm{~Hz}$ range.

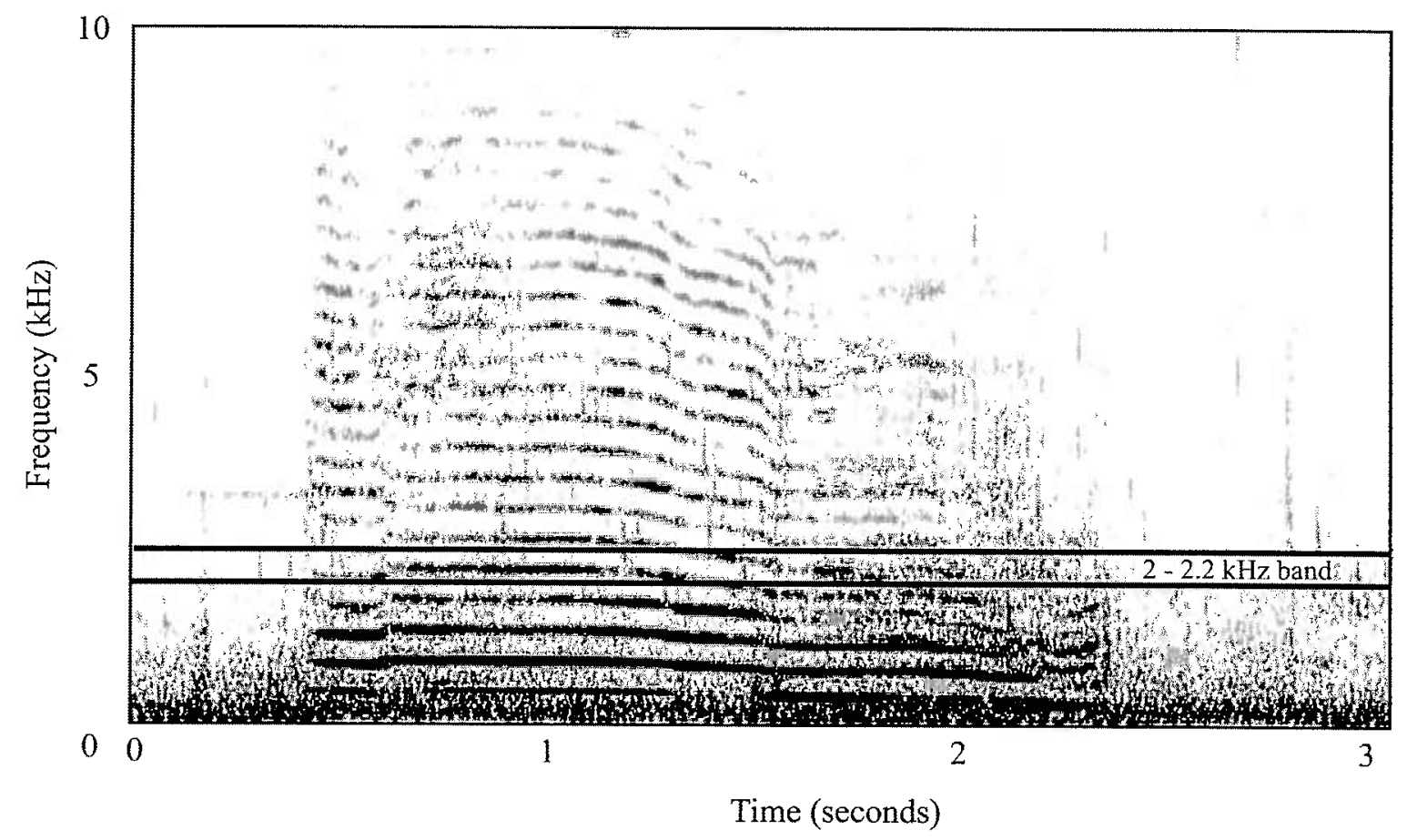

Figure 3.2. An example of a Scream call showing the $2-2.2 \mathrm{kHz}$ frequency band used for narrow band source level calculations. Note the signal energy in this frequency range and the increased levels of noise below the $2 \mathrm{kHz}$ section.

\section{Estimation of Source Level}

Source level (SL) and Transmission loss (TL) were estimated for calls by using the following formulae (Urick, 1983):

$$
\begin{gathered}
\mathrm{SL}=\mathrm{RL}+\mathrm{TL} \\
T L=20 \log (r)+\alpha * r * 10^{-3}
\end{gathered}
$$

Where $\mathrm{SL}=$ Source Level, $\mathrm{RL}=$ Received Level, $\mathrm{TL}=$ Transmission Loss, $\mathrm{r}=$ range in meters and $\alpha=$ the logarithmic absorption coefficient. The $\alpha$ portion of the calculation had negligible impact on the calculated TL at the ranges $(<200 \mathrm{~m})$ and frequency $(<3 \mathrm{kHz})$ in these measurements and was omitted from the final calculation of TL. The TL estimate $(20 \log (\mathrm{r}))$ represents spherical spreading loss and was selected 
because most recordings were made with the source closer to the hydrophone than the depth of the water. The average range for SL measurements was $150 \mathrm{~m}$ while the average water depth was $180 \mathrm{~m}$. The array was deployed at a depth of $5 \mathrm{~m}$. The whales producing the sounds were generally at a depth of approximately 2-5 m. A bellhop transmission loss model (M. Porter, Science Applications International Corp.) of the propagation loss for the Bay of Fundy showed agreement with the TL estimates to within $1 \mathrm{~dB}$ re $1 \mu \mathrm{Pa}$ (Personal communication, Adam Frankel).

\section{Estimation of range of detection}

A simple estimation for the range of detectability of Scream calls was made given the averages of the noise levels in both the broadband and $2000-2200 \mathrm{~Hz}$ band and the calculated SL for the Scream calls. The range was simply defined as the distance necessary for (Urick, 1983):

$$
\mathrm{TL}=\mathrm{SL}-(\mathrm{NL}-D I) \text {. }
$$

Where $\mathrm{TL}=$ transmission loss, $\mathrm{SL}=$ source level, $\mathrm{NL}=$ noise level and $\mathrm{DI}=$ directivity index. TL in this case is defined in terms of spherical spreading $(\mathrm{TL}=20 \log (\mathrm{r}))$ to the range of $200 \mathrm{~m}$ and cylindrical spreading $(T L=10 \log (\mathrm{r})$ ) beyond that range (Hay et al., Submitted). The DI is unknown for these right whale calls and was omitted from the equation, which is equivalent to setting $\mathrm{DI}=0$. This is a conservative estimate of range of detectability where $\mathrm{RL}=\mathrm{NL}$. Animals are often capable of detecting sounds that are received at levels similar to the overall noise levels (Richardson et al., 1995).

\subsection{RESULTS}

\subsubsection{Surface Active Group Recordings}

A total of 52 acoustic recordings were made between 1999 and 2002 (Table 3.2). Duration of acoustic recordings ranged from $2-132$ minutes. A total of 2992 sounds 
were selected for analysis from the recordings. The group size ranged from 2-15 whales. Six of the seven types of SAGs defined in Chapter 2 were recorded including malefemale pairs $(\mathrm{N}=3)$, one female with multiple males $(\mathrm{N}=11)$, multiple female groups $(\mathrm{N}=8)$, all-male groups $(\mathrm{N}=1)$, calf groups $(\mathrm{N}=9)$, pregnant groups $(\mathrm{N}=4)$ and unknown groups $(\mathrm{N}=16)$. There were no groups observed that were all female during the study.

\subsubsection{Sound classification}

\section{Call Types Defined}

Whale sounds recorded from SAGs were assigned to six classes based on differences in frequency, duration and/or individual responsible for sound production. These six classes correspond to those reported in Clark $(1982,1983)$. The classes defined in this thesis were, in order of occurrence, Screams, Gunshots, Noisy Blows, Upcalls, Warbles, and Down calls. The rationale for this classification is described in the methods. The number of each class and the characteristics of these sounds are described in Table 3.3. The corresponding call type described by Clark (1983) for Southern right whales are also included in the table. The center frequency and duration were measured in Acoustat and used the MSMED5 value for center frequency and the ENVUp5 value for duration. These two measurements most closely matched the observed center frequency and duration of the signal from visual inspection of spectrograms in Cool Edit Pro. 1.2 (Syntrillium Inc.). 


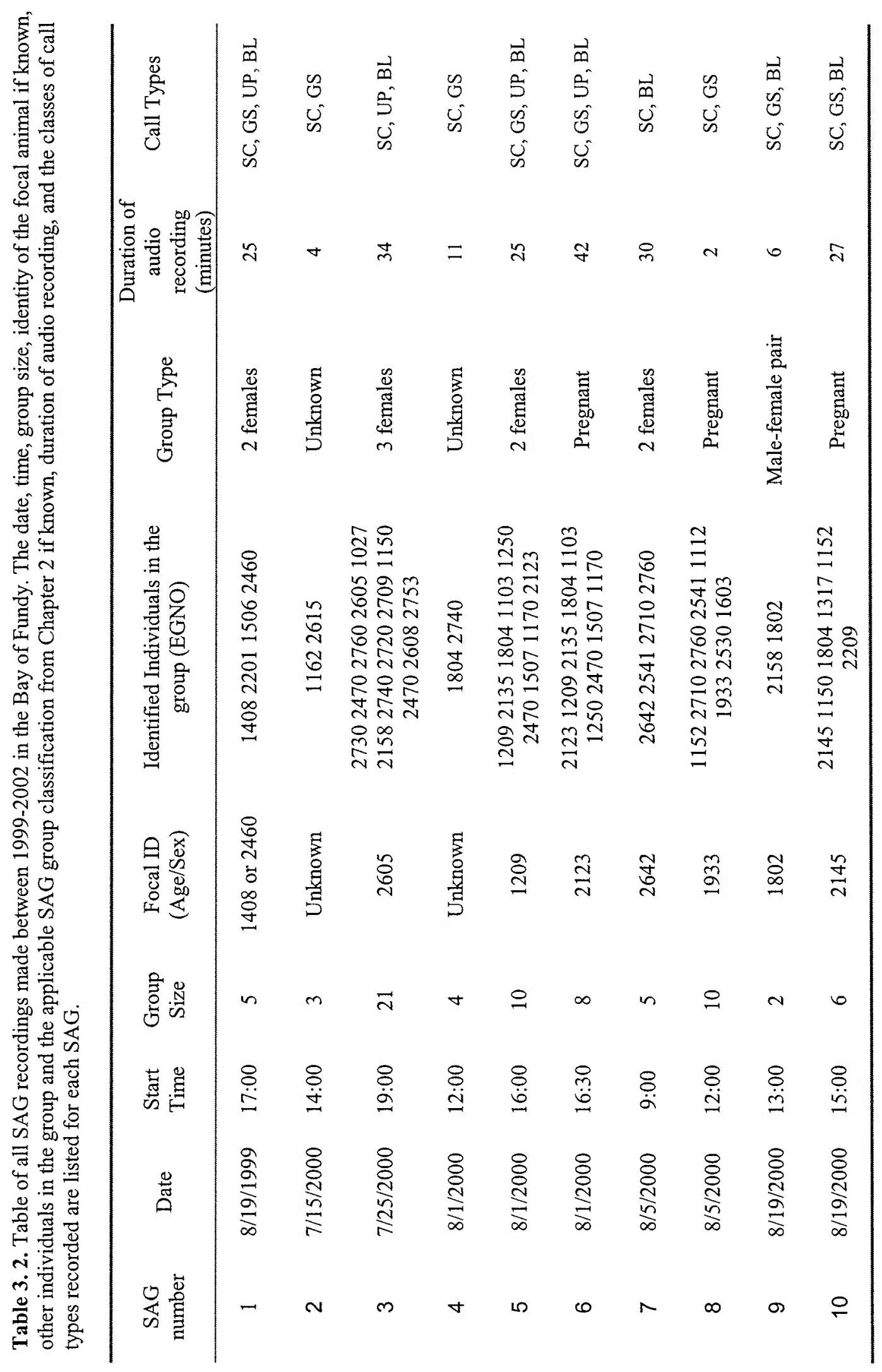




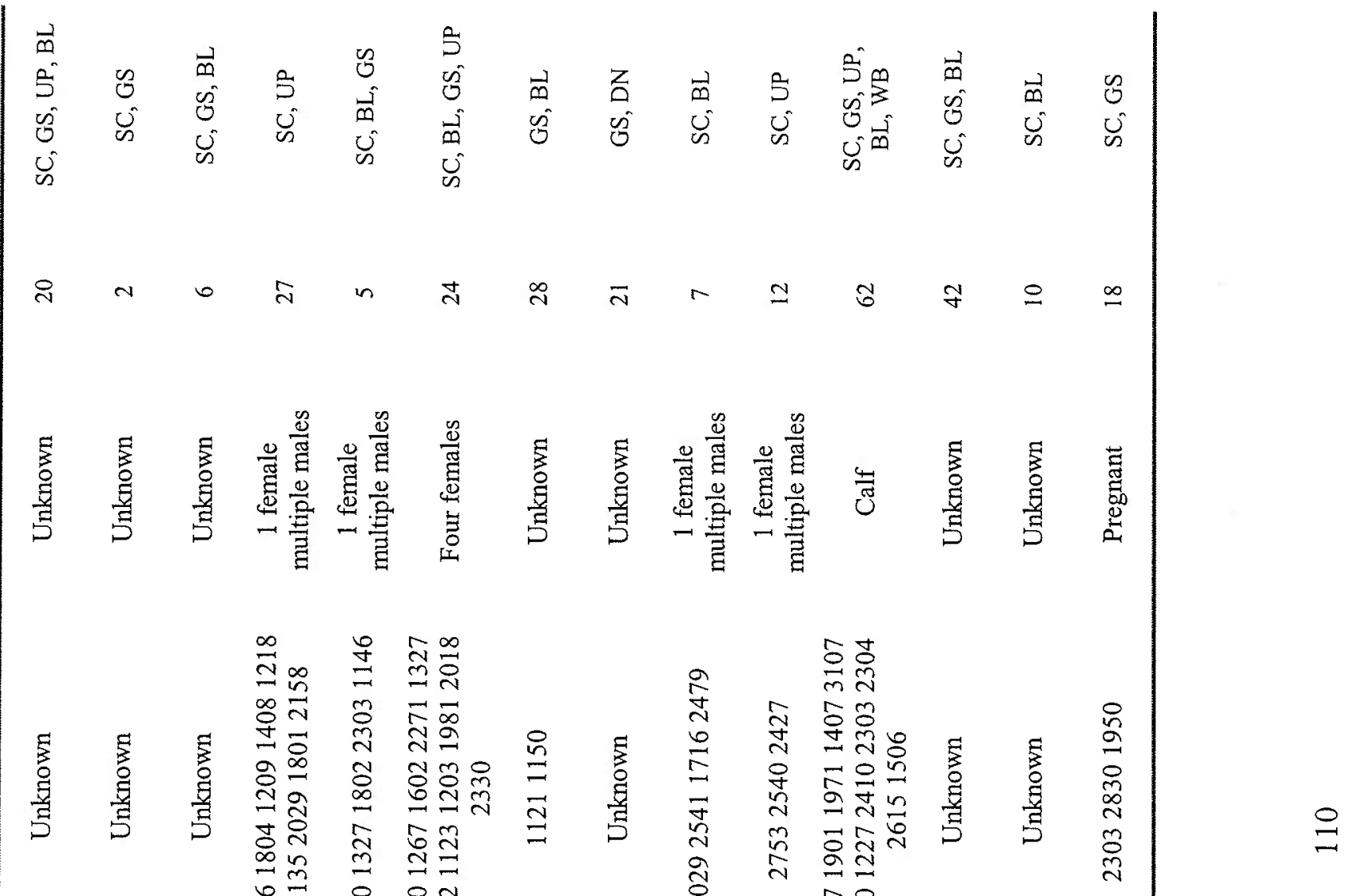

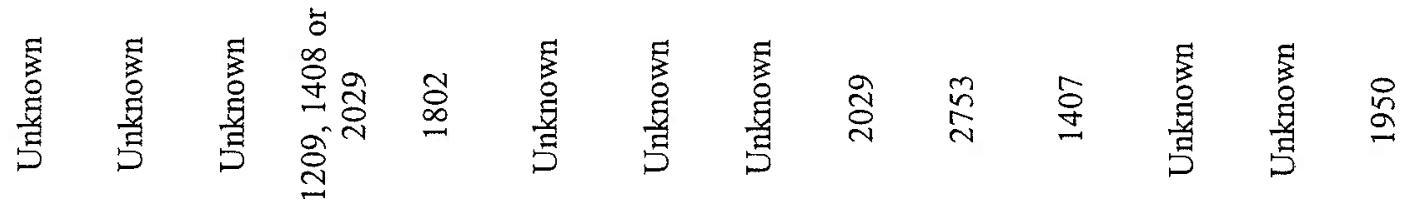

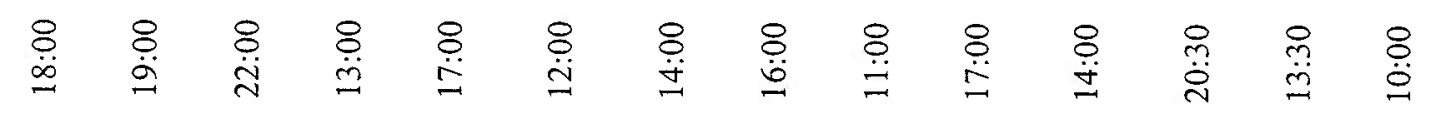

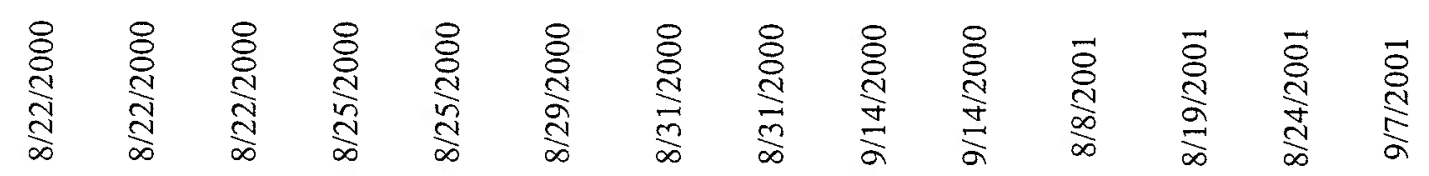

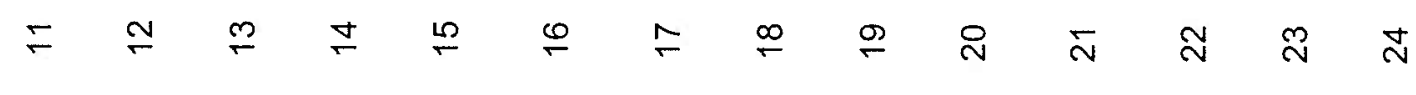




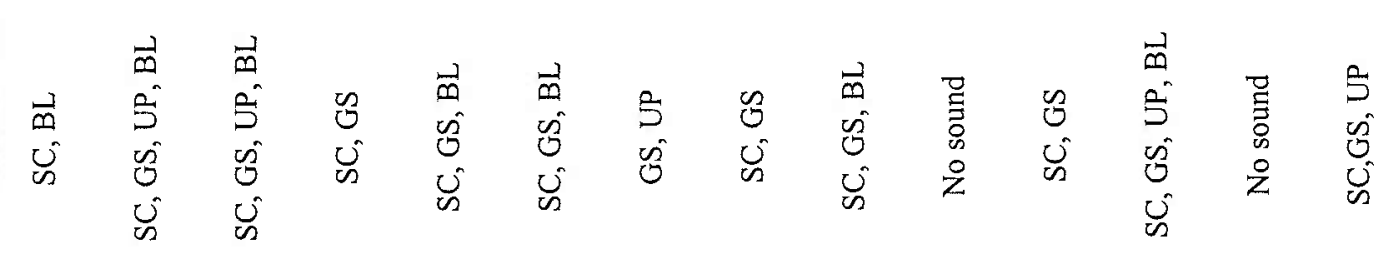

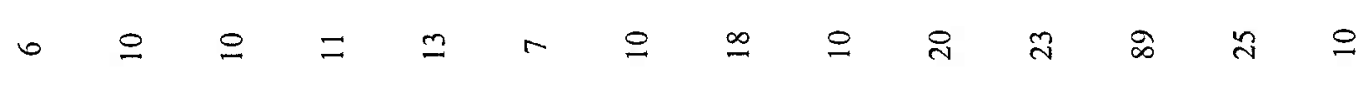

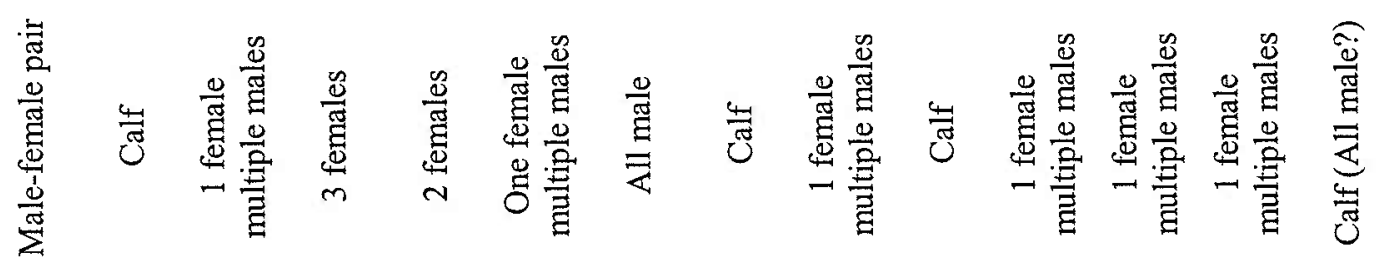

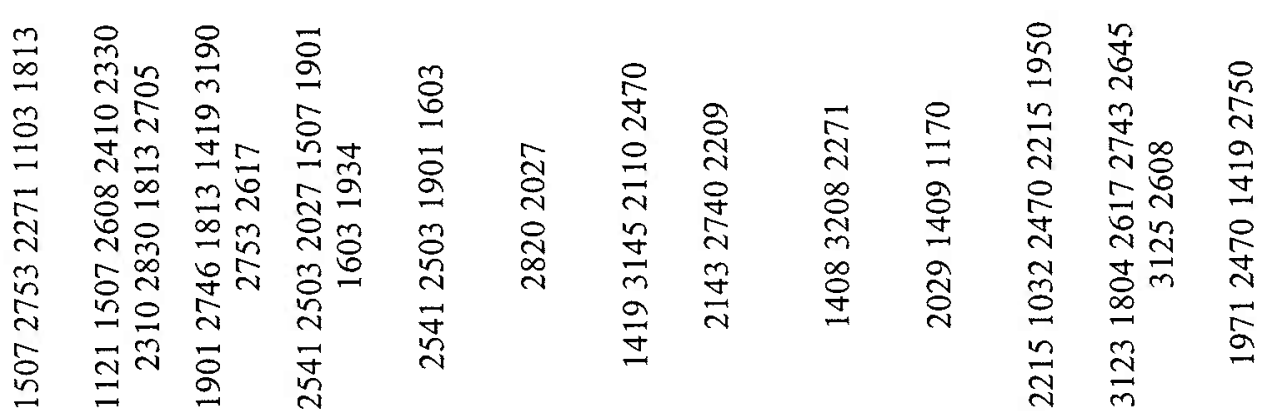

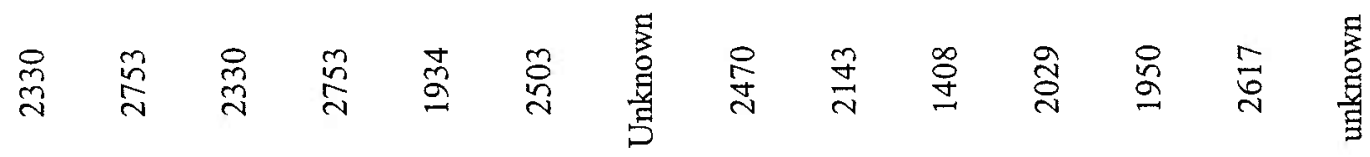

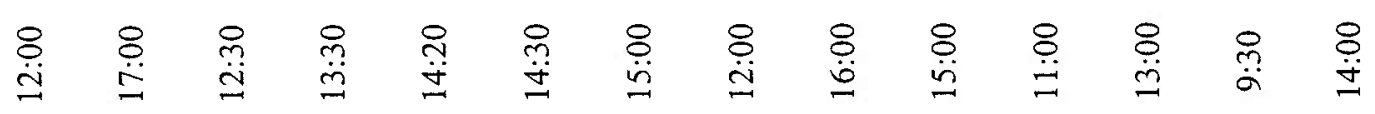

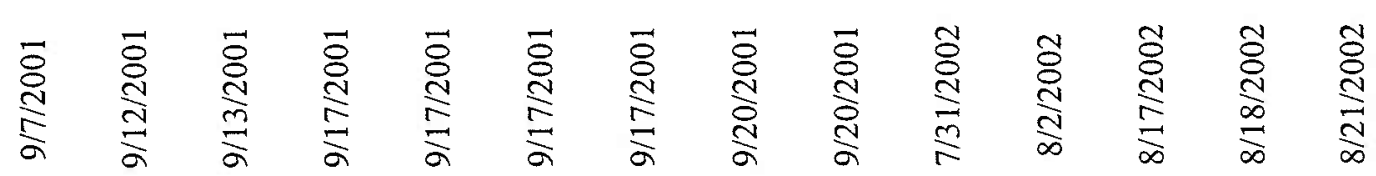
只 


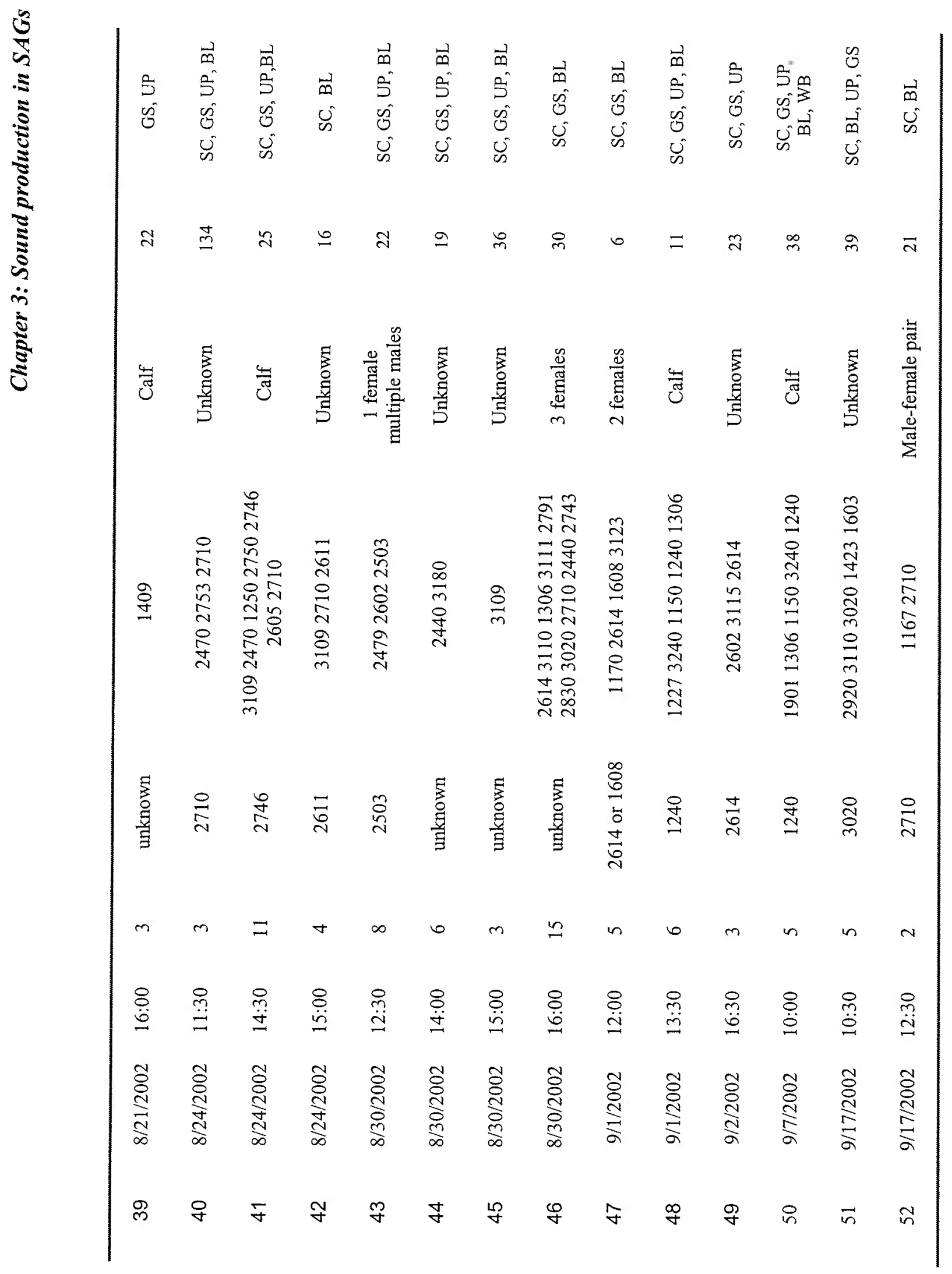


Table 3.3. Call types recorded in SAGs in the Bay of Fundy. Center frequency and duration were measured for 40 of each call type. The spectrograms are plotted on the same y-axis, with frequency from $0-4 \mathrm{kHz}$. The time values on the $\mathrm{x}$-axis vary ( 2 seconds for Scream and Warble, 1 second for other sound types). *- As defined by Clark (Clark, 1983; Clark, 1982).

\begin{tabular}{|c|c|c|c|c|c|}
\hline Sound class & $\begin{array}{c}\text { Total number in } \\
\text { sample }\end{array}$ & Description & & Spectrogram & $\begin{array}{l}\text { Southern right } \\
\text { whale types* }\end{array}$ \\
\hline Scream & 2208 & $\begin{array}{l}\text { Highly variable tonal calls } \\
\text { with harmonic structure. } \\
\text { May contain rapid } \\
\text { frequency modulation. Part } \\
\text { of the call may mix } \\
\text { broadband and tonal } \\
\text { signals. Major energy } \\
\text { ranges from } 200-2500 \mathrm{~Hz} \text {. } \\
\text { Duration ranges from } .3-5.1 \\
\text { seconds. }\end{array}$ & 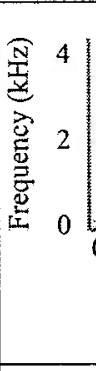 & $\frac{1}{0}$ & $\begin{array}{c}\text { High, Hybrid } \\
\text { and Pulsive calls }\end{array}$ \\
\hline Gunshot & 320 & $\begin{array}{l}\text { Noisy, broadband sharp } \\
\text { onset sound with major } \\
\text { energy from } 50-2000 \mathrm{~Hz} \text {. } \\
\text { Duration of the signal is .2- } \\
.3 \text { seconds, but the echo and } \\
\text { reverberation of the signal } \\
\text { can last from } 1-3 \text { seconds. }\end{array}$ & 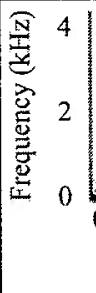 & 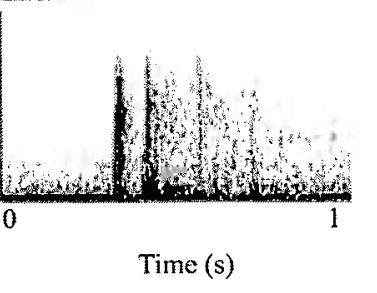 & $\begin{array}{l}\text { Underwater } \\
\text { slaps }\end{array}$ \\
\hline Noisy Blow & 245 & $\begin{array}{l}\text { Noisy, broadband signals } \\
\text { with major energy between } \\
100 \text { and } 1000 \mathrm{~Hz} \text {. Duration } \\
\text { ranging from } .8-2.1 \\
\text { seconds. }\end{array}$ & 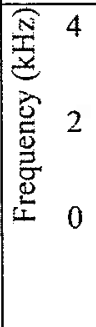 & $\frac{\left.\right|_{0}}{\text { Time (s) }}$ & Blows \\
\hline Upcall & 113 & $\begin{array}{l}\text { Low, tonal, FM sweeps } \\
\text { with major energy from } 50 \text { - } \\
200 \mathrm{~Hz} \text {. Duration ranges } \\
\text { from } .7-2.2 \text { seconds. }\end{array}$ & 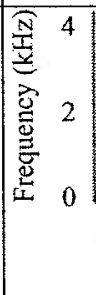 & $\frac{1}{\text { Time }(\mathrm{s})}$ & Up calls \\
\hline Warble & 61 & $\begin{array}{l}\text { High, tonal, frequency and } \\
\text { amplitude modulation } \\
\text { signals with major energy } \\
\text { from } 100-2000 \mathrm{~Hz} \text {. } \\
\text { Duration ranges from } .5-3.5 \\
\text { seconds. }\end{array}$ & 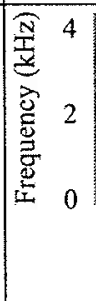 & 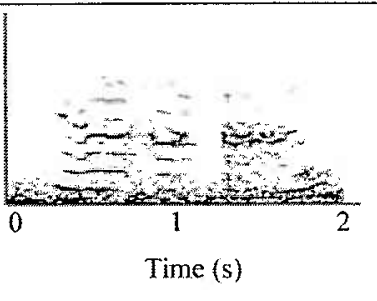 & Hybrid calls \\
\hline Downcall & 45 & $\begin{array}{l}\text { Low frequency tonal } \\
\text { upsweeps, with major } \\
\text { energy from } 100-400 \mathrm{~Hz} \text {. } \\
\text { Duration } .5-1.5 \text { seconds. }\end{array}$ & 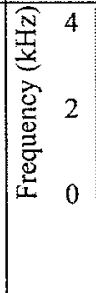 & $\frac{1}{0}$ & Down calls \\
\hline
\end{tabular}




\section{Cluster Analysis}

All 2992 whale sounds were processed using the Acoustat program (Fristrup \& Watkins 1994). Initially, 100 randomly selected samples representing 20 of each major sound type (Scream, Gunshot, Noisy Blow, Upcall and Warble) were Z-transformed to standardize the different Acoustat measurements. These samples were then subjected to cluster analysis, using a distance value of 10 as the cut off following the procedure used by Wright (2001) (Euclidean distance, Ward's minimum variance clustering). The resulting cluster tree is shown in Figure 3.3.

Types of sounds in each cluster

1. $20 \%$ Scream, $5 \%$ Upcall $25 \%$ Warble, $5 \%$ Blow

2. $20 \%$ Upcall, 10\% Scream $30 \%$ Warble

3. $15 \%$ Warble, $5 \%$ Blow

4. $20 \%$ Scream, $5 \%$ Upcall $5 \%$ Warble, $50 \%$ Blow

5. $40 \%$ Scream, $25 \%$ Warble, $10 \%$ Upcall

6. 50\% Gunshot, 5\% Blow, $10 \%$ Upcall

7. $40 \%$ Gunshot

8. 5\% Gunshot

9. $5 \%$ Gunshot, $35 \%$ Blow, $10 \%$ Scream, $50 \%$ Upcall

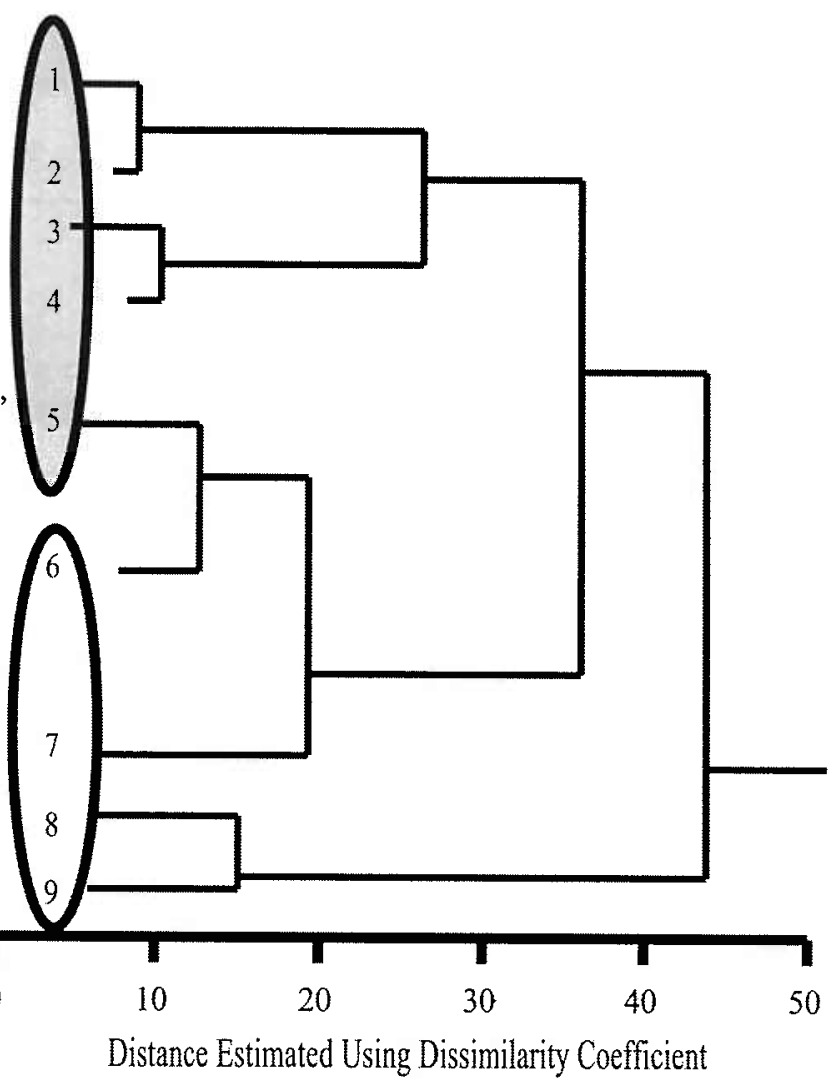

Figure 3.3. Results from cluster analysis of Z-transformed Acoustat measurements for a subset of 100 right whale sound types in Systat 10 using Ward's minimum variance clustering and Euclidean distance between clusters. The ovals represent the two major divisions between tonal calls and slap/blow sounds. The distribution of each sound type (as a percentage of the total number of each sound type in the processed sample) is listed on the left side of the tree. 
The final nodes and overall shape of the tree corresponds to the results reported by Wright (2001) with 9 terminal nodes of the tree. Note however, that several different classes of whale sounds can be found in particular nodes with a cut off value of 10 . Wright (2001) also found multiple types of sounds in several of the terminal nodes, although it is unclear what the exact distribution in the terminal nodes was. It appears that the major clusters found by Wright (2001) correspond well to those in Fig. 3.3 of this chapter both in terms of the dissimilarity coefficient and the distribution of sound types. A $5 \%$ classification value corresponds to a single sound being classified as a sample of 20 sounds of each type were used in the analysis. The cluster analysis found Noisy Blow sounds in clusters 4 and $6(50 \%$ in $4,35 \%$ in $9,5 \%$ in 1,3, and 6). The Gunshot sounds were found in clusters 6 and $7(50 \%$ in $6,40 \%$ in 7 and $5 \%$ in 8 and 9). The Scream sounds were found in clusters $1,2,4$, and 9 ( $40 \%$ in 5,20\% in 1 and 4, 10\% in 2 and 9). Warbles were found in clusters $1,2,3,4$ and 5 (30\% in 2, 25\% in 1 and 5, 15\% in 3,5\% in 4). Upcalls were found in clusters $2,3,4,5,6$ and 9 (20\% in 2, 5\% in 1,4,). Other tonal calls clustered in 1, 2 and $3(37 \%$ in 3,25\% in 1, and $9 \%$ in 2). Cluster 9 is puzzling as it combines both tonal and non-tonal sounds, with primarily Upcall and Noisy Blow sounds being grouped together. Overall, most of the tonal sounds were clustered in clusters 1-5, including more tonal Blow sounds, with the remaining Noisy Blows and Gunshots predominating clusters 6-9.

\section{Principal Components Analysis (PCA)}

The same randomly selection 100 calls used for cluster analysis were used for PCA. Twenty calls were selected from each major class of sounds used (Scream, Gunshot, Noisy Blow, Upcall and Warble). These 100 sounds were subjected to PCA using S-Plus 4 (Mathsoft, Inc.). The results of the PCA on the random sample of Ztransformed measurements reduced the original 108 Acoustat measurements to three principal component factors that explain $46 \%$ of the total sample variance. The results of this analysis were very similar to those reported in Clark (1983) with the first two principal components effectively separating Gunshot, Blow and Call classes (Figures 3.4 $\& 3.5)$. 


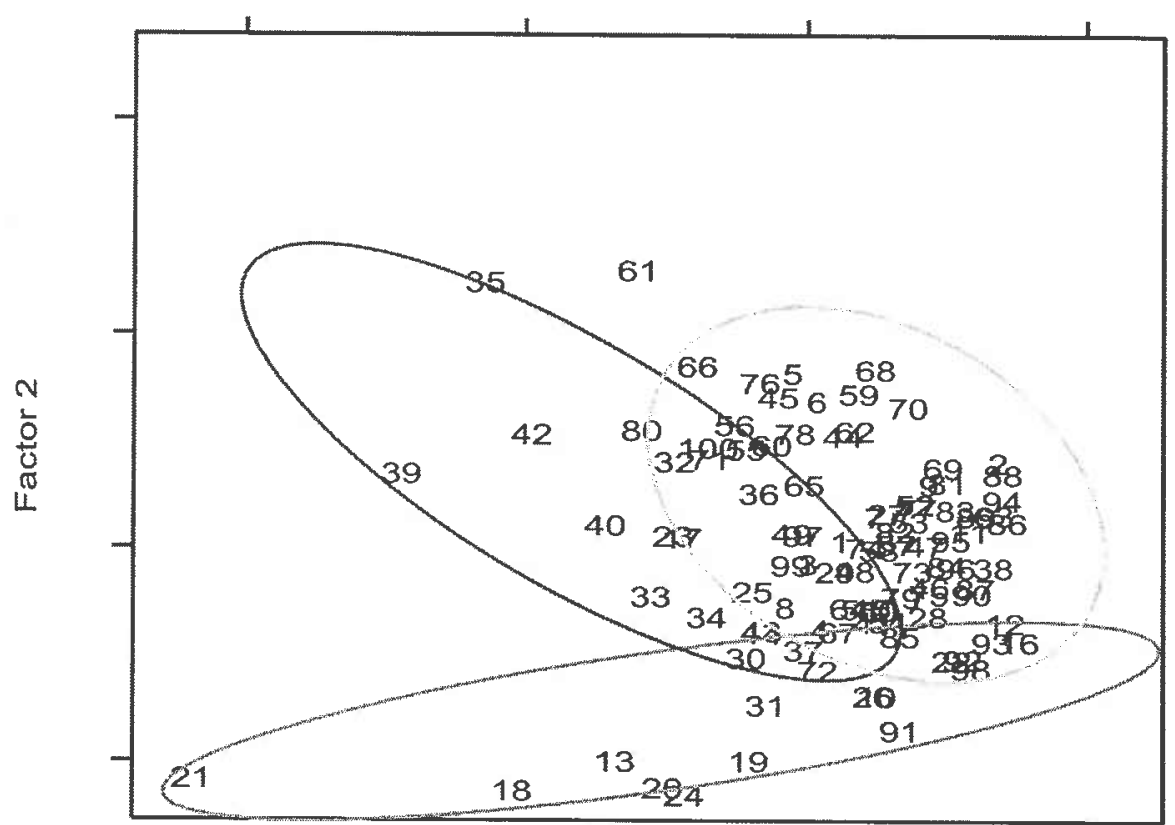

Figure 3.4. Results from PCA analysis showing the first two factors which preserve 38.6 percent of the variability. The bottom oval contains the Gunshot sounds, the middle oval contains the blow sounds, while the top oval describes the distribution of the tonal calls.

Factor 1

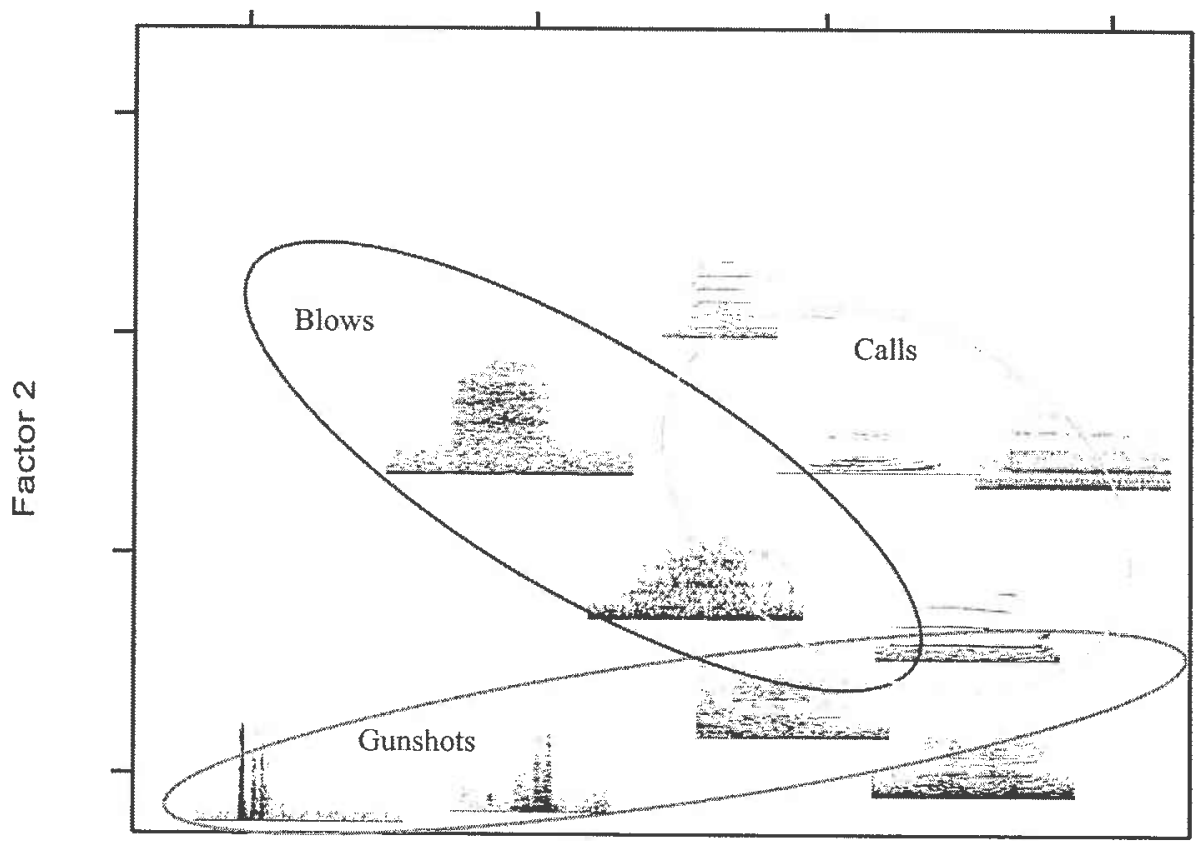

Figure 3.5. The same PCA results, showing the first two factors of the analysis with spectrograms for selected calls placed to help visualize the distribution of sounds. 
Classification of call types

Classification and Regression Tree (CART) analysis of all 2992 whale sounds did not confirm the proposed division of the whale sounds. This analysis resulted in division of the sounds into two main categories, Screams and Gunshots (Figure 3.6). CART analysis is sensitive to the number of examples of each type, and Screams and Gunshots dominate the number of whale sounds recorded from SAGs. Therefore a subset of the entire dataset was selected for a second CART analysis. 795 signals with the best signalto-noise ratio were selected for analysis. A random selection of 495 of these 795 signals was made, resulting the following dataset: 158 Screams, 127 Gunshots, 92 Noisy Blows, 87 Upcalls, and 31 Warbles. There were only 3 Downcalls with good signal to noise ratio, and these were not included in this analysis. This subsample resulted in better separation of call types as shown in Figure 3.7.

\section{CART of all 2992 sounds}

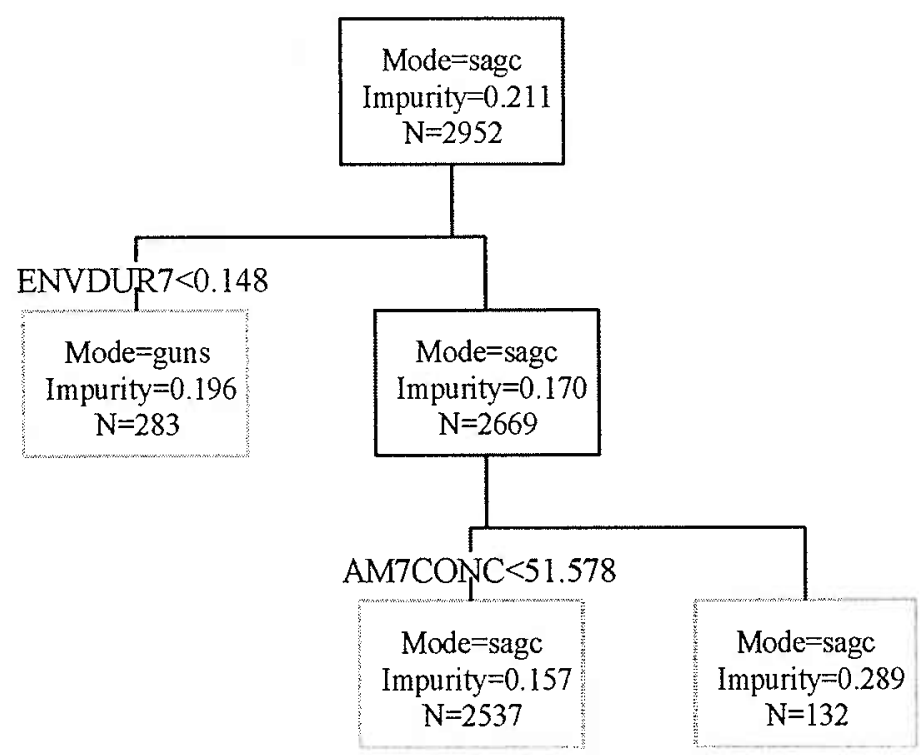

Figure 3.6. Classification Tree produced in Systat 10, using minimum split index value of 0.001 for all 2992 sounds. sagc $=$ Screams, guns $=$ Gunshot sounds. All other classes of whale sounds were included in the terminal nodes that were numerically dominated by Scream or Gunshot sounds. Each terminal node is shown in gray with the remaining "impurity" or misclassification remaining in the node. The Acoustat variable used for the branching decision is shown at each branch. 
CART of 495 sounds

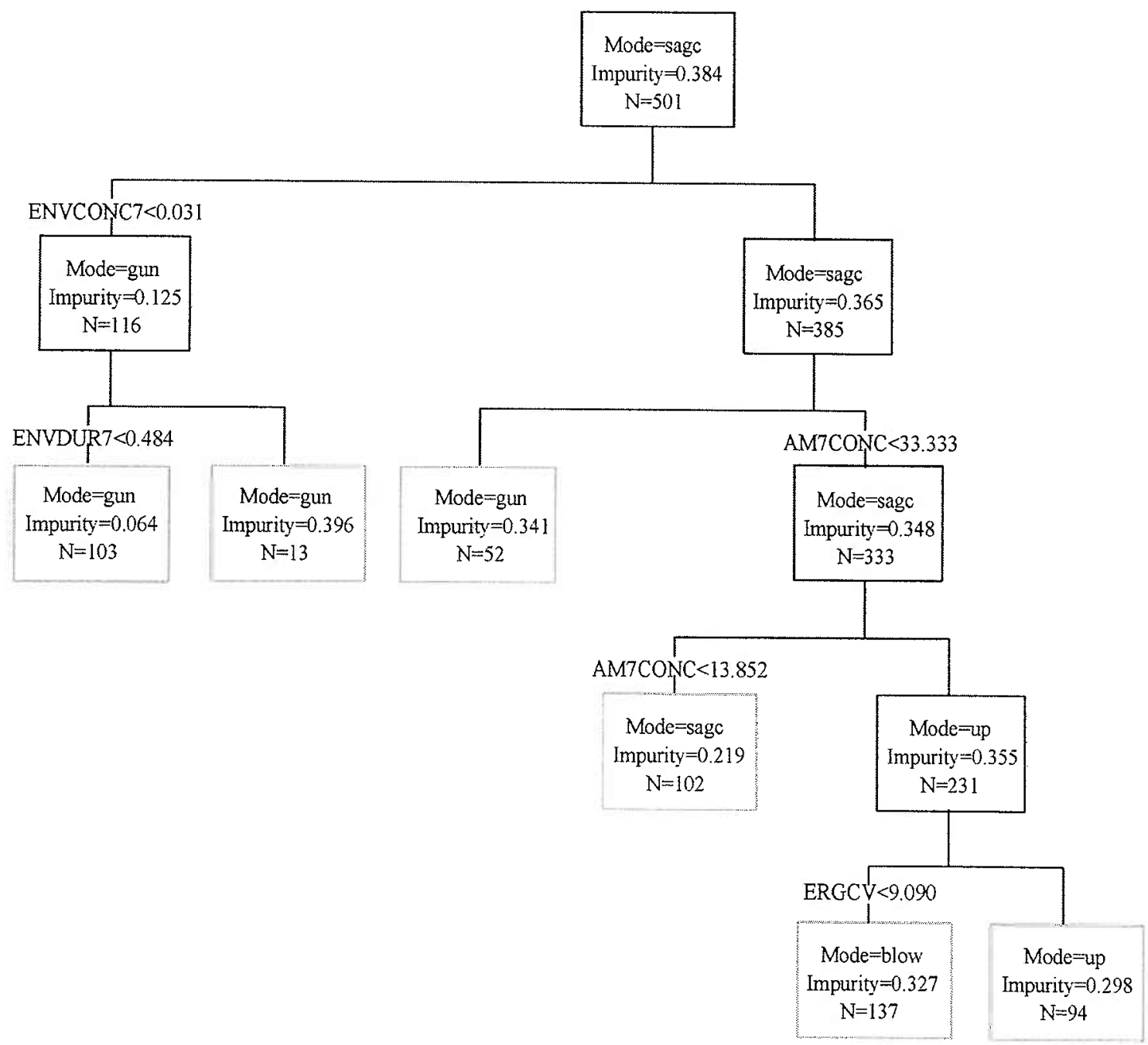

Figure 3.7. Classification tree of a subset of call types. The tree was constructed in Systat 10 with a minimum split index value of 0.010. sagc $=$ Screams, guns $=$ Gunshot sounds, up $=$ Upcall, blow $=$ Noisy Blow. Each terminal node is shown in gray with the remaining "impurity" or misclassification remaining in the node. The smaller sample size resulted in fewer misclassifications, but larger impurity values, as each misclassification had a more significant impact on the result. The Acoustat variable used for the branching decision is shown at each branch.

\section{Call type by group composition}

Each SAG in Table 3.2 lists which classes of whale sounds were documented during the recording period. Figure 3.8 shows the percent representation of each class of whale 
sound for different group compositions. The Scream calls dominate the call types in groups with single or multiple females and multiple males. They were recorded, but were rare, in the all-male groups. Scream production was almost equal to Gunshot sound production in the male-female pairs. Note that the Warble calls were only heard when calves were present, and primarily Upcalls and Gunshots were heard in the all-male group and one suspected all-male group with a calf (8/22/02). Upcalls were recorded in several different group compositions. These groups include the SAGs that would fit Clark's definition (1983) of sexually active such as the groups with a single female flanked by multiple males. The context in which Upcalls were commonly produced will be discussed in the individual sound production section.

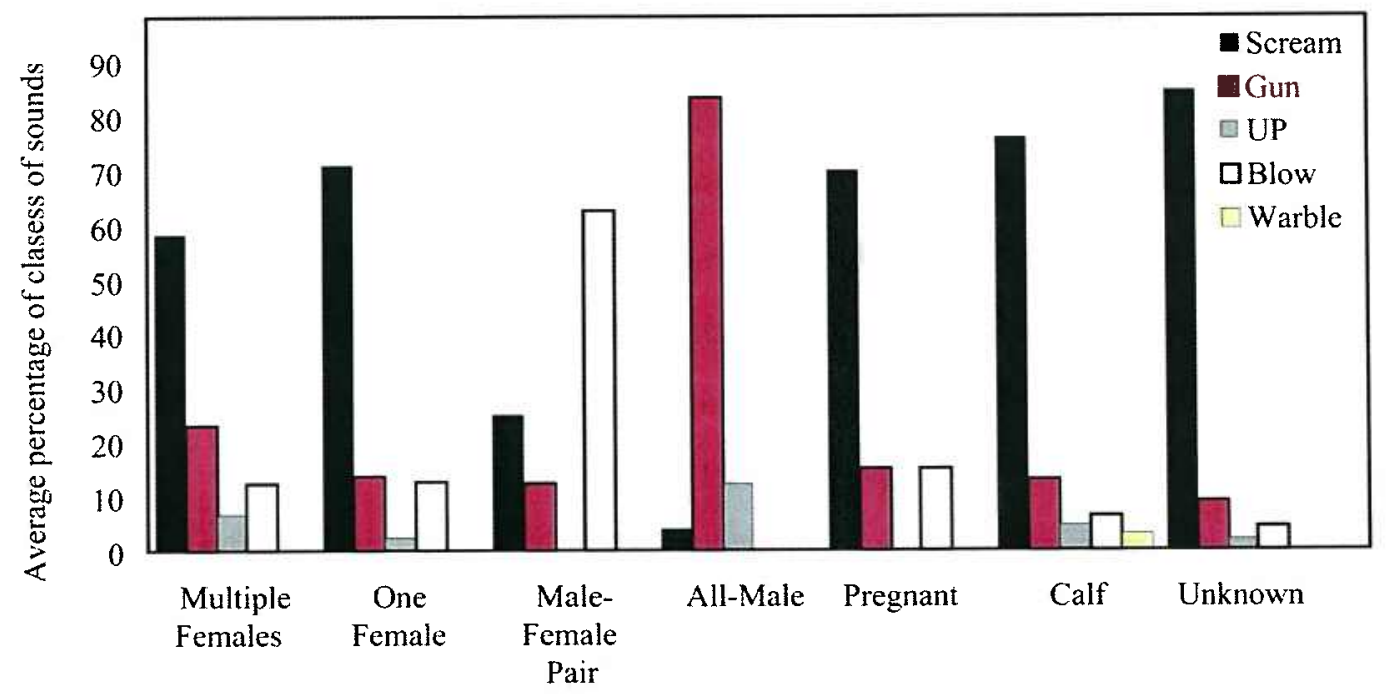

Figure 3.8. Average percentage of total calls produced of each class of calls by group composition.

\subsubsection{Call Timing}

Inter-call Intervals (ICI)

Timing of call production in SAGs in 42 of the $52 \mathrm{SAG}$ recordings was measured for the two main sound classes, Screams and Gunshots. The ten recordings omitted from this analysis either had recordings with no continuous recording longer than 1 minute in 
duration, background noise so high that making determination of call production was difficult, or no calls were recorded from the SAG during the observation period. A total of 2319 Scream calls and 408 Gunshot calls were inspected for this analysis, with the total number of Screams from each discrete SAG recording ranging from 0 - 381 (Average $=51, \mathrm{STD}=56$ ) and the number of Gunshot sounds from each SAG ranging from $0-63$ (Average $=13, S T D=16$ ). The time from the start of the recording to the first call, and the time from the last call to the end of the recording were not included in these analyses. Note that recordings with no Scream calls did have Gunshot sounds and that recordings with no Gunshot sounds did have Scream calls, allowing for these SAG recordings to be included in this analysis.

Scream calls and Gunshot sounds were characterized by periods of high rates of sound production indicated by short call intervals interrupted by periods of silence indicated by long call intervals (Figure 3.9). This resulted in a large proportion of very brief inter-call intervals (Figure 3.10a \& 3.10b). Inter-call intervals for Screams ranged from 0 - 4592 seconds. Screams were only absent from one recording (SAG 39). Gunshots were also variable in production with intervals between Gunshots ranging from 0-1592 seconds. Gunshots were not recorded in ten of these SAGs, with only 32 of the 42 analyzed SAGs having more than one Gunshot recorded.

The log survivorship analysis for Screams and Gunshots resulted in a bout criterion interval $(\mathrm{BCI})$ for Screams $=90$ seconds (Figure 3.11a) and a BCI for Gunshots $=120$ seconds (Figure 3.11b). The values for inter-call and inter-Gunshot intervals within bouts, and between bouts, are summarized in Table 3.4. The within bout intersound intervals are significantly different between the Scream and the Gunshot sounds (ttest; $\mathrm{t}=-11.695, \mathrm{P}=<0.00001$ ) with the mean inter-call interval being significantly lower for Screams than for Gunshots. However, the between bout intervals are not statistically different for the two sound types ( $\mathrm{t}$-test, $\mathrm{t}=-0.662, \mathrm{P}=0.510$ ). Figure 3.12 shows how the BCI divides the inter-call intervals for one series of calls from SAG 40 . 
SAG $40(\mathrm{~N}=380)$

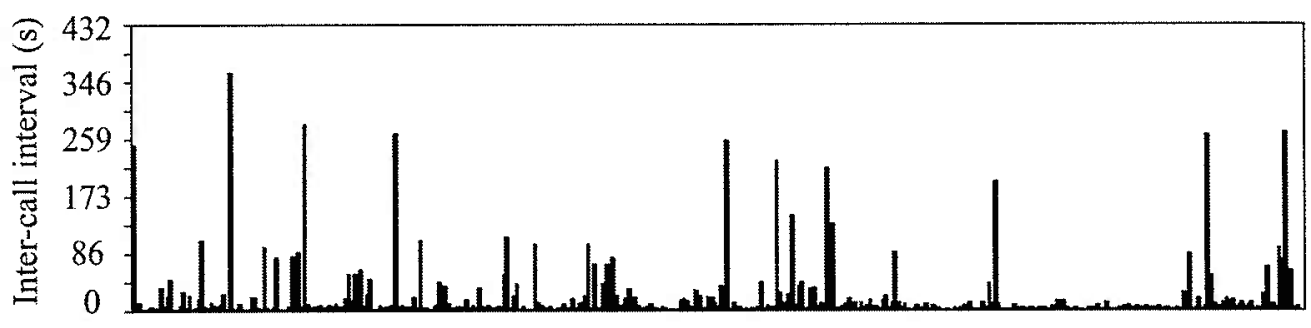

Successive Screams

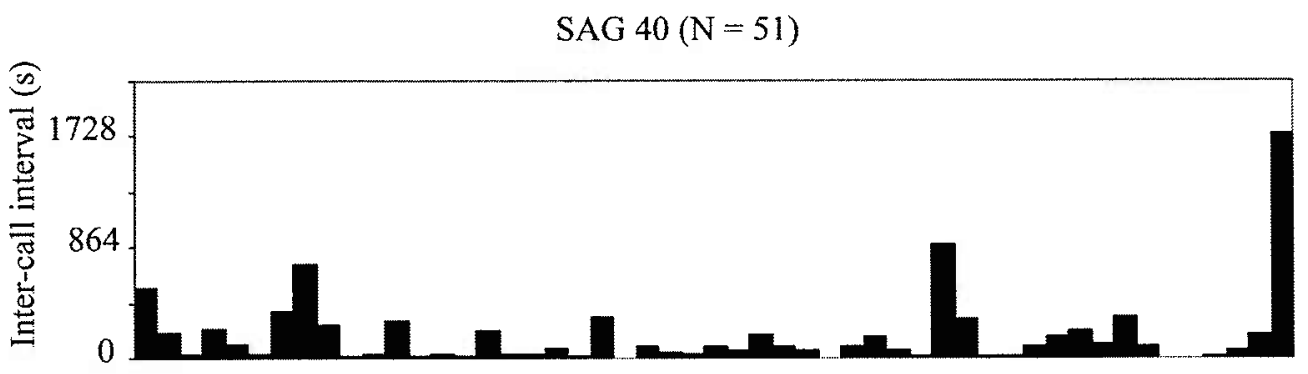

Successive Gunshots

Figure 3.9. Figure illustrating distribution of successive inter-call intervals between Screams and Gunshot from SAG 40 on Table 3.2. $\mathrm{N}=$ number of Screams. The plot indicates the successive intervals between Screams and Gunshots in the recordings from this SAG. The plots indicate periods of short inter-call intervals with longer interval lengths occurring periodically.

a)

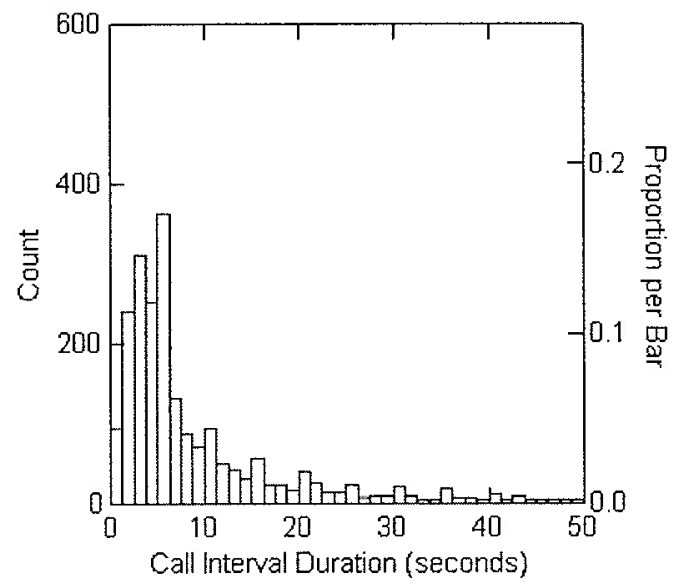

b)

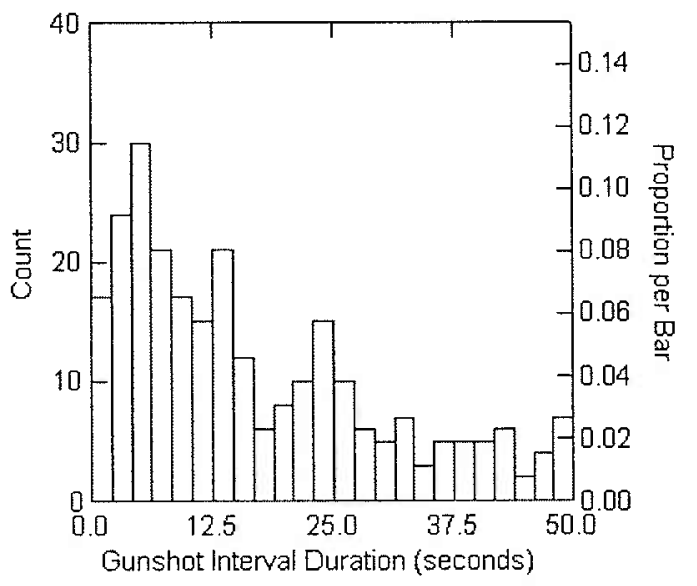

Figure 3.10. Plots of counts of (a) inter-call intervals for Screams and for (b) inter-gunshot intervals. Most of the intervals for both types were under 50 seconds in duration. 
a)

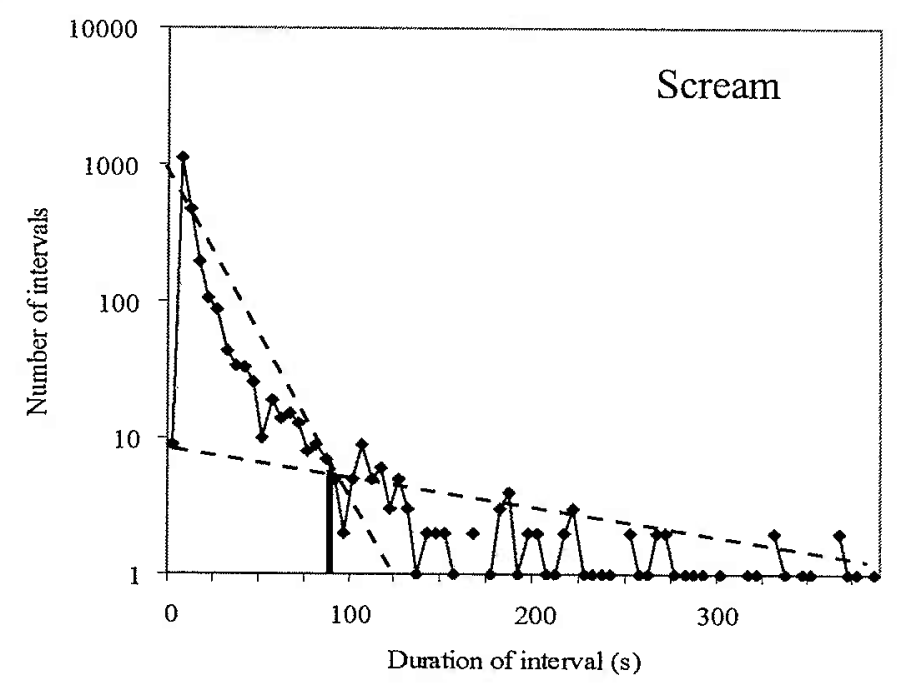

b)

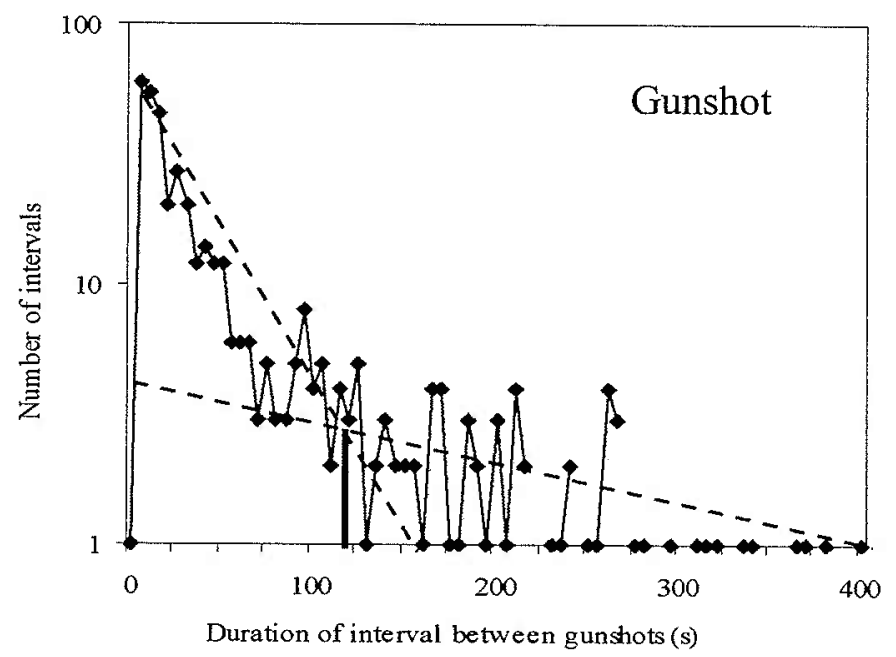

Figure 3.11. a) Log survivorship plot of inter-call interval between Screams. Dashed lines indicate the approximate slope for two parts of the curve. The black solid line indicates the bout criterion interval value determined from the intersection of the two slope lines. b) Log survivorship plot of inter-Gunshot interval between Gunshots also showing the bout criterian interval value. 
Table 3.4. Descriptive statistics for inter-call and inter-Gunshot intervals both within bouts defined by the measured bout criterion interval and between bouts of sound production.

\begin{tabular}{c|cccc}
\hline & $\begin{array}{c}\text { Screams within } \\
\text { bout }\end{array}$ & $\begin{array}{c}\text { Screams between } \\
\text { bouts }\end{array}$ & $\begin{array}{c}\text { Gunshots with } \\
\text { bouts }\end{array}$ & $\begin{array}{c}\text { Gunshots between } \\
\text { bouts }\end{array}$ \\
\hline $\mathrm{N}$ & 2214 & 107 & 340 & 90 \\
Minimum (s) & 0 & 94 & 0 & 121 \\
Maximum (s) & 90 & 4952 & 118 & 1986 \\
Mean (s) & 11.2 & 283.6 & 29.3 & 354.8 \\
Standard Dev. (s) & 14.7 & 507.8 & 29.5 & 332.0 \\
\hline
\end{tabular}

SAG $40(\mathrm{~N}=380)$

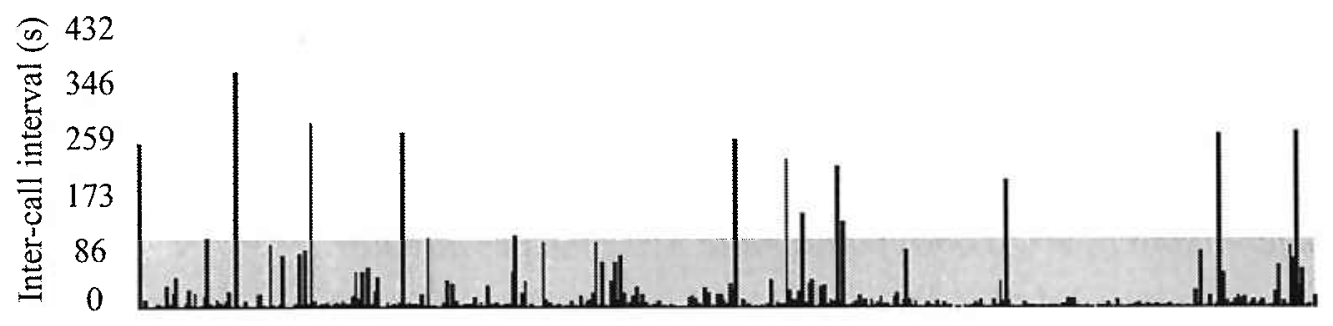

Successive screams

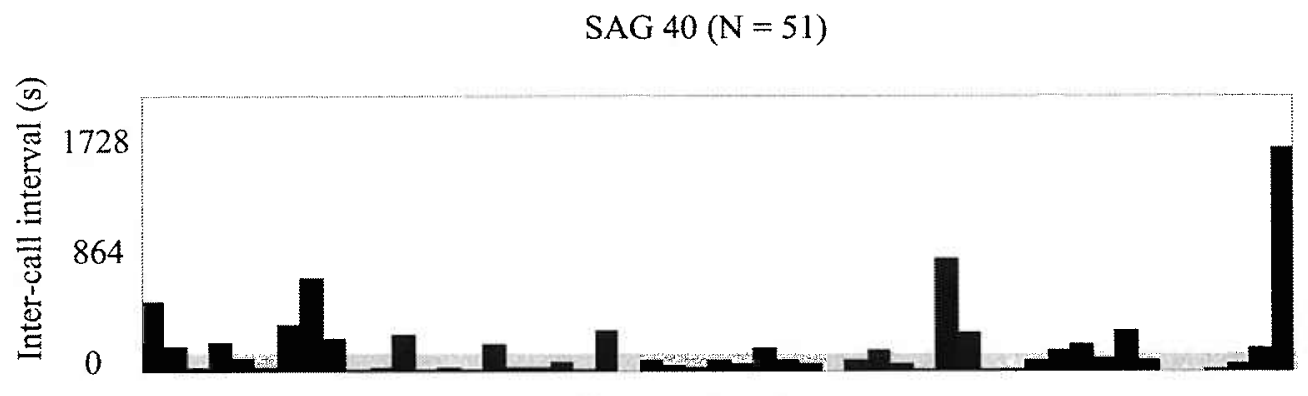

Successive gunshots

Figure 3.12. The graphs from Fig. 3.9 illustrating distribution of successive inter-call intervals between Screams and Gunshot from SAG 40 on Table 3.2. $\mathrm{N}=$ number of each sound type. The gray box encloses the with-in bout interval based on the BCI for the Screams and Gunshot sounds. There were 12 bouts of Screams and 10 bouts of Gunshots in the recordings from this SAG. Each discrete bout is separated by a call length interval that is higher than the $\mathrm{BCI}$. 
Call rate per minute (CPM)

Call rate was also calculated as the number of Screams or the number of Gunshots produced within one minute of a recording session. This analysis was carried out to determine maximum sustained rates of sound production, which can be missed in the inter-call interval (ICI) analysis. While the inter-call interval results in fewer long intervals, the calls per minute results in fewer high rates of call production. This shift in emphasis, from the duration between sounds (ICI) to the clustering of sound production $(\mathrm{CPM})$, may be more important than the absolute timing of each sound produced to discriminate between group compositions. 927 minutes of SAG recordings were analyzed. The number of minutes of recording analyzed from each SAG ranged from 2130 minutes (Ave. $=18 \mathrm{~min}$.). The average number of Screams was 2.6 (range 0-20, SD $=3.481$ ) Scream calls/minute (Figure 3.13a). The average number of Gunshots was 478 (range 0-22, $\mathrm{SD}=1.2$ ) Gunshots/minute (Figure 3.13b). This result is consistent with the inter-call interval finding of higher Scream rates than Gunshot rates overall in SAGs. The difference in rates between the two types of sounds was highly significant (MannWhitney Test using a random selection of 100 call rate values for each call type, Ustatistic $=6845.5, \mathrm{P}<0.0001)$ with more Screams per minute.
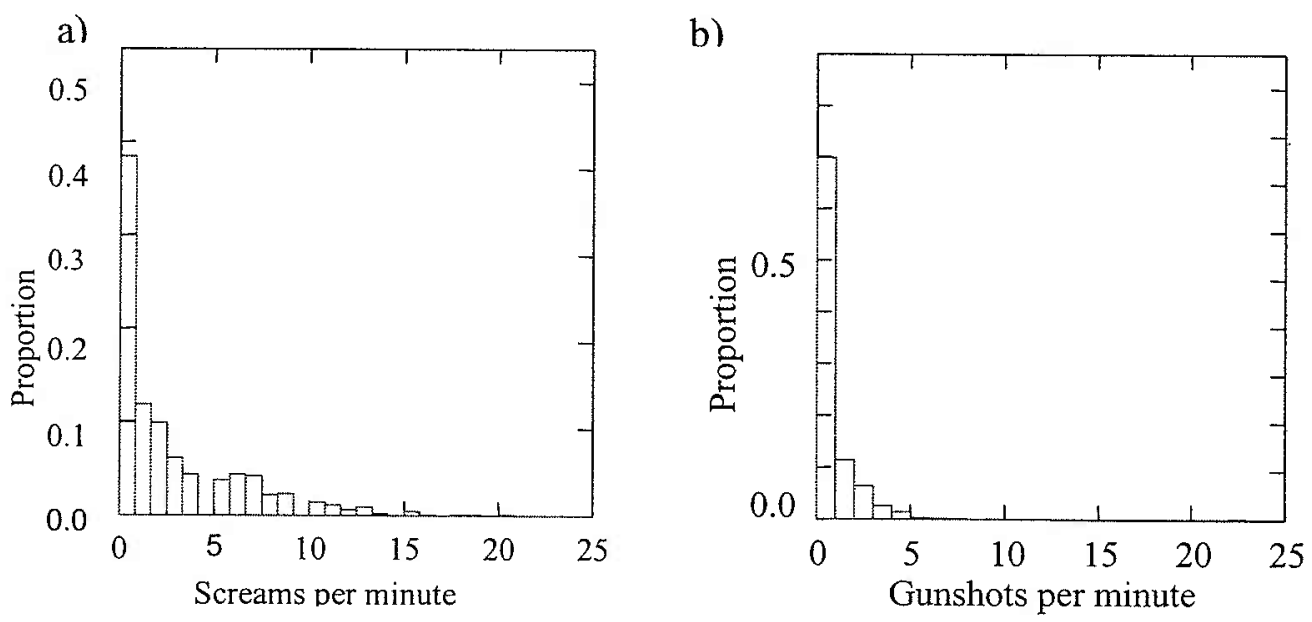

Figure 3.13. Proportion of rates of calls per minute for a) Screams and b) Gunshots. 
Call timing related to group size

Calculations of the average ICI within bouts, as well as the minimum and average between-bout intervals using $\mathrm{BCI}$ to define the minimum between bout intervals, were compared to group size for each SAG. The correlations were not statistically significant for any of these parameters ( $r=-.076$ for average inter-scream interval vs. group size). The only correlation was found between maximum Gunshot CPM and group size, with a slight positive correlation $(r=0.508$ ) (Figure 3.14). The Scream call per minute correlation value was $r=0.208$.

a)

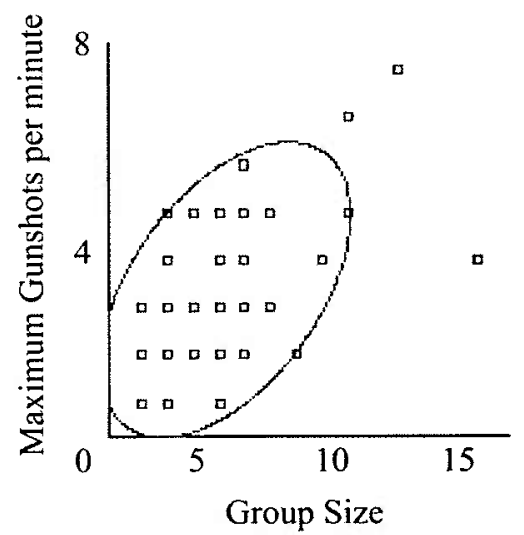

b)

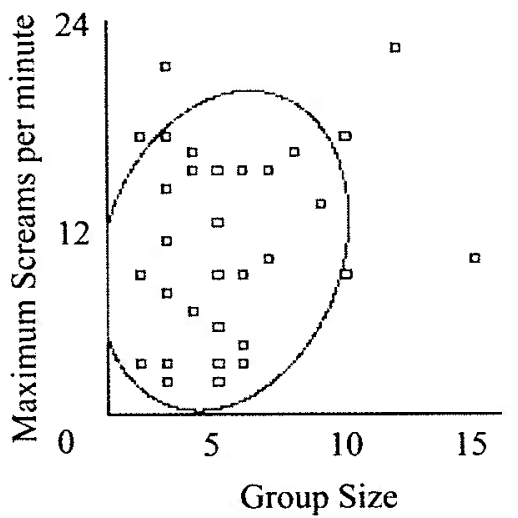

Figure 3.14. Correlation of group size versus a) the maximum Gunshot sound per minute rate. The correlation value was $r=.508$. b) Maximum Scream call per minute rate for each $\mathrm{SAG} \mathrm{r}=.208$.

\subsubsection{Individual sound production}

\section{Array Data}

Array recordings were made of 17 of 19 of the SAGs recorded in 2002. The 2 SAGs from 2002 with no array recordings occurred on 9/1/02 when sea state conditions were too high to deploy the array. The duration, signal-to-noise ratio, and number of calls recorded in each session varied. Simultaneous failure of the behavioral observer's microphone and Datascope from the middle of the day of 8/24/02 through 8/30/02 prevented range and bearing observations for the array analysis on those days (SAGs 41- 
46). Several attempted array recordings resulted in no sounds being recorded as the SAG ended by the time the array was deployed after photo ID (e.g. SAGs 34, 37, 49 and 52). These problems resulted in useable recordings being limited to seven SAGs.

Calibration recordings of vessels yielded a range of bearings to the source and these data are shown as the difference between each array bearings and the corresponding visual bearing to the source (Figure 3.15). The variation between visual bearing to the vessel and the array bearing to the vessel sound source ranged from 3-17 degrees. As predicted by theory, the array bearings were less accurate near either end of the array compared to perpendicular to the array (Figure 3.1).

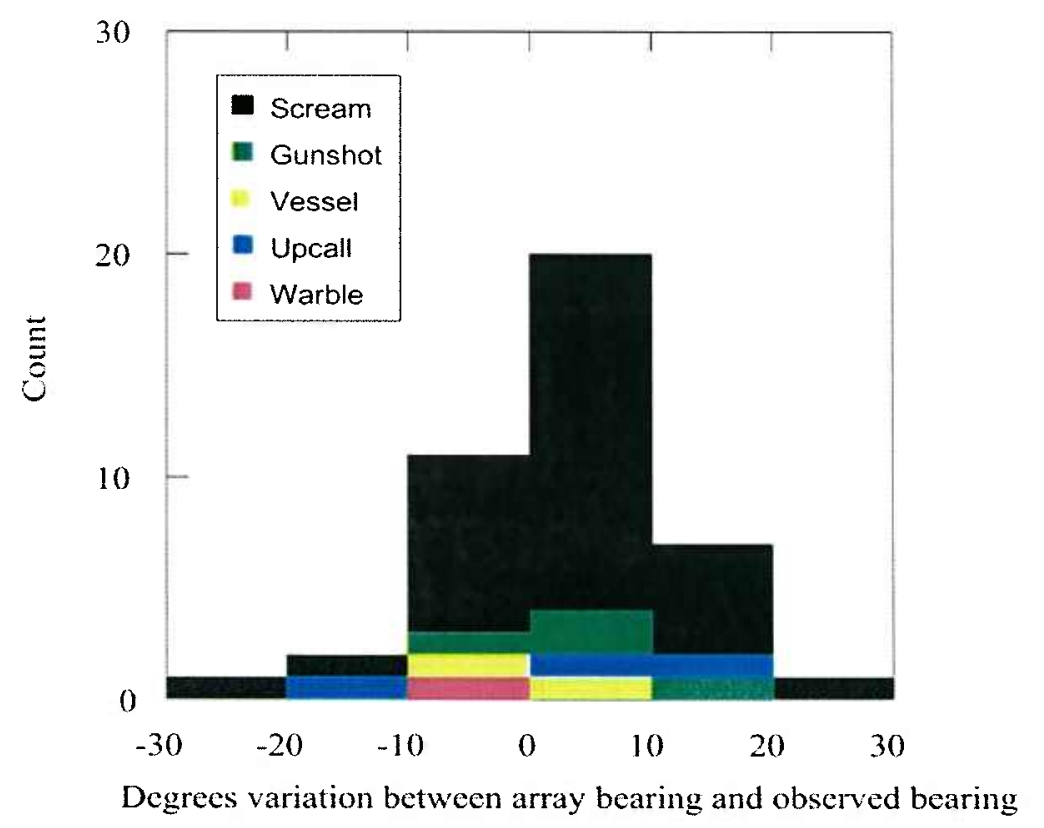

Figure 3.15. Distribution of differences in bearing to sounds from the array and observed bearings to sources.

Separations of the focal female from the SAG were never observed while the array was in the water and recording (total number of separations observed at other times $=5$ ). Therefore it was not possible to assign production of any call type definitively to the focal female. However, bearings to Scream calls were always consistent with the position of the focal female, never occurring when the female was not present in the SAG and never originating from a bearing other than the SAG (Figure 3,16). Gunshot sounds were 
never documented as being produced from a bearing other than that of the SAG during these groups. Therefore, males and/or females may be producing these sounds in the groups (Figure 3.16). Bearings to Noisy Blows were easily linked to visible exhalation of a whale and were produced both by the focal female and other whales present in the group.

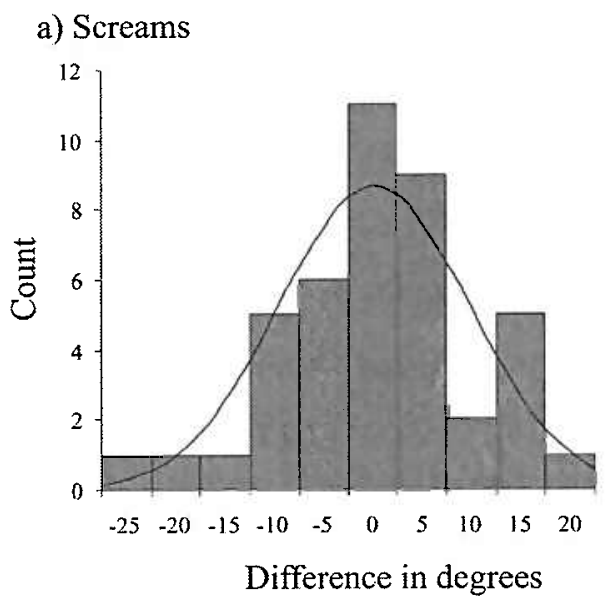

b) Gunshots

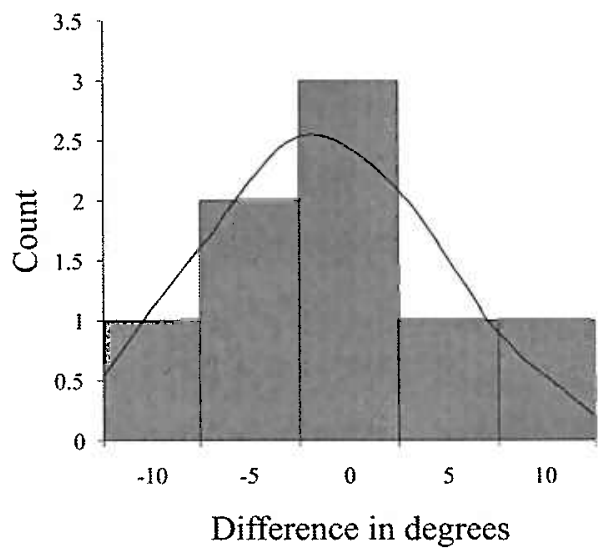

Figure 3.16. Difference in degrees between the array bearing to the sound source of a) Screams and b) Gunshots and the visual bearing to the center of the SAG being recorded.

Upcall production coincided on five occasions with the separation of adult males from the $\mathrm{SAG}$, either when the male was joining the $\mathrm{SAG}$ or when the focal female was on a dive. Good bearings to adult males were obtained on all five occasions (Figure 3.17). The males produced these calls during what appeared to be searching behaviors or when approaching a SAG for the first time. On two occasions when calves were present in the group, males were seen to stay close to the calf while the female was on a dive. Upcalls were recording in both these incidents and it is unclear whether the calf or the male produced them because the two whales were too close together.

Two bearings were obtained to Warbles on one occasion when a calf was alone at the surface while the rest of the SAG was down on a dive (Figure 3.17). Warble sounds have only been heard in SAGs with calves present, and these bearing data confirm that calves do produce these sounds. The aural quality of these Warbles are generally higher in pitch than most Scream calls, often longer in duration and broken up with many pauses 
in the sound production of what seems to be a single call. The call rate for these Warbles is approximately the same as that seen for Scream calls.

a) Upcalls

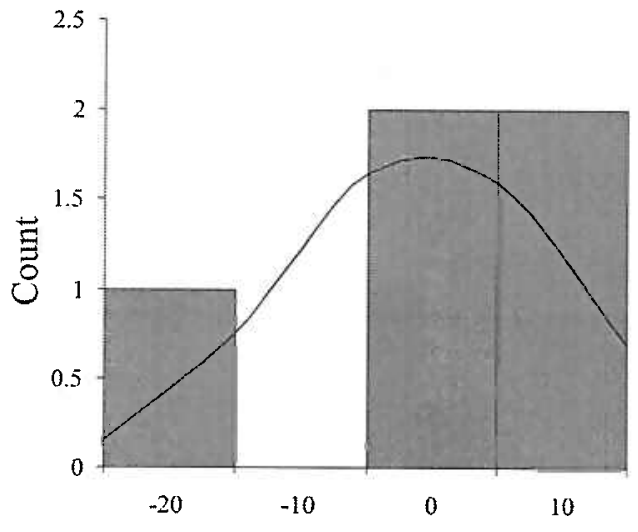

Difference in degrees b) Warbles

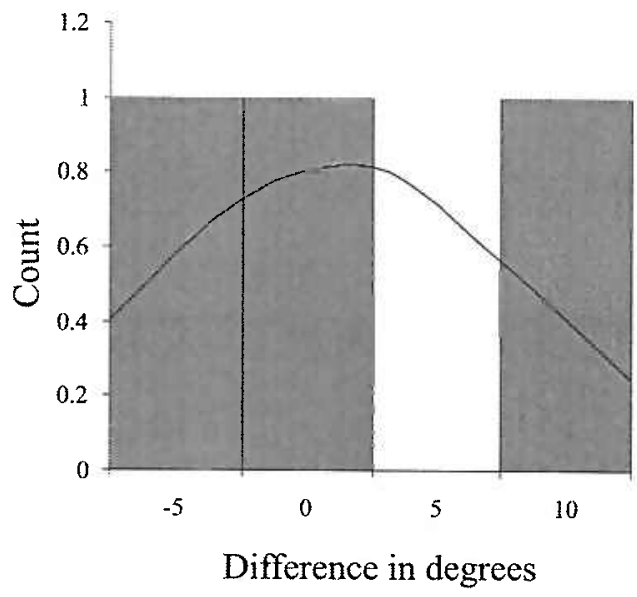

Figure 3.17. Difference in degrees between the array bearing to the sound source of a) Upcalls and b)Warbles and the visual bearing to individual whales outside of the SAG. In the case of Upcalls, all bearings are to lone adult males either at the surface while the SAG is down or joining the SAG as an approacher. The warbles were all documented as bearings to a lone calf at the surface when the rest of the SAG was down.

\section{Video Data}

Video recordings were used to provide additional data addressing the issue of which individual produces particular sounds in the SAGs. Counts were made of the number of breaths taken by the focal female and the total number of Scream and Gunshot sounds produced during the recording. Additionally the number of Screams and Gunshots produced simultaneously with the focal female breathing were counted for each SAG with video recordings made with a hydrophone recording directly into the video camera (Table 3.5). These results indicate the female was not breathing while Scream sounds were produced. Kraus \& Hatch (2001) reported similar results, never observing focal females breathing when sounds were recorded. The observation that no Scream calls were produced simultaneously with the focal female whale breathing is consistent with the hypothesis that the female produces these sounds. 31 Gunshot sounds being produced simultaneously with the focal female whale breathing indicate that the whales other than the focal female produce these sounds. Note that these results do not eliminate the 
possibility that males may also produce Screams or that females may also produce Gunshots.

Table 3.5. A table of analysis of video data from SAG observations to determine if the focal female was breathing when Screams and Gunshots were being produced. The table lists the total number of Screams and Gunshots recorded on the video, the total number of breaths observed from the focal female, and the total number of Screams or Gunshots produced simultaneously with a breath from the focal female.

\begin{tabular}{|c|c|c|c|c|c|c|}
\hline Date/SAG & $\begin{array}{c}\text { Duration of } \\
\text { video (minutes) }\end{array}$ & $\begin{array}{l}\text { Number of } \\
\text { breaths from } \\
\text { focal whale }\end{array}$ & $\begin{array}{l}\text { Total Number } \\
\text { of Screams }\end{array}$ & $\begin{array}{l}\text { Total Number } \\
\text { of Gunshots }\end{array}$ & $\begin{array}{l}\text { Screams with } \\
\text { focal breath }\end{array}$ & $\begin{array}{l}\text { Gunshots with } \\
\text { focal breath }\end{array}$ \\
\hline $7 / 15 / 2000$ & 11 & 9 & 16 & 0 & 0 & 0 \\
\hline $8 / 1 / 2000$ & 9 & 6 & 9 & 2 & 0 & 0 \\
\hline $8 / 1 / 2000$ & 20 & 14 & 90 & 27 & 0 & 6 \\
\hline $8 / 1 / 2000$ & 31 & 34 & 83 & 24 & 0 & 7 \\
\hline $8 / 5 / 2000$ & 27 & 10 & 13 & 0 & 0 & 0 \\
\hline $8 / 25 / 2000$ & 12 & 13 & 62 & 11 & 0 & 1 \\
\hline $8 / 25 / 2000$ & 10 & 8 & 20 & 1 & 0 & 0 \\
\hline $9 / 14 / 2000$ & 2 & 3 & 7 & 1 & 0 & 0 \\
\hline $9 / 7 / 2001$ & 12 & 13 & 8 & 1 & 0 & 0 \\
\hline $9 / 7 / 2001$ & 4 & 5 & 3 & 0 & 0 & 0 \\
\hline $8 / 2 / 2002$ & 46 & 27 & 60 & 7 & 0 & 1 \\
\hline $8 / 17 / 2002$ & 42 & 15 & 95 & 6 & 0 & 0 \\
\hline $8 / 24 / 2002$ & 25 & 23 & 55 & 13 & 0 & 3 \\
\hline $8 / 24 / 2002$ & 15 & 10 & 71 & 7 & 0 & 2 \\
\hline $8 / 30 / 2002$ & 4 & 2 & 9 & 2 & 0 & 0 \\
\hline $8 / 30 / 2002$ & 18 & 9 & 98 & 0 & 0 & 0 \\
\hline $8 / 30 / 2002$ & 14 & 3 & 5 & 0 & 0 & 5 \\
\hline $9 / 7 / 2002$ & 20 & 24 & 75 & 14 & 0 & 6 \\
\hline $9 / 17 / 2002$ & 16 & 3 & 19 & 0 & 0 & 0 \\
\hline $9 / 17 / 2002$ & 10 & 1 & 17 & 2 & 0 & 0 \\
\hline Total & 348 & 232 & 815 & 107 & 0 & 31 \\
\hline
\end{tabular}


Results from CART and discriminant analysis of Screams by age/identity of focal female

In a further test of the hypothesis that the focal female is producing the Scream calls, an attempt was made to classify Screams 1) by the age of the focal female and 2) by the individual identity of the focal female. Separation of Screams from adult (greater than 9 years of age or pregnant) and juvenile (1-8 years in age) focal females was modestly successful (Figure 3.18 and Table 3.6). The results in Figure 3.18 show that adult calls tended to be shorter than juvenile calls and have lower frequency amplitude modulation. Separation of Screams from particular individuals (individual labels are listed in Table 3.2) was only slightly more successful than expected by chance with one particular individual's calls separating from the others, though there was a large degree of overlap for most individuals (Figure 3.19 and Table 3.7). CART analysis of individuals show the Acoustat measurements most useful for separating individuals (Figure 3.20).

CART of screams by age of focal

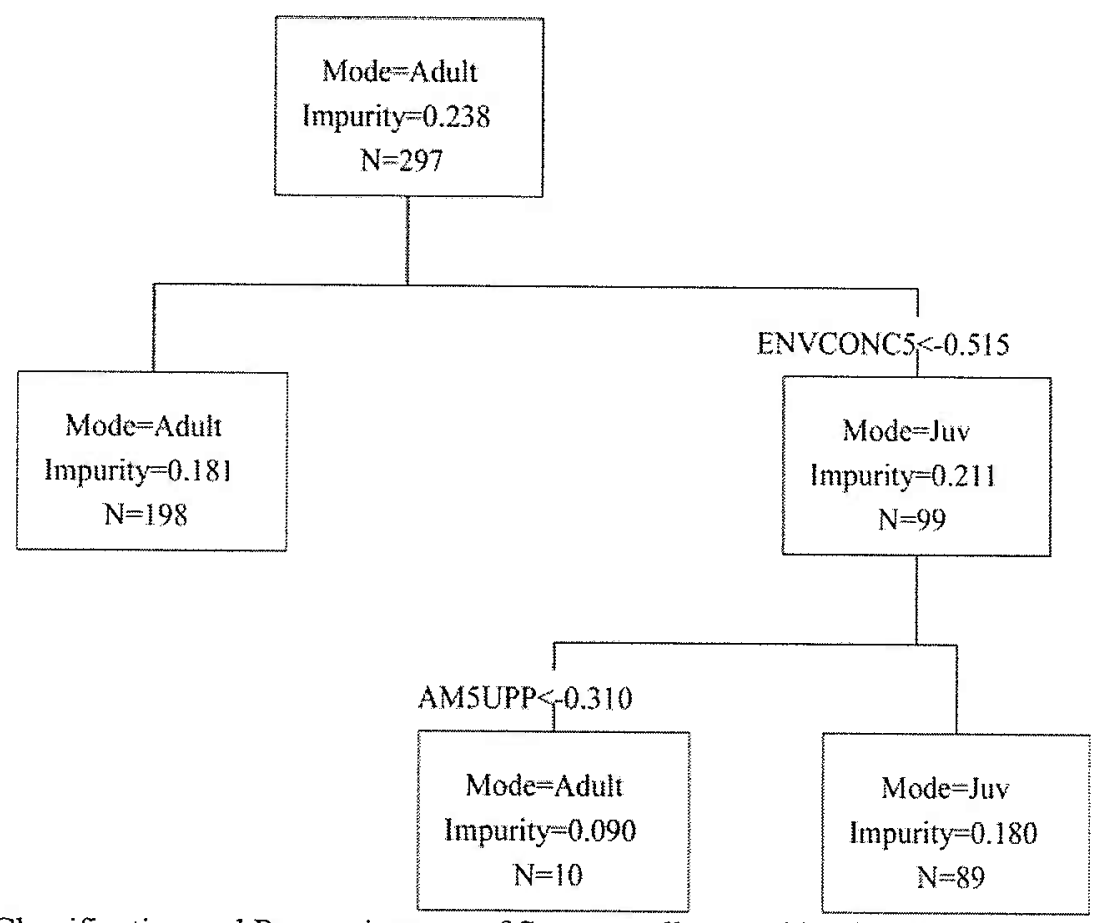

Figure 3.18. Classification and Regression tree of Scream calls sorted by the age of the focal female in the group. The tree was constructed in Systat 10 with a minimum split index value of 0.010 . 
Table 3.6. The jackknifed classification matrix resulting from discriminant analysis between adult and juvenile focal females using all Acoustat variables. For this discriminant analysis the Wilks' lambda $=$ $0.477, \mathrm{~F}=2.467, \mathrm{df}=91,205 \mathrm{p}$-tail $=0.0000$, indicating that there is a significant difference between the two classes.

\begin{tabular}{c|ccc}
\hline & Adult & Juvenile & \%correct \\
\hline Adult & 132 & 49 & 73 \\
Juv & 44 & 72 & 62 \\
Total & 176 & 121 & 69 \\
\hline
\end{tabular}

\section{Canonical Scores Plot}

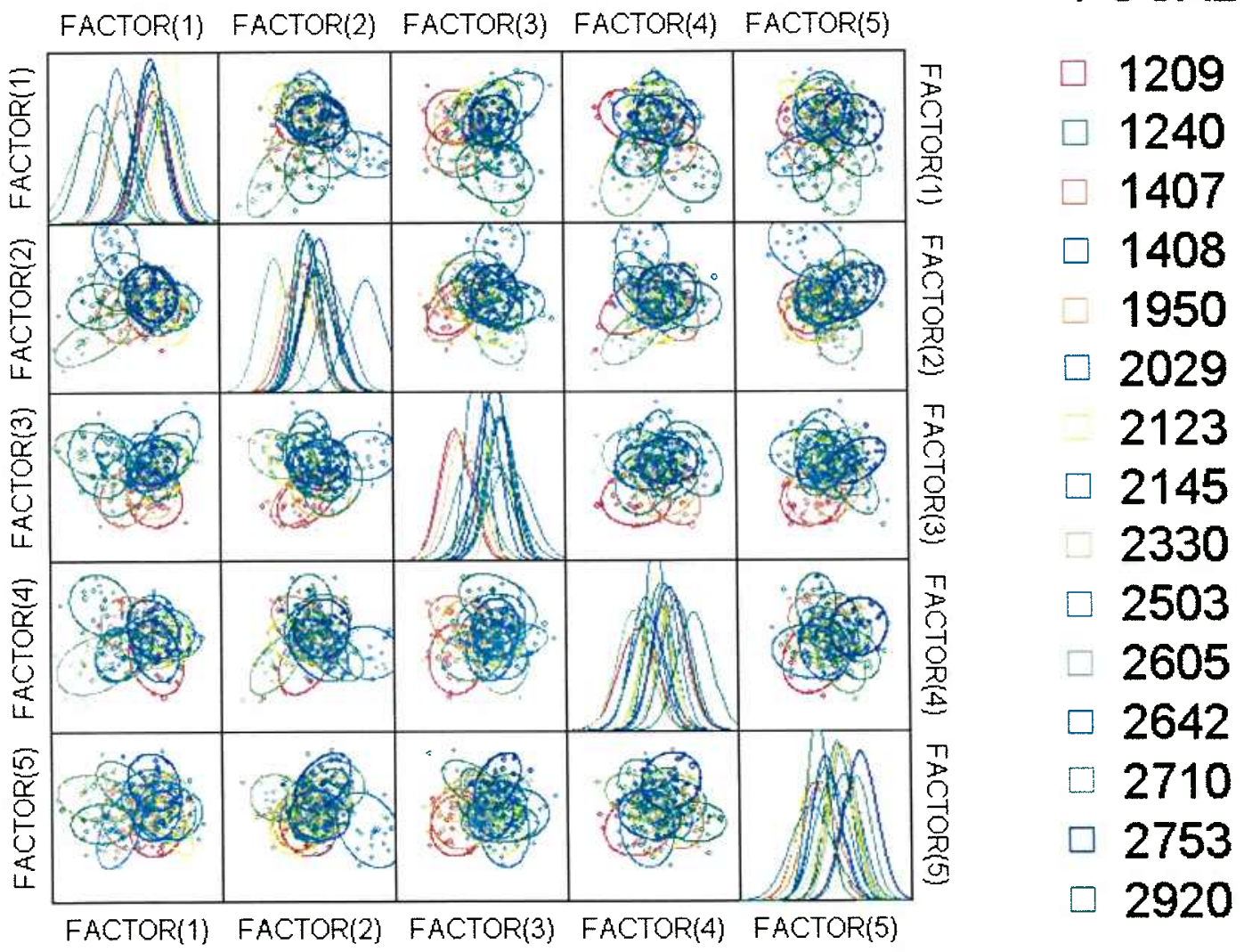

Figure 3.19. Canonical scores plot resulting from discriminant analysis of Screams produced by 15 different individuals. Some individuals were recorded in more than one SAG (see Table 3.2). 


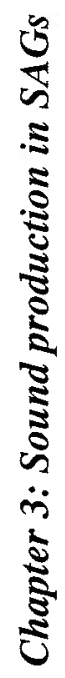

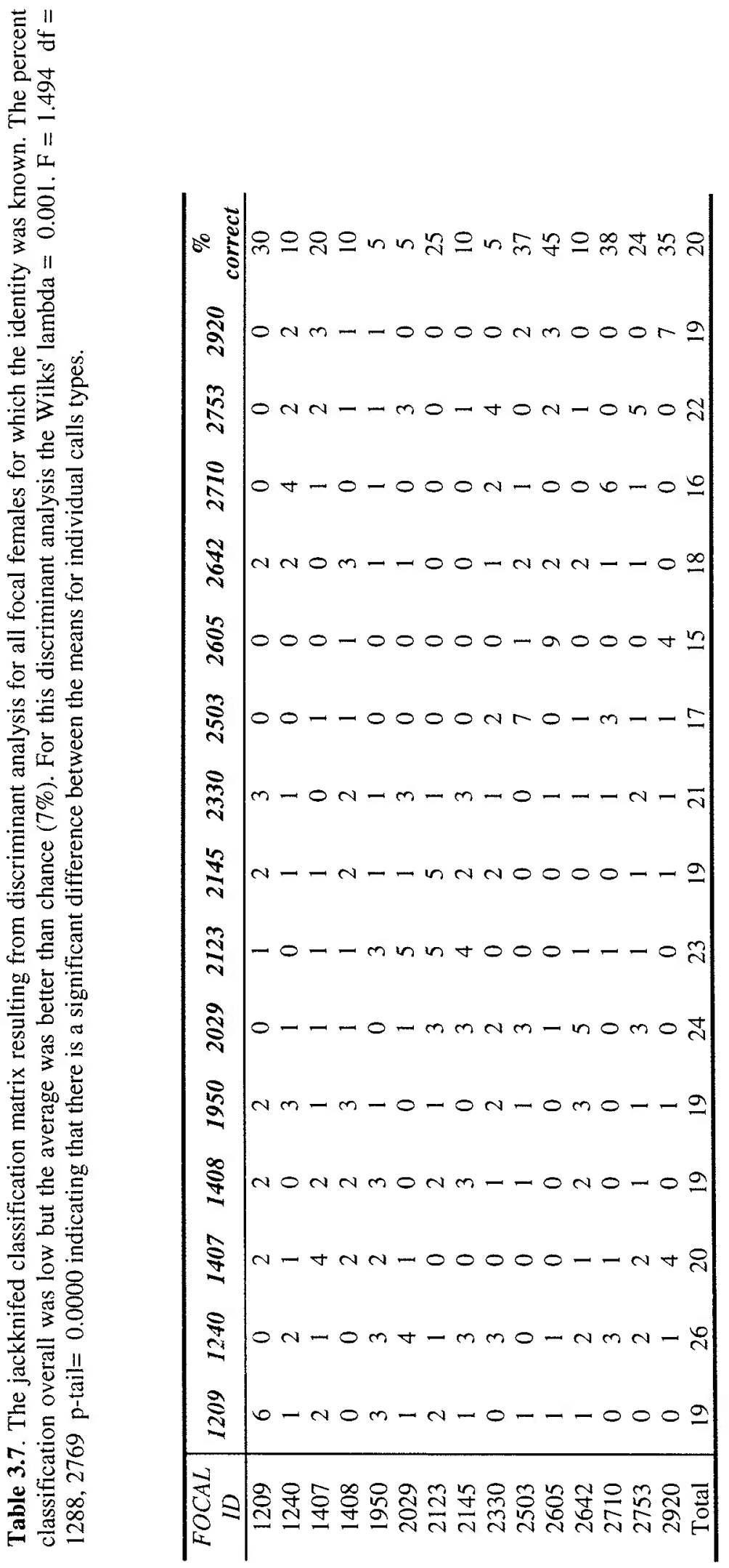




\section{CART of individual focal females}

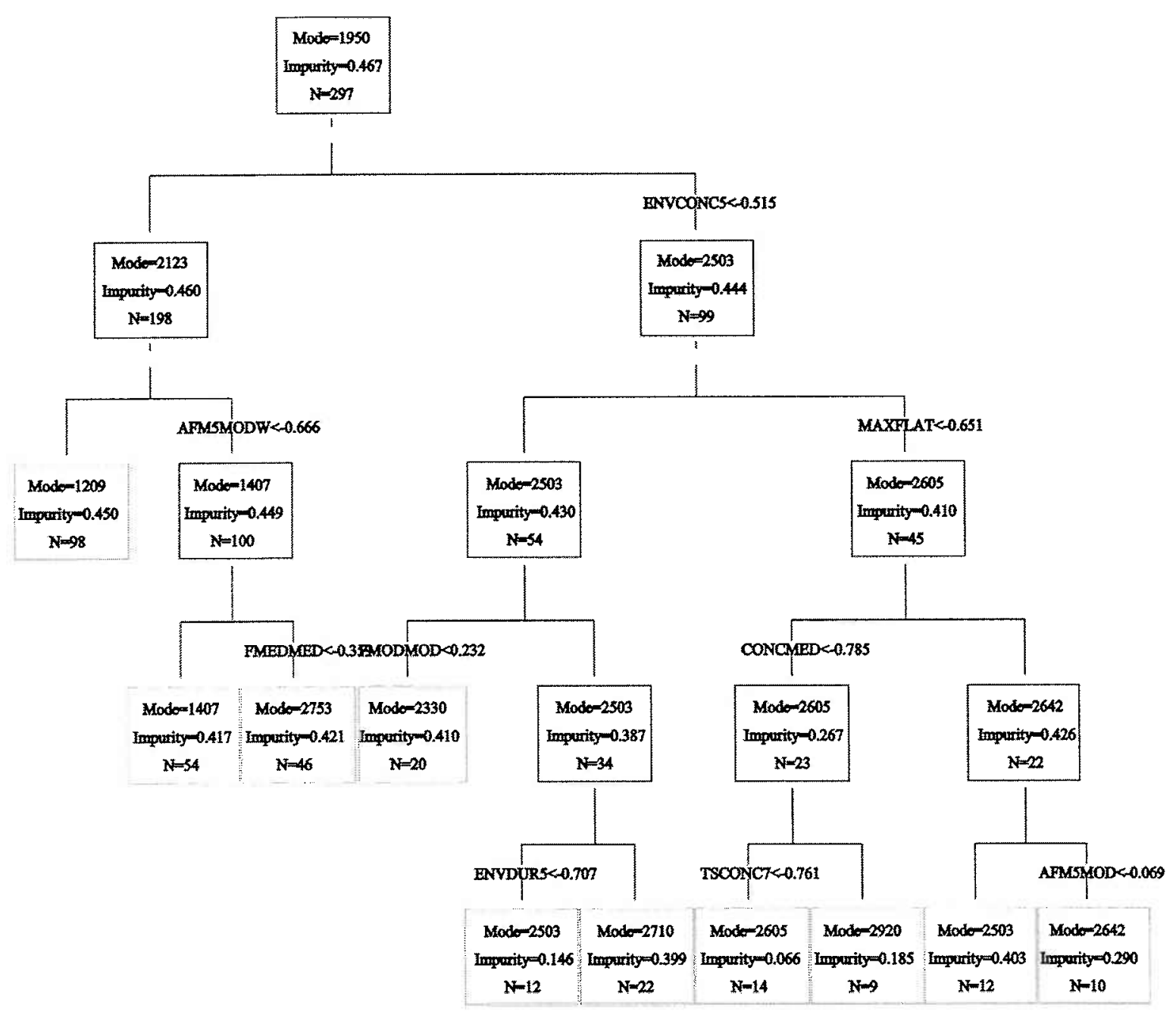

Figure 3.20. CART analysis of Screams by different individual focal females. The tree was constructed in Systat 10 with a minimum split index value of 0.010 . Mode $=$ Individual catalogue ID.

\subsubsection{Source level}

The source level of 30 right whale sounds (22 Screams, 3 Gunshot, 3 Warble and 2 Noisy Blows) was measured. These 30 sounds represent the sounds produced at array bearings consistent with the location of right whales at a known distance. Measurements were made of noise at the same time as the whale sound and within the same frequency range to compare to the measured RL. Sounds were measured from SAGS 35, 36, 40, and 
50 from Table 3.2. Additional noise measurements were taken on 8/1/02, 8/8/02, 8/21/02, $8 / 30 / 02$, and $9 / 2 / 02$. Table 3.8 summarizes the calculated source level measurement for all whale sounds and for whale watching or research vessels near SAGs. Table 3.9 summarizes the measurements of ambient noise conditions. Figure 3.21 shows the distribution of SL for the Scream calls and the noise levels that were measured in both the broadband and $2000-2200 \mathrm{~Hz}$ frequency band. Figure 3.22 shows an example of a right whale Scream and Gunshot sound masked by noise from a research vessel. Figure 3.23 shows the separation between the measured noise level and calculated source level for both sets of measurements. The signal-to-noise ratio was calculated for every calculated source level for the Scream calls (Figure 3.24). The average SNR broadband was $43.8 \mathrm{~dB}$ re $1 \mu \mathrm{Pa}$ for the Scream calls. The average SNR for $2000-2200 \mathrm{~Hz}$ was $51.8 \mathrm{~dB}$ re $1 \mu \mathrm{Pa}$. There was no correlation between broadband SL and NL. There was a weak correlation ( $\mathrm{r}$ $=.379$ ) between the calculated SL and the ambient NL in the $2000-2200 \mathrm{~Hz}$ band (Figure $3.25)$.

Neither simple cylindrical nor spherical spreading would be expected to accurately describe the range for the TL to equal the background noise in the Bay of Fundy, a relatively shallow body of water. A calculation for the range of detection using $20 \log (\mathrm{r})$ to $200 \mathrm{~m}$ and $10 \log (\mathrm{r})$ beyond that range, suggested to describe the transimission loss of right whale sounds in the Bay of Fundy by Hay et al. (Submitted) also gave very short estimates of the range of detectability (154 $\mathrm{m}$ using the broadband SNR and $158 \mathrm{~m}$ using the $2000-2200 \mathrm{~Hz}$ band), barely beyond the range the sounds were originally recorded. SAG calls recorded from up to a $\mathrm{km}$ away are typically audible in recordings, suggesting an error in the equations selected for TL estimates. Whales have been observed approaching these groups from up to $8 \mathrm{~km}$ away (Kraus \& Hatch, 2001). This suggests that TL must be closer to $10 \log (\mathrm{r})$ for which $\mathrm{r}=23 \mathrm{~km}$, or that the whales can detect signals with a SNR < 0 . It is also likely that the higher frequency harmonics are important for long range detection of the groups as they are produced at a higher SNR than the low fundamental frequencies of the calls. 
Table 3.8. a) Calculated SL for 4 sound types and vessel noise all measured as $\mathrm{dB}$ re $1 \mu \mathrm{Pa}$ broadband from $0-20 \mathrm{kHz} . \mathrm{N}=$ number of measurements; $\mathrm{SL}=$ calculated source level. $\mathrm{b}$ ) The same calculated SL for the calls measured as $\mathrm{dB}$ re $1 \mu \mathrm{Pa}$ from $2000-2200 \mathrm{~Hz}$. Gunshot sounds and vessel noise were excluded from these measurements.

a)

\begin{tabular}{c|ccccc}
\hline Broadband & Screams & Warbles & Gunshot & Noisy Blow & Small Vessels \\
\hline N & 22 & 3 & 3 & 2 & 5 \\
Minimum SL & 159.0 & 164.0 & 185.5 & 164.0 & 170.2 \\
Maximum SL & 174.5 & 170.0 & 193.0 & 171.0 & 179.5 \\
Average & 169.2 & 166.0 & 189.2 & 167.5 & 174.1 \\
SD & 4.8 & 3.5 & 3.7 & 4.9 & 4.1 \\
\hline
\end{tabular}

b)

\begin{tabular}{c|ccc}
\hline 2000-2200 Hz & Screams & Warbles & Noisy Blows \\
\hline $\mathrm{N}$ & 22 & 3 & 2 \\
Minimum SL & 121.3 & 135.0 & 120.4 \\
Maximum SL & 148.0 & 140.0 & 142.0 \\
Average & 134.4 & 137.2 & 130.1 \\
SD & 6.4 & 2.6 & 11.0 \\
\hline
\end{tabular}


Table 3.9. The measured noise levels from the Bay of Fundy in summer of 2002. Noise is shown both for broadband and $2000-2200 \mathrm{~Hz}$ frequency ranges.

\begin{tabular}{ccc}
\hline & $\begin{array}{c}\text { Broadband } \\
\text { Noise (RL) }\end{array}$ & $\begin{array}{c}2000-2200 \mathrm{~Hz} \\
\text { Noise (RL) }\end{array}$ \\
\hline $\mathrm{N}$ & 32 & 32 \\
Minimum SL & 120.0 & 75.0 \\
Maximum SL & 132.0 & 89.8 \\
Average & 124.8 & 83.3 \\
SD & 3.2 & 3.6 \\
\hline
\end{tabular}

a)

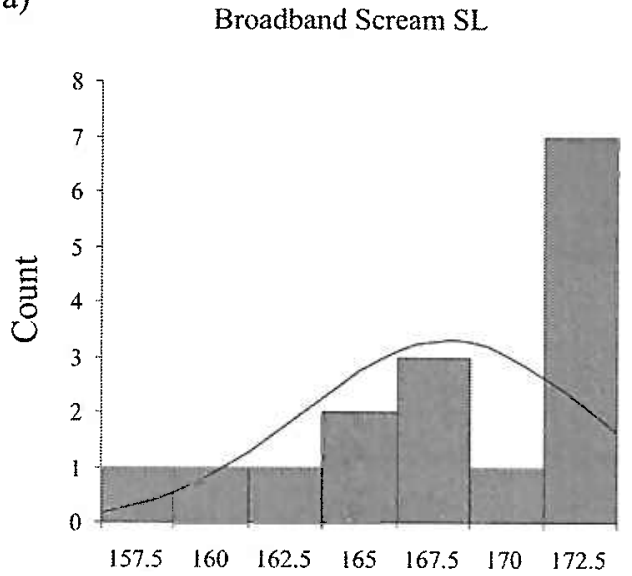

Calculated SL in $\mathrm{dB}$ re $1 \mu \mathrm{Pa}$

c)

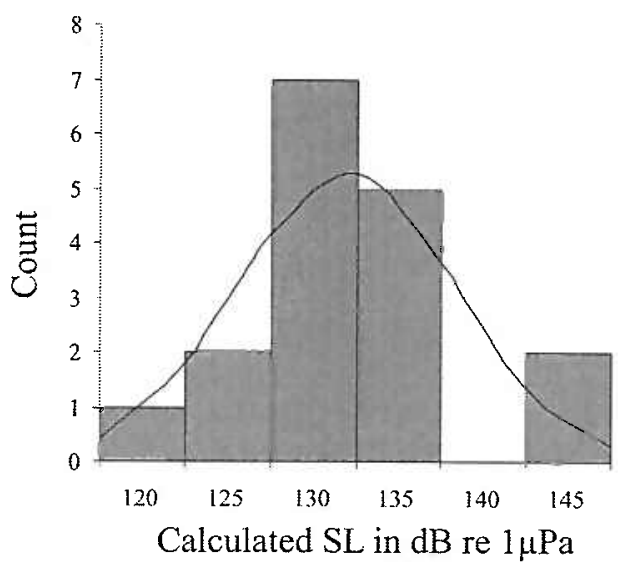

b) Broadband Noise RL

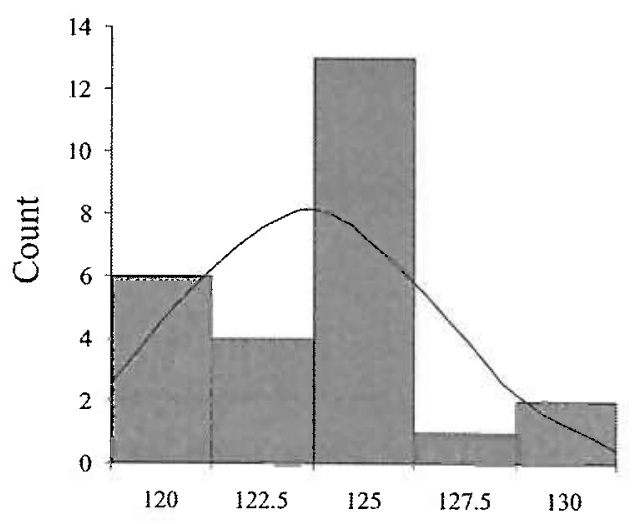

$\mathrm{RL}$ in $\mathrm{dB}$ re $1 \mu \mathrm{Pa}$

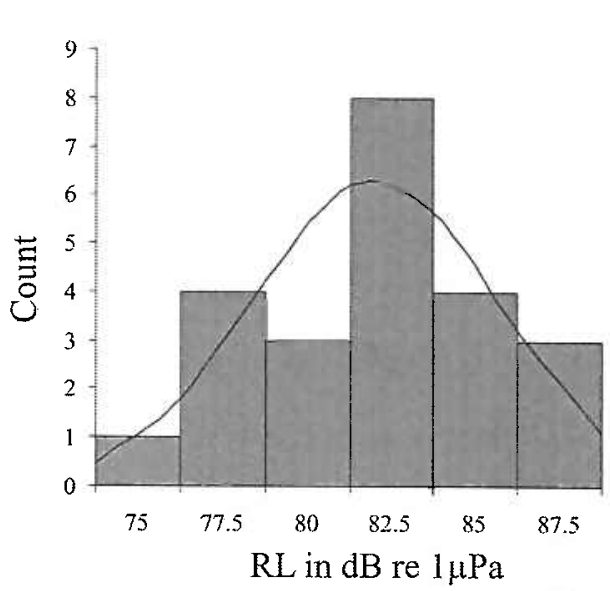

Figure 3.21. a) Distribution of calculated broadband SL for Scream calls b) Measured broadband noise levels c) Calculated $2000-2200 \mathrm{~Hz}$ SL for Scream calls d) Measured noise in the $2000-2200 \mathrm{~Hz}$ band. 


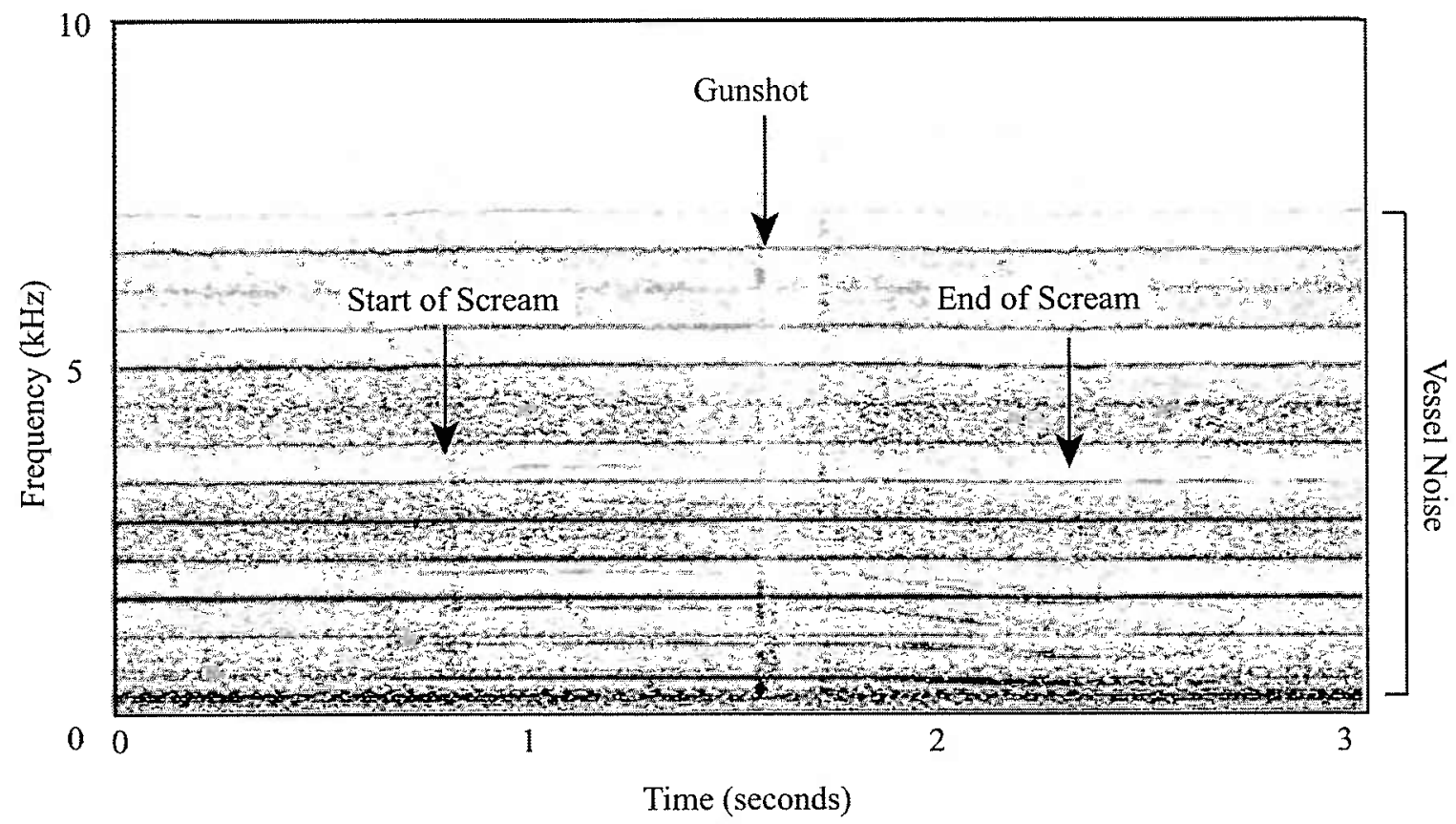

Figure 3.22. An example of a SAG recording made with another research vessel and a SAG approximately $200 \mathrm{~m}$ away. The vessel noise is constant in the figure, with horizontal bands which span approximately $100 \mathrm{~Hz}-7 \mathrm{kHz}$. Note that the Scream and Gunshot sounds produced in the SAG are barely visible on the spectrogram.

a)

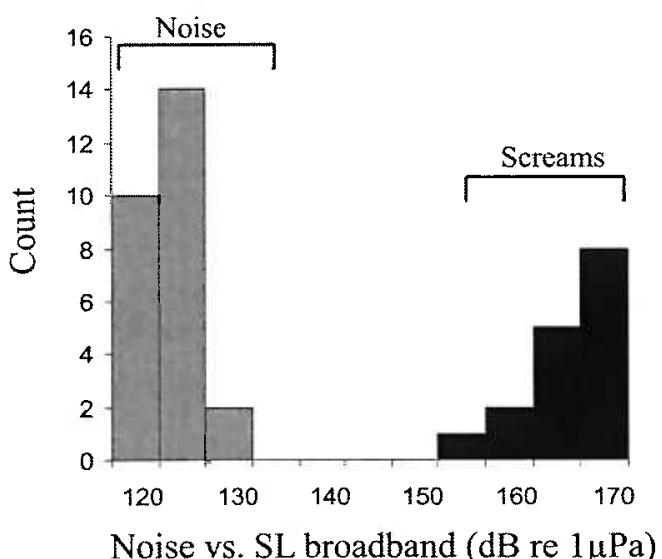

b)

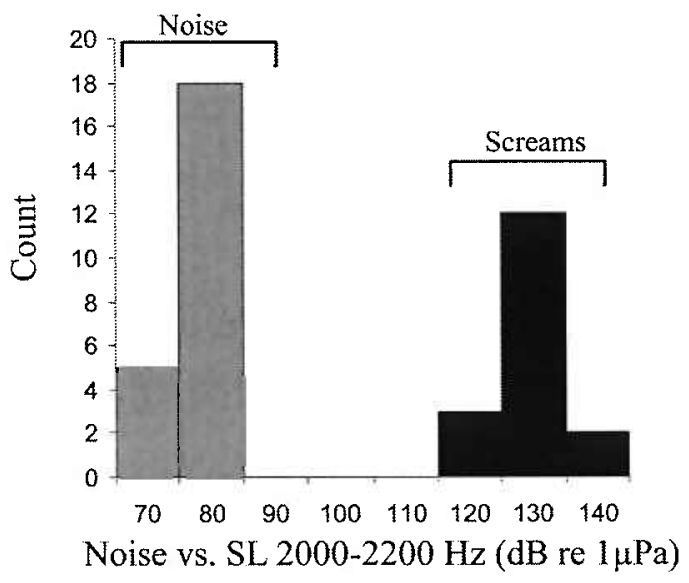

Figure 3.23. Plot showing the measured noise levels and the calculated source levels for the right whale Screams both broadband and in the $2000-2200 \mathrm{~Hz}$ band. These values were used to roughly estimate the range that right whales may be able to detect these signals. 
a)

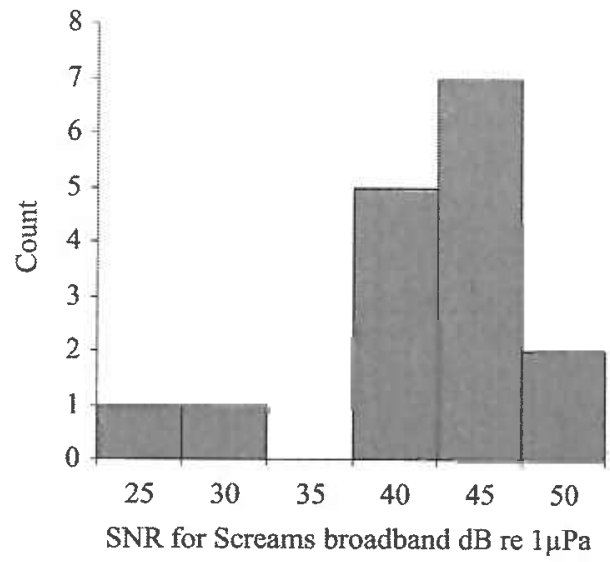

b)

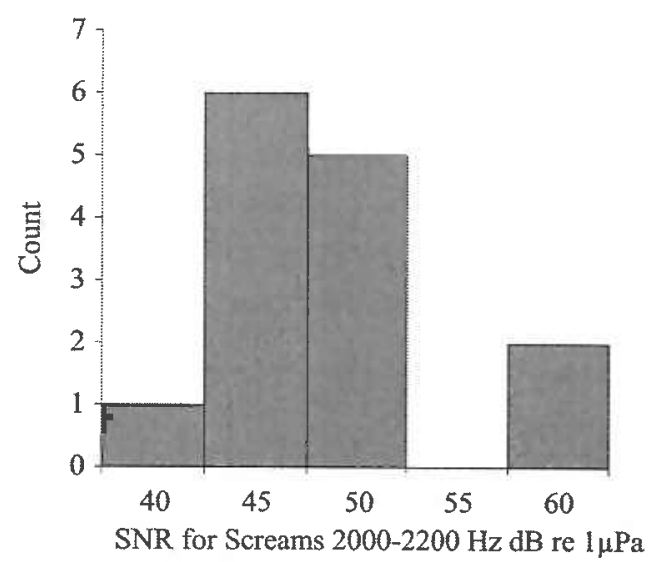

Figure 3.24. Plots showing the signal-to-noise ratio (SNR) for right whale Screams versus the ambient noise level measured in the same frequency band a) Distribution of the broadband SNR b) Distribution of the $2000-2200 \mathrm{~Hz}$ SNR. Note the higher SNR for the $2000-2200 \mathrm{~Hz}$ band.

a)

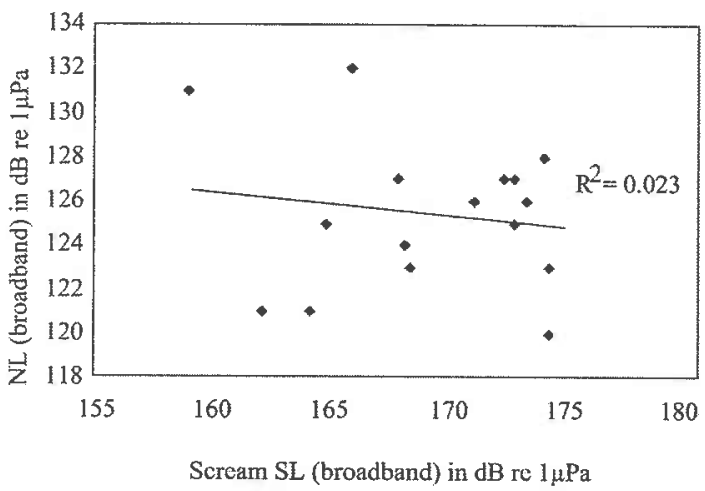

b)

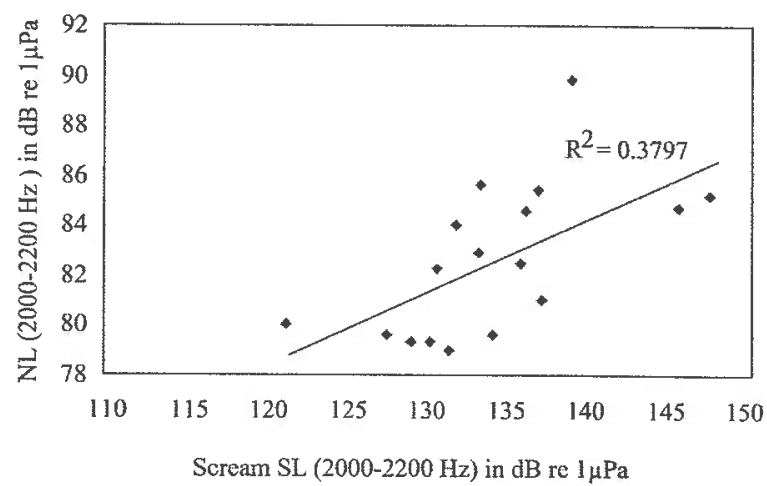

Figure 3.25. Plot showing the calculated SL for the Scream calls versus the measured NL 1 second before the call. There was no apparent correlation between SL and NL in the broadband measurements, but indication of a slight correlation between SL and NL in the $2000-2200 \mathrm{~Hz}$ band.

\subsection{DISCUSSION}

The results presented in this chapter demonstrate that sound production is common in the surface active groups (SAGs) of the North Atlantic right whale. No calls were recorded only in three recording sessions when the SAGs were already breaking up. Analysis of these recordings indicates that several distinct types of sounds were produced 
in the SAGs by several different individuals. Whale sounds were grouped into discrete classes based on previous classification studies of right whale calls (Clark, 1982; Clark, 1983) and for new sounds (e.g. Warble), evidence of different whales producing the sounds. The two dominant types of whale sounds produced in SAGs were the Scream calls and the Gunshot sounds. The recordings made in this study were consistent with previous reports of female production of calls (Kraus \& Hatch 2001). The calls described in Kraus \& Hatch (2001) appear to have been what are described here as Scream calls. However, this chapter demonstrates that other whales in these groups do produce other sounds. Instances of all-male SAGs with only Gunshot sounds being produced, a trend for an increase number of Gunshot sounds to be produced in SAGs with more males present, and the occurrence of overlapping Gunshot sound production in groups with multiple males present indicate that males are responsible for Gunshot sound production. In addition, males and calves associated with SAG produce other sounds, the Upcall and Warble sounds respectively.

\subsubsection{Sound classification}

Principal component analysis and hierarchical cluster analysis yielded results similar to those reported in previous studies repertoire analyses of Southern (Clark, 1982) and North Atlantic right whales (Wright, 2001) with separation of slap, blow and call sounds. The similarity across all three studies occurred even though the measurements made of the sounds were significantly different in both the number of variables and the sound parameters measured. The Acoustat program (Fristrup \& Watkins, 1994), which was not specifically designed for measuring right whale calls was used for this chapter. The similarity across different methods suggests that these categories of sound types are robust categorizations that can be consistently assigned for use in classification. Sound types recorded in these groups were tonal and broadband sounds represented by Screams, Gunshots, Upcalls, Downcalls, Noisy Blow and Warble calls. These sounds correspond to all sound types described for Southern right whales in Patagonia (Clark, 1982) (Table 3). It is possible that much of the North Atlantic right whale repertoire of calls is 
produced in the context of SAG behavior as all sound types described for both Southern and North Atlantic right whales were recorded. However, more recordings of North Atlantic right whales in other behavioral contexts would need to be made to answer this question.

Initial attempts at classifying signals based on Acoustat measurements resulted in mediocre results. CART discriminated Gunshot sounds from all tonal call types. This was not surprising as the Gunshot sounds are distinctly different in all aspects from the tonal calls. Gunshot sounds are very brief, broadband signals with no tonal characteristics. In the CART analysis the duration of the signal was of primary importance in separation of Gunshot sounds from all other sounds (Figure 3.6). The high number of Scream calls dominated the sample, resulting in trees that lumped all tonal signals in with the Scream calls (Figure 3.6). An attempt to improve the discrimination of call types was made reducing the total number of calls compared, and trying to equalize the number of each type represented in the sample. This resulted in CARTs which effectively separated Upcalls, Noisy Blow sounds, Screams and Gunshots (Figure 3.7). The remaining two classes of sounds, Warbles and Down calls, remained unclassified in these trees. Warbles which do not sound extremely different from Screams were included in one of the Scream call branches. The hesitant or quavering quality of the Warble calls that is apparent to a human listener has not been adequately measured to discriminate them from Scream calls. None of the Down calls had a high enough signal-to-noise ratio to be considered for the data set used in the second classification tree.

Two classes of sounds predominate in the SAG recordings, the Scream call and Gunshot sounds. More than $70 \%$ of all sounds recorded from SAGs were the Scream call and the Scream calls were present in recordings of SAGs of all compositions. In studies of Southern right whales, significant differences in the rate of sound production for these sound types were found between active groups and swimming or resting animals (Clark, 1983). The call types recorded in North Atlantic right whale SAGs most closely resemble the sound types documented in fully active and sexually active groups in the Southern right whale (Clark, 1983). 
A notable difference in the sound types produced in North Atlantic right whale SAGs from Southern right whales SAGs is the production of Upcalls. Upcalls were never reported for sexually active groups in the Southern right whale (Clark, 1983). In the North Atlantic, Upcalls were recorded in varying group sizes and in all group types except male-female pairs. Upcalls were never common and made up a very small percentage of the calls recorded for all group types. Several of the groups with Upcalls involved males and females and a male was seen with his penis extended (one of the criteria used by Clark (1983) for definition of sexual activity). The two best explanations for this observed difference would either be seasonal differences (summer feeding grounds in the North Atlantic right whale versus winter calving grounds in the Southern right whale) or species differences in repertoire use.

These results indicate that females produce the Scream calls in SAGs, while males produce the Gunshots and calves produce the Warble sounds. Different classes of sounds were produced in groups with different compositions. Most notably the Warble call was only produced in SAGs with calves present. Array data also suggest that the calf is the source of these calls. Gunshot sounds were produced in the all-male group and while the focal female was breathing in some of the video analyses, indicating that males can produce these sounds. Gunshot sound production has been recorded in three lone males in the Bay of Fundy (Appendix 2) further supporting the hypothesis that males produce Gunshot sounds. All-male groups commonly produced slap sounds in Southern right whales (Clark, 1983), some of which were homologous with Gunshot sounds. These data are all consistent with the hypothesis that males produce the Gunshot sounds in SAGs. Upcalls and Screams are produced by different individuals in the SAGs. Males appear to produce Upcalls in SAGs when joining the group or when the female is on a dive. Females appear to produce the Screams. There is some possibility that males also produce these Scream calls, though it seems unlikely because Scream calls only overlap in timing with other Scream call production when a second or a third female is present in the group. Females may also produce Upcalls and Gunshots in SAGs, but this has yet to be confirmed. 


\subsubsection{Call Timing}

Call timing in SAGs is highly variable. Analysis of timing of the two major sound types, Screams and Gunshots, indicate that there are differences in their rates of production. Both types of sounds appear to be produced in bouts separated by periods of silence. These bouts are not regular in duration within particular SAGs, and the inter-bout periods of silence also vary in duration (Figure 3.9). In the case of Screams, these periods of silence often occur when the focal female is on a dive, even if other whales are still at the surface. The average inter-call interval within a bout are shorter for Screams that for Gunshots. Scream calls are produced much more frequently than Gunshots in all SAGs except the all-male group.

Call rate per minute (CPM) also addresses periods of very high rates of calling that are missed when averaging inter-call intervals over all bouts. These results indicate that higher rates of Gunshot sound production occur in groups with more whales present. However, the same cannot be said for Scream call production, which seems not to vary with group size. Both of these observations are consistent with the hypotheses that Screams are produced by a single focal female and Gunshot sounds are produced by multiple males.

\subsubsection{Individual sound production}

The data on individual sound production adds dimension to the interactions taking place within the SAGs. The publication on SAGs by Kraus and Hatch (2001) gives the impression that the focal female may be the only whale producing sounds in the group. While this study supports the idea that the focal female is responsible for production of the Scream calls, it is clear that other classes of sounds can be produced by other whales in the group. 
Scream calls

The initial hypothesis was that the focal female produced the Scream call in SAGs. Array data collected in 2002 showed that the bearings for all recorded cases of Screams pointed to the SAG. Therefore, whales are not likely producing these calls when they are separated from the group. Unfortunately, no separations of the focal female from the rest of the group of more than 5 degrees were documented during array recordings. It is not possible to clearly assign the Scream calls to only the female from these results. Inspection of focal female behavior in SAGs from video recordings indicates that the female was never seen breathing when a Scream was produced in the group. While the mechanism for sound production in baleen whales is still unknown, it is believed that it involves the respiratory pathway. This would prevent production of all sounds other than Noisy Blows while breathing. Neither the rate of within-bout ICI nor max CPM of Scream calls correlated with the size of a group. Overlapped Scream calls were recorded on two occasions in this study. In both cases, the overlapped Scream calls were heard when two females were present near the center of the group. In both cases, one of the females eventually departed, and no further overlapping of Screams was heard. This adds further support to the idea that female whales produce the Scream calls. Two Screams were recorded during one all-male SAG. This may mean that the broad category of Screams encompasses some sound types that are also produced by males.

\section{Gunshot sounds}

Males were predicted to produce the Gunshot in the SAGs. This hypothesis was based on the observations that lone males produce Gunshot sounds (Appendix 2) and that more Gunshot sounds are produced in groups with more males present. Array data collected in 2002 showed that the bearings for all recorded cases of Gunshots also pointed to the SAG. Whales seemed to produce these sounds primarily while they were interacting directly with other whales. On 31 occasions, the focal female in the group was seen to breath when Gunshot sounds were produced. This indicates that males can 
produce the Gunshot sounds heard in the groups. The max CPM rate for Gunshot sounds increased with increasing group size (Figure 3.14). This suggests that multiple individuals produce these sounds in the groups. These observations do not rule out the possibility of Gunshot sound production by the female, but confirm that males do produce this sound on many occasions in these groups.

Up Calls

Upcalls were recorded in 22 of the 52 SAGs recorded. Even though the percentage of calls represented by Upcalls was always low, Upcalls were commonly detected in the presence of SAGs. Array data in 2002 confirmed that these Upcalls are produced during behaviors related to SAGs. Most notably, the bearings to a male producing Upcalls while swimming into a SAG and bearings to a male left alone at the surface while the focal female was on a dive indicate that males produce these calls. These observations are consistent with recordings and video made from SAGs without the array in the water. On several occasions, loud Upcalls were recorded while males were at the surface and the focal female was on a dive or after the focal female departed the group. The males showed indications that they may be searching for the female during the production of these sounds as they tended to stay in the location of the last position of the SAG but would swim slowly back and forth through the area. Clark (1983) hypothesized that Upcalls function as contact calls in the Southern right whales. It is possible that males use this call to announce their presence to the female. These Upcalls were the only sound recorded when whales approached Scream playbacks in Chapter 4.

Warbles

Warble sounds had not been previously described for North Atlantic right whales. Warble sounds were only recorded when calves were present in the SAG and therefore it was predicted that calves were responsible for producing these sounds. Array bearings in 2002 provided evidence that the calves produce this sound as good bearings were 
obtained to a calf alone at the surface while Warble sounds were being produced and all other whales in the SAG were down on a dive. These Warble sounds were only recorded in two SAGs. In one SAG in 2001 when Warbles were recorded, the calf was female. The gender of the calf in the 2002 SAG is still unknown but it is possible that only female calves will produce these Warble sounds. These sounds may represent practice on the part of a female calf. It remains to be seen if all the calves in SAGs that had no documented Warble sounds were males. Perhaps the first summer the mothers intentionally form SAGs to teach their calves about the groups. Both male and female offspring would benefit from this experience.

\section{Individual \& Age discrimination}

Right whales may form long term social relationships that are based on the ability to use sound to stay in contact. The high variability of Scream calls from initial classification led me to predict that individual recognition might be possible based on this call type. Attempts were made to control for recording conditions by using recordings from more than one SAG with the same female present as the focal. Therefore, if there were groupings based on individual, it would not be a result of recording session, but rather on some more stable difference in call characteristics between different individuals.

Eight female whales (Table 3.2) were recorded on two occasions in different SAGs. The remaining seven females were only recorded on one occasion. Pooling all Screams recorded from particular individuals and then attempting to separate them, both by CART and by discriminant analysis was not hugely successful with accuracy of classification ranging from $5-45 \%$ correct classification. There was a significant difference between the mean values of Scream call variables by individual $(\mathrm{P}<.0001)$. Future attempts at discrimination using spectrogram cross correlation may yield better results (Cortopassi \& Bradbury, 2000) although the high variability of this call type may not be amenable to comparing calls by spectrogram cross-correlation methods. 
Classification of Scream calls by the age of the focal female was made with CART and discriminant analyses. Screams produced by adults were discriminated from Screams produced by juveniles with reasonable success from the entire data set, though there was substantial overlap. This suggests that a difference may exist between juvenile and adult calls in these groups, though separation of calls produced by age, rather than two categorical groups (Juvenile or Adult) may help to describe these differences more fully. Males may very well be able to distinguish between juvenile and adult females, leading to differences in group size, composition, and activity level.

\subsubsection{Source level (SL)}

The broadband source level of the sounds produced by North Atlantic right whales in SAGs here are in agreement with previous reports of source level for Southern and North Atlantic right whales (Richardson et al., 1995; Hay et al., Submitted). The received level (RL) for these signals was often very similar to the ambient noise levels in the same frequency band even though the signals were clearly audible. Perhaps the range of frequencies chosen to quantify the SL could be improved, by looking at $1 / 3$ octave bands around the peak energy spectrum of the calls. The recorded noise levels in the Bay are consistent with noise levels reported for areas with moderate to heavy shipping traffic (Wenz, 1962). It is possible that these higher noise levels limit the range that right whales are capable of communicating in these groups. However, it seems likely that the whales are capable of producing higher source levels (as seen in the variability of measured SL) that would be able to compensate for the ambient noise levels. The results here show little evidence for whales increasing the overall source level at times of higher noise. However, the whales may be able to increase the energy of the signals at higher frequencies. This would increase the range of detectability because noise levels are generally lower at higher frequencies. Masking from whale watching and research vessels should be considered carefully because the peak noise level and the peak right whale signal level overlap considerably in these frequencies, and the calculated source levels for the 
research and whale watch vessels is approximately $5-10 \mathrm{~dB}$ higher than the source level of the whales themselves (Table 3.8).

\subsubsection{Possible improvements to be considered for future studies}

Many of the results in this chapter can provide the basis for future studies. Some experiences gained in the collection of the data may improve future work. A dedicated platform is essential for long-duration observations of these groups. A larger platform or an aerostat would provide better height for observation. The perspective of the group changes dramatically from low (such as the R/V Bonita RHIB), mid (R/V Stellwagen) or high (video from DFO aerostat (Harris \& Hain, 2002)) elevation above the water. This change in perspective allows for better separation of different individuals in the group and permits some observations of whales underwater. In many of my array recordings, it was impossible to tell whether other whales were present just below the surface. The "stationary" deployment of the array for observation worked reasonably well. However, the platform was never stationary during recording, always being pushed by current and/or wind slowly away from the SAG. This prevented the array from changing orientation because it had tension on the lines attached to the vessel which maintained its horizontal/parallel arrangement. Frequent (approximately every 20-30 minutes) repositioning was required, but I felt that this was far superior, both in terms of observations and minimizing disturbance to the whales, than constant circling of the group with the engine running. On one occasion when there was no wind at mid-tide, deployment of the array resulted in such poor orientation that it could not be used to collect accurate bearing data. The array could be seen from the surface on that day, and it could be seen rotating around its center point because there was no tension on the lines supporting it, allowing it to twist beneath the vessel. The ability to do beam-forming in the field while collecting behavioral information is extremely valuable. If a larger crew was used it may have been possible to collect very good bearing data from the array and visual observations to the whales when every call was produced. Overall, a dedicated focused research effort could add valuable data to this initial study. 


\subsection{Conclusions}

The goal of this chapter was to characterize the acoustic activity of North Atlantic right whales in SAGs. It is hoped that complete characterization of the sounds produced in SAGs, the timing of production and the identification of individuals producing the sound will have multiple applications. Females produce Scream calls, Males produce Gunshot sounds, and calves produce Warbles. It is clear that except for the Warble call, there is no diagnostic sound type for different SAG compositions. However, the proportion that each sound type contributes to the total acoustic signature is significant. It is possible that these different types of sounds represent different motivations on the part of individual whales. For example, if Gunshot sounds represent agonistic interactions between males, it is not surprising that a larger proportion of these sounds are heard when no females are present, or when more males are present in a single group.

It is hoped that these data will be useful both to behavioral research and conservation efforts. In behavioral research the use of acoustics can aid in discrimination between broadly similar observed behaviors termed SAGs. Acoustic descriptions of SAGs will be particularly useful for informing the design of passive acoustic detection systems to account for the behavior and total number of animals that may be present.

\subsection{LITERATURE CITED}

Bradbury, J. W. \& Vehrencamp, S. L. 1998. Principles of animal communication. Sunderland, MA: Sinauer Associates, Inc.

Breiman, L. 1984. Classification and regression trees. Belmont, Calf.: Wadsworth International group.

Charif, R. A., Mitchell, S. \& Clark, C. W. 1995. Canary 1.2 User's Manual. Ithaca, NY: Cornell Laboratory of Ornithology. 
Cheney, D. L. \& Seyfarth, R. M. 1988. Assessment of meaning and the detection of unreliable signals by vervet monkeys. Animal Behaviour, 36, 477-486.

Clark, C. W. 1982. The acoustic repertoire of the southern right whale, a quantitative analysis. Animal Behavior, 30, 1060-1071.

Clark, C. W. 1983. Acoustic communication and behavior of the southern right whale. In: Behavior and communication in whales. American Association for the Advancement of Science Selected Symposium 76 (Ed. by Payne, R. S.), pp. 163198. Boulder, CO.: Westview Press.

Clark, C. W. 1984. Acoustic communication and behavior of southern right whales, Eubalaena australis. National Geographic Society Research Reports, 17, 897907.

Clark, C. W. 1999. The application of autonomous seafloor acoustic recorders for monitoring right whales. In: North Atlantic Right Whale Consortium Meeting (Ed. by M.Marx). Boston, MA.

Clark, C. W. \& Clark, J. M. 1980. Sound playback experiments with southern right whales (Eubalaena australis). Science, 207, 663-665.

Clark, C. W. \& Fristrup, K. M. 1997. Whales '95: A combined visual and acoustic survey of blue and fin whales off southern California. Rep. Int. Whal. Commn., 47, 583600 .

Cortopassi, K. A. \& Bradbury, J. W. 2000. The comparison of harmonically rich sounds using spectrographic cross-correlation and principal coordinates analysis. Bioacoustics, 11, 89-127.

Cummings, W. C., J. Fish, P. Thompson. 1972. Sound production and other behavior of southern right whales, Eubalaena glacialis. Transactions of the San Diego Society of Natural History, 17, 1-14.

Deecke, V. B., Ford, J. K. B. \& Spoong, P. 1999. Quantifying complex patterns of bioacoustic variation: Use of a neural network to compare killer whale (Orcinus orca) dialects. Journal of the Acoustical Society of America, 105, 2499-2507.

Desharnais, F., Laurinolli, M., Hay, A. \& Theriault, J. A. 2000. A scenario for right whale detection in the Bay of Fundy. In: Oceans 2000, pp. 1735-1742. Providence, RI.

Devore, J. L. 1995. Probability and statistics for engineering and the sciences. Pacific Grove, CA: Duxbury Press. 
D'Vincent, C. G., Nilson, R. M. \& Hanna, R. E. 1985. Vocalization and coordinated feeding behavior of the humpback whale in southeastern Alaska. Scientific Report of the Whales Research Institute, Tokyo, 36.

Fristrup, K. M. \& Watkins, W. A. 1994. Marine animal sound classification. pp. 29. Woods Hole, MA: Woods Hole Oceanographic Institution.

Harris, L. E. \& Hain, J. 2002. Cetacean observations from an aerostat: a developing capability. In: North Atlantic Right Whale Consortium Meeting (Ed. by M.Marx). New Bedford, MA.

Hay, A. E., Laurinolli, M. H., Desharnais, F. \& Schillinger, D. J. Submitted. Source levels of North Atlantic right whale sounds in the Bay of Fundy. Journal of the Acoustical Society of America.

Helweg, D. A., Frankel, A. S., Mobley, J. R. \& Herman, L. M. 1992. Humpback whale song: our current understanding. In: Marine Mammal Sensory Systems (Ed. by Thomas, J. A., Kastelein, R. A. \& Supin, A. Y.). New York: Plenum Press.

Hopp, S. L. \& Morton, E. S. 1998. Sound playback studies. In: Animal Acoustic Communications: Sound Analysis and Research Methods (Ed. by Hopp, S. L., Owen, M. J. \& Evans, C. S.). Berlin: Springer.

Howard, R. D. \& Young, J. R. 1998. Individual variation in male vocal traits and female mating preferences in Bufo americanus. Animal Behaviour, 55, 1165-1179.

IFAW. 2001. Report of the workshop on right whale acoustics: practical application in conservation. (Ed. by Gillespie, D. \& Leaper, R.), pp. 23. Yarmouth Port, MA: International Fund for Animal Welfare.

Janik, V. M. 1999. Pitfalls in the categorization of behaviour: a comparison of dolphin whistle classification methods. Animal behaviour, 57, 133-143.

Janik, V. M. \& Slater, P. J. B. 1998. Context-specific use suggests that bottlenose dolphin signature whistles are cohesion calls. Animal Behaviour, 56, 829-838.

Knowlton, A. R. \& Kraus, S. D. 2001. Mortality and serious injury of northern right whales (Eubalaena glacialis) in the western North Atlantic. Journal of Cetacean Research Management (Special Issue) 2, 193-208.

Kraus, S. D. \& Hatch, J. J. 2001. Mating strategies in the North Atlantic right whale (Eubalaena glacialis). The Journal of Cetacean Research and Management (Special issue) 2, 237-244. 
Martin, P \& Bateson, P. 1993. Measuring Behaviour: An introductory guide. Cambridge: Cambridge University Press.

Matthews, J. N., Brown, S., Gillespie, D., Johnson, M., McLanaghan, R., Moscrop, A., Nowacek, D., Leaper, R., Lewis, T. \& Tyack, P. 2001. Vocalisation rates of the North Atlantic right whale (Eubalaena glacialis). Journal of Cetacean Research \& Management, 3, 271-282.

Miller, P. J. \& Tyack, P. L. 1998. A small towed beamforming array to identify vocalizing resident killer whales (Orcinus orca) concurrent with focal behavioral observations. Deep-Sea Research, 45, 1389-1405.

Payne, R. S. \& McVay, S. 1971. Songs of humpback whales. Science, 173, 585-597.

Payne, R. S. \& Payne, K. 1971. Underwater sounds of southern right whales. Zoologica, 58, 159-165.

Potter, J. R., Mellinger, D. K. \& Clark, C. W. 1994. Marine mammal call discrimination using artificial neural networks. Journal of the Acoustical Society of America, 96, 1255-1262.

Rendall, D., Rodman, P. S. \& Emond, R. E. 1996. Vocal recognition of individuals and kin in free-ranging rhesus monkeys. Animal Behaviour, 51, 1007-1015.

Richardson, W. J., Greene Jr., C. R., Malme, C. I. \& Thomson, D. H. 1995. Marine mammals and noise. San Diego: Academic Press.

Saayman, G. S. \& Tayler, C. K. 1973. Some behavior patterns of the southern right whale, Eubalaena australis. Sonderdruck aus Z. F. Saugetierkunde Bd., 38, 172183.

Schevill, W. E., Backus, R. H. \& Hersey, J. B. 1962. Sound production by marine animals. In: Bioacoustics (Ed. by Hill, M. N.), pp. 540-566. New York: Wiley.

Schevill, W. E. \& Watkins, W. A. 1962. Whale and porpoise voices: a phonograph record. pp. 24. Woods Hole, MA: Woods Hole Oceanographic Institution.

Silber, G. K. 1986. The relationship of social vocalizations to surface behavior and aggression in the Hawaiian humpback whale (Megaptera novaeangliae). Canadian Journal of Zoology, 64, 2075-2080.

Slater, P. J. B. \& Lester, N. P. 1982. Minimising errors in splitting behaviour into bouts. Behaviour, 79, 153-161.

Tabachnick, B. G. \& Fidell, L. S. 1983. Using multivariate statistics. New York: Harper \& Row Publishers. 
Tyack, P. \& Whitehead, H. 1983. Male competition in large groups of wintering humpback whales. Behaviour, 83, 132-154.

Urick, R. J. 1983. Principles of underwater sound. Los Altos, CA: Peninsula Publishing.

Vanderlaan, A. S. M., Hay, A. E. \& Taggart, C. T. 2000. Quantitative characterisation of North Atlantic right whale (Eubalaena glacialis) vocalisations in the Bay of Fundy. In: Right Whale Consortium Meeting (Ed. by Marx, M.). Boston, MA.

Wenz, G. M. 1962. Acoustic ambient noise in the ocean: spectra and sources. Journal of the Acoustical Society of America, 34, 1936-1956.

Winn, H. E. \& Winn, L. K. 1978. The song of the humpback whale Megaptera novaeangliae in the West Indies. Marine Biology, 47, 97-114.

Wright, D. R. 2001. Categorization of northern right whale, Eubalaena glacialis, sound. M.A. Thesis. Boston University, Boston,MA. 


\title{
CHAPTER 4. PLAYBACK EXPERIMENTS TO NORTH ATLANTIC RIGHT WHALES IN THE BAY OF FUNDY
}

\author{
Data reprinted with permission from Marine Mammal Science
}

\subsection{INTRODUCTION}

The North Atlantic right whale (Eubalaena glacialis) population migrates along the east coast of the United States from northern waters in the Gulf of Maine in the spring and summer to the waters off the states of Florida and Georgia in the winter (Winn et al., 1986). Despite protection from whaling for the past 60 years, current estimates indicate that fewer than 300 North Atlantic right whales remain (Knowlton et al., 1994; IWC, 2001), making them one of the most endangered large whales in the world. All individuals in the population can be identified based on individually distinctive external features, such as callosity patterns on the head or scars on the body and tail (Kraus et al., 1986). Long-term studies relying on photographic identification of individuals have provided data on many life history parameters for individuals in the population and reproductive history for all females born since 1980 (Kraus et al., 2001; Brown et al., 1994). Demographic information this detailed is rare for a baleen whale species; it enables studies of behavioral interactions among known individuals in a wild population. Each summer up to $2 / 3$ of the known population of North Atlantic right whales congregate in the Bay of Fundy, Canada to feed (23-199 individuals from 1980-1998 (IWC 2001) with 173, 154 and 123 for 1999, 2000, and 2001 respectively (Right Whale Consortium data catalog $\left.{ }^{1}\right)$ ). The high concentration of known individuals in a small area makes this an ideal location to study social behavior.

Little is known about reproductive behavior in the right whale or any other baleen whale species. Observations of social groups in several species of coastal baleen whale indicate possible similarities in social and sexual behavior. In particular, distinct sounds have been associated with surface active groups in North Atlantic right, South Atlantic

\footnotetext{
${ }^{1}$ Right Whale Consortium data catalog is maintained by the New England Aquarium, Right Whale Research Group, Boston, MA.
} 
right (Eubalaena australis), bowhead (Balaena mysticetus), gray (Eschrichtius robustus), and humpback (Megaptera novaeangliae) whales (Clark, 1983; Norris et al., 1983;

Silber, 1986; Würsig \& Clark, 1993; Kraus \& Hatch, 2001).

Male right whales have disproportionately large testes $(900+\mathrm{kg}:(\mathrm{Om} u \mathrm{ra}$ et al., 1969)): the largest for any mammal and the largest as a percentage of body weight for any baleen whale (Brownell \& Ralls, 1986). Large testis size has been linked to sperm competition in a variety of mammalian species (e.g. (Harcourt et al., 1981; Kenagy \& Trombulak, 1986; Møller, 1989)), suggesting that sperm competition plays a role in right whale reproduction (Brownell, 1986). One of the most obvious social groupings of North Atlantic right whales, surface active groups (SAGs), often involves a single adult female, referred to as the focal female, surrounded by up to 34 males maneuvering to approach the female (Kraus \& Hatch, 2001). Observations of behavior in the SAGs support the theory for sperm competition, as the interactions between males in the groups are generally only mildly aggressive and multiple males have been observed to achieve intromission with the same female in these groups (Brownell, 1986). Adult males appear to be highly motivated to join these groups and can detect and locate them from great distances. Male right whales have been observed joining SAGs by swimming rapidly from up to $8 \mathrm{~km}$ away at speeds up to $15 \mathrm{~km} / \mathrm{hr}$ (Kraus \& Hatch, 2001). The adult sex ratio of this population is close to $1: 1$ (Brown et al., 1994), but the calving interval for females is approximately 4 years, so there are potentially four adult males for every receptive female during the mating season. The inter-calf interval in this population has increased from an average of 3.7 years during 1980-1992 to over 5 years during 19931998 (Kraus et al., 2001), further widening the sex ratio between sexually receptive females and males. All of these characteristics suggest that sperm competition and possibly female incitation of male-male competition may characterize the reproduction in this endangered species.

Particular calls are associated with surface active groups (SAGs) in North Atlantic right whales. The types of sounds recorded include Scream calls, Gunshot sounds, Blow sounds, Upcalls, Warbles and Downcalls (Chapter 3). An example of sounds recorded 
from a SAG in the Bay of Fundy in 2000 is shown in Figure 4.1. These bouts of high rates of calling are typically broken up by periods of silence, often associated with the entire group diving beneath the surface for periods of 10 minutes or more. It is likely that approaching whales use the sounds produced in these groups to locate them. Indirect evidence suggests that the Scream calls, the major type of sound produced by right whales in SAGs, are produced by the focal female to attract males, thereby increasing the number of males competing for access to the female. The evidence for this includes: on all recordings of SAGs in which only a single female was present, single Screams were only heard alone and never simultaneously, no instances of Screams being made while the focal female whale was breathing, and the cessation of Scream production with the departure of the focal female (Kraus \& Hatch, 2001). Screams are thought to be produced by only one individual within each SAG and the fundamental frequency, duration and modulation of individual calls are highly variable (Fig 4.1b-c).

Observational evidence (Chapter 3) suggests that male North Atlantic right whales produce Gunshot-like sounds, so named because they sound like a gunshot. This sound has yet to be recorded from female North Atlantic right whales. Little background information exists for this behavior in right whales. Gunshot sounds, characterized by a very short intense broadband cracking sound usually followed by a strong echo off the bottom in the Bay of Fundy (Fig. 4.1d), have been recorded in SAGs and from lone male animals in the population (Appendix 2). In SAGs, multiple animals appear to be making Gunshot sounds as they overlap calls and/or other Gunshot sounds. These sounds are produced by whales without any visible flipper or fluke movement and they are acoustically distinct from flipper slaps, lobtailing or breaching suggesting an internal mechanism for production. A similar sound has been documented both in southern right whales and bowhead whales (Clark, 1983; Würsig \& Clark, 1993) and it has been suggested that the sound is produced in agonistic displays (Clark, 1983). Gunshot sounds produced by lone animals in the Bay of Fundy do not appear to attract any other right whales. Recordings of Gunshots being produced by lone males $(n=3)$, coupled with the observation of Gunshot sounds in surface active groups with multiple males competing 
for access to a centralized female $(n=31)$, suggest that the Gunshot sound functions either as an agonistic display, a male advertisement display for female choice, or both. Unlike Scream calls, which have only been recorded in SAGs, Gunshot sounds are produced during two very different social states (lone males vs. whales in a SAG), therefore these sounds should be a less reliable cue that a SAG is taking place.

Playback experiments have been used to study many different aspects of communication and behavior in marine mammals over the past three decades, including playbacks of natural sounds to Southern right whales (Clark \& Clark, 1980), humpbacks (Tyack, 1983; Frankel, 1995), and gray whales (Cummings \& Thompson, 1971). Playbacks of sounds recorded from SAGs to North Atlantic right whales make it possible to confirm that the sounds in the groups attract other whales. Sexually mature males appear to be highly motivated to approach SAGs with females and are predicted to approach any sound that might indicate the presence of such a group. Females are predicted to not approach playbacks of Scream calls. It is possible that males locate these groups by detecting a variety of sounds associated with SAGs, such as Screams, Gunshot sounds, Upcalls and loud, audible underwater blow sounds. The purpose of this experiment was to determine if the Scream calls produced in SAGs are sufficient to attract males to these groups without additional acoustic cues. To test if this is the case, playback trials using only Screams from SAGs or only Gunshot sounds were conducted. Gunshot sounds are produced both in SAGs and by lone animals, so the Gunshot playbacks were designed to determine if right whales would approach any sound associated with a SAG, even one that is not a reliable indicator of a SAG. If the Scream calls are the primary cue which adult males use to detect a SAG, few or no animals would be expected to approach the Gunshot sound playbacks. 

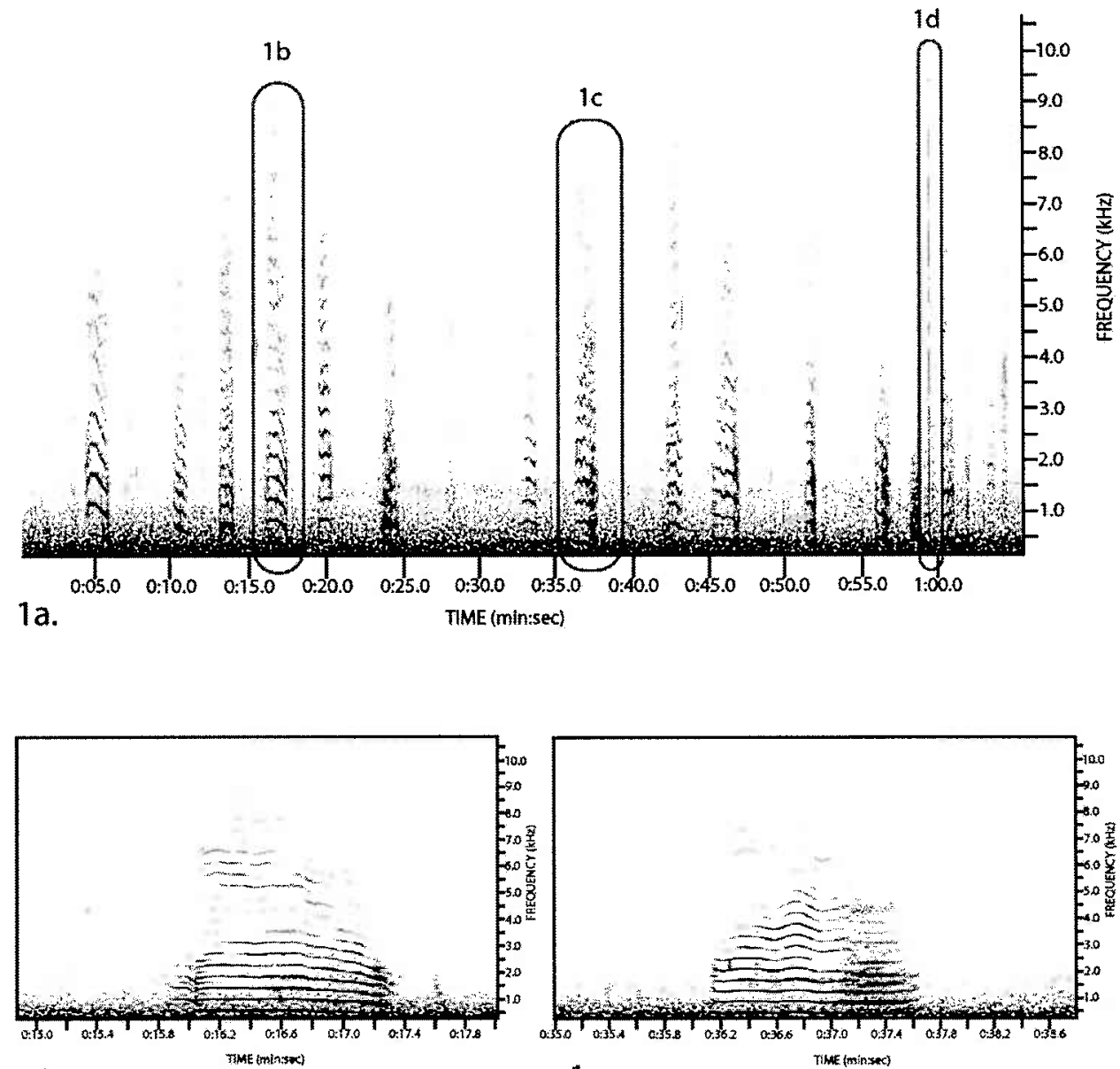

$1 b$.

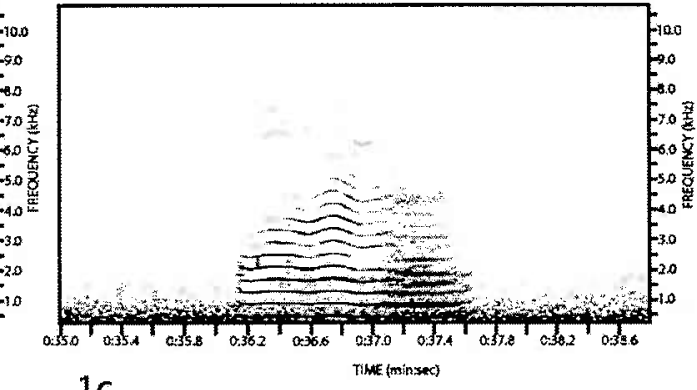

$1 c$.

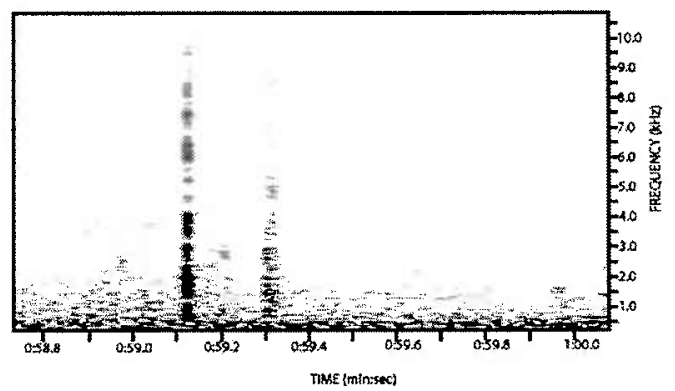

$1 d$.

Figure 4.1a-d. Four spectrograms showing examples of sounds recorded from North Atlantic right whale SAGs. a) A sequence of right whale Scream calls and Gunshots recorded from a North Atlantic right whale surface active group in August 2000. b) One call from the sequence shown in 1a) c) Another Scream call from the sequence shown in 1a) to illustrate the variability of Scream calls believed to be produced by one individual from a single surface active group and d) A Gunshot sound taken from the sequence of calls in 1a) to illustrate the presence of these sounds in surface active groups. 


\subsection{Methods}

\subsubsection{Location}

Playback trials were carried in the Bay of Fundy, Canada, east of Grand Manan Island (Fig. 4.2) from July through September in 1999, 2000 and 2001. The playbacks were conducted only when two or more right whales were visible from the playback platform.

\subsubsection{Playback Platforms}

Playbacks were carried out on four separate platforms. The two primary platforms were a $4.5 \mathrm{~m}$ rigid hull inflatable, the R/V Bonita (1999 and 2000), and the 22 $\mathrm{m} \mathrm{R} / \mathrm{V}$ Stellwagen (2001). A $7 \mathrm{~m}$ rigid hull inflatable boat (8/27/2000) and the $14 \mathrm{~m} \mathrm{~F} / \mathrm{V}$ Black-B (7/26/2000) were each used for one day of playback trials in 2000. All vessels were shut down and drifting during the playback experiment.

\subsubsection{Stimuli}

Three classes of stimuli were used: 1) calls from North Atlantic right whale surface active groups (NARW-SAGs) recorded in the Bay of Fundy and Brown's Bank by the New England Aquarium from the mid 1980's to early 1990's with all broadband Gunshot sounds removed (NARW, Fig. 4.1a-c), 2) Screams from South Atlantic right whale surface active groups (SARW-SAGs) recorded in Argentina in the late 1970's by Christopher W. Clark, with all broadband Gunshot sounds removed, and 3) broadband Gunshot sounds recorded from a lone North Atlantic right whale male in the Bay of Fundy in 2000 (NAGS, Fig. 4.1d).

Few high quality recordings of SAGs were available for North Atlantic right whales at the start of this study, therefore playback stimuli recorded from South Atlantic right whales were used to increase the number of high quality stimulus tapes available for the experiment. The NARW-SAG Screams were similar to the SARW-SAG Screams in 
terms of types of calls, frequency range, and time interval between calls. However, all NARW-SAG recordings used were made on the feeding grounds in summer, whereas all SARW-SAG recordings were made on the calving/nursery ground in Patagonia during the austral winter, covering different extremes of the annual migratory path in these two right whale populations. During the preliminary trials in the first year, 1999, a single 15 minute stimulus of NARW-SAG Screams (NARW2) was used. In 2000 six 20 minute stimuli ( 3 NARW, 3 SARW) were used. In 2001 seven 20 minute stimuli were used, including the six from 2000 with the addition of the NAGS stimuli.

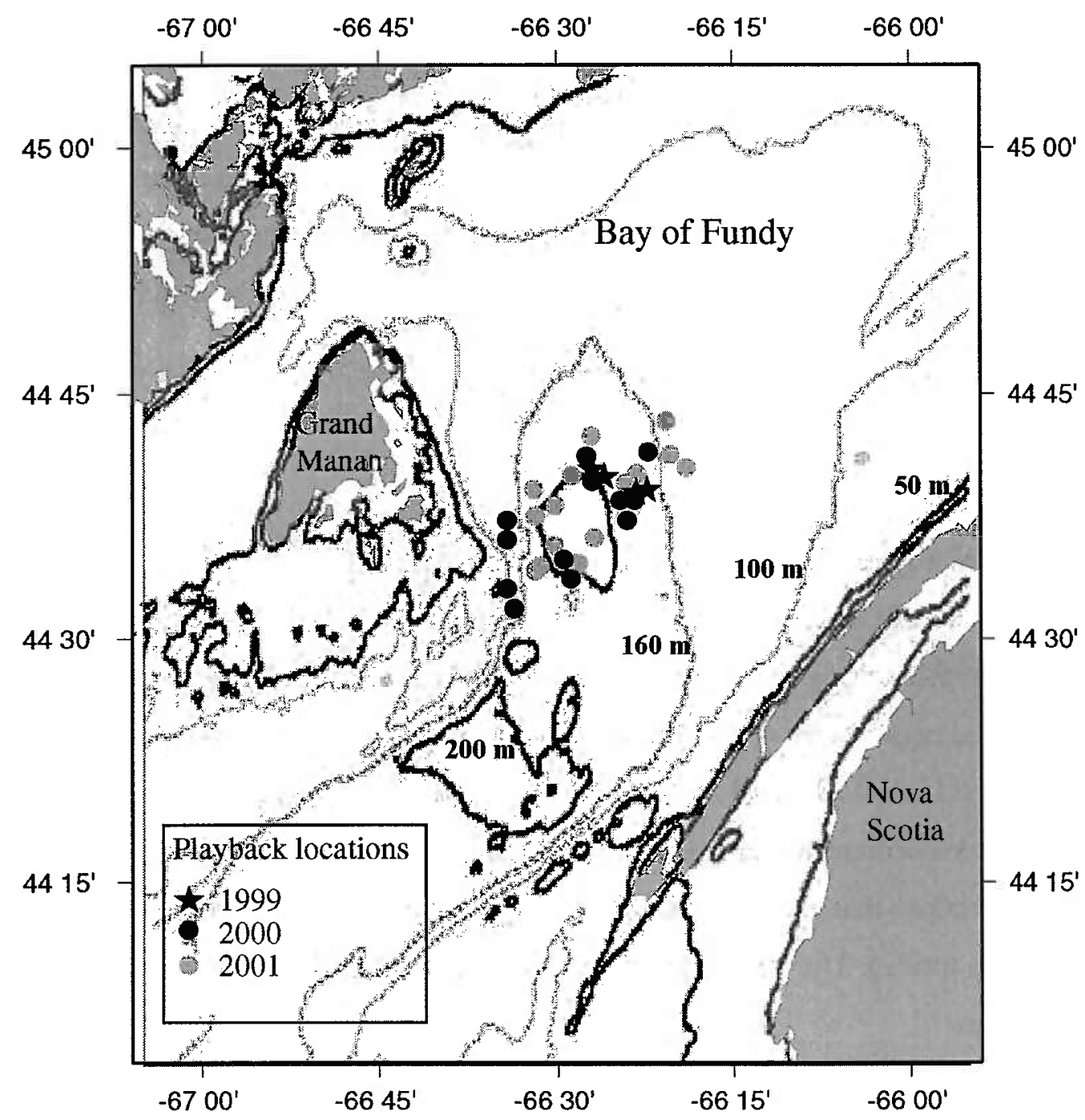

Figure 4.2. Bay of Fundy locations of playbacks between 1999 and 2001. 


\subsubsection{Equipment}

The frequency bandwidth characteristics of the equipment used for the sound playback were selected to match the natural stimuli as closely as possible. Two TASCAM DA-P1 DAT recorders (nominal frequency response $20 \mathrm{~Hz}-20 \mathrm{kHz}$ ) were used both to play and monitor the playback stimuli. A single Hi-Tech HTI-94-SSQ hydrophone (nominal frequency response $2 \mathrm{~Hz}-20 \mathrm{kHz}$ ) was used to monitor the playback stimuli while they were being broadcast and to record any sounds produced by whales near the playback vessel. Two amplifiers were used. In 1999 and 2000 a U.S. Acoustics $2 \times 300 \mathrm{~W}$ car stereo amplifier was used. In 2001, a Rockford-Fosgate Punch 800 amplifier was used. Both amplifiers were powered by a $12 \mathrm{~V}$ marine battery and provided the same output voltage $(\sim 100 \mathrm{~V}$ rms for the Screams) to the transducer. The stimuli were projected with a U.S. Navy Sound Reference Laboratory J-11 transducer (Nominal frequency response $20 \mathrm{~Hz}-20 \mathrm{kHz}$, flat frequency response $50 \mathrm{~Hz}-4 \mathrm{kHz}$ ).

\subsubsection{Projector source level}

The transducer had a maximum broadband source level of $163 \mathrm{~dB}$ re $1 \mu \mathrm{Pa}$ at $1 \mathrm{~m}$ for all stimuli types (bandwidth was approximately $30 \mathrm{~Hz}-20 \mathrm{kHz}$ ). Calculated maximum source level was approximately $160 \mathrm{~dB}$ re $1 \mu \mathrm{Pa}$ at $1 \mathrm{~m}$ for each stimulus. An estimate of the range at which transmission loss reduced the signal to equal ambient background noise levels at $250 \mathrm{~Hz}$ was $10 \mathrm{~km}$ from the source (Assumes spherical spreading (Urick, 1983) and ambient noise level of $80 \mathrm{~dB}$ re $1 \mu \mathrm{Pa} \wedge 2 / \mathrm{Hz}$, following short-term recordings made in the Bay of Fundy in 1999 (F. Desharnais, personal communication) that are in agreement with Wenz (Wenz, 1962) for areas with normal to heavy ship traffic). The transducer was deployed at a depth of $10 \mathrm{~m}$. Average water depth was $200 \mathrm{~m}$. 


\subsubsection{Playback protocol}

Each playback trial was divided into three observation periods; pre-, during and post-playback. Throughout each of these periods visual observations were made from the playback vessel to determine the right whale distribution and general behavior in the area (e.g. SAGs, traveling single whales, mother-calf pairs). The maximum range of the visible horizon varied from $4.5 \mathrm{~km}$ (height of eye $1.5 \mathrm{~m}$ ) to $10.5 \mathrm{~nm}$ (height of eye $7.6 \mathrm{~m}$ ) on different platforms. Approaching whales could often be seen at 4-5 km because they often swam rapidly at the surface, lifting their flukes frequently for shallow dives. Acoustic recordings were made with a single hydrophone during each of the three observation periods to monitor acoustic activity of whales around the playback vessel and to monitor the playback stimuli. During the preliminary 1999 trials, the three observation periods were 15 minutes in length. In 2000 and 2001 each period was increased to 20 minutes to allow more time for distant whales to reach the playback vessel. Visual observers measured the bearing, heading and range (estimated by observers trained with a laser range finder) to all right whales seen at the surface before the playback to determine right whale distribution in the area. The same data were collected during the playback and post playback periods. There were three levels of response to the playbacks. A no response score " 0 ", was given for a trial where all observed whales either did not change heading or were further from the playback vessel at the end of the playback than at the start. A moderate, level-one, response score " 1 " was given for trials where at least one whale approached to within $500 \mathrm{~m}$ of the playback vessel during the playback or post playback periods. The range of $500 \mathrm{~m}$ was selected to be conservative in determining an approach because visibility was limited from low observation platform in 1999 and 2000. A strong, level-two, response score " 2 " was given for trials where at least one whale swam rapidly to within one right whale body length of the playback vessel during the playback or post playback periods.

On any given day in 2000 and 2001 playback stimuli were presented in a random order pre-determined at the beginning of the field season. Observations were conducted 
from the playback vessel so it was not possible to have observers blind to the playback condition and observers could hear the transmission of the playback sounds through the hull in 1999 and 2000. Only SAG stimuli were presented in 1999 and 2000. However, although observers knew when a playback was taking place, they did not know which type of stimulus was being played, and therefore their observations should not be biased.

Photographs to identify individuals were taken of all animals that approached the playback vessel closely enough to obtain clear images of distinguishing features $(n=52)$. In some cases no whales approached the vessel closely enough for clear photographs. This was particularly a problem in 1999 and 2000 when the playback platform was usually a small inflatable that was low to the water. In 1999, 2000, and 2001 photographs of non-responding individuals were collected opportunistically from other research vessels in the area during playback trials $(n=69)$. These photo-identifications of non-responding individuals, while not systematically collected, allow us to compare which animals responded or did not respond during each playback trial.

The playback location was selected by finding a place with at least two right whales visible from the playback vessel. This ensured that at least two individuals were within range to hear the playback. This experiment differed from more traditional playback studies because there was no single focal subject followed throughout the playback trial. Due to the difficulties of working with baleen whales at sea, the age, sex class and total number of potential responders within hearing range of the playback were unknown. The paradigm of these playback experiments was such that a yes/no ( 0 vs. 1 or 2) criteria for each trial (not each individual) was the basis for testing the significance of differences in response to different stimuli. This yes/no criterion was based on observations from similar playback experiments to South Atlantic right whales (Clark \& Clark, 1980) and humpback whales (Tyack, 1983). Differences in response to different stimuli were tested using Fischer's exact probability test (Hinkle \& Wiersma, 1998). The prediction was that primarily adult males would approach the playbacks of SAG Screams. The limitation of this design is that lack of obvious approach may be a function of lack of adequate exposure to the stimuli, due to noise or distance from the source, 
rather than an active choice on the part of a whale. The overwhelming strength of response from individual whales, coupled with opportunistic identification of whales within presumed hearing range of the playback, indicate that some juveniles and adults of both sexes had the opportunity to respond to at least one trial of each stimuli.

\subsection{RESULTS}

\subsubsection{Response to the playbacks}

Thirty-six playback trials were conducted (Table 4.1). The playback of Screams from surface active groups (SAGs) elicited swimming approaches that were similar to those of whales approaching actual SAGs. Fig. 4.3 shows an example of observations collected during a NARW-SAG playback trial. In this playback, four whales were visible before the playback started at 13:59. Two whales turned and began to swim toward the playback vessel from the west within three minutes of the start of the playback. Two whales that had not been seen before were sighted heading toward the playback during the trail. One whale swam past the vessel and continued to head away after the playback started. The four whales that approached the playback vessel joined together and formed a SAG off the vessel twenty minutes after the end of the playback. Only gunshot sounds were recorded from the group. 


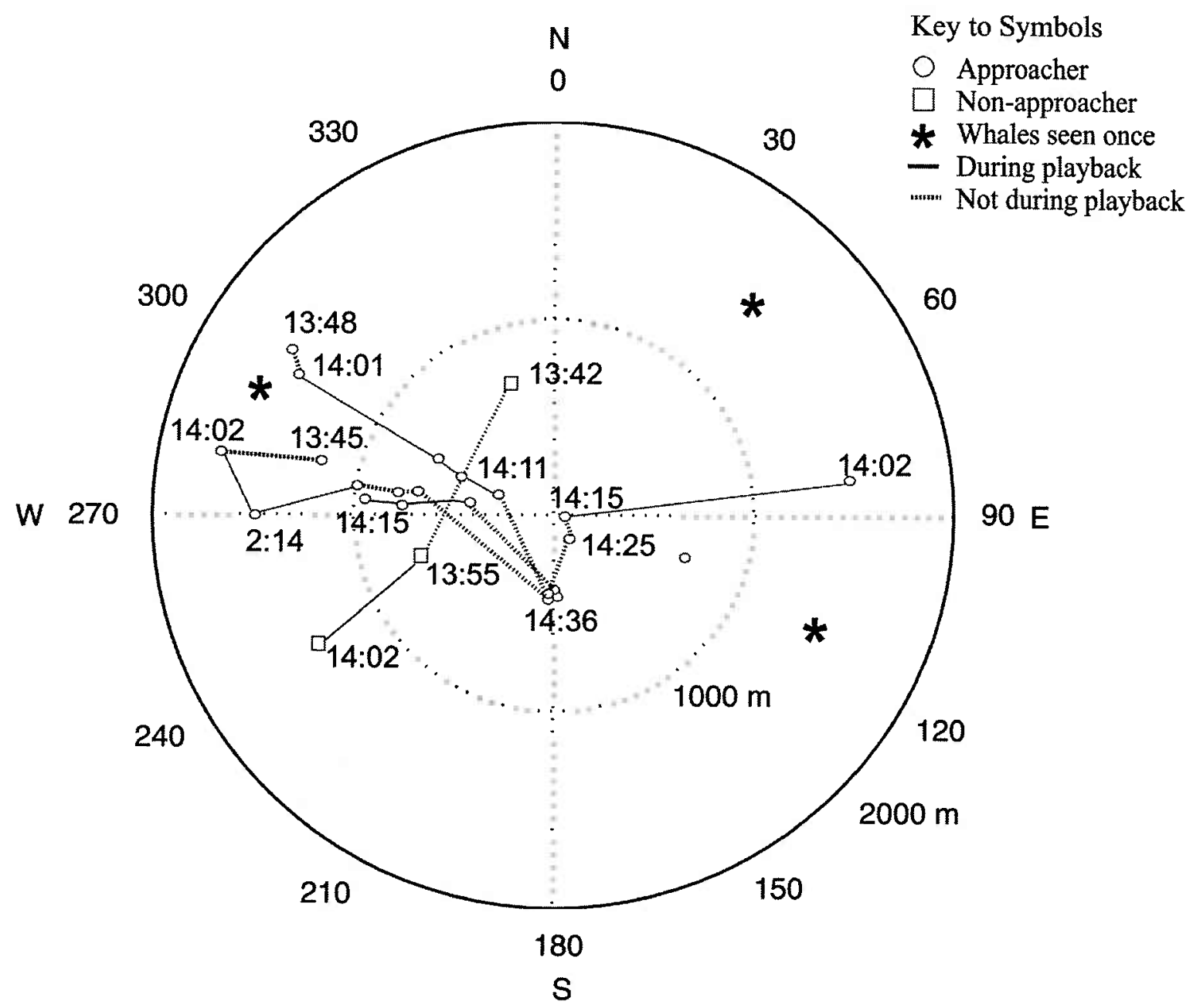

Figure 4.3. Sighting positions and tracks of individual whales $(n=8)$ seen within $2000 \mathrm{~m}$ of the playback vessel (center of the plot) during the playback trial on 31 August 2000. The trial started at 13:39 h. The playback started at 13:59 h and lasted until 14:19h. Dashed or solid lines are used to connect sightings for which there was a high level of confidence that the sightings were of the same individual. Sightings made during pre- and post-playback periods are shown by dashed lines. Sightings made during the playback are shown by solid lines. Four whales approached the playback vessel during this trial but only two were positively identified. All four joined together and formed a short lived SAG off the playback vessel at $14: 36 \mathrm{~h}$. 
Table 4.1. The date, stimulus type, level of response, number of whales seen from the playback vessel during a trial, the number of whales that approached the playback vessel during a trial, the age and sex of whales that approached the playback vessel and age and sex of identified whales that did not approach the playback vessel are given in the table. For the identity columns, information about sex and age is given. $\mathrm{M}=\mathrm{Male}, \mathrm{F}=\mathrm{Female}, \mathrm{U}=\mathrm{Unknown}$ sex, $\mathrm{A}=\mathrm{Adult}(>8$ years), $\mathrm{J}=$ Juvenile $(<9$ years), $\mathrm{C}=\mathrm{Calf}(<1$ years $), \mathrm{U}=$ Unknown age.

\begin{tabular}{|c|c|c|c|c|c|c|}
\hline Date & Stimulus & $\begin{array}{l}\text { Response } \\
\text { level (0-2) }\end{array}$ & $\begin{array}{c}\text { Number of } \\
\text { whales seen } \\
\text { during } \\
\text { playback }\end{array}$ & $\begin{array}{c}\text { Number of } \\
\text { whales } \\
\text { approaching } \\
\text { playback vessel }\end{array}$ & $\begin{array}{c}\text { Sex/Age of } \\
\text { Identified } \\
\text { Approaching } \\
\text { whales } \\
\end{array}$ & $\begin{array}{c}\text { Sex/Age of Identified } \\
\text { non-approaching whales }\end{array}$ \\
\hline $7 / 31 / 2001$ & NAGS & 0 & 9 & 0 & & 1FA, $1 \mathrm{FJ}, 1 \mathrm{UC}$ \\
\hline $8 / 9 / 2001$ & NAGS & 0 & 5 & 0 & & 1UC \\
\hline $8 / 11 / 2001$ & NAGS & 0 & 11 & 0 & & $\begin{array}{c}4 \mathrm{FA}, 1 \mathrm{FJ}, 1 \mathrm{MA}, 1 \mathrm{MJ}, \\
1 \mathrm{UC}\end{array}$ \\
\hline $8 / 14 / 2001$ & NAGS & 0 & 13 & 0 & & 2FA,2UC \\
\hline $8 / 19 / 2001$ & NAGS & 0 & 17 & 0 & & 5FA, 2UU, 1UC \\
\hline $7 / 15 / 2000$ & NARW1 & 1 & 3 & 2 & $1 \mathrm{MA}, 1 \mathrm{MJ}$ & \\
\hline $8 / 29 / 2000$ & NARW1 & 2 & 10 & 1 & $1 \mathrm{MA}$ & \\
\hline $8 / 31 / 2000$ & NARW1 & 2 & 6 & 2 & $2 \mathrm{MA}$ & \\
\hline $7 / 21 / 2001$ & NARW1 & 0 & 8 & 0 & & 2FA, 3UC \\
\hline $8 / 1 / 2001$ & NARW1 & 1 & 12 & 1 & & $1 \mathrm{FA}, 1 \mathrm{FJ}, 2 \mathrm{UC}$ \\
\hline $7 / 21 / 1999$ & NARW2 & 1 & 4 & 2 & & \\
\hline $8 / 20 / 1999$ & NARW2 & 2 & 4 & 3 & 2MA, 1UA & \\
\hline $8 / 20 / 1999$ & NARW2 & 2 & 1 & 1 & $1 \mathrm{UA}$ & \\
\hline $8 / 20 / 1999$ & NARW2 & 2 & 2 & 2 & 1MA, 1MU & \\
\hline $8 / 20 / 1999$ & NARW2 & 2 & 3 & 3 & $3 \mathrm{MA}$ & \\
\hline $7 / 15 / 2000$ & NARW2 & 2 & 3 & 1 & & \\
\hline $8 / 27 / 2000$ & NARW2 & 2 & 3 & 1 & $1 \mathrm{MA}$ & \\
\hline $8 / 11 / 2001$ & NARW2 & 2 & 16 & 5 & $4 \mathrm{MA}$ & 1FA, 2MA, 1MJ, 1UC \\
\hline $7 / 26 / 2000$ & NARW3 & 1 & 10 & 2 & 1MA, 1UA & 2FA, 1MU, 1UU \\
\hline $8 / 29 / 2000$ & NARW3 & 1 & 15 & 7 & $5 \mathrm{MA}, 1 \mathrm{MJ}$ & \\
\hline $8 / 31 / 2000$ & NARW3 & 1 & 5 & 1 & & \\
\hline $7 / 27 / 2001$ & NARW3 & 0 & 8 & 0 & & 1FA, 2MA, 2UC \\
\hline $8 / 4 / 2001$ & NARW3 & 2 & 15 & 7 & $4 \mathrm{MA}$ & 3FA, 1UC \\
\hline $7 / 26 / 2000$ & SARW1 & 1 & 6 & 5 & $\begin{array}{c}\text { 1FA, 1FJ, } \\
\text { 1MA,1MJ, 1MU }\end{array}$ & \\
\hline $7 / 27 / 2001$ & SARW 1 & 1 & 13 & 3 & $1 \mathrm{MA}, 1 \mathrm{MU}$ & \\
\hline $8 / 14 / 2001$ & SARW 1 & 0 & 15 & 0 & & $\begin{array}{c}\text { 1FA, 1FJ, 1MA, 3MJ, } \\
\text { 1UC }\end{array}$ \\
\hline $8 / 24 / 2001$ & SARW1 & 2 & 7 & 1 & $1 \mathrm{MA}$ & $1 \mathrm{UC}$ \\
\hline $8 / 29 / 2000$ & SARW2 & 1 & 1 & 1 & $1 \mathrm{MA}$ & \\
\hline $7 / 28 / 2001$ & SARW2 & 1 & 12 & 3 & $2 \mathrm{UC}$ & 1FA, 1MA, 1UC \\
\hline $8 / 11 / 2001$ & SARW2 & 2 & 10 & 2 & 1FA & \\
\hline $8 / 19 / 2001$ & SARW2 & 2 & 15 & 5 & $2 \mathrm{FA}, 1 \mathrm{MA}, 2 \mathrm{UC}$ & $2 U U$ \\
\hline $7 / 26 / 2000$ & SARW3 & 1 & 6 & 1 & $1 \mathrm{UA}$ & \\
\hline $8 / 31 / 2000$ & SARW3 & 2 & 6 & 1 & $1 \mathrm{FA}$ & \\
\hline $7 / 29 / 2001$ & SARW3 & 0 & 6 & 0 & & $1 F A$ \\
\hline $8 / 11 / 2001$ & SARW3 & 1 & 14 & 3 & $1 \mathrm{UA}$ & $2 \mathrm{FA}, 1 \mathrm{FJ}, 1 \mathrm{MA}$ \\
\hline $8 / 25 / 2001$ & SARW3 & 1 & 11 & 2 & 1FA, 1UC & \\
\hline
\end{tabular}


All six SAG playback stimuli from the North Atlantic (NARW-SAG 1-3) and the South Atlantic (SARW-SAG 1-3) elicited level-two responses on at least one occasion, during which whales charged the playback platform (high speed surface swimming, directly at the playback vessel) and swam under the boat. Overall, at least one whale approached the playback vessel in 27 of 31 playback trials of SAG Screams (Fig.4.4a). The NARWSAG stimuli elicited approach responses on 16/18 trials and the SARW-SAG stimuli elicited approach responses on 11/13 trials. There was no significant difference in the proportion of trials with approaches for the NARW and SARW SAG stimuli $(P=0.57$, ns); Fisher exact probability test, (Hinkle \& Wiersma, 1998) (Fig. 4.4a). No individuals approached any of the five playbacks of the Gunshot sounds (NAGS) (Table 4.1) indicating a significant difference in response to SAG and Gunshot sound playbacks $(P<$ 0.0003 ) (Fig. 4.4b).

A)

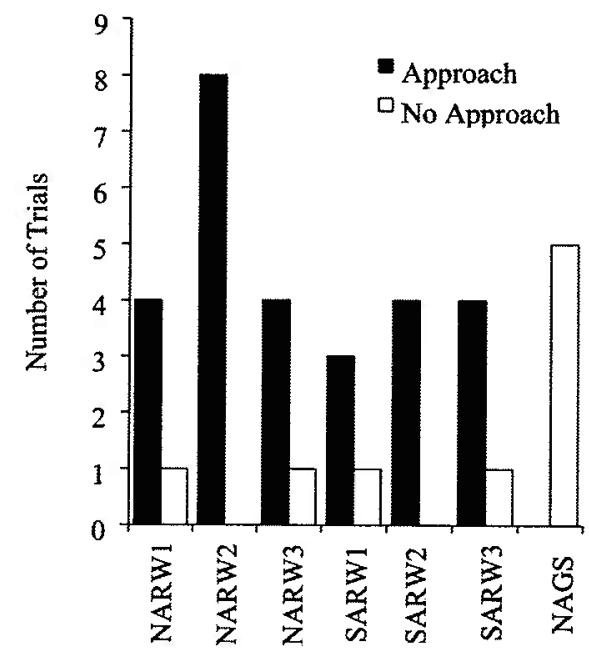

B)

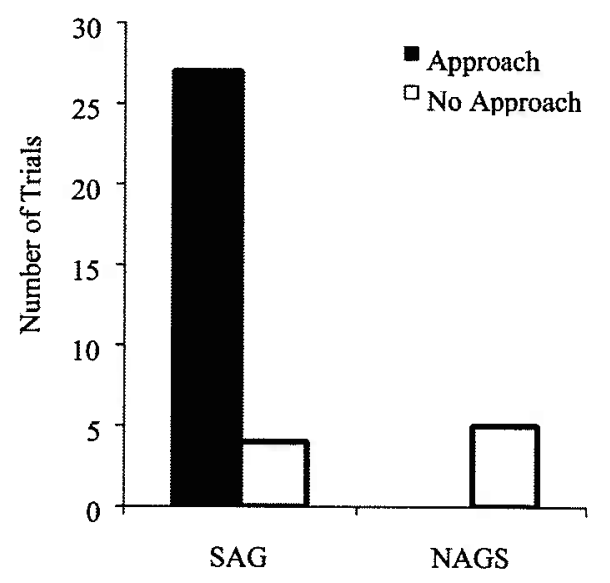

Figure 4.4. A) Approach response vs. no approach response observed in each trial for each of the seven stimuli presented for playback. There was no significant difference between any of the SAG playback stimuli. B) Approach response vs. no approach response to SAG $(n=31)$ and Gunshot $(n=5)$ playbacks (NAGS). The difference in approach vs. no approach between SAG and Gunshot stimuli was significant $(P$ $=0.0003$ ). 
An average of $2.1 \pm 1.9 \mathrm{SD}$, (range 0-6) individuals approached the playback vessel while as many as 17 right whales were seen before a playback commenced (Table 4.1). Many whales exposed to the playbacks did not show any clear evidence of response. For the NARW-SAG stimuli, most of these individuals were calves, adult females or juvenile males (Table 4.2). For every playback stimulus, there were individuals that did not respond and these individuals were often closer to the playback vessel at the start of the playback than the whales that did approached the vessel. The identification of the non-approaching whales (Table 4.2) indicate that whales of both sexes were exposed and did not respond to both NARW-SAG and SARW-SAG stimuli.

Table 4.2. Summary of the age and sex of whales approaching and not approaching the playback vessel during A) NARW-SAG B) SARW-SAG playbacks. Adults are > 8years.

A

\begin{tabular}{|c|c|c|c|c|c|c|}
\hline & \multicolumn{3}{|c|}{ Whales Approaching NARW-SAG } & \multicolumn{3}{|c|}{ Whales Not Approaching NARW-SAG } \\
\hline & Male & Female & Unknown Sex & Male & Female & Unknown Sex \\
\hline Adult & 25 & 0 & 3 & 4 & 10 & 0 \\
\hline Juvenile & 2 & 0 & 0 & 1 & 1 & 0 \\
\hline Calf & 0 & 0 & 0 & 0 & 0 & 9 \\
\hline Unknown & 1 & 0 & 0 & 1 & 0 & 1 \\
\hline
\end{tabular}

B

\begin{tabular}{|c|c|c|c|c|c|c|}
\hline & \multicolumn{3}{|c|}{ "Whales Approaching SARW-SAG } & \multicolumn{3}{|c|}{ Whales Not Approaching SARW-SAG } \\
\hline & Male & Female & Unknown Sex & Male & Female & Unknown Sex \\
\hline Adult & 5 & 6 & 2 & 3 & 5 & 0 \\
\hline Juvenile & 1 & 1 & 0 & 3 & 2 & 0 \\
\hline Calf & 0 & 0 & 5 & 0 & 0 & 3 \\
\hline Unknown & 2 & 0 & 0 & 0 & 0 & 2 \\
\hline
\end{tabular}

In the typical level-one response, whales approached the playback slowly, often taking most of the 20 minute playback session to come within $500 \mathrm{~m}$ of the playback vessel. Other whales approached the vessel subsurface after diving at least $1 \mathrm{~km}$ away. Similar approaches have been seen to actual SAGs with whales positioned near the SAG 
for a period of time before joining the group. For the level 2 response, whales would commonly approach the boat by "racing diving", a term used to describe whales traveling at relatively high speeds near the surface, making repeated shallow dives with flukes raised at an angle of about $45^{\circ}$ after each blow. A "racing diving" whale was easy to distinguish from other whales because of its high rate of travel, frequently raised flukes, and directed straight line path of travel towards the playback vessel. Several approaching whales were observed swimming directly under the playback vessel $(n=18)$ during the trial. On two occasions whales bumped into the transducer and released a cloud of bubbles next to the boat. Acoustic recordings made from the playback vessel recorded Upcalls on several occasions during and after the playback trials of SAG sounds. Gunshot sounds were heard on one occasion when the approaching whales formed a SAG near the playback vessel.

\subsubsection{Differential response between sexes}

Individual identification made it possible to determine the age and sex for many of the whales involved in each playback trial. There appear to be differences in the types of individuals that showed response for all three classes of stimuli (NARW-SAG, SARW-SAG, NAGS). Only the NARW-SAG Screams elicited differential response between the sexes. Most whales approaching NARW-SAG sounds were adult males and no known females were observed to approach these stimuli (Table 4.2a). For the 16 cases when the sex of a "racing diving" approaching whale could be determined for the NARW-SAG playbacks, it was always male. Ten adult females were identified during NARW-SAG playbacks but none approached the playback vessel (Table 4.2a). All age and sex classes were observed to approach the SARW-SAG sounds (Table 4.2b). Most notably, six adult females approached the SARW-SAG sounds but none approached the NARW-SAG sounds. Females were observed "racing diving" during SARW-SAG playbacks on three occasions. The observed response in the field was comparable for both NARW-SAG and SARW-SAG playbacks. It was only later when the identity of individuals was determined by photo analysis that the differences emerged. No whales 
approached the playbacks of Gunshot sounds even though whales of all age and sex classes were documented in the area during these playbacks (Table 4.1).

\subsection{Discussion}

Playbacks of Screams recorded from surface active groups (SAGs) were predicted to attract North Atlantic right whales based on observations of right whale behavior in SAGs in the Bay of Fundy. If SAGs with a single focal female function for reproduction then one would expect males to be the primary responders to SAG playbacks. All identified individuals of known sex seen approaching actual SAGs have been adult males (Kraus \& Hatch, 2001).

The responses to all three NARW-SAG stimuli support the initial prediction that NARW-SAG Scream calls are what attract adult male North Atlantic right whales to SAGs. The overwhelmingly strong response to these playbacks, often involving very rapid surface swimming and persistent searching (swimming back and forth over a small area around the playback vessel) within $500 \mathrm{~m}$ for the sound source for up to 20 minutes after the end of the playback by adult males $(n=2)$ (or adults of unknown sex $(n=1)$ ) support the idea that the Scream calls produced in SAGs do attract the males that join these groups. Whales that were identified as not approaching the NARW-SAG stimuli were predominantly female (Table 4.2a). This result also corresponds with observations in the field, where fewer juvenile males or adult females are seen in the typical SAG containing a mature focal female. None of the whales that approached the playbacks produced Scream calls. The only sounds recorded near the playback vessel on the single monitoring hydrophone after (or during) playbacks were Gunshot sounds or Upcalls, which are considered to be a form of contact call in the South Atlantic right whale repertoire (Clark, 1983). These Upcalls have been recorded from males as they approach SAGs (Chapter 3).

The results from the Gunshot playbacks indicate that right whales do not approach every type of right whale sound stimulus projected to them. Only a single Gunshot 
stimulus was used, so whether this response generalizes to other exemplars from this class of signal remains uncertain (Searcy, 1989). Although Gunshot sounds are typically recorded from SAGs, it appears that the Scream calls recorded from NARW-SAGs are alone sufficient to attract males. The Gunshot sounds often overlap with other Gunshot sounds and Scream calls, suggesting that more than one animal in the SAGs produce these sounds. It is therefore most likely that males in SAGs produce Gunshot sounds as more Gunshots seem to be heard with an increasing number of males present in SAGs (Chapter 3) and all observed lone animals producing these Gunshot sounds in the Bay of Fundy $(n=3)$ have been male. A male hearing Gunshot sounds may only know that other males are interacting, with no guarantee that a female is involved. One NARWSAG playback resulted in four approaching whales coming together and forming a brief 10-15 minute SAG approximately $300 \mathrm{~m}$ off the playback vessel (Fig. 4.3). Only two of the animals approached the vessel closely enough to be positively identified, and both of these were adult males. The hydrophone recordings made during and after this playback only picked up very loud Gunshot sounds presumably produced by a whale or whales close to the vessel. No other whales were visible from the playback platform at that time and it is probable, given the sex of whales approaching other NARW stimuli that this SAG consisted only of males.

The SARW-SAG trials produced the most unexpected results from this experiment. All age and sex classes were seen to approach during these trials (Table 4.2b). The SARW playback stimuli were selected to increase the number of stimuli tapes. They were high quality recordings with very similar call types and call rates to those made from NARW-SAGs. The predictions for these playbacks were the same as those for NARW-SAG playbacks. However, these results document a clear difference in response by North Atlantic right whales to North and South Atlantic right whale SAG sounds. There are several possible reasons for the observed differences in response. The first possibility is that the Scream calls of these two species differ enough so as not to be recognized by North Atlantic right whales as right whale calls. Secondly, if right whales recognize the Scream calls of specific individuals, then the Scream calls from the SARW- 
SAGs were from individual whales that were completely unknown to the North Atlantic right whale population. In both cases, the novelty of these stimuli might attract attention from any member of the North Atlantic right whale population. A third potential explanation for the difference is that these recordings were made from the calving grounds of a SARW population which has a different demographic structure and behavioral context than that seen on the feeding grounds in the North Atlantic. SAGs have been observed in all known habitats for the North Atlantic right whale and in eight months of the year (Kraus \& Hatch, 2001) but it is unclear whether the SAGs in different habitats serve the same social or reproductive function, and sounds produced by SAGs outside of the Gulf of Maine during the summer months have not been recorded for this species. SAGs on the calving ground might serve a different function and differences in frequency, call modulation or some other factor might signal this difference to right whales in both the North and South Atlantic.

Lower levels of response ( 0 and 1$)$ were observed during the month of July than in August or September. Playbacks in July elicited fewer approaches than those carried out in August or September (of the four SAG playbacks with no clear response, three were conducted in July). Males appeared to be making increased effort to get to SAGs as the summer season in the Bay of Fundy progressed. More individuals were seen approaching both playbacks and actual SAGs by "racing diving" in late August than in July (Table 4.1), and SAG size has been shown to increase throughout the summer months (Kraus \& Hatch, 2001). This suggests that there may be increasing motivation on the part of males to join SAGs as the summer progresses. The observation of more allmale SAGs in the early fall is consistent with this idea (Chapter 2).

There are limitations to any playback experiments to North Atlantic right whales. The detailed demographic information is primarily available because this is a highly endangered species, with fewer than 300 individuals left in the entire population. While this detailed demographic information increases insight into the response of individuals, it comes with the cost of having few potential test subjects in a small population. The large proportion of the North Atlantic right whale population that congregates on the 
summer feeding grounds where these playbacks were conducted compounds this problem. The source level and frequency range of the playback suggest that it may be audible above ambient noise for approximately $10 \mathrm{~km}$ from the source. There are times in the Bay of Fundy when the whales are all feeding in a very small geographic area; as a result, at these times, up to a quarter of the entire population could potentially be exposed to each playback trial. Therefore, after the first trial of each stimulus, it is not possible to ensure lack of previous exposure to the playback. The North Atlantic playback recordings were made on the same feeding grounds, meaning that a large proportion of the population may have been exposed to the actual sounds during the original SAG. While the playbacks occurred 8-15 years after the original event, the signals may still be familiar to whales in the population if the signals are individually distinctive. Some individual males (e.g. Catalog ID \#1304 and 1901) approached the same stimuli (NARW2 and NARW3, respectively) on more than one occasion, which may be a random consequence of the whale being in the right place at the right time, or it may indicate higher motivation in these individuals for the Scream calls recorded from a particular SAG. The results from these playback trials cannot be evaluated without taking these factors into account. Efforts were made throughout this experiment to 1) limit the number of playbacks made on a given day, 2) conduct playbacks throughout the season (because the population in the bay changes during the season) and, 3) use several different examples of stimuli for each class of signal. Aside from the first playback trials of the SARW-SAG stimuli, there is the possibility that all potential test subjects may have had previous exposure to the stimulus being presented. However, if SAG sounds signal mating opportunities for males, then one should expect strong selection against habituation.

This playback experiment demonstrates that Scream calls produced by right whales in SAGs do have behavioral significance to individuals. Adult males show the strongest responses to playbacks of NARW-SAG Scream calls, which was predicted by observations of behavior in real SAGs. The approach to SAG sounds by adult males supports the assumption that females produce the Scream calls in SAGs. The difference 
in the age and sex composition of the whales that approached the SARW-SAG Scream calls demonstrates that behaviorally significant variation exists in response to this broad class of sounds. Finally, the responses of whales to these playbacks indicate that right whales have the ability to accurately locate a sound source and remember where the sound source was for at least 20 minutes after the broadcast of sound stops. These results suggest that further studies of right whales' perceptual and cognitive abilities may be warranted. Playback trials, coupled with newly developed tagging methods (Johnson \& Tyack, 2003), can be used to look at fine scale motor response and record the received level of a signal to address hearing and detection abilities of individual free-ranging whales (Appendix 3).

\subsection{LITERATURE CITED}

Brown, M. W., Kraus, S. D., Gaskin, D. E. \& White, B. N. 1994. Sexual composition and analysis of reproductive females in the North Atlantic right whale, Eubalaena glacialis, population. Marine Mammal Science, 10, 252-65.

Brownell, R. L. 1986. Potential for Sperm Competition in Baleen Whales. Rep. Int. Whal. Commn. (Special Issue 8), 97-112.

Brownell, R. L. J. \& Ralls, K. 1986. Potential for Sperm Competition in Baleen Whales. Rep. Int. Whal. Commn. (Special Issue 8), 97-112.

Clark, C. W. 1983. Acoustic communication and behavior of the southern right whale. In: Behavior and communication in whales. American Association for the Advancement of Science Selected Symposium 76 (Ed. by Payne, R. S.), pp. 163198. Boulder, CO.: Westview Press.

Clark, C. W. \& Clark, J. M. 1980. Sound Playback Experiments with Southern Right Whales (Eubalaena australis). Science, 207, 663-665.

Cummings, W. C. \& Thompson, P. O. 1971. Gray whales, Eschrichtius robustus, avoid the underwater sounds of killer whales, Orcinus orca. Fishery Bulletin, 69, 525530.

Frankel, A. S., Joseph R. Mobley, Jr. , Louis M. Herman. 1995. Estimation of auditory response thresholds in humpback whales using biologically meaningful sounds. 
In: Sensory Systems of Aquatic Mammals (Ed. by R.A. Kastelein, J. A. T. a. P. E. M.), pp. 55-70. Woerden, The Netherlands: De Spil Publishers.

Harcourt, A. H., Harvey, P. H., Larson, S. G. \& Short, R. V. 1981. Testis weight, body weight and breeding systems in primates. Nature, 293, 55-57.

Hinkle, D. E. \& Wiersma, W. 1998. Applied Statistics for the behavioral sciences. New York: Houghton Mifflin Company.

IWC. 2001. Report of the workshop on status and trends of western North Atlantic right whales. The Journal of Cetacean Research and Management (special issue) 2, 6187.

Johnson, M. P. \& Tyack, P. L. 2003. A digital acoustic recording tag for measuring the response of wild marine mammals to sound. IEEE Journal of Oceanic Engineering, 28, 3-12.

Kenagy, G. J. \& Trombulak, S. C. 1986. Size and function of mammalian testes in relation to body size. Journal of Mammalogy, 67, 1-22.

Knowlton, A. R., Kraus, S. D. \& Kenny, R. D. 1994. Reproduction in North Atlantic right whales (Eubalaena glacialis). Canadian Journal of Zoology, 72, 297-305.

Kraus, S. D., Hamilton, P. K., Kenney, R. D., Knowlton, A. R. \& Slay, C. K. 2001. Reproduction parameters of the North Atlantic right whale. The Journal of Cetacean Research and Management. (Special issue) 2, 231-236.

Kraus, S. D. \& Hatch, J. J. 2001. Mating strategies in the North Atlantic right whale (Eubalaena glacialis). The Journal of Cetacean Research and Management (Special issue) 2, 237-244.

Kraus, S. D., Moore, K. E., Price, C. A., Crone, M. J., Watkins, W. A., Winn, H. E. \& Prescott, J. H. 1986. The use of photographs to identify individual North Atlantic right whales (Eubalaena glacialis). Report of the International Whaling Commission (Special Issue) 10, 145-151.

Møller, A. P. 1989. Ejaculate quality, testes size and sperm production in mammals. Functional ecology, 3, 91-96.

Norris, K. S., Villa-Ramirez, B., Nichols, G., Wüsig, B. \& Miller, K. 1983. Lagoon entrance and other aggregations of gray whales (Eschrichtius robustus). In: Communication and Behavior of whales (Ed. by Payne, R.), pp. 259-293. Boulder, CO: Westview Press. 
Omura, H., Ohsumi, S., Nemoto, T., Nasu, K. \& Kasuya, T, 1969. Black right whales in the North Pacific. Scientific Report of the Whales Research Institute, Tokyo, 21, 178 .

Searcy, W. A. 1989. Pseudoreplication, external validity and the design of playback experiments. Animal Behaviour, 38, 715-719.

Silber, G. K. 1986. The relationship of social vocalizations to surface behavior and aggression in the Hawaiian humpback whale (Megaptera novaeangliae). Canadian Journal of Zoology, 64, 2075-2080.

Tyack, P. 1983. Differential response of humpback whales, Megaptera novaeangliae, to playback of song or social sounds. Behavioral Ecology and Sociobiology, 13, 4955.

Urick, R. J. 1983. Principles of underwater sound. Los Altos, CA: Peninsula Publishing.

Wenz, G. M. 1962. Acoustic ambient noise in the ocean: spectra and sources. Journal of the Acoustical Society of America, 34, 1936-1956.

Winn, H. E., Price, C. A. \& Sorensen, P. W. 1986. The distributional biology of the right whale (Eubalaena glacialis) in the western North Atlantic. Report of the International Whaling Commission. (Special issue), 10, 129-138.

Würsig, B. \& Clark, C. W. 1993. Behavior. In: The Bowhead Whale (Ed. by Cowles, C. J.), pp. 157-199. Lawrence, KS. 
Chapter 4: Playbacks 


\section{CHAPTER 5: HEARING IN THE NORTH ATLANTIC RIGHT WHALE: ANATOMICAL PREDICTIONS}

\subsection{INTRODUCTION}

The oceanic environment of baleen whales necessitates the use of sound for communication. In the clearest ocean water, visibility is limited to a few hundred meters at the surface. In the highly productive areas where baleen whales feed, visibility is often reduced to less than a meter. In contrast, transmission of sound in the oceanic environment is very efficient, allowing for the potential to communicate over hundreds of kilometers (Payne \& Webb, 1971). Given these environmental constraints, it is likely that baleen whales have adopted sound as their primary means of communication, making it vital to understand this aspect of their biology. Acoustic communication includes sound production, transmission, reception and categorization. Most research on communication focuses on the sounds produced by baleen whales because, while still difficult to study, it is much easier to investigate sound production than what baleen whales hear and how they react to sound.

The North Atlantic right whale is a large baleen whale. The extant population is primarily located off the east coast of North America in an area with high levels of ship traffic and high ambient noise levels. The range at which right whales can detect each others calls may be limited by increasing levels of noise. This chapter will describe the anatomy of right whale inner ears to estimate their hearing sensitivities and discuss whether there is significant overlap of the frequency range of their hearing and anthropogenic noise sources.

\subsubsection{Hearing studies on other animals - A brief history}

Interest in hearing and acoustics have a long history, traceable as far back as Greek philosophers (Yost, 2000). Most of the current knowledge about hearing is a result of studies that began in the mid-1800's. Much auditory research is focused on increasing our understanding of human hearing. Studies of hearing in animals are equally long- 
standing, with some researchers interested in answering the question, "To what do animals listen?".

Valid animal psychoacoustics data have been collected since the mid-1930's when animal conditioning techniques were used to determine the audiogram for the chimpanzee (Elder, 1934). Since that first animal audiogram, further audiograms have been collected for over 60 mammalian species (Fay, 1994). These studies have revealed wide variation in the frequency range of hearing for different mammalian species. The entire range of hearing ability spans a range from $10 \mathrm{~Hz}$ to $200 \mathrm{kHz}$, although no single species' hearing covers the entire range (Fay, 1994).

\subsubsection{Anatomical correlates with hearing sensitivity}

The frequency range of hearing and sensitivity of mammalian ears are determined primarily by the resonance and filter characteristics of peripheral auditory system components. There are three major parts to the typical mammalian peripheral auditory system: 1) an outer ear which captures sound, 2) a middle ear which mechanically filters and amplifies sounds, and 3) an inner ear which is responsible for the transduction of sound into neural impulses. Species-specific hearing abilities are determined by the specific structure of these three regions. Because different species have different ear structures, they perceive incoming signals with different facility. Models that estimate hearing for each species are based on calculations of admittance and resonance derived directly from mechanical properties of the ear that are in turn, based on middle and inner ear anatomies. These modeling techniques are well established and provide reliable estimates of normal hearing ranges for most terrestrial mammals (Fay, 1992).

Comparative anatomical studies have identified structural correlates to frequency range and relative sensitivity of hearing in different mammalian species (Echteler et al., 1994). Differences have been observed in cochlear structure in species with different frequency ranges of hearing. Mammalian inner ears have been divided into two major categories, generalist and specialist. Generalist ears tend to have basilar membranes that have a common stiffness gradient allowing for frequency distributions to be calculated 
based on membrane length where as specialist cochleae have regions of morphological specializations for processing sound in particular frequency regions (Ketten, 2000).

Functional studies of the inner ear focus on resonance characteristics of the basilar membrane. G. von Békésy (1960) experimentally obtained position-frequency maps and basilar membrane elasticity measurements for 6 mammals and 1 bird. These data were the foundation for Greenwood's formulae for predicting frequency maxima, minima, and distribution along the length of the basilar membrane for any land mammal (Greenwood, 1961; Greenwood, 1962; Greenwood, 1990) (Formula 1). In this formula, A = a scaling factor to obtain frequency in $\mathrm{Hz}, \mathrm{a}=$ slope of the straight portion of the frequency position function, $\mathrm{x}=$ distance of a point along the basilar membrane and $\mathrm{k}=$ an integration constant. For example, the values for the human hearing curve would be $\mathrm{A}=$ $165.4, \mathrm{a}=2.1$ (for $\mathrm{x}$ expressed as a proportion of basilar membrane length), and $\mathrm{k}=1.0$. The values for a and $k$ vary for different species (Greenwood, 1990).

$$
\mathrm{F}_{\mathrm{Hz}}=\mathrm{A}\left(10^{(\mathrm{ax})}-\mathrm{k}\right)
$$

Consequently, curves derived from Greenwood's equations parallel the membraneelasticity curves obtained by von Békésy. Estimates of overall hearing ranges based on either cochlear length or length and width were also reported by West (West, 1985) and Manley (Manley, 1971). Fay's (1992) extrapolation of Greenwood's work shows that estimators derived from even a single basilar membrane dimension provide very close approximations of psychophysical measures of hearing for most land mammals. For these species, called generalists, there is an exceptionally close-fit of electrophysiological response data with predicted position-frequency maps for the same species (Fay, 1992). Fay's work (1992) shows that the basilar membrane frequency (BMF) equation can be used to derive estimates of critical bands (CB), critical masking ratio (CRB), and frequency discrimination thresholds (FDT) that are comparable to psychophysical values for any generalist land mammal ear (Fay, 1992; Echteler et al., 1994). 
However, these formulae provide a poor fit to hearing curves of species with specialized hearing [e.g. horseshoe bat (Rhinolophus ferrumequinum) and the mole rat (Spalax ehrenbergi)] (Echteler et al., 1994). Measurements of the relative stiffness of the basilar membranes of these species reveal differences in the stiffness gradient of the basilar membrane from the generalist basilar membrane. Species with high frequency hearing tend to have relatively narrower and thicker basilar membranes and the outer spiral laminae are more developed and continue through most of the cochlear duct. In species with ears specialized for low frequency hearing, the basilar membrane is generally wider and thinner, with the outer spiral laminae thinner and present only in the basal region of the cochlea (Echteler et al., 1994).

The ability to predict hearing capabilities from observable cochlear morphology would be very useful, particularly for species that are not amenable to traditional psychoacoustic studies such as baleen whales. For generalist species, correlations have been reported for the octave range of hearing and number of cochlear turns and basilar membrane length, with more turns or longer membrane length correlated with an increased range of hearing (Echteler et al., 1994). These correlations are not found in specialized cochleae and may not have any functional significance, as basilar membrane length actually scales with body size and not with hearing range (Ketten, 1984). However, measurements of basal and apical stiffness of the basilar membrane, which can be approximated by the ratio of thickness to width, seem to predict the upper and lower frequency limits equally well for both generalists and specialist ears (Echteler et al., 1994).

\subsubsection{Hearing studies in cetaceans}

Most hearing data from cetaceans come from studies with small captive odontocetes. Auditory threshold measurements can be made for these species using both behavioral and electro-physical techniques, such as auditory brain stem responses (ABR). Audiograms have been made from ten of these species since the first dolphin audiogram 
was published in the late 1960's (Au, 2000). The audiograms indicate similarities in all these odontocetes, with a wide range of frequency sensitivity up to at least $100 \mathrm{kHz}$ in all species. All of the species that have been tested echolocate. Similarities in hearing between bats and odontocetes may be related to this shared trait.

There have been no direct measures of hearing in mysticetes. All current estimates of mysticete hearing are from indirect evidence. First, the frequency range of best hearing is thought to coincide with that of the calls produced by the whales. Playback experiments with several species have documented behavioral responses to conspecific calls (Clark \& Clark, 1980; Watkins, 1981; Tyack, 1983). Playback experiments have also shown response of gray whales (Eschrichtius robustus) to the calls of killer whale predators (Orcinus orca) (Cummings \& Thompson, 1971). One playback trial estimated the received level of sound necessary to elicit an approach response from humpback whales (Megaptera novaeangliae) (Frankel, 1995). Additional studies of mysticete response to anthropogenic noise sources documented response at higher frequencies, up to at least $15 \mathrm{kHz}$ (Watkins, 1986). Also, playback experiments have demonstrated directional hearing capabilities in mysticetes by showing precise orientation toward and localization of the sound source (Clark \& Clark, 1980; Tyack, 1983; Parks, 2003). Ambient noise levels may limit the real world detection threshold for baleen whales. Baleen whale vocalizations have most of their energy below $1 \mathrm{kHz}$. The average ambient noise levels in all $1 / 3$ octave bands below $1 \mathrm{kHz}$ are higher than $75 \mathrm{~dB}$ re $1 \mu \mathrm{Pa}$ (Urick, 1983).

\subsubsection{Anatomical modeling for marine mammal hearing}

Comparative anatomical studies of cetacean and terrestrial mammalian ears have resulted in the discovery of particular adaptations unique to cetacean ears (Ketten, 1992). In both cetacean groups (odontocete and mysticete), the external pinna and outer ear canal were lost and the middle and inner ear shifted to a position outside the skull. In baleen whales the middle and inner ear bone have a direct connection to the skull while in 
odontocetes little or no bony connection remains (Ketten, 2000). In both groups, the ear bone consists of two connected bullae, consisting of the tympanic and periotic bones (Ketten, 2000).

In many other respects, odontocete and baleen whales differ in their hearing anatomy. In odontocetes, specialized fatty tissue along the lower jaw seems to function as an analog of external pinnae by channeling sound directly to the ear (Ketten, 1994). In baleen whales, the method of sound transduction remains unclear although conduction of sound through bone has been suggested (Ketten, 2000). The remaining difference in odontocete and baleen whale ears stems from the specialization of odontocetes for high frequency hearing. The odontocete ossicular chain shows evidence of stiffening elements similar to those seen in bats (Ketten, 1992). In baleens, the ossicles are larger and loosely joined, consistent with low frequency hearing (Ketten, 2000).

Cetacean basilar membranes range $18 \mathrm{~mm}$ to $75 \mathrm{~mm}$ in length (Ketten, 1992; Ketten, 2000). Basilar membrane lengths in Cetacea, like those of terrestrial mammals, scale with body size. The length of the basilar membrane is not significantly correlated with frequency characteristics. The thickness and width of the membrane are highly correlated with the frequency range of hearing because they relate to the stiffness gradient of the membrane. These thickness-to-width ratios are the most reliable correlate of frequency for cetacean basilar membranes (Ketten, 1994). In most odontocetes (except the sperm whale (Physeter catodon), basilar membrane width is narrow at the base $(30-50 \mu \mathrm{m})$ and increases to $300-500 \mu \mathrm{m}$ at the apex (Ketten, 2000). The basal widths of odontocete membranes are similar to those of bats at the basal end (30-50 $\mu \mathrm{m})$ and closer to humans at the apical end $(504 \mu \mathrm{m})($ Ketten, 2000). In mysticetes, the basilar membranes are wider and thinner than those of odontocetes, ranging from $100 \mu \mathrm{m}$ at the base to 1,500-2,200 $\mu \mathrm{m}$ at the apex (Ketten, 1992; Ketten, 2000). The basal width in mysticetes is similar to that of humans $(150 \mu \mathrm{m})$ and is $2-3$ times wider than in odontocetes. The apical width in baleen whales is much wider than reported for any species other than African elephants, which are known to perceive infrasonics (Payne et al., 1986). Based on width alone, odontocete and baleen whale basilar membranes are 
highly differentiated structures capable of wide, but very different, ranges of frequency response.

We know now that single parameter formulae, such as Greenwoods' equation which uses membrane length (Greenwood, 1961; Greenwood, 1962; Greenwood, 1990), are insufficient to describe cetacean ears (Ketten, 1984; Ketten, 1992; Echteler et al., 1994). While whale ears have the same basic components as land mammal ears, they also have adaptations to the aquatic environment that require more comprehensive modeling. Generalized morphometric models for land mammals provide a procedural or mechanistic basis for marine mammal analyses but we must modify them to accommodate structural differences in whale ears compared to those from typical land mammals. In particular, whale basilar membranes violate the fundamental assumption of generalist models, which is that stiffness and mass covary consistently with length.

Whale ears have stiffness variations inconsistent with generalist models. Because whale ear stiffness and mass scale differently than land mammal ears, estimates using generic land mammal formulae are incorrect (Ketten, 1984).

Frequency ranges and peak spectra can be reliably predicted for any mammal ear using a combination of several inner ear measurements (Ketten, 1984; Ketten, 1992). The appropriateness of a more comprehensive model for whales was first demonstrated in a structural analysis of the cochlea of 12 odontocete species (Ketten, 1984; Ketten \& Wartzok, 1990). In these studies, both single and multiple parameters were used to predict hearing range. Predictions using one to four anatomical parameters were compared with behavioral audiograms in four well-documented cetacean species. As more parameters are included, basilar membrane models become more comprehensive and substantially more accurate. The results of these studies showed that a combination of four measurements of cochlear structure (basilar membrane dimensions, laminar extent, membrane pitch and basal turn ratio) allowed for excellent prediction of the primary bands of ultrasonic hearing in odontocetes (Ketten, 1984; Ketten \& Wartzok, 1990). 
A definitive estimate of the hearing frequency range for any one species requires analyses of the morphometrics of the cochlea and basilar membrane. From these, proportional stiffness gradients and hearing ranges can be accurately calculated. In this study, measurements of membrane pitch, basal turn ratio, and basilar membrane dimensions are used to estimate the hearing frequency range for the North Atlantic right whale. These results represent a substantial and significant increase in our knowledge and understanding of baleen whale hearing by addressing hearing in this highly endangered species.

\subsection{METHODS}

\subsubsection{Specimen collection}

The endangered status of the North Atlantic right whale has resulted in extraordinary efforts to determine the cause of death in all known mortalities. This has resulted in the retrieval of carcasses many miles offshore, as well as carcasses that wash up on beaches. The high level of monitoring of the population and the positive buoyancy of right whales often result in sighting of right whales shortly after death. Although the thick blubber layer of right whales seems to increase the rate of decomposition due to increased core temperature, these efforts have resulted in the retrieval of high quality specimens that would not otherwise be possible. This has resulted in modern morphometric and anatomical data collected for this species that is unrivaled by any unharvested baleen whale population.

Right whale temporal bones that have been routinely collected from necropsies since 1990 reside in the Ketten laboratory at the Woods Hole Oceanographic Institution. The majority of specimens used in this study were obtained prior to 1998. Three ear specimens were collected during the duration of this project (EG18, EG19, EG20).

\subsubsection{Computerized Tomography (CT)}


Each specimen in the collection was imaged using computerized tomography (CT) scanning. The extended duration of the study resulted in several different scanners and protocols being used in the imaging process. The specimens were scanned using an ultra-high resolution protocol on a Siemens Spiral Plus 4 CT located at the Massachusetts Eye \& Ear Infirmary or on a Siemens Emotion Spiral CT and Volume Zoom scanners in the Ocean Imaging Center at the Woods Hole Oceanographic Institution by D. R. Ketten or J. Arruda. Spiral scans were made at $1 \mathrm{~mm}$ resolution, with $0.5 \mathrm{~mm}$ reconstruction of the 3D images. The exceptions to this were specimens EG 4, 5 and 18 which were scanned at $0.5 \mathrm{~mm}$ slice resolution. These scans provided 3D imaging of the right whale ears (Figure 5.1-5.3) as well as 2D imaging of the interior of the cochlea (Figure 5.4b).

The complete ear bone complex consists of the tympanic bone and the periotic bone in a "tympano-periotic complex" (Ketten, 2000) (Figure 5.1). The tympanic bone is a large dense shell-shaped bone that contains the ossicles and the middle ear space. The cochlea is contained within the periotic bone (Ketten, 2000). The two bones are joined together on the lateral and posterior sides by two bony projections. The tympano-periotic complex is wedged into the skull by flanges, two bony projections from the periotic bone. The posterior flange has been removed from the specimen shown in Figure 5.1 but can bee seen in Figure 5.10.

Number of turns in each cochlea

The number of turns in each individual cochlea was counted by making 3D reconstructions of the cochlear duct. The 3D images were oriented for a top-down view of the apical end of the cochlea to determine the rotation angles (Figure 5.2B). Figure $5.2 \mathrm{~B}$ illustrates a cochlea with 2.3 turns. The number of turns are counted from the base of the spiral (shown on the right edge of Figure 5.2B) to the apex. 

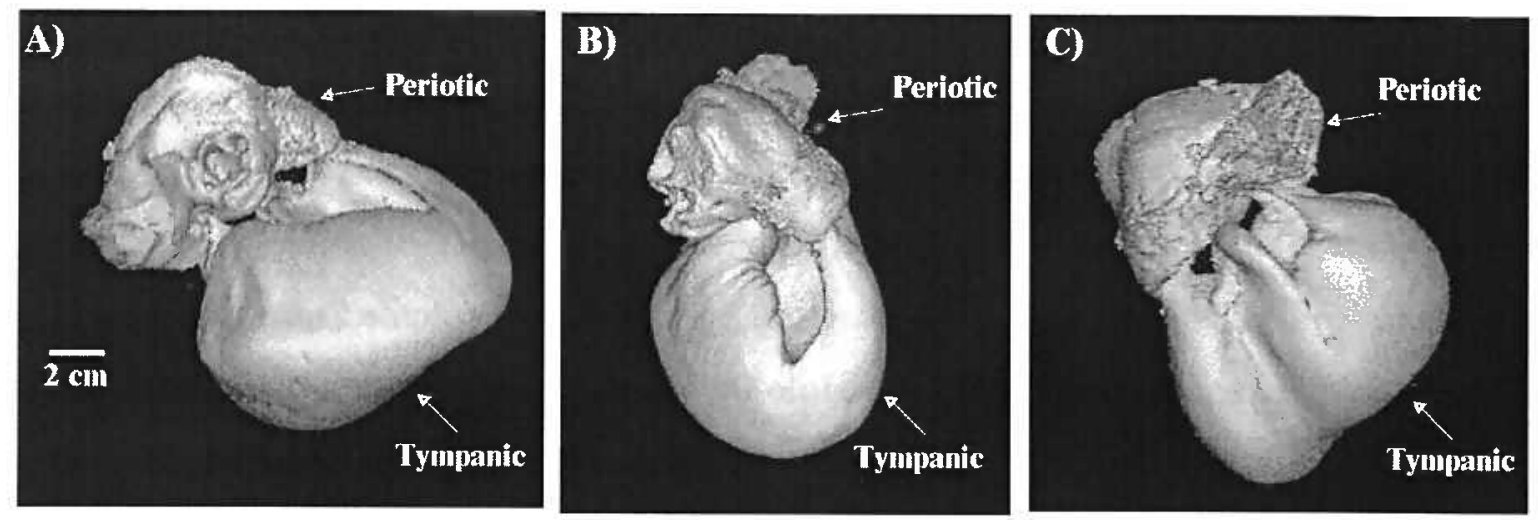

Figure 5.1. 3-D reconstruction of the entire left temporal bone complex from specimen EG5. The reconstruction is from $0.5 \mathrm{~mm}$ sections CT scans. The tympanic and periotic are labeled in each image. A) Medial view $B$ ) Anterior view C) Lateral view.
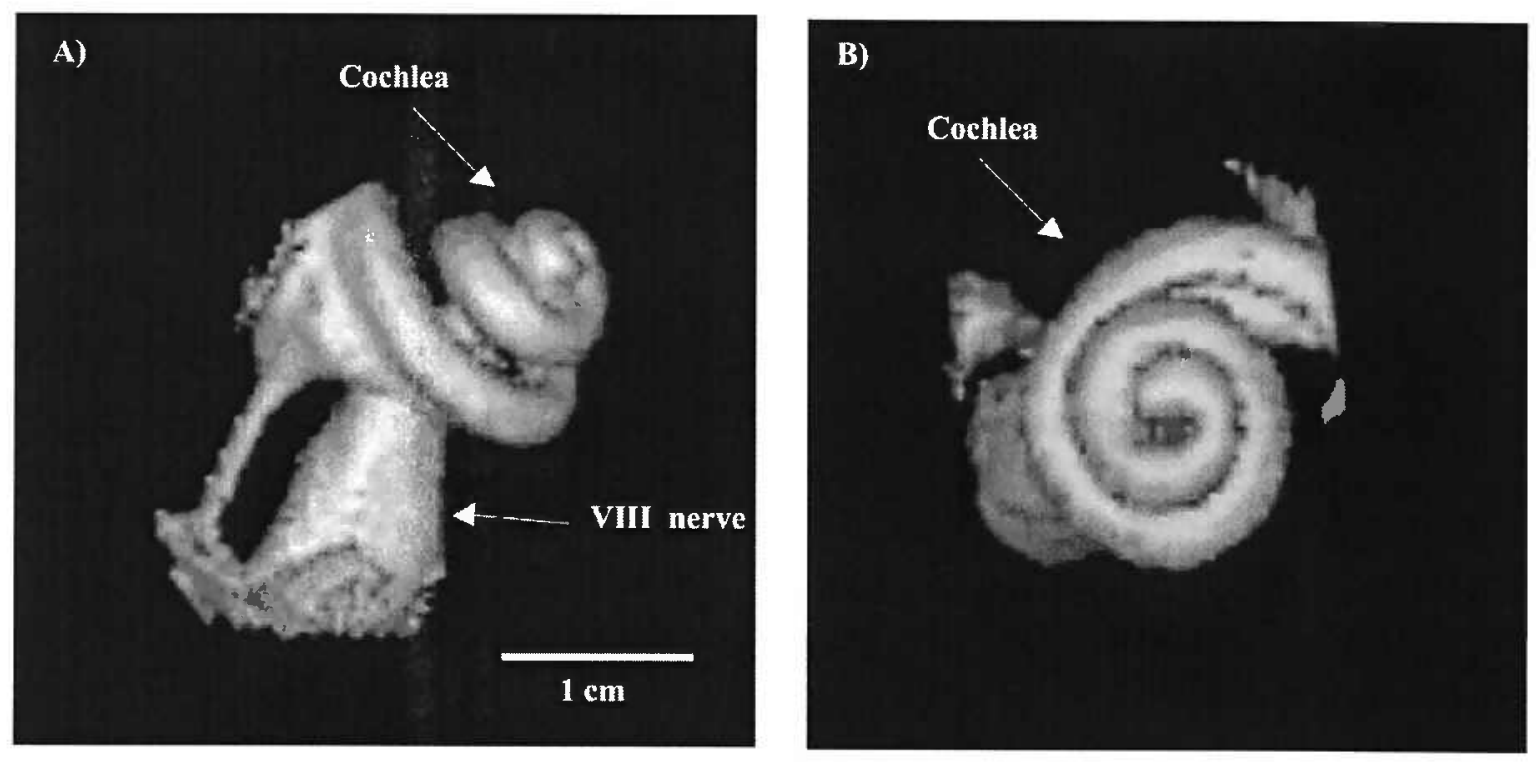

Figure 5.2. 3-D reconstruction of the cochlea from specimen EG 6 A) lateral view showing the height of the right whale cochlea spiral and the VIIIth nerve B) View of the cochlear spiral illustrating the number of turns in the right whale cochlea. 


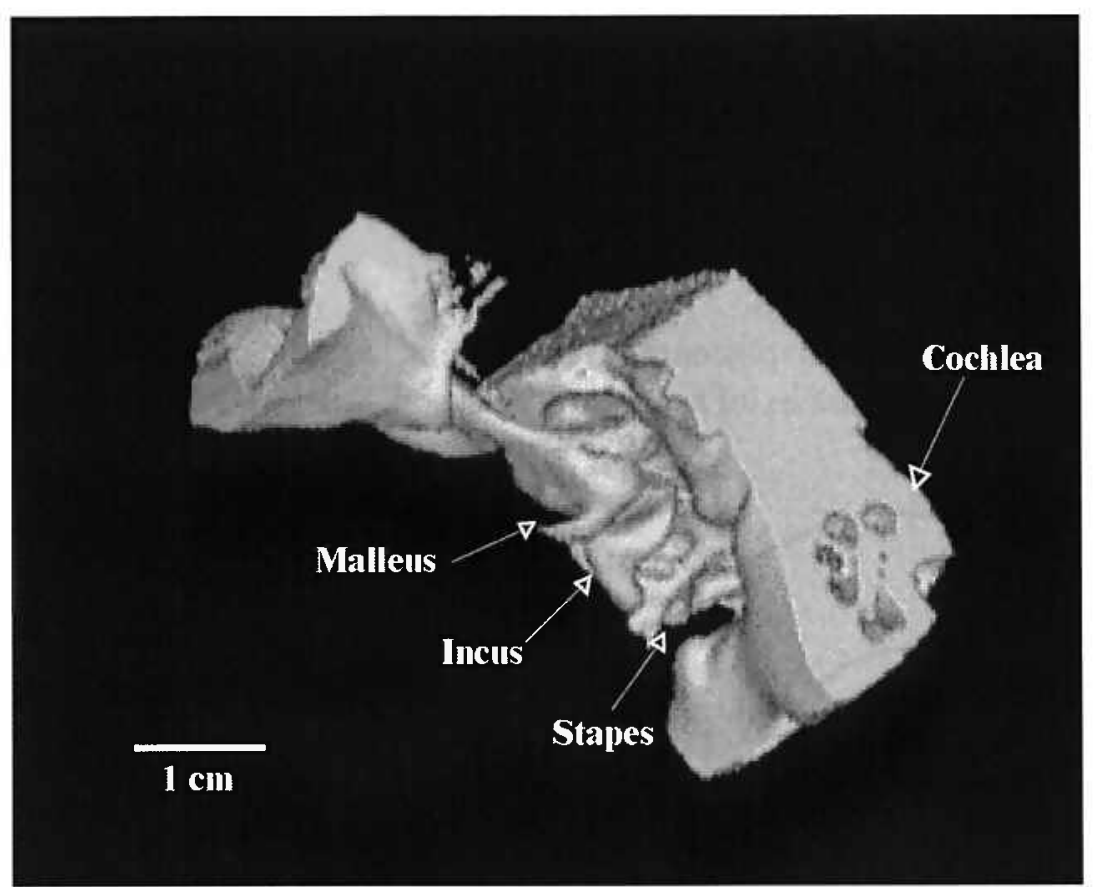

Figure 5.3. 3-D reconstruction of the ossicular chain from a right whale. The figure shows the in situ orientation, the angle between ossicles, as well as the position of the ossicles relative to the cochlea. A cross section through the cochlea can be seen on the right side of the figure. This cross section is approximately the same orientation as the cross-section shown in figure 5.4.

\section{Cochlear length estimation}

Cochlear lengths were determined by measuring the length of cochlear turn radii for multiple positions in the cochlea. CT scans were made from all preserved ear specimens in the collection. Cross sections through each cochlea were made at $1 \mathrm{~mm}$ slices, reconstructed at $0.5 \mathrm{~mm}$ intervals. The orientation of the CT cross-sections used for the measurements is shown in figure 5.4. This orientation, which is paramodiolar, was selected to make consistent measurements between ears. The slices were made perpendicular to the long axis of the basal turn because the basal turn is more ovoid than round in shape. Figure 5.5 shows the measurements made for each specimen. These values were then used to calculate the axial pitch, basal ratio, and the length of the cochleae. The length calculations were made using the following formulae (Ketten et al., 1998): 
(2)

$$
\text { For } r=a \theta
$$

$$
z=\sqrt{\left\{a / 2\left[\left(\theta \sqrt{\theta^{2}+1}\right)+\operatorname{Ln}\left(\theta+\sqrt{\theta^{2}+1}\right)\right]\right\}^{2}+h^{2}}
$$

Where $\mathrm{r}=$ radius at angular displacement $\theta$ in radians. $\mathrm{a}=$ constant that determines the size of the spiral, and $h$ is the axial height of the spiral.

A)

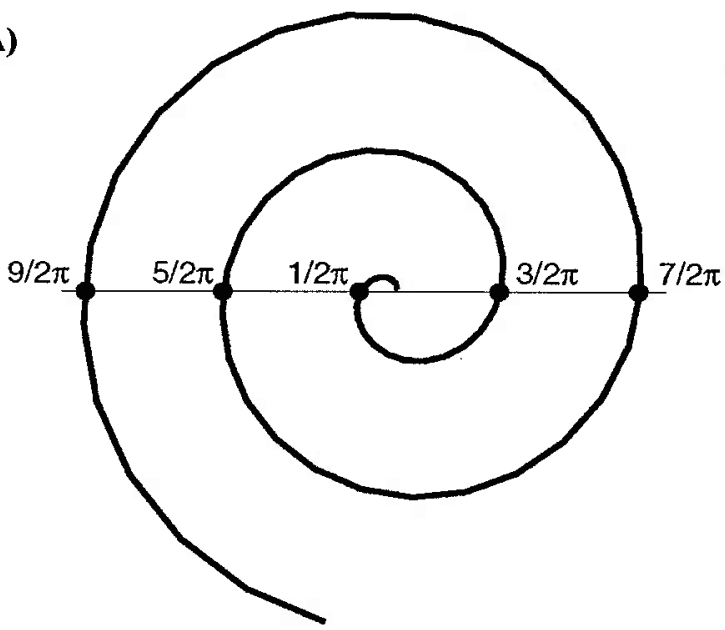

Figure 5.4. Cross section through a right whale cochlea for CT measurements. A) Schematic showing the orientation of the cross section B) An example of the resulting 2D CT image from the cross section.

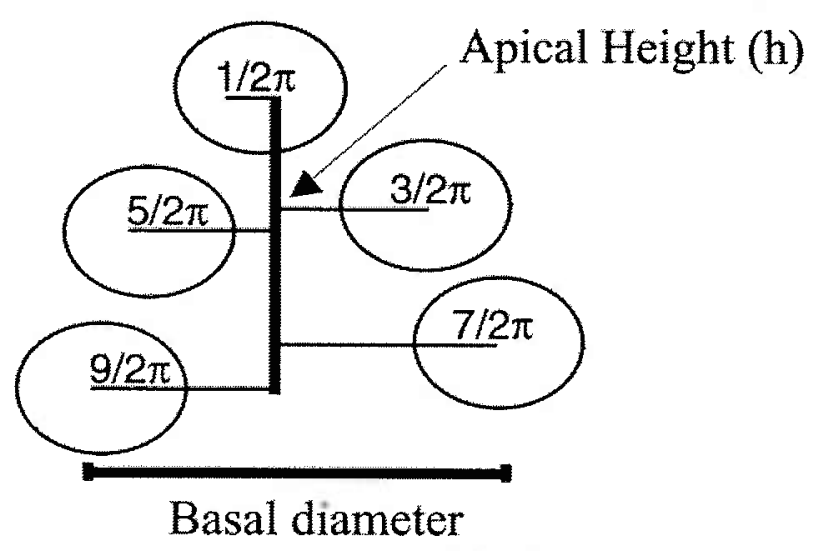

Figure 5.5. Illustration of the measurements made from $2 \mathrm{D}$ CT cross sections from right whale specimens with 2.5 turns.

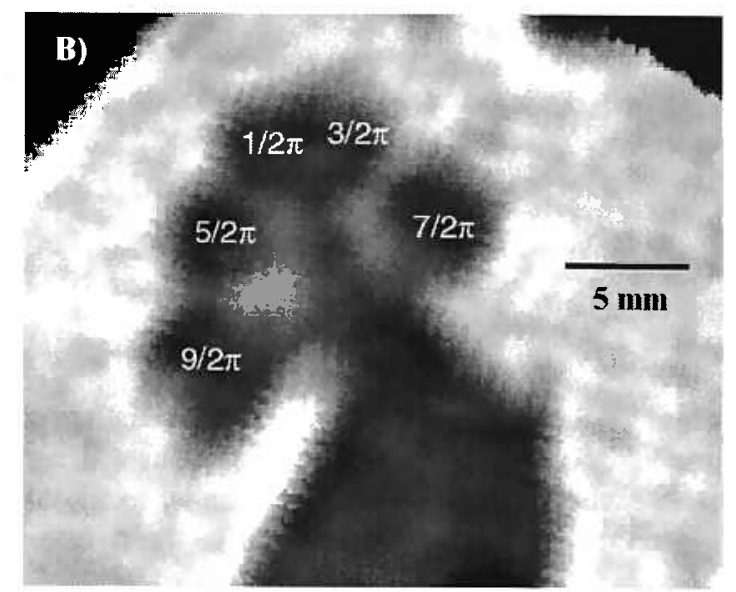




\section{Ground truthing cochlea length calculations}

3-D reconstructions of each cochlea were measured to compare the observed cochlear length from the CT scans with calculated cochlear length based on radii measurements. The basal diameter of the cochlea was measured from the 3D reconstructions in the same orientation that the radial cross-sections were taken. These values were used to scale the measurements based on the measurement of basal diameter made from the cross-section shown in Figure 5.5. These estimates are rough approximations of cochlear length as they do not take into account the rise of the spiral.

\subsubsection{Gross Dissection}

Specimens with acceptable preservation of the VIIIth nerve and the basilar membrane based on the CT scan images were selected for further processing. These specimens were dissected, which involved defrosting frozen specimens in formalin and removal of all remaining external soft tissue. The remaining tissues were weighed to give approximate mass for the entire ear complex. Then the periotic and tympanic bones were separated and the bony flanges were removed from the periotic by use of a handsaw to reduce the volume of bone surrounding the cochlea. The periotic and tympanic bones were also measured for length/width and weighed. When present, ossicles were removed and preserved in a $1 \%$ formalin solution for use in density measurements at a later date.

\subsubsection{Histology}

The periotic bones from the gross dissection were placed into solution to decalcify the hard tissue surrounding the cochlea to allow sectioning for slides. The ears were decalcified in either $5 \%$ trichloroacetic acid with later transfer to EDTA or in acid or EDTA only. One specimen (EG 18) was decalcified in acid only. Bone wax was placed in the oval and round windows of this specimen to reduce the impact of the acid on the 
inner tissues of the cochlea. The mass and density of baleen whale ears requires long periods of time for decalcification. For example, a periotic bone decalcified in EDTA takes 18-24 months. Therefore, acid decalcification of various durations were attempted to determine if this method is acceptable and to find the effects of acid techniques on inner ear structures, especially the cochlear duct.

After decalcification was complete, the ears were embedded in celloidin solution to harden and sectioned into $20 \mu \mathrm{m}$ sections. These sections were stained with hematoxylin and eosin and mounted onto slides by J. O'Malley.

\section{Basilar membrane measurements}

The preservation of the cochlear duct varied in the specimens sectioned for slides. All specimens had measurable intact membranes in part of the cochlea (Figure 5.6). However, all specimens had regions where the basilar membrane was broken or disintegrated from decalcification or post-mortem decay. The wider, thinner membranes near the apical turn of the cochlea were the most commonly lost, while the shorter, thicker membrane supported by the outer osseous spiral laminae was intact for all specimens. Figure 5.13 illustrates this point. Note that the basilar membrane is only intact in the basal turn for EG1, EG4 and EG9. The apical turns of the cochlear duct are completely acellular in three specimens.

Basilar membranes that were present in the slide sections were measured for width and thickness (Figure 5.6). The width was measured at a 40x objective magnification on a light microscope (Olympus Model BX40) with a graticule and ocular (10x) calibrated scale used for measurements. The basilar membrane was so thin in some areas that in many cases oil immersion microscopy using a $100 \mathrm{x}$ oil immersion lens was necessary to measure basilar membrane thickness. Measurements for width and thickness were recorded for all intact portions of the basilar membrane from all slides. 


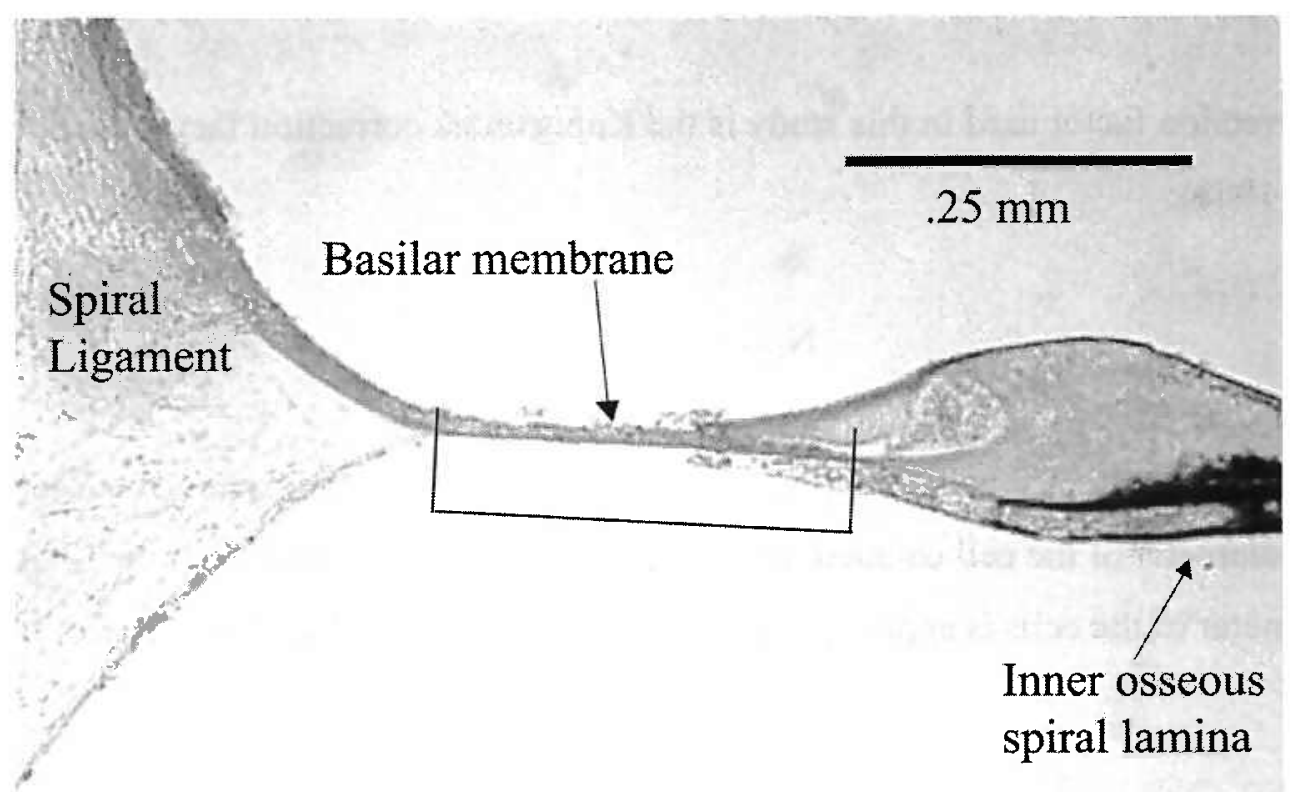

Figure 5.6 Basilar membrane from EG9 at 20X marking points for measurement of the width of the membrane. The thickness of the membrane was measured in the center of the membrane.

\section{Ganglion cell counts}

Ganglion cells were counted from each slide from all four specimens (Figure 5.7). The count was made in each visible turn of the cochlea and total number of ganglion cells and total number of ganglion cell nuclei were counted at $40 \mathrm{x}$ resolution using a grid. To estimate the total number of ganglion cells and to plot them on the reconstruction of the basilar membrane, the total number of visible cells was used. This is different than the convention in human studies in which only the total number of ganglion cells containing nuclei are used for counts. However, the thickness of sections in the right whale ears is approximately the same as the apparent size of the ganglion cells $15-25 \mu \mathrm{m}$. The number of ganglion cells counted from the mounted slide sections were multiplied by 10 (to account for the unmounted sections) (Schuknecht, 1993). A correction factor is necessary because of the statistical likelihood that a cell could be split between sections, which would result in double counting of cells. 
The correction factor used in this study is the Konigsmark correction factor described in Nadol (1988):

$$
\mathrm{N}_{\text {corr }}=\mathrm{N}_{\text {coun }}(\mathrm{t} / \mathrm{t}+\mathrm{d})
$$

Where $\mathrm{N}_{\text {corr }}=$ corrected cell count, $\mathrm{N}_{\text {coun }}=$ actual cell count, $\mathrm{t}=$ thickness of the section and $d=$ diameter of the cell counted. In this case, the thickness of the section $=20 \mu \mathrm{m}$ and the diameter of the cells is approximately $20 \mu \mathrm{m}$, leading to a correction factor of 0.5 .

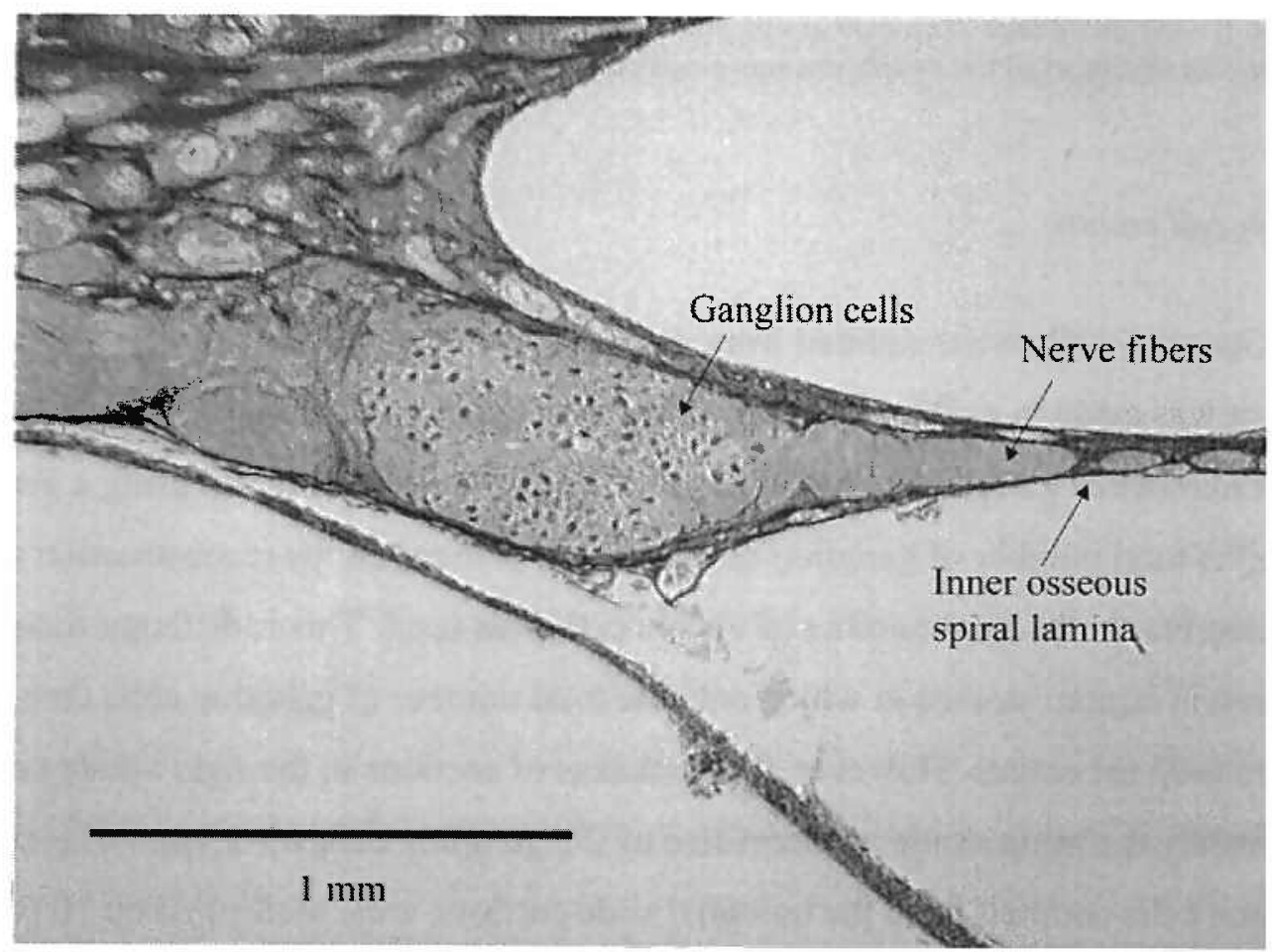

Figure 5.7. Ganglion cells from the basal turn of EG4. The ganglion cells in all ears showed evidence of cell loss, and all ganglion cells had a collapsed appearance. The nerve fibers connecting to the hair cells and the inner osseous spiral lamina are also labeled.

All four specimens show signs of decomposition and loss of ganglion cells. Therefore, combined counts pooling the highest ganglion cell counts for a given basilar membrane 
position from all specimens were used to get a better estimate of the total number of ganglion cells present in a right whale ear. The ganglion cell density/mm was calculated as a percentage of the basilar membrane length for the two specimens with the best preservation (EG 4 and EG 9). The density counts from these two specimens were also pooled to get a better estimate of the density of ganglion cells per unit length of the basilar membrane.

\subsubsection{Modeling of hearing range}

Using the measured thickness and width of the basilar membrane at multiple positions along its length, the absolute and functional hearing range of the right whale can be estimated. The absolute hearing range is a theoretical value, the total range of frequencies that the basilar membrane can respond to. The functional hearing range is generally somewhat narrower than the total hearing range. D.R. Ketten calculated the values of the estimated of hearing range for the North Atlantic right whale using the basilar membrane measurements provided in this study using the model described in Ketten (1994). 


\subsection{RESULTS}

A total of eighteen ears were analyzed from 13 different individuals. All ears were CT scanned, and four ears were further processed into slides for histology.

\subsubsection{Specimens}

Table 5.1. A list of all specimens measured for this study indicating the date of the stranding, the age of the whale at time of death, sex, total body length measured from snout to fluke notch, state of preservation of the specimen at time of necropsy(1-Best, 4-Worst), cause of death if known, and whether the specimen was analyzed using $\mathrm{CT}$ or further processed for histology. Full necropsy records for each specimen are available at the New England Aquarium and the Ketten Laboratory at the Woods Hole Oceanographic Institution. Four of the specimens (EG1, EG4, EG9, and EG18) were sectioned into slides. *- Indicates that both ears were analyzed from these specimens.

\begin{tabular}{|c|c|c|c|c|c|c|c|c|c|}
\hline Specimen & Stranding & Age & Sex & $\begin{array}{l}\text { Length } \\
(\mathrm{cm})\end{array}$ & Code & Preservation & $\begin{array}{c}\text { Cause of } \\
\text { death }\end{array}$ & $C T$ & Slides \\
\hline Egl & $1 / 3 / 1989$ & Calf & $\mathbf{M}$ & 425 & 2 & Formalin & Unknown & $\sqrt{ }$ & $\sqrt{ }$ \\
\hline $\mathrm{Eg} 4 / 5^{*}$ & $2 / 22 / 1996$ & Calf & $\mathrm{M}$ & 407 & 3 & Formalin & Unknown & V & V \\
\hline $\operatorname{Eg} 6^{*}$ & $1 / 2 / 1996$ & Calf & $\mathrm{F}$ & 478 & 3 & Formalin & Unknown & $V$ & \\
\hline $\operatorname{Eg} 7 *$ & $7 / 17 / 1995$ & $21 / 2$ & $\mathrm{M}$ & 1030 & 3 & Formalin & Entanglement & $V$ & \\
\hline Eg8 & $1 / 30 / 1996$ & Adult & $\mathrm{M}$ & 1415 & 3 & Formalin & Vessel strike & $\sqrt{ }$ & \\
\hline Eg9 & $2 / 19 / 1996$ & Calf & $\mathrm{F}$ & 513 & 3 & Formalin & Unknown & $V$ & $\sqrt{ }$ \\
\hline Eg10 & $10 / 29 / 1999$ & 10 & F & 1350 & 4 & Frozen & Entanglement & $V$ & \\
\hline Eg11 & $3 / 9 / 1996$ & $\begin{array}{c}4+ \\
\text { years }\end{array}$ & $\mathrm{M}$ & 1270 & 3 & Frozen & Vessel strike & V & \\
\hline $\operatorname{Eg} 13 / 14$ & $1 / 9 / 1997$ & Calf & $\mathrm{M}$ & 417 & 3 & Formalin & Parturition & V & \\
\hline $\operatorname{Eg} 16^{*}$ & $8 / 19 / 1997$ & Adult & F & 1259 & 2 & Frozen & Vessel strike & $\sqrt{ }$ & \\
\hline Eg18* & $4 / 20 / 1999$ & Adult & $\mathrm{F}$ & 1370 & 2 & Formalin & Vessel strike & $\sqrt{ }$ & V \\
\hline Eg19 & $3 / 18 / 2001$ & Calf & $\mathrm{M}$ & 660 & 3 & Formalin & Vessel strike & V & \\
\hline $\mathrm{Eg} 20$ & $6 / 18 / 2001$ & Calf & $\mathrm{F}$ & 1050 & 3 & Frozen & Vessel strike & V & \\
\hline
\end{tabular}

\subsubsection{CT scan measurements}

Initial surveys of cochlear dimensions from CT images showed that precise orientation of the cross sections taken by the CT scanner are important for consistent measurement of the basal diameter of right whale cochleae. While the higher turns of the 
cochlea appear close to circular in their structure, the basal turn is more elliptical, which means the orientation of the scans used for basal measurement must be consistent. After initial measurement disregarding the orientation of the cross-section resulted in large $(20 \%)$ variations in radii measurements, the orientations for all specimens were standardized to the orientation shown in Figure 5.4. The resulting measurements are shown in Table 5.2.

Table 5.2. Measurements taken from the CT scans for all specimens. The radii $(1 / 2 \pi, 3 / 2 \pi, 5 / 2 \pi, 7 / 2 \pi$ and $9 / 2 \pi$ for $2.4-2.5$ turns, $0, \pi, 2 \pi, 3 \pi$, and $4 \pi$ for 2.25 turns), axial height and basal diameter are reported in $\mathrm{mm}$. The number of turns are counts made from 3-D reconstructions of the cochlea for each specimen. The axial pitch $=$ axial height/number of turns and the basal ratio $=$ axial height/basal diameter defined by Ketten 1984.

\begin{tabular}{l|cccccccccc}
\hline \multirow{2}{*}{ Specimen } & $1 / 2$ & $3 / 2$ & $5 / 2$ & $7 / 2$ & $9 / 2$ & Axial & Basal & Number & Axial & Basal \\
& $\pi$ & $\pi$ & $\pi$ & $\pi$ & $\pi$ & Height & Diameter & Purns & Pitch & Ratio \\
\hline EG5 Left & 1.23 & 2.18 & 2.80 & 3.49 & 4.69 & 5.54 & 8.18 & 2.50 & 2.22 & 0.68 \\
EG6 Right & 0.94 & 2.61 & 3.26 & 3.77 & 5.34 & 5.74 & 9.11 & 2.40 & 2.39 & 0.63 \\
EG6 Left & 0.96 & 2.20 & 3.48 & 3.77 & 5.23 & 5.77 & 9.00 & 2.40 & 2.40 & 0.64 \\
EG8 Right & 1.47 & 1.71 & 3.22 & 3.92 & 5.54 & 5.88 & 9.47 & 2.25 & 2.61 & 0.62 \\
EG10 Left & 0.99 & 2.26 & 2.99 & 4.06 & 4.87 & 5.68 & 8.93 & 2.50 & 2.27 & 0.64 \\
EG11 Right & 1.45 & 2.07 & 2.97 & 3.68 & 5.29 & 5.82 & 8.97 & 2.50 & 2.33 & 0.65 \\
EG9 Left & 0.98 & 2.24 & 3.00 & 3.56 & 5.48 & 5.85 & 9.04 & 2.50 & 2.34 & 0.65 \\
EG7 Right & 1.12 & 1.93 & 2.83 & 3.40 & 4.34 & 5.85 & 7.74 & 2.50 & 2.34 & 0.76 \\
EG7 Left & 1.19 & 1.85 & 2.87 & 3.41 & 4.27 & 5.91 & 7.69 & 2.50 & 2.36 & 0.77 \\
EG13 Right & 1.20 & 2.25 & 3.21 & 3.78 & 5.25 & 5.96 & 9.02 & 2.50 & 2.38 & 0.66 \\
EG16 Right & 1.03 & 2.24 & 2.64 & 3.70 & 4.77 & 5.61 & 8.46 & 2.50 & 2.24 & 0.66 \\
EG16 Left & 1.15 & 1.59 & 2.49 & 3.52 & 4.53 & 5.44 & 8.05 & 2.50 & 2.18 & 0.68 \\
EG18 Right & 1.26 & 2.16 & 2.77 & 3.51 & 5.02 & 6.26 & 8.53 & 2.50 & 2.51 & 0.73 \\
EG18 Left & 1.27 & 2.00 & 2.78 & 3.97 & 4.89 & 6.33 & 8.86 & 2.50 & 2.53 & 0.71 \\
EG19 Right & 1.11 & 2.02 & 2.86 & 3.35 & 4.67 & 5.54 & 8.02 & 2.25 & 2.46 & 0.69 \\
EG20 Right & 1.19 & 1.55 & 2.76 & 3.21 & 5.16 & 5.56 & 8.36 & 2.50 & 2.22 & 0.66 \\
\hline Average & $\mathbf{1 . 1 6}$ & $\mathbf{2 . 0 5}$ & $\mathbf{2 . 9 3}$ & $\mathbf{3 . 6 3}$ & $\mathbf{4 . 9 6}$ & $\mathbf{5 . 8 0}$ & $\mathbf{8 . 5 9}$ & $\mathbf{2 . 4 6}$ & $\mathbf{2 . 3 6}$ & $\mathbf{0 . 6 8}$ \\
SD & $\mathbf{0 . 1 6}$ & $\mathbf{0 . 2 8}$ & $\mathbf{0 . 2 6}$ & $\mathbf{0 . 2 4}$ & $\mathbf{0 . 3 9}$ & $\mathbf{0 . 2 5}$ & $\mathbf{0 . 5 4}$ & $\mathbf{0 . 0 9}$ & $\mathbf{0 . 1 2}$ & $\mathbf{0 . 0 5}$ \\
\hline
\end{tabular}




\subsubsection{Cochlear length}

The values of the radii were used to calculate the length of the cochlea using formulae $2 \& 3$ described in the methods (Ketten et al., 1998). The calculated cochlear lengths are shown in Table 5.3. Table 5.4 compares the calculated cochlear length with the actual cochlear length measured directly from a 3-D CT reconstruction. There was good agreement between the predicted and observed cochlear length for most specimens. However, the values measured from the CT images are slightly longer. The added length is likely a result of the inclusion of the terminal hook from the spiral in the measurements from the CTs.

Table 5.3. Measurements used for the calculation of length of the cochlear canal. Theta is the number of degrees in the spiral reported in radians. Axial height is the height of the spiral in $\mathrm{mm}$. The spiral constant a is calculated to give the relative size of the approximated spiral from equation 1. Cochlear length is the calculated $\mathrm{z}$ value from equation 2 .

\begin{tabular}{l|cccc}
\hline & Theta & Axial Height & $\begin{array}{c}\text { Spiral } \\
\text { constant }\end{array}$ & $\begin{array}{l}\text { Cochlear } \\
\text { length }(\mathrm{mm})\end{array}$ \\
\hline EG5 Left & 15.71 & 5.54 & 0.45 & 56.68 \\
EG6 Right & 15.08 & 5.74 & 0.53 & 61.68 \\
EG6 Left & 15.08 & 5.77 & 0.52 & 60.19 \\
EG8 Right & 14.14 & 5.88 & 0.56 & 57.03 \\
EG10 Left & 15.71 & 5.68 & 0.44 & 55.57 \\
EG11 Right & 15.71 & 5.82 & 0.43 & 53.71 \\
EG9 Left & 15.71 & 5.85 & 0.44 & 55.34 \\
EG7 Right & 15.71 & 5.85 & 0.42 & 52.98 \\
EG7 Left & 15.71 & 5.91 & 0.43 & 54.23 \\
EG13 Right & 15.71 & 5.96 & 0.47 & 59.46 \\
EG16 Right & 15.71 & 5.61 & 0.43 & 53.94 \\
EG16 Left & 15.71 & 5.44 & 0.41 & 51.06 \\
EG18 Right & 15.71 & 6.26 & 0.46 & 57.75 \\
EG18 Left & 15.71 & 6.33 & 0.46 & 57.88 \\
EG19 Right & $\mathbf{1 4 . 1 4}$ & 5.54 & 0.49 & 50.54 \\
EG20 Right & 15.71 & 5.56 & 0.42 & 52.69 \\
\hline Average & $\mathbf{1 5 . 4 3}$ & $\mathbf{5 . 8 0}$ & $\mathbf{0 . 4 6}$ & $\mathbf{5 5 . 6 7}$ \\
SD & $\mathbf{0 . 5 5}$ & $\mathbf{0 . 2 5}$ & $\mathbf{0 . 0 4}$ & $\mathbf{3 . 2 2}$ \\
\hline
\end{tabular}


Table 5.4. Comparison between the calculated cochlear length from CT radii measurements and the length of the cochlea measured directly from 3-D reconstructions of the cochlea.

\begin{tabular}{l|cc}
\hline Specimen & $\begin{array}{c}\text { Calculated } \\
\text { cochlea } \\
\text { length }(\mathrm{mm})\end{array}$ & $\begin{array}{c}\text { Measured } \\
\text { cochlea } \\
\text { length }(\mathrm{mm})\end{array}$ \\
\hline EG5Left & 56.68 & 53.94 \\
EG6Right & 61.68 & 62.90 \\
EG6Left & 60.19 & 63.03 \\
EG8Right & 57.03 & 58.89 \\
EG11Right & 53.71 & 55.17 \\
EG7Right & 52.98 & 56.14 \\
EG7Left & 54.23 & 54.26 \\
EG13Right & 59.46 & 63.16 \\
EG16Right & 53.94 & 55.24 \\
EG16Left & 51.06 & 53.56 \\
EG18Right & 57.74 & 59.69 \\
EG18Left & 57.88 & 56.62 \\
EG19Right & 50.54 & 52.11 \\
EG20Right & 52.69 & 52.82 \\
\hline Average & $\mathbf{5 5 . 7 0}$ & $\mathbf{5 6 . 9 7}$ \\
SD & $\mathbf{3 . 3 3}$ & $\mathbf{3 . 7 5}$ \\
\hline
\end{tabular}

\section{Reconstruction of cochlea}

Thickness and width measurements were reconstructed as shown graphically (Figure 5.8) to determine the exact position in the basilar membrane from which each measurement from a slide was taken (Figure 5.8). Each line represents the measured width of the basilar membrane at that position. The ganglion cell counts from each slide were also placed on the same map.

\subsubsection{Body size versus cochlear length}

Body length versus calculated cochlear length were plotted separately for adults and calves/juveniles. The calculated cochlear length correlated well with body length for adults (Figure 5.9a). The body size of calves did not correlate with cochlear length, as expected because they have not yet attained their total body length and because the cochlea is essentially mature at birth in mammals (Figure $5.9 \mathrm{~b}$ ). 


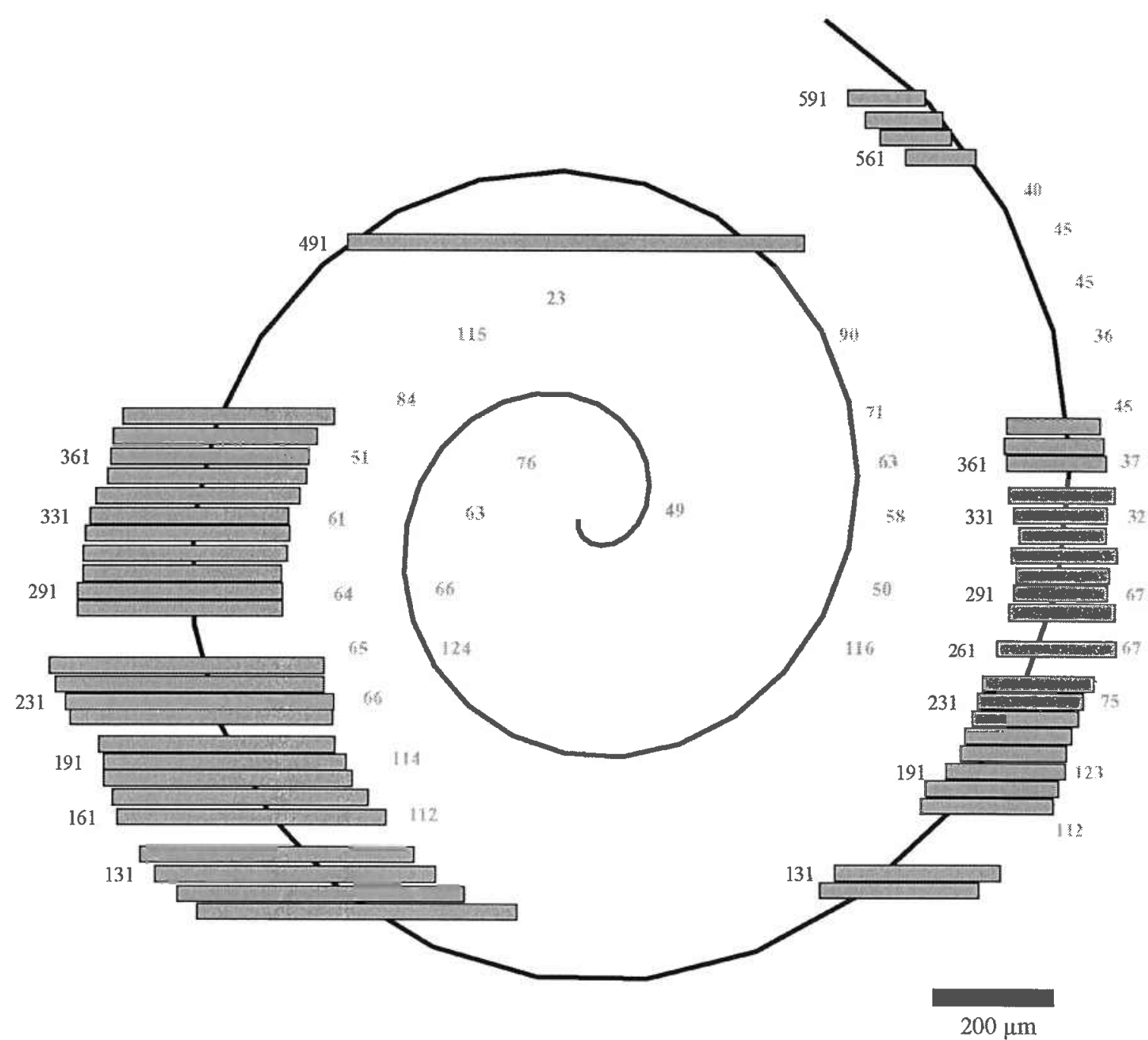

Figure 5.8. Image of a reconstructed basilar membrane from EG4 with ganglion cell counts from the slide measurements. The gray bars represent the length of the basilar membrane at particular points on the cochlea. The black numbers represent the slide number. The light gray numbers are the count of ganglion cells at each point on the corresponding slide. 
a)

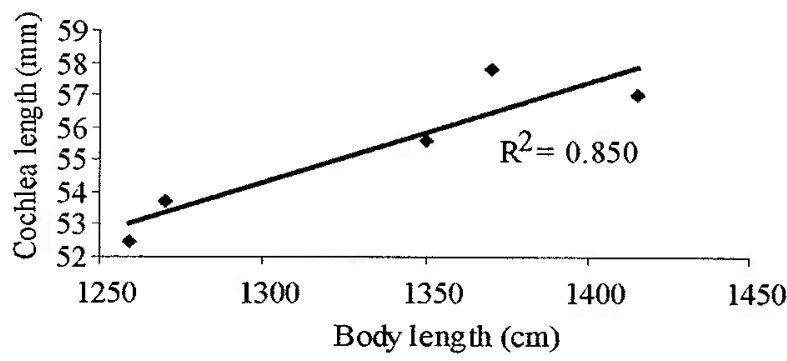

b)

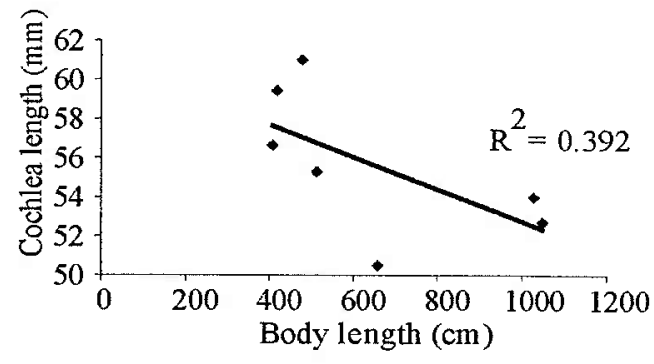

Figure 5.9. a) Body length (measured from snout to notch of flukes) versus calculated cochlear length for all adult animals. b) Body length versus cochlear length for neonates, calves and young juveniles. The $r-$ value is included in each plot.

\subsubsection{Gross dissection}

Ears with evidence on CT scans of preservation of the VIIIth nerve and the basilar membrane were dissected to separate the periotic from the tympanic and bony flange.

Figure 5.10 shows the complete temporal bone complex with all soft tissue removed.

Figure 5.11 shows the ossicles removed from one of the specimens.
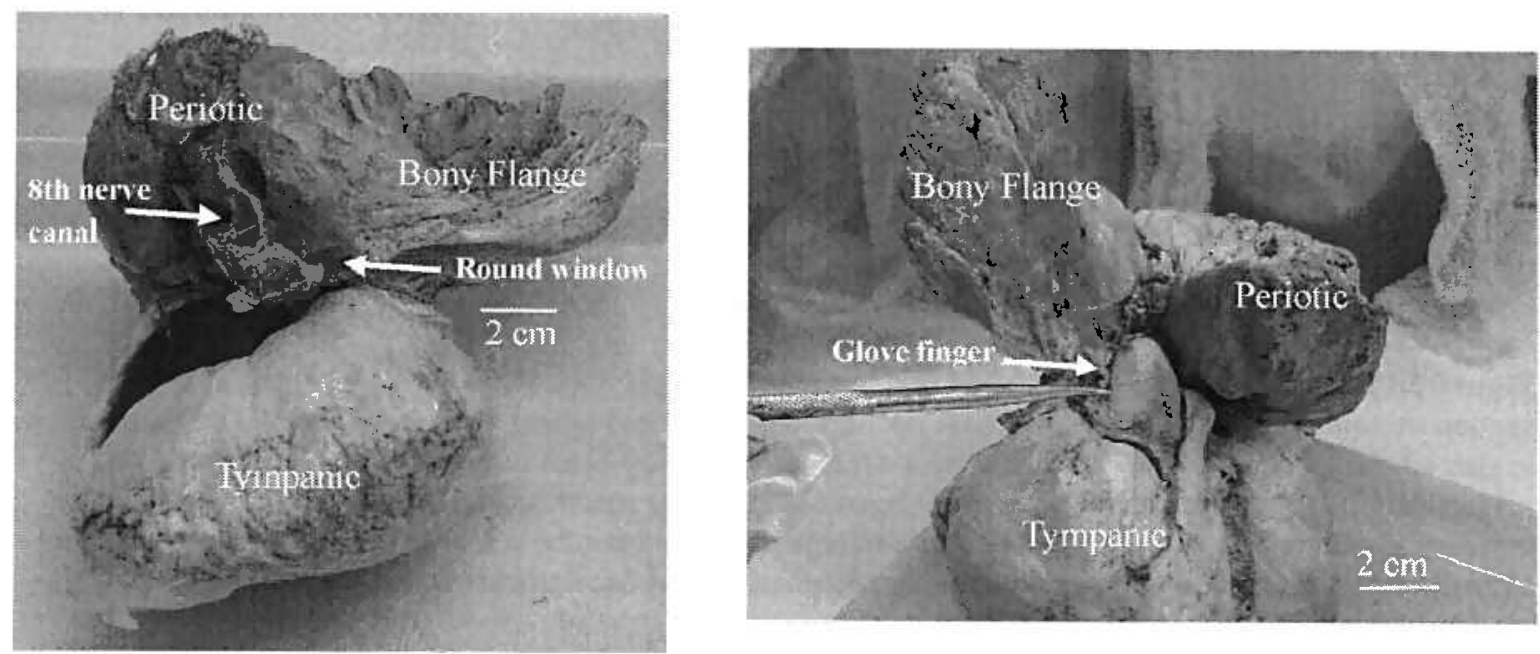

Figure 5.10. Images from dissection of EG11 temporal bones. A) The medial view of the right ear. The tympanic and periotic bones are labeled. The $8^{\text {th }}$ nerve canal, round window and bony flange are also labeled. B) The lateral view of the same ear. The tympanic, periotic and flange are labeled to aid in orientation. The glove finger, which is the common term for the baleen whale tympanic membrane is in its normal position between the two temporal bone elements. 


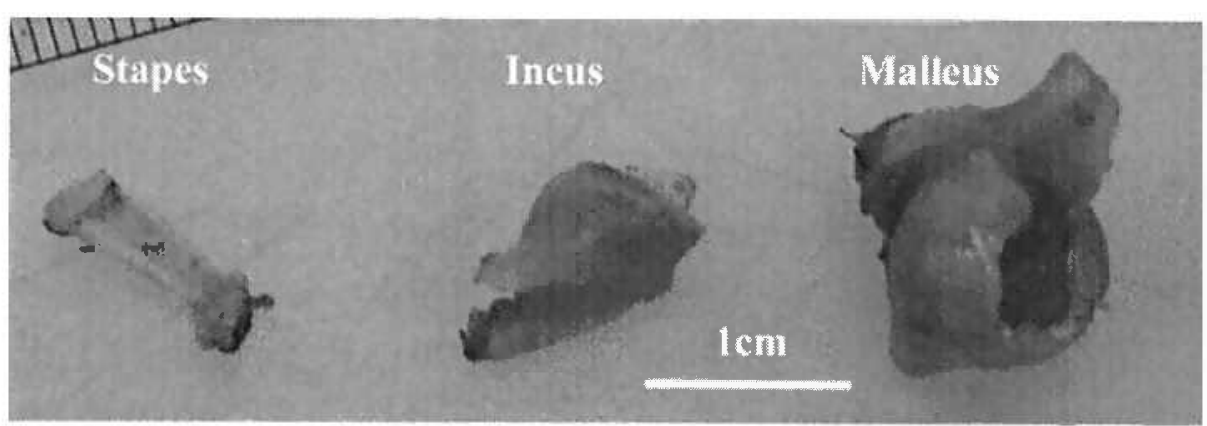

Figure 5.11. Ossicles taken from EG 20 ear during gross dissection illustrating the shape and size of right whale ossicles. The long arm of the malleus is missing in this photograph.

\subsubsection{Histology}

After decalcification the cochleae were sectioned and mounted on slides for measurement. Examples of the sections are shown in Figure 5.12.

a)

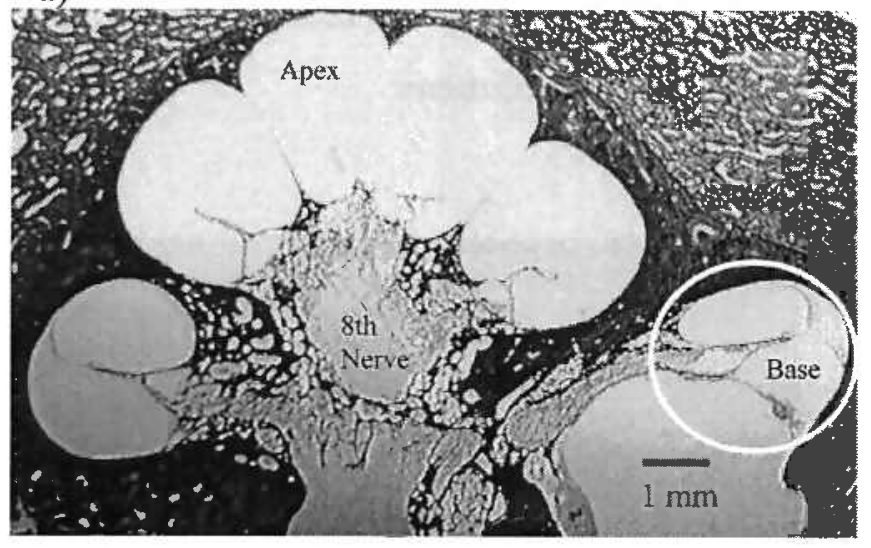

b)

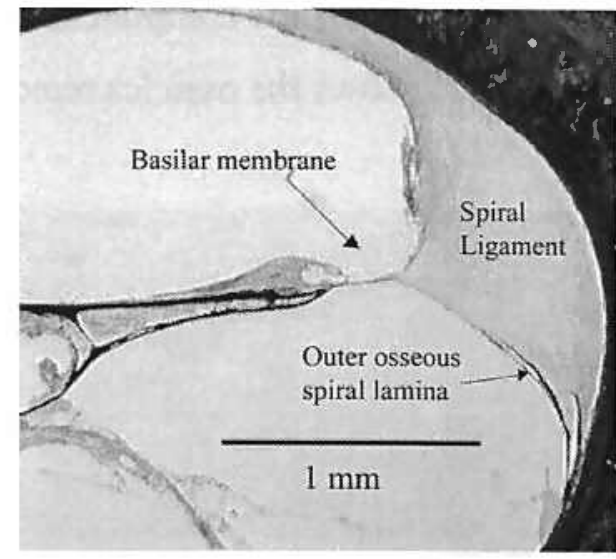

Figure 5.12. Images from histology slide preparations from specimen EG 9. a) A mid-modilar cross section showing the layout of a right whale cochlea. The basal and apical turns are labeled. The VIIth nerve is also labeled. The basal turn is marked by a white circle. b) The basal turn from a) under higher (15x) magnification. The basilar membrane, spiral ligament and outer osseous spiral lamina are labeled. Many inner ear structures are absent (e.g. Reissner's membrane separating the scala vestibuli and scala media) as a result of post-mortem changes and histology preservation and decalcification.

\section{Acid effects}

One specimen, EG 1 had previously been prepared as slides. The three ears processed for this work were all decalcified for varying periods of time in acid in an attempt to reduce 
the decalcification time and determine the effects of acid on the specimens. The acid decalcification appears to have resulted in varying degrees of artifact in the final sections. (Figure 5.13). Mid-modiolar cross sections through all four specimens show the impact of the acid decalcification. From Table 5.1, specimens EG1 and EG18 were in better condition at the time of dissection that EG4 and EG9 which were both moderately decomposed. Comparing the preparations of EG 1 (5.13A) and EG18 (5.13D) illustrates the effect of acid decalcification. No acid was used in the decalcification of EG1, and only acid was used for EG18. There is clear decalcification of the bone surrounding the cochlea from EG18 and all of the inner osseous spiral laminae have been dissolved, resulting in the breaking of any basilar membrane that may have remained when the specimen was collected.

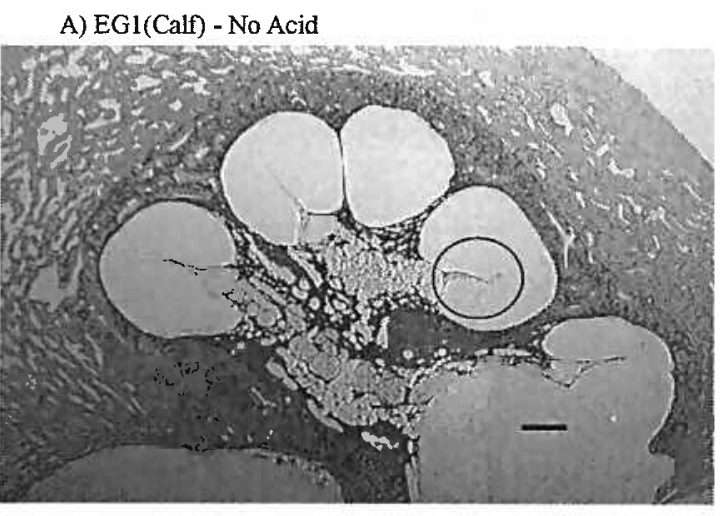

B) EG4 (Calf) - 2 Months in Tricholoracetic Acid

C) EG9 (Calf) - 1.5 Months in Trichloracetic Acid
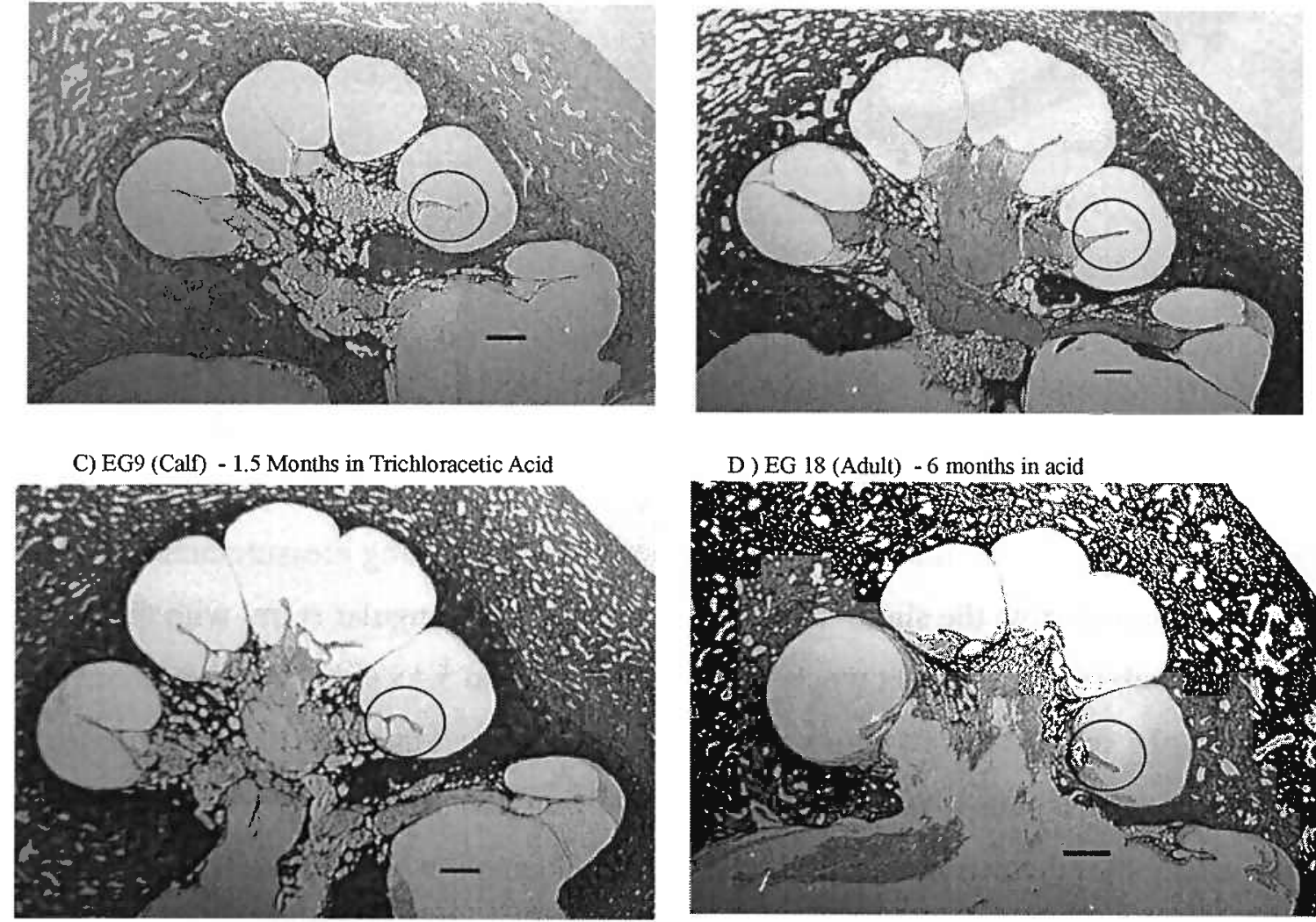

D) EG 18 (Adult) -6 months in acid

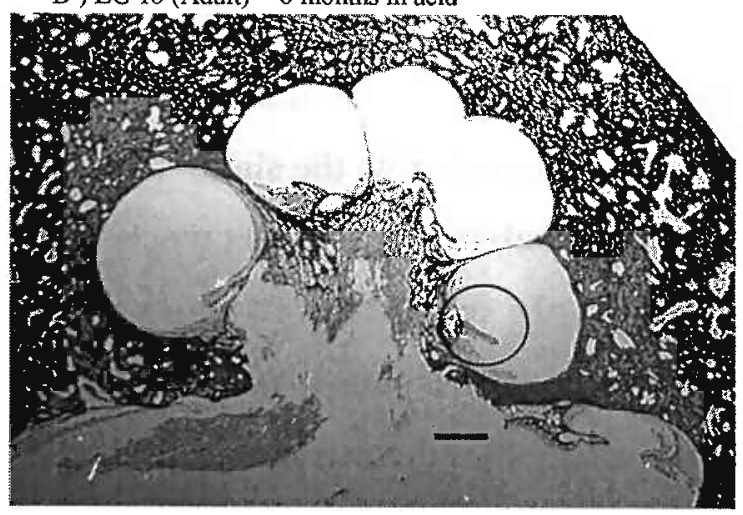

Figure 5. 13. Impact of acid decalcification on baleen whale ear bones. A) Ear from EG1 (Calf) decalcified in EDTA only. B) Ear from EG 4 (Calf), 2 months in trichloroacetic acid, 14 months in EDTA C) Ear from EG 9 (Calf), 1.5 months in trichloroacetic acid, 5 months EDTA D) Ear from EG 18 (Adult), 6 months in acid decalcification with bone wax in the oval and round windows to reduce the time the tissues of the inner cochlea were exposed to acid. The start of the second turn is circled in each slide for comparison of the acid effects on the inner osseous spiral lamina. The scale bar in each image represents $1 \mathrm{~mm}$. 
The histology slides were used primarily to measure the basilar membranes and to count ganglion cells for the specimens. Additional information is contained in these sections. EG 4 was preserved and sectioned with the ossicles intact. This specimen also showed signs of amniotic fluid remaining in the middle ear. This was the smallest specimen, smaller than a neonate that was believed to have died at birth.

a)

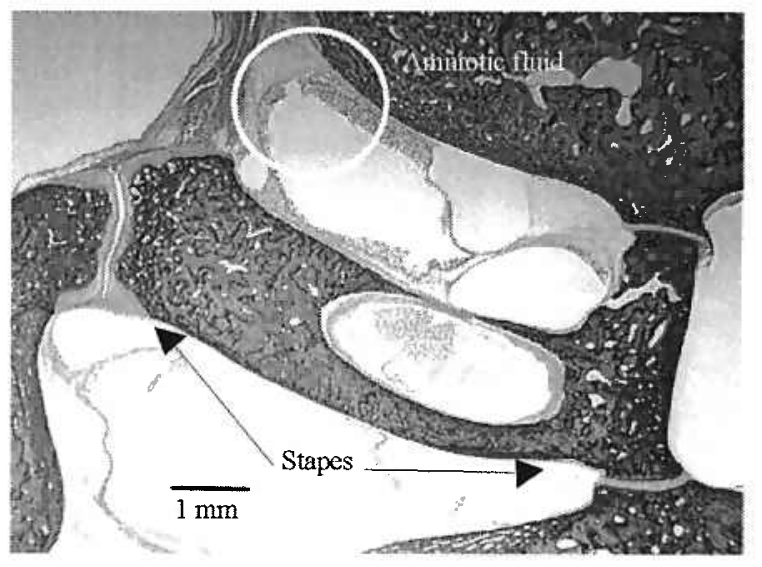

b)

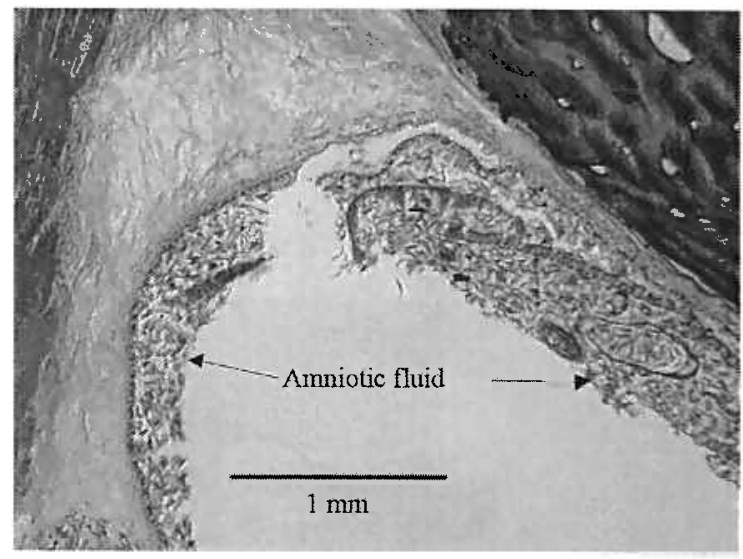

Figure 5.14. Insertion of the stapes into the oval widow and residual amniotic fluid in the middle ear cavity from EG 4.

\subsubsection{Basilar membrane measurements and ganglion cell counts}

Reconstructions of the basilar membranes were made using measurements of the basilar membranes from the slides and fitting them to an equiangular spiral with the same (a) value calculated for the right whale ears. Figures 5.8 and 5.15 show the measurements of intact basilar membranes fit onto a curve. The ganglion cell numbers at each slide are also included on the plots to indicate differences in ganglion cell numbers by position along the length of the membrane. The total corrected ganglion cell count for the two specimens with the best preservation are 37,930 for EG4 and 31,390 for EG9. This represents an extreme minimum number of cells as there is clear evidence of neuronal loss from disease and/or decomposition in these specimens. A count of 45,250 is obtained by combining the highest ganglion cell count for a particular position on the basilar 
membrane from EG 4 and EG 9. Calculation of the ganglion cell density/mm results in an average value of 1,842 ganglion cells $/ \mathrm{mm}$ (Table 5.5). Combined with the average cochlea length $(55.7 \mathrm{~mm}$, Table 5.4$)$, this results in a probable total ganglion cell number of approximately 102,500 ganglion cells. The direct counts are slightly greater than seen in human ears $(30,000)$ but much less than half of what has been reported for other baleen whales $(156,000)$ (Ketten, 2000). This lower count is likely the result of cellular loss from specimen decomposition. However, the calculated total ganglion cells based on the density/mm is comparable to the counts for both baleen whales and odontocete species (Ketten, 2000).

Table 5.5. Calculated ganglion cell density per mm of the basilar membrane. Ganglion cell densities were calculated using the ganglion cell count from a single $20 \mu \mathrm{m}$ slide at a percent position of the total membrane length. This value was then multiplied by 25 to get the density in $1 \mathrm{~mm}$ and correct for potential multiple counts of the same cell.

\begin{tabular}{c|ccc}
\hline $\begin{array}{c}\text { Percent membrane } \\
\text { length }\end{array}$ & $\begin{array}{c}\text { Density of ganglion } \\
\text { cells/mm for } E G 4\end{array}$ & $\begin{array}{c}\text { Density of ganglion } \\
\text { cells/mm for } E G 9\end{array}$ & $\begin{array}{c}\text { Highest ganglion cell } \\
\text { density }\end{array}$ \\
\hline 5 & 0 & 625 & 625 \\
10 & 900 & 650 & 900 \\
15 & 1525 & 850 & 1525 \\
20 & 3075 & 675 & 3075 \\
25 & 0 & 1225 & 1225 \\
40 & 2850 & 0 & 2850 \\
45 & 1600 & 1100 & 1600 \\
50 & 2875 & 1625 & 2875 \\
70 & 1575 & 0 & 1575 \\
75 & 2900 & 1325 & 2900 \\
90 & 1575 & 1675 & 1675 \\
98 & 1275 & 0 & 1275 \\
\hline Average density & 2015 & 1083 & $\mathbf{1 8 4 2}$ \\
\hline
\end{tabular}




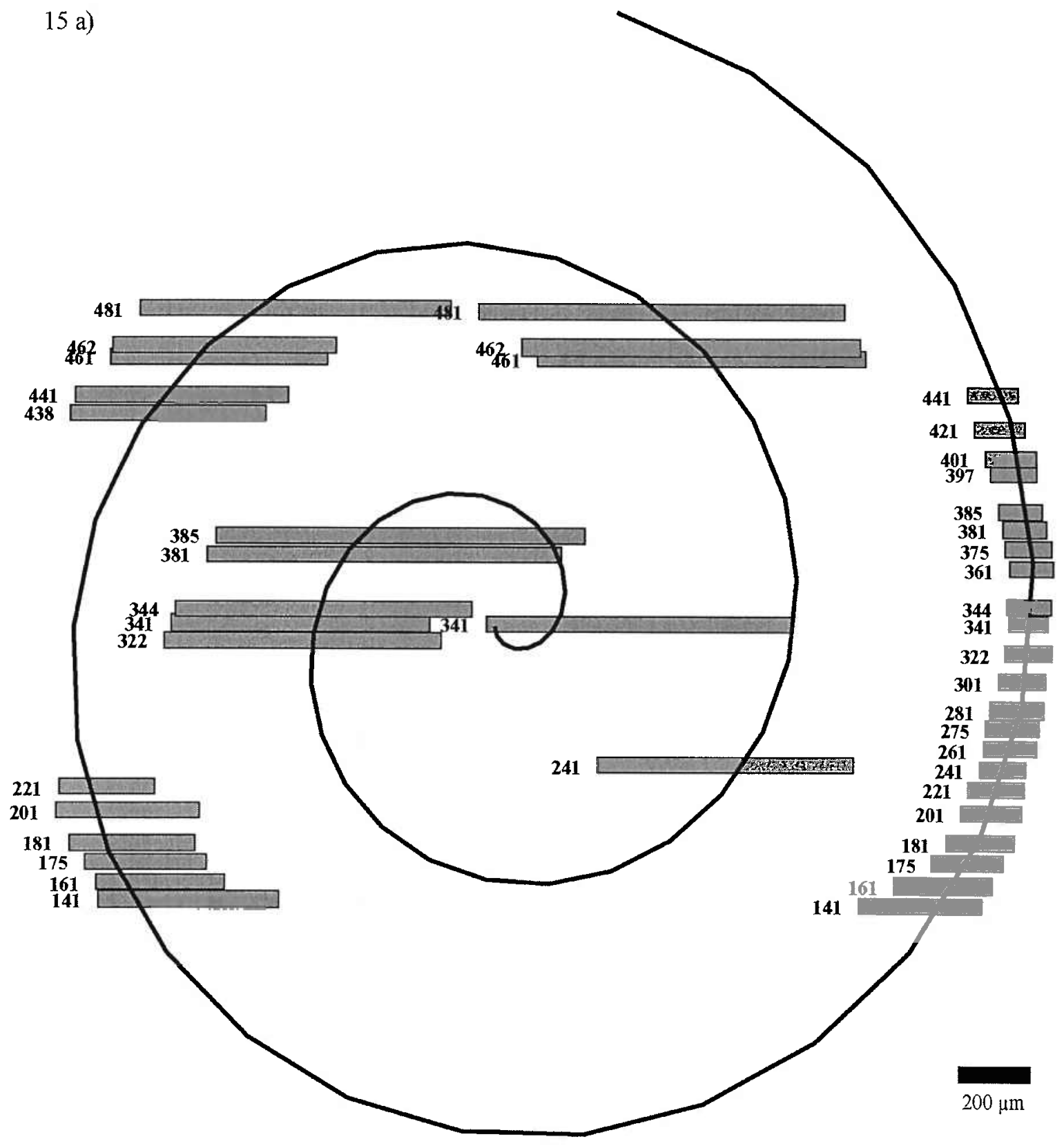


Chapter 5: Hearing

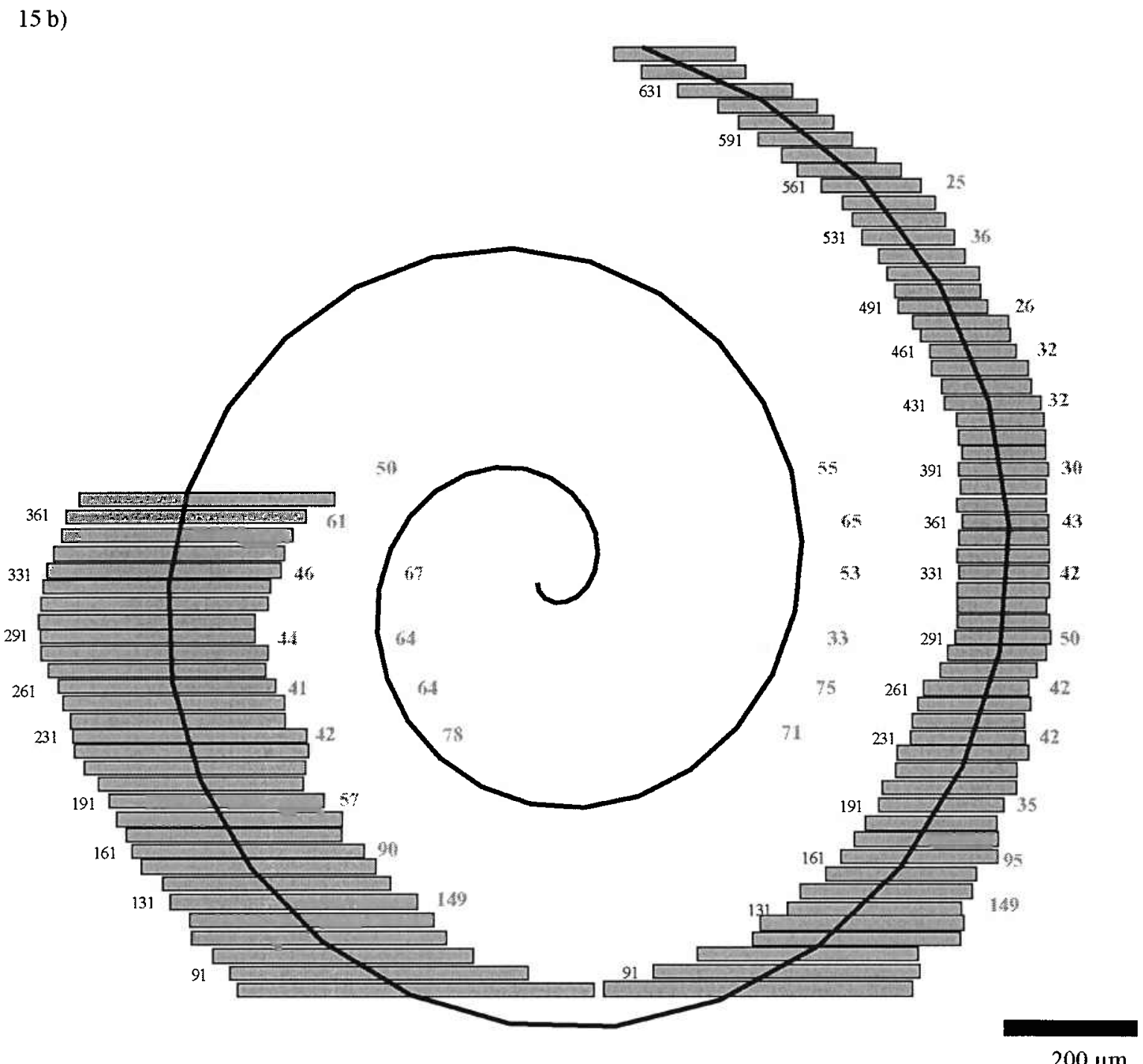




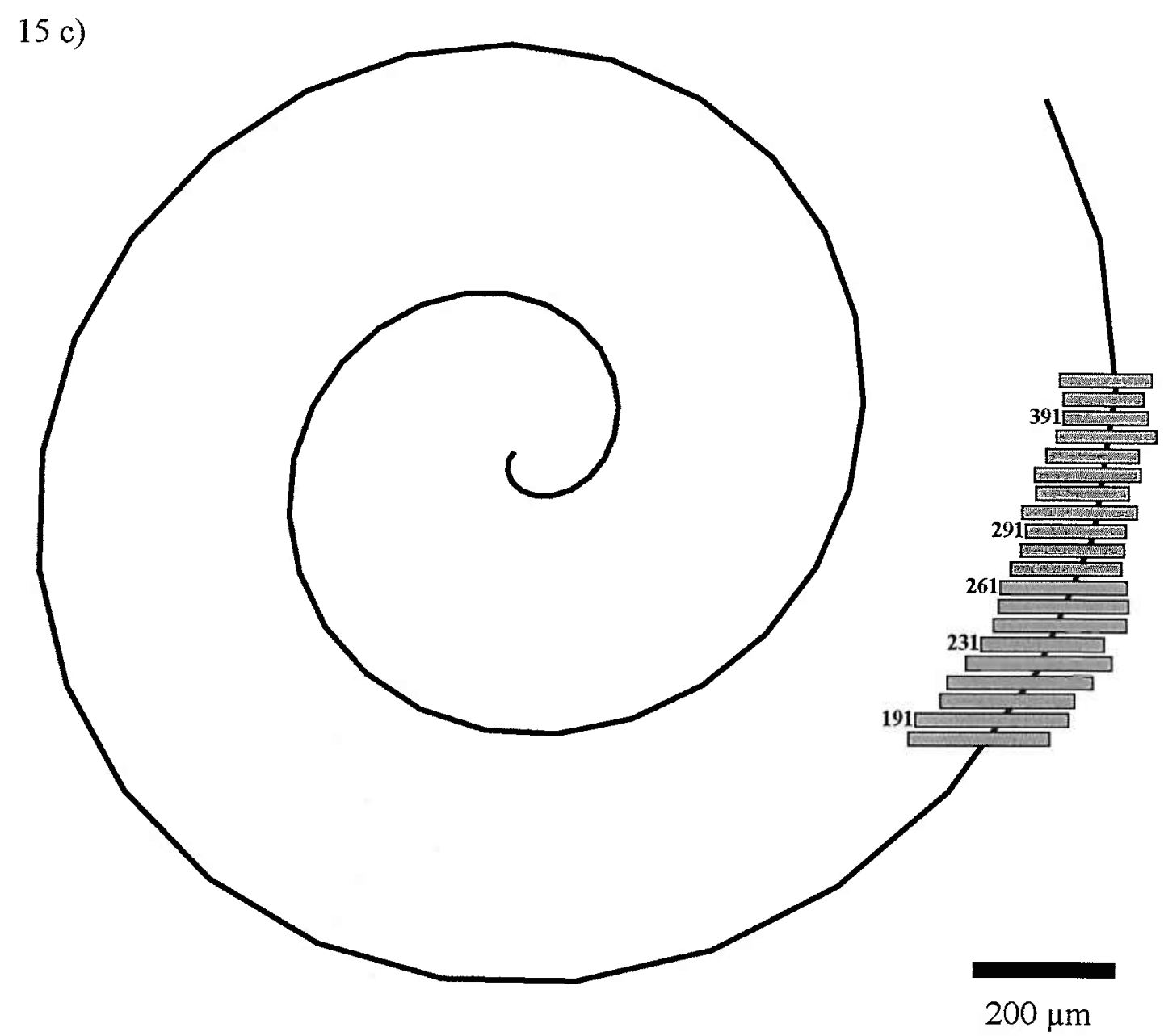

Figure 5.15. Reconstructions of basilar membranes and ganglion cell counts for each ear. A) EG 1 B) EG 9 C) EG 18. The basilar membrane width is represented by the gray bar. The section number is in black. The corresponding ganglion cell count, if any ganglion cells were present, is in gray. Useful ganglion cell counts were only possible for EG4 (Figure 5.8) and EG9.

\subsubsection{Frequency range for right whale hearing}

Table 5.6 shows the thickness/width ratios at different sections of the membrane length and the estimated frequency range of hearing for the right whale calculated from the data (D.R. Ketten, pers. comm.) using the model described in Ketten (1994). These numbers represent the functional range of the average of the ears measured in this study. These values also indicate a total possible hearing range of the right whale of approximately 10 $\mathrm{Hz}-22 \mathrm{kHz}$ (Ketten, 1994). 
Table 5.6. The thickness/width ratio of the basilar membrane measurements at different $\%$ of membrane length $($ apex $=0)$ from the 4 specimens as slides. The thickness/width ratio was only measured from nontangential sections. The predicted frequency range corresponding to these measurements is listed in $\mathrm{kHz}$ calculated from the model described in Ketten (1994).

\begin{tabular}{c|cc}
\hline $\begin{array}{c}\text { \% Membrane length } \\
\text { (apex to base) }\end{array}$ & Thickness/width ratio & $\begin{array}{c}\text { Predicted Frequency } \\
(\mathrm{kHz})\end{array}$ \\
\hline 2 & .0012 & .012 \\
20 & .0019 & .093 \\
50 & .0125 & 1.44 \\
80 & .0357 & 13.42 \\
83.5 & .0425 & 13.67 \\
90 & .05 & 18.28 \\
\hline
\end{tabular}

\subsection{Discussion}

This study represents a rare look at multiple ear specimens from a single baleen whale population. The long-term research efforts into this population provide additional data on the age of individuals that would be lacking for most other stranded baleen whale specimens. It is hoped that this study of multiple specimens will provide a more reliable description of the anatomy of right whale ears and, consequently, a more accurate estimate of the hearing of right whales based on this anatomy.

\subsubsection{Specimen collection and preservation}

North Atlantic right whale necropsies are exceptional in all respects. Numerous resources from federal and private organizations are used in recovery and necropsy for all observed fatalities. This is a result of the highly endangered status of this population. This has resulted in the collection of baleen whale ears that are in relatively good condition when fresh specimens are examined. Better specimens could be collected from fresh kills in populations of baleen whales where whaling still occurs, such as the bowhead and gray whale hunts in North America, or from minke, sei and fin whales from Norwegian, Icelandic or Japanese whaling fisheries. This is not an option for any right whale population anywhere in the world. 
This study indicates that collection of ear bones from right whales in any state of decomposition is worthwhile. This may have great application to collection of specimens from other right whale species for which resources and funding for research are much more limited. Ears collected from highly decomposed specimens often still retained the ossicular chain. Specimens with moderate decomposition often retained the tough glove finger and lining of the tympanic chamber. All of these specimens were useful for measurement of size, length and number of turns in the cochlea of the right whale. Decalcification and histological processing of two Code 3 (moderate decomposition) specimens (EG 4 and EG 9) resulted in very useful basilar membrane measurements and ganglion cell counts. Comparative specimens in any condition from other right whale populations would be useful to determine if there are any major differences in ear anatomy among the three proposed species of right whales.

\subsubsection{CT scanning results}

The CT scanning of specimens proved useful for a variety of in situ measurements. Both 2-D and 3-D images were useful in describing the right whale cochlea. The 2-D reconstructions were useful to evaluate the condition of the middle ear and to detect any remnants of the VIIIth nerve and possible basilar membrane retention in the ears assisting with selection for further dissection and histological processing. Cochlear length could be determined both from measurement of radii from cross-sections and from direct measurement from 3-D reconstructions of the canal. The total number of turns could easily be observed from the same 3-D reconstructions.

The position, orientation and relative size of the vestibular system could be determined from these scans. There was a problem with 3-D reconstruction of the semicircular canals in these specimens. The turns of the canals were often so thin, and the density of the bone surrounding them so similar to the rest of the bone in the area, that only partial reconstruction of the canals from the base was possible. Injection of a contrast medium may enhance the ability to image these canals. Histology indicates that 
the canals are complete and functional, with remaining ganglion cells found in some specimens.

The position and orientation of ossicles could be observed in situ, which will allow for later estimates of middle ear transfer functions for this species. Scans of entire temporal complexes were useful for measurement of the size of the tympanic and the periotic bones. Further, all of these observations were made without disturbing or destroying the specimen. These measurements can be compared to results from histology to determine the degree of shrinkage resulting from that process.

\subsubsection{Cochlear length measurements}

The use of CT 2-D cross sections to measure the radii of the cochleae in different positions was extremely sensitive to the orientation of the cross-section. The base of the right whale cochlea from the oval window opening is more ovoid than round and is complicated by the terminal hook of the cochlea near the vestibule. The selection of a cross-section perpendicular to this long axis of the base provided the most consistent projection for standardizing the measurements made on all specimens and yielded estimates that were within $5-10 \%$ for right and left ears from the same specimen. The actual angular position of the measurement had to be calculated based on the total number of turns for each ear.

\subsubsection{Cochlear length related to body size}

Cochlear length has been shown to correspond to body size in many species (Ketten, 1984; Echteler et al., 1994). Measurements of total body length versus cochlear length suggest that the same trend holds within the species for adults. The specimens in this study were from 7 calves and juveniles and 5 adults. There was a strong trend for larger cochlear size to be correlated with larger body size for the adults (Figure 5.9a). However, there was no apparent correlation between cochlear length and body size for 
calves (Figure 5.9b). This is expected because it is likely that the cochlea is near its maximum size at birth, while the body is expected to grow enormously over the first few years of life. Another potential problem with the correlation of cochlea size to body length in cetaceans is that it is not clear that maximum body length in adult baleen whales is predetermined, i.e., does a relatively large cochlea as a calf indicate a probable larger total body length at adulthood? Indeed, it is not known at what age baleen whales stop growing in length, with some indication that size may continue to increase for much of their life. The largest individuals in the North Atlantic right whale population are also thought to be the oldest known individuals in the population, such as a female, "Admiral" that was an adult at first sighting and has never been seen to calve in 23 years of sighting history.

\subsubsection{Gross dissection}

There were several aspects of the temporal bone anatomy seen in all dissected specimens that are worthy of note. First, the juncture between the periotic and tympanic bones is extremely stable, with $2-5 \mathrm{~mm}$ thickness of bone providing two points of connection. The largest contact point, directly lateral of the cochlea itself, was often 1-2 $\mathrm{cm}$ in length. The second connection, at the anterior end of the periotic was generally smaller and thinner. These two connections formed an arch between the periotic and tympanic through which the glove finger projected laterally.

A spongy layer of sharp spicules of bony and fatty tissue covered the dorsallateral side of the periotic bone. This spongy layer was very difficult to remove from the specimens, and it covered a dome of very dense bone in the periotic that projected laterally from the side of the cochlear duct itself. It was noted that this very dense layer of bone was one of the major roadblocks to decalcification in these specimens. This dense bone and spongy pad correspond to a position immediately between the bony flanges that wedge the ears against the skull. If baleen whales do hear primarily through bone conduction, the flanges may function to direct the sound to the dense bone surrounding 
the cochlea, with the spongy pad reducing incoming bone conduction of sound from other directions. Could baleen whales have bone equivalents of pinnae to selectively channel the sound? Alternatively baleen whales might not hear through bone conduction, and the spongy pad of bone against the very dense bone surrounding the cochlea could function to isolate the cochlea from vibrations of the skull.

The corpus cavernosum covered the interior of the tympanic bone in many specimens. This lining could function to regulate the pressure in the middle ear if regulated fluid flow provided expansion of this lining into the chamber. The malleus was supported by a bone strut that connected to the outer wall of the tympanic bone, close to the insertion of the glove finger. The strut can be seen clearly in Figure 5.3, projecting from the left side of the malleus and connecting to the wall of the tympanic bone. A very large stapedial muscle can be seen in slides from EG4, suggesting that there is substantial support of the ossicular chain both from muscles and bony supporting structures. The presence of a round window provides further argument for ossicular motion. If there were no movement of the oval window, why isn't the round window sealed? Whether these supports function to maintain the orientation of the ossicles under high pressure or are used to limit sound transduction into the inner ear, it seems likely that the ossicles are functional in this species. Even with the strut and the muscle present, the ossicular chain moves. The puzzle of potential bone conduction of sound directly to the inner ear and an apparently functional ossicular chain and highly vascularized middle ear cavity needs to be addressed in this species.

\subsubsection{Decalcification}

The duration of decalcification of right whales ears was longer than would be predicted from the sheer size of the ears. The density of the bone surrounding the cochlea is the major explanation for this. The duration of the decalcification of the periotic bone alone ranged from $6-16$ months, depending on the size of the specimen and the time in 
acid. Specimens decalcified solely in EDTA took roughly 20 months to decalcify and have yet to be processed into slides.

$5 \%$ trichloroacetic acid was used in an attempt to accelerate the rate of decalcification in the specimens presented here. The time in acid ranged from $1.5-6$ months. The acid did substantially speed of the rate of decalcification. For example, EG18's left ear decalcified in acid in 6 months, but EG 18's right ear took 20 months in EDTA. Acid also resulted in the loss of soft tissue within the cochlea. Figure 5.13 illustrates the effects that acid had on the specimens. One specimen, EG 18, had bone wax plugging both the oval and round window, but the wax dissolved quickly in the acid, exposing the inner ear to the acid. This specimen in particular had severe artifacts from the acid decalcification. Most of the soft tissue (ganglion cells, basilar membranes, spiral ligament) were completely dissolved by the acid, leaving only the most robust, thickest part of the basilar membrane for measurements. A conclusion from this study is that ears, including even large baleen whale ears, should not be decalcified with acid. A chelating agent such as EDTA is far superior. EDTA reduces artifacts from decalcification and results in better preservation of fragile membranes (Schuknecht, 1993). In baleen whales, the width and thinness of the basilar membrane toward the apical turns is important to preserve for accurate measurements to estimate low frequency hearing sensitivity. Unfortunately, membranes in that region are the most fragile and the first to be lost in acid decalcification. None of the specimens decalcified in acid retained any basilar membrane beyond the first turn of the cochlea. Microwave techniques may prove useful in baleen whale ear decalcification if an increase in decalcification rate is needed. However, this method has yet to be tested with baleen whale ears.

\subsubsection{Histology}

Preservation of baleen whale ears with the ossicles intact may add a great deal more information to studies of the middle ear function of these species. One specimen in this study was decalcified with the entire ossicular chain intact. This specimen showed 
both muscle attachment and connections between ossicles that are likely to be important for function in baleen whales.

Specimens from calves and adults made it possible to observe aging effects, such as demineralization of the periotic bone in older whales. Figure. 13 a-c shows ears from calves while (d) is an ear from an adult, $23+$ years of age. The latter has lower bone surrounding the cochlea. This change is not merely a result of differences in decalcification of the preserved specimen, but rather a real density difference that was evident from CT scans before the ear was dissected.

\subsubsection{Basilar membrane measurements and ganglion cell counts}

The basilar membrane of the right whale is consistent with previously described measurements of baleen whale basilar membranes. The base of the basilar membrane is thicker and narrower than the apical turn which is extremely thin and very wide. The measurement of the basilar membrane width was easily done using a 40x light microscope. Higher magnification, using a 100x oil immersion lens was necessary for measurements of membrane thickness, particular for thinner sections. The apical turn of the right whale ear has membranes that may be thinner than can be accurately measured by traditional light microscopy and are perhaps best examined by transmission electron microscopy.

The distribution of ganglion cells in the preserved specimens indicate that there may be variable ganglion distribution and possibly hair cells in different regions in the cochlea of right whales. However, the post-mortem decomposition of the specimens makes interpretation of the remaining ganglion cells difficult. Combining the counts of the best preserved sections of the basilar membranes from EG 4 and EG 9 still yielded a ganglion cell count that was significantly lower than any reported ganglion cell count for any cetacean species. The estimated ganglion cell densities/mm coupled with the average length of the right whale basilar membrane yielded a count comparable to those of other cetaceans. It is notable that the ganglion cell count of the right whale, presented here, and 
other baleen whales rival those of odontocetes (Ketten, 2000). These counts are much higher than any terrestrial mammal and may indicate that baleen whales also have a sophisticated auditory system (Ketten, 2000).

\subsubsection{Hearing range for the right whale}

The total hearing range for the right whale predicted from measurements made in this chapter is $10 \mathrm{~Hz}-22 \mathrm{kHz}$ with functional ranges probably being $15 \mathrm{~Hz}$ to $18 \mathrm{kHz}$ (D.R. Ketten, pers. comm.). These estimates were made using the model described in Ketten (1994). The apical measurements of the basilar membrane indicates better low frequency hearing than in humans while the capacity implied by the basal end of the membrane is slightly higher in frequency but similar to human ears. As expected this range corresponds well to the sounds produced by right whales (Chapter 3 ). Both this frequency range, and the frequency range of right whale sounds, overlap with many anthropogenic noise sources, suggesting that noise could potentially have a negative impact on hearing, localization and communication in this species.

\subsection{Conclusions}

This study provides a comprehensive description of a relatively large collection of baleen whale ears. Generally it is difficult to collect baleen whale ear specimens as large whale strandings are relatively rare in comparison to strandings of small odontocetes. As expected there was variation in the size, length, and number of turns of cochlea from different individuals but consistent intra-species form and length. Hearing ranges and vocalizations are consistent in frequency and total spectral distribution. 


\subsection{LITERATURE CITED}

$\mathrm{Au}$, W. W. L. 2000. Hearing in whales and dolphins: An oveview. In: Hearing by whales and dolphins (Ed. by Au, W. W. L., Popper, A. N. \& Fay, R. R.), pp. 1-42. New York: Springer-Verlag.

Clark, C. W. \& Clark, J. M. 1980. Sound playback experiments with southern right whales (Eubalaena australis). Science, 207, 663-665.

Cummings, W. C. \& Thompson, P. O. 1971. Gray whales, Eschrichtius robustus, avoid the underwater sounds of killer whales, Orcinus orca. Fishery Bulletin, 69, 525530 .

Echteler, S. W., Fay, R. R. \& Popper, A. N. 1994. Structure of the mammalian cochlea. In: Comparative hearing: Mammals (Ed. by Popper, A. N.), pp. 134-171. New York: Springer-Verlag.

Elder, J. H. 1934. Auditory acuity of the chimpanzee. J. Comp. and Physiol. Psychol, 17, 157-183.

Fay, R. R. 1992. Structure and function in sound discrimination among vertebrates. In: The evolutionary biology of hearing (Ed. by Popper, A. N.), pp. 229-267. New York: Springer-Verlag.

Fay, R. R. 1994. Comparative auditory research. In: Comparative hearing: Mammals (Ed. by Popper, A. N.), pp. 1-17. New York: Springer-Verlag.

Frankel, A. S., Joseph R. Mobley, Jr. , Louis M. Herman. 1995. Estimation of auditory response thresholds in humpback whales using biologically meaningful sounds. In: Sensory Systems of Aquatic Mammals (Ed. by Kastelein, R. A., Thomas, J. A. \& Nachtigall, P. E.), pp. 55-70. Woerden, The Netherlands: De Spil Publishers.

Greenwood, D. G. 1961. Critical bandwidth and the frequency coordinates of the basilar membrane. Journal of the Acoustical Society of America, 33, 1344-1356.

Greenwood, D. G. 1962. Approximate calculation of the dimensions of traveling-wave envelopes in four species. Journal of the Acoustical Society of America, 34, 13641384.

Greenwood, D. G. 1990. A cochlear frequency-position function for several species-29 years later. Journal of the Acoustical Society of America, 87, 2592-2605. 
Ketten, D. 1984. Correlations of morphology with frequency for Odontocete cochlea: Systematics and Topology. Ph.D. Thesis. The Johns Hopkins University, Baltimore.

Ketten, D. R. 1992. The marine mammal ear: Specializations for aquatic audition and echolocation. In: The evolutionary biology of hearing (Ed. by Webster, D., Fay, R. R. \& Popper, A. N.), pp. 717-754. New York: Springer-verlag.

Ketten, D. R. 1994. Functional analyses of whale ears: Adaptations for underwater hearing. I.E.E.E. Proceedings in Underwater Acoustics, 1, 264-270.

Ketten, D. R. 2000. Cetacean ears. In: Hearing by whales and dolphins (Ed. by Fay, R. R.), pp. 43-108. New York: Springer-Verlag.

Ketten, D. R., Skinner, M. W., Wang, G., Vannier, M. W., Gates, G. A. \& Neely, J. G. 1998. In vivo measures of cochlear length and insertion depth of nucleus cochlear implant electrode arrays. Annals of Otology, Rhinology \& Laryngology, 107, 116.

Ketten, D. R. \& Wartzok, D. 1990. Three-dimensional reconstructions of the dolphin ear. In: Sensory abilities of cetaceans (Ed. by Kastelein, R.), pp. 81-105. New York: Plenum Press.

Manley, G. A. 1971. Some aspects of the evolution of hearing in vertebrates. Nature, 230, 506-509.

Nadol, J. B. 1988. Quantification of human spiral ganglion cells by serial section reconstruction and segmental density estimates. Am. J. Otolarygol, 9, 47-51.

Parks, S. E. 2003. Response of North Atlantic right whales (Eubalaena glacialis) to playback of calls recorded from surface active groups in both the North and South Atlantic. Marine Mammal Science, 19, 563-580.

Payne, K. B., Langbauer Jr., W. J. \& Thomas, E. M. 1986. Infrasonic calls of the Asian elephant (Elephas maximus), Behavioral Ecology and Sociobiology., 18, 297-301.

Payne, R. S. \& Webb, D. 1971, Orientation by means of long range acoustic signalling in baleen whales. Annals of the New York Academy of Science, 188, 110-141.

Schuknecht, H. F. 1993. Pathology of the ear. Philadelphia: Lea and Febiger.

Tyack, P. 1983. Differential response of humpback whales, Megaptera novaeangliae, to playback of song or social sounds. Behavioral Ecology and Sociobiology, 13, 4955.

Urick, R. J. 1983. Principles of underwater sound. Los Altos, CA: Peninsula Publishing. 
von Békésy, G. 1960. Experiments in hearing. New York: McGraw-Hill Book Co.

Watkins, W. A. 1981. Activities and underwater sounds of fin whales. Scientific Report to the Whales Research Institute, 33, 83-117.

Watkins, W. A. 1986. Whale reactions to human activities in Cape Cod waters. Marine Mammal Science, 2, 251-262.

West, C. D. 1985. The relationship of the spiral turns of the cochlea and the length of the basilar membrane to the range of audible frequencies in ground dwelling mammals. Journal of the Acoustical Society of America, 77, 1091-1101.

Yost, W. A. 2000. Fundamentals of Hearing: An introduction. San Diego: Academic Press. 
Chapter 5: Hearing 


\section{CHAPTER 6. SUMMARY AND CONCLUSIONS}

\subsection{SUMMARY}

The goal of this thesis was to describe how the North Atlantic right whale uses sound for communication. The surface active group (SAG) was selected as a focus because it was the most commonly observed social interaction known to be associated with sound production. This social interaction made it possible to study the signaler, the signal, and the receiver in the communication chain. The analysis of this interaction included a description of the occurrence and composition of the SAGs (Chapter 2), summary of the sound production by right whales in SAGs (Chapter 3), the response of whales to playback of whale sounds recorded from SAGs (Chapter 4), and anatomical modeling to estimate the frequency range of right whale hearing that should related to perceptions of sounds produced in SAGs (Chapter 5). All of these pieces can be combined to look at how sound is used by right whales to form and maintain these groups. This chapter will attempt to summarize how the results of the previous five chapters address the initial hypotheses set out at the start of the thesis.

\subsubsection{Chapter 1: Social interactions in baleen whales}

The goal of the first chapter was to provide a literature review of evidence for social behavior in baleen whales. Most studies demonstrating complex long-term social bonds in cetaceans have focused on odontocetes. Previous research has suggested that long-term social bonds are rare and relatively short-lived in baleen whales compared to odontocetes. However, baleen whales have large migratory ranges and are capable of acoustic communication over long distances. Research investigating long-term bonds in baleen whales needs to be expanded in scope to include the possibility of social interactions over many years and acoustically mediated social interactions between individuals several miles apart that would not be apparent from visual observations alone. 
This review indicates that social behaviors, particularly long-term bonds between individuals, are potentially an important aspect of baleen whale biology. Short-term social interactions have been documented in several baleen whale species relating to feeding or mating. The feeding groups of humpback whales in Alaska show that baleen whales can maintain relationships with individuals over many years. In most baleen whale species, the mating season is the most social time of the year, and presumed mating behavior seems to predominate social interactions. Production of specific sounds has been documented in specific baleen whale social interactions, both for feeding and mating. These basic observations indicate that further research into social bonds in baleen whales is necessary, including consideration of the importance of acoustic social communication.

\subsubsection{Chapter 2: Social behavior of North Atlantic right whales (Eubalaena glacialis) in surface active groups.}

Chapter 2 addresses the question of the function of North Atlantic right whale SAGs. The frequent observation of sexual activity coupled with a typical group composition of one or more females with multiple males have led many to speculate that these groups are reproductive in function. The prevalence, duration and activity levels of these groups further suggest that participation is a high priority for right whales, supporting the hypothesis that they may function for reproductive purposes. Male right whale anatomy suggests that right whales have a promiscuous mating system (Brownell \& Ralls, 1986). Right whales frequently engage in sexual activity with multiple males achieving intromission with a single female in one SAG. In the absence of any other information these groups have been assumed to function for reproduction in this species (and some SAG very well may). The data presented in Chapter 2 provide an analysis of sightings of North Atlantic right whales in SAGs from 1992-2001. There were five main predictions tested in Chapter 2. 
1. If all SAGs are reproductive, the number of SAGs observed through out the year will show a seasonal peak during the breeding season.

2. The group composition of SAGs will be consistent with a reproductive function.

3. If SAGs involve fertilization, the number of SAGs observed will be correlated to the number of calves born 11-13 months later.

4. Males will be sighted in SAGs more frequently than females.

5. Group composition of SAGs will be different at different times of the year, with reproductive groups more common during the breeding season.

1. If all SAGs are reproductive, the number of SAGs observed through out the year will show a seasonal peak during the breeding season.

SAGs are commonly observed on all right whale surveys in all regions during all but one month of the year in the North Atlantic. My summary of SAG observations indicates that there may be an increase in SAG activity in the late summer and early fall. This trend was seen in all ten years, even though the total number of SAGs sighted was highly variable. The number of all-male groups increases in the late summer, and this may indicate an increased interest on the part of males to engage in mating behavior. This may be related to an increase in testosterone that may signal males preparing for the breeding season. Other baleen whales have shown seasonal trends in testis size from whaling data (Berta \& Sumich, 1999).

2. The group composition of SAGs will be consistent with a reproductive function.

The results presented in Chapter 2 summarizing the sighting history of SAGs from the past ten years indicate that group composition is not always compatible with SAGs being purely reproductive in function. Reproductive groups would be expected to be composed, at least in part, of potentially reproductive animals in some combination (one or more potentially reproductive females with one or more potentially reproductive males). When the identity of individuals within the groups is examined, a number of different group compositions result that are not consistent with a reproductive function. 
These include groups in which the only female is pregnant, groups of only males, groups of only females, groups with a mother and her calf as the focal animals, or groups with a calf as the focal animal by itself. These groups cannot lead directly to fertilization. Either the definition of SAGs needs to be modified, or additional functions of these groups need to be considered.

What humans label as SAGs from surface observations may actually encompass a number of distinct social interactions from the right whale perspective. It seems naive to suggest that right whales do not know the sex and reproductive status of other individuals in the group. The costs of energy and potential injury in these SAGs are likely offset by some benefit other than fertilization. These benefits may include female assessment of males for later mate choice, practice improving later reproductive success, groups strengthening bonds between individuals for either feeding or reproductive purposes, or familial bonding to avoid incest later during mating.

Most SAGs appear to be a form of social interaction either directly or indirectly related to reproduction and fertilization. Any groups involving both sexually mature males and females fit into this category (Table 2.1). Many SAG compositions can be explained in terms of direct reproduction or practice of these behaviors. SAGs with multiple females often involve one mature and one immature female. Females have their own set of behaviors when involved in SAGs. Females appear to be adept at getting out of SAGs. Rarely do females in SAGs look harassed or stressed by the situation. Observations of females deftly evading all males present and completely disappearing are common and the female's departure generally indicates the end of the SAG. The immature female may need to learn particular behaviors in SAGs from the older, more experienced, female. During two observations of SAGs where a second, younger female joined a SAG already in progress, the older female departed the group within 20 minutes. It is worth investigating whether females in multiple-female groups are closely related because it would improve the transmission of a female's genes to help improve the reproductive success of close kin. 
Non-reproductive group compositions (Table 2.1) can be indirectly related to reproduction. SAGs involving calves may be important in the early development of right whale social interactions. The mother is often in the group with the calf, suggesting that involvement of the calf in the SAG is intentional. The mother may involve the calf in a SAG for it to experience the behaviors involved in these groups. These behaviors may include sound production and interactions with other individuals. However, calf involvement in SAGs occasionally appears to be less than voluntary. Some large males appear to keep the calf captive, occasionally even lifting calves out of the water on their bellies, possibly to ensure the return of the mother. Older calves may effectively learn how to avoid this coercion.

SAG involvement may represent practice of behaviors important for later SAG participation. Many of the all-male groups are composed primarily of immature males and these groups are more frequently observed in the fall before the presumed breeding season. Males may be practicing SAG behaviors or establishing some sort of dominance relationships before females become receptive (Kraus \& Hatch, 2001). The all-female groups, most commonly seen on the calving grounds, may indicate female interest in or attendance on pregnant females prior to calving. These groups bear further investigation, possibly in the Southern right whale population where the number of females is larger.

The lack of obvious differences in behavior of individuals in groups with pregnant vs. potentially receptive females is particularly puzzling. Attempts on my part to rationalize this behavior in terms of direct benefit to the female have failed. The female is losing time from feeding and is exposing herself to infection or injury from close association and intromission with males. In fact, this behavior seems to be maladaptive for right whales, and yet it is not uncommon, with $40 \%$ of pregnant females being observed in at least one of these groups. The high hormonal levels of pregnant female right whales may play a role in motivating this behavior (Rolland et al., 2002). Other mammalian species show evidence of increased sexual behavior in females early in pregnancy that has been explained by fluctuations in hormonal levels. Further research on hormone levels in female right whales during pregnancy may shed light on this as early 
results indicate very high levels of reproductive hormones in the pregnant females (Rolland et al., 2002).

3. If SAGs involve fertilization, the number of SAGs observed will be correlated to the number of calves born 11-13 months later.

The duration of gestation in Southern right whales was estimated using the lengths of neonates (Best, 1994). Neonate data came from historic whaling records and stranding data along the South African coast (Best, 1994). The gestation period in the South Atlantic right whale is estimated to be 11-13 months (Best, 1994). This gestation period is based on the assumption of linear growth through most of the developmental stage, using 24 August as the mean date of birth (Best, 1994). This gestation estimate does not take into account the time for implantation of the embryo, the possibility of delayed implantation, nor does it address issues about possible periods of non-linear growth at any stage during gestation.

An obvious problem with the suggestion that all mating in SAGs leads to fertilization comes from the discrepancy between year round SAG observations and the seasonal calving. It has been assumed that the gestation period in the North Atlantic right whale should be the same as the South Atlantic right whale. Calving in the North Atlantic right whale is highly seasonal, ranging from early December through February. This would mean that productive mating should take place sometime between November and March. SAGs are seen in all parts of the known range of North Atlantic right whales and have been sighted in 11 out of 12 months of the year. This observation alone indicates that not all right whale SAGs can result in fertilization.

Unfortunately, the distribution of North Atlantic right whales during the assumed breeding season from November-March is relatively poorly understood. Calving females or other adults are sighted on the calving grounds and variable numbers of individuals feed in Cape Cod Bay during this period. The remainder of the population is effectively "missing" during the period of presumed fertilization, resulting in a lack of observations during the right whale breeding season. Potential reproductive explanations for the 
observed pattern of SAG occurrence vary and include possible delayed implantation of embryos or synchronized seasonal cryptic ovulation by females with year round sexual activity resulting in synchronized calving events.

4. Males will be sighted in SAGs more frequently than females.

Right whales have a promiscuous mating system and male right whale anatomy is consistent with sperm competition (Brownell \& Ralls, 1986). Therefore, multiple males would be expected to mate with a single female. The data in Chapter 2 support this, as the composition of SAGs often leads to more males being present in each group. This trend is reflected in the individual sighting histories, where on average, males are seen twice as often in SAGs than females. This results is not particularly surprising and may be a result of higher motivation and lower costs on the part of males to engage in the observed social behavior.

5. Group composition of SAGs will be different at different times of the year, with reproductive groups more common during the breeding season.

There seems to be variation in composition of SAGs throughout the year. The potentially reproductive groups seem to be common, by percentage throughout the year. However, the total number of SAGs, and hence the total number of potentially reproductive SAGs, observed increases substantially in the late summer and early fall. This observation is consistent with an increase of SAG activity around the period of presumed breeding season. The non-reproductive groups show more seasonality in their occurrence. In particular, all-male groups and groups with calves increase in the summer months. Groups with pregnant females are most common on the calving ground, just prior to the female giving birth.

This thesis indicates that overly general definitions of social interactions can be misleading. Groups lumped together under the SAG label have distinctly different individual compositions and particular types of vocalizations are associated with different compositions. Right whales, at least in the Bay of Fundy, may be able to acoustically 
distinguish these groups. This would allow whales to choose which groups to join, resulting in the different compositions observed. The observation of similar behaviors in other coastal baleen whale species suggests that there may be similar mislabeling of social behaviors as reproduction. This point has already been addressed by Norris (1983) who labeled groups in gray whales as "wuzzles" to encompass several different functions.

While this thesis cannot answer all the puzzling aspects of SAGs, it does indicate that the groups labeled as SAGs likely represent multiple different interactions to right whales. Future studies, including detailed behavioral observations and the use of genetic relatedness data may be able to distinguish between these various hypotheses of SAG function.

\subsubsection{Chapter 3: Acoustic activity of North Atlantic right whales (Eubalaena glacialis) in surface active groups}

This chapter addresses the sound production by right whales in SAGs. It is likely that approaching whales use the sounds produced in these groups to locate them. Indirect evidence suggests that the Scream calls, the major type of sound produced by right whales in SAGs, are produced by the focal female to attract males, thereby increasing the number of males competing for access to the female. The evidence for this includes: on all recordings of SAGs in which only a single female was present, single Screams were only heard alone and never simultaneously, no instances of Screams being made while the focal female whale was breathing, and the cessation of Scream production with the departure of the focal female (Kraus \& Hatch, 2001). Additional sound types are produced in SAGs. This chapter describes all sounds produced in SAGs and addresses sound production of different individuals within the group. There were nine predictions presented for Chapter 3 at the beginning of the thesis. Here I present 10 predictions that resulted from the work in Chapter 3. 
1. Particular types of sounds will be produced in SAGs.

2. The focal animal produces the Screams in SAGs.

3. Individual variation in Scream calls allow for individual recognition.

4. Males produce the Gunshot sounds in SAGs.

5. The focal animal does not produce gunshot sounds in SAGs.

6. Calves produce the Warble sounds in SAGs.

7. Males produce the Upcalls in SAGs.

8. The number of gunshots produced in a SAG will be correlated to the number of males present in the group.

9. Sound production will vary with group composition.

10. Source level for right whale sounds will vary with ambient noise levels.

1. Particular types of sounds will be produced in SAGs.

Several different types of sounds are produced by right whales in SAGs. These include Screams, Gunshots, Noisy Blows, Upcalls, Warbles and Downcalls. The Scream, Gunshot, and Noisy Blows were the most common sounds produced.

2. The focal animal produces the Screams in SAGs.

The Screams appear to be produced by the females in the groups. No Screams were recorded when the focal female was breathing. The only overlapping Scream calls were recorded in groups with multiple females present. The call rate of Scream production does not increase with increasing number of males in the group. The SAGs often break up temporarily when the female goes on a dive. These periods correspond to periods of silence between bouts of sound production. The departure of the focal female always coincided with the end of Scream call production. These Scream calls appear to function to attract other whales to the group and possibly to maintain group cohesion. Females produce these Screams in bouts. 
3. Individual variation in Scream calls allow for individual recognition.

The variability of the Scream call types led me to hypothesize that individual recognition might be possible based on Screams. The initial results presented in Chapter 3 indicate that there may be variation for calls produced by individuals with a significant difference between the mean values for calls between different individuals. However, the methods used in this thesis were not adequate to confidently discriminate between individuals based on a small sample of Scream calls. More repeat recordings from the same individual will be needed to fully address this question.

$4 \& 5$. Males produce the Gunshot sounds in SAGs \& The focal animal does not produce gunshot sounds in SAGs.

I hypothesized that males produce Gunshot sounds in the SAGs. Assignment of Gunshot sound production to a particular individual is still difficult, but the rate of Gunshots is higher in groups with more males present and the Gunshot is the predominant sound documented in suspected all -male groups. Observation of lone males making stereotyped displays accompanied by Gunshot sound production further supports the idea that males produce the sounds (Appendix 2). Additional observations from the video recordings indicate that Gunshots are occasionally produced while the female is breathing, indicating that the males do produce some, if not all, of the Gunshot sounds recorded in these groups. Earlier description of Gunshot sound production indicated that this sound might be linked to agonistic or threatening interactions between individual whales (Clark 1983). This may be the case in SAGs where males are competing for access to the focal female. Gunshot sounds could also function as male advertisement signals within SAGs themselves. Given the behavioral settings in which Gunshots are produced, SAGs and lone male displays, it seems unlikely that these sounds function for echolocation. Similar impulse sounds in odontocete species have been predicted to have an echolocative function. It is likely that right whales can hear the echo returns from their Gunshots since they are clearly audible from the recording made in this thesis. Whether 
whales use the echoes is unclear, particularly given the behavioral contexts in which they are produced.

6.Calves produce the Warble sounds in SAGs.

I predicted that Warble calls were produced by calves, possibly only by female calves. They are only recorded in groups with calves and array-bearing data are consistent with this hypothesis. These Warble sounds were only recorded on two occasions. In one case the calf was female. The sex of the other calf recorded producing Warbles is currently unknown. Analysis of other recordings from SAGs with calves present did not detect any Warble sounds, and in several instances, the calves in these groups were known to be male. Do calves produce these calls to attract their mother's attention or do they represent an attempt on the part of the calf to mimic the Scream sounds produced by the mother? Perhaps these Warble sounds are early attempts by female calves to produce Scream calls in SAGs. When males begin to produce Gunshot sounds is unknown, but male calf involvement in SAGs may familiarize the calves with this sound.

\section{Males produce the Upcalls in SAGs.}

Evidence supports the hypothesis that males produce the Upcalls in SAGs. Females may also produce Upcalls in SAGs but the bearing data from the array only confirm males producing Upcalls while joining the group or the female is on a dive or departed the group. The production of Upcalls is commonly accompanied by what appears to be searching behavior of the male swimming back and forth across the area that the SAG was in before the female went on a dive. The observation of Upcalls being

produced by a male as he swam into a SAG for the first time suggests that these calls may be used to announce the male's presence. Similarly, the production of Upcalls when the female is essentially "missing" may indicate the male's presence to the female if she chooses to rejoin him and/or reform the SAG. 
8. The number of Gunshots produced in a SAG will be correlated to the number of males present in the group.

The Gunshot sound rate does appear to be related to the size of the group, although the correlation was only 0.5 . Since males seem to produce the Gunshot sounds and more males are present in larger SAGs, this result is not surprising. The call rate for the Scream call does not appear to be correlated with the group size. This is consistent with the prediction that the female produces these calls, as the number of females is typically lower than the number of males in a SAG.

9. Sound production will vary with group composition.

In general, most sound types were produced in all SAGs, regardless of composition. The proportion of sound types did vary based on the composition of the groups. Warble calls were only recorded when calves were present in the SAG. Screams were rarely heard in groups of all-male composition.

10. Source level for right whale sounds will vary with ambient noise levels.

North Atlantic right whales are commonly exposed to noise in the environment. Results from this thesis indicate that right whales may modify their sound production in response to noise in the environment. While there was no evidence for the broadband source level to increase in response to noise, there was evidence that right whale may shift more acoustic energy to the higher frequency components of the calls. This may increase the detection range for the signals in an environment where the majority of noise sources are from vessel engines with most energy in lower frequencies. The calculations of source level in right whale calls (Chapter 3) indicate that increasing ambient noise, particularly vessel noise, will reduce the range at which right whales can detect these calls. If the effective range of detection is important, this effect of masking may have serious impacts on this interaction, regardless of the ultimate function of the groups. 
The description of acoustic activity in the groups adds to the picture of SAGs as complex interactions between individuals, rather than simple groups with only one whale producing all the sounds to attract other whales to the group.

\subsubsection{Chapter 4: Playback experiments to North Atlantic right whales in the Bay of Fundy}

Chapter 4 addressed the issue of how right whales respond to the sounds produce in SAGs. The initial hypothesis underlying these playbacks is that right whales use the sounds produced in SAGs to find and join the groups. Playbacks of sounds recorded from SAGs to North Atlantic right whales make it possible to confirm that the sounds in the groups attract other whales. It is possible that males locate these groups by detecting a variety of sounds associated with SAGs, such as Screams, Gunshot sounds, Upcalls and loud, audible underwater blow sounds. The purpose of this experiment was to determine if the Scream calls produced by females in SAGs are sufficient to attract males to these groups without additional acoustic cues. To test if this is the case, playback trials using only Screams from SAGs or only Gunshot sounds were conducted. Gunshot sounds are produced both in SAGs and by lone animals, so the Gunshot playbacks were designed to determine if right whales would approach any sound associated with a SAG, even one that is not a reliable indicator of a SAG. If the Scream calls were the primary cue used by adult males to detect a SAG, few or no animals would be expected to approach the Gunshot sound playbacks. The playback experiments confirm that sound production in SAGs is important for other whales to find the groups. Five predictions were presented in this chapter.

1. Playback of Screams from SAGs will attract right whales.

2. Playback of Gunshot sounds from SAGs will not attract right whales.

3. Scream calls from a $S A G$ will attract significantly more males than females.

4. Playbacks of SAG sounds will attract more adults than juveniles. 
5. Gunshot sounds may stimulate approach and challenging from mature males by the production of more gunshots.

1. Playback of Screams from SAGs will attract right whales.

Playbacks of Screams from both North Atlantic and Southern right whales attracted right whales to the playback vessel. Not all whales exposed to the playbacks approached.

2. Playback of Gunshot sounds from SAGs will not attract right whales.

Whales do not appear to approach all sounds that indicate that a right whale is present (i.e. the Gunshot playbacks), but clearly preferentially approach Scream calls. The Gunshot sound playbacks did not attract any right whales.

3. Scream calls from a SAG will attract significantly more males than females.

Adult males were the predominant age/sex class that approached the North Atlantic right whale SAG playbacks. Both males and females, juveniles and adults, approached the playbacks of South Atlantic right whale Scream calls. This difference in response to the different Scream stimuli was unexpected. There are three potential explanations for the observed difference in response. The first possibility is that the Scream calls of these two species differ enough so as not to be recognized by North Atlantic right whales as right whale calls. Secondly, if right whales recognize the Scream calls of specific individuals, then the Scream calls from the SARW-SAGs were from individual whales that were completely unknown to the North Atlantic right whale population. In both cases, the novelty of these stimuli might attract attention from any member of the North Atlantic right whale population. A third potential explanation for the difference is that these recordings were made from the calving grounds of a SARW population which has a different demographic structure and behavioral context than that seen on the feeding grounds in the North Atlantic. 
4. Playbacks of SAG sounds will attract more adults than juveniles.

This prediction was initially made under the assumption that SAGs functioned for reproduction and would not typically involve sexually immature whales. Although Chapter 2 demonstrated that juveniles are commonly involved in SAGs, very few juveniles approached the North Atlantic right whale SAG playbacks. However, several juveniles and calves approached the South Atlantic right whale SAG playbacks. This difference may be a result of the SAG recordings presented representing two different types of SAGs (i.e. potentially reproductive from the North Atlantic right whales and non-reproductive from the Southern right whales).

5. Gunshot sounds may stimulate approach and challenging from mature males by the production of more gunshots.

Based on the observation of multiple males producing Gunshot sounds in SAGs suggested that these sounds might serve an agonistic function. It was unknown whether males would respond aggressively to the playback of these stimuli. The Gunshot sound playbacks elicited no approach response from any whale. No males approached or were recorded making Gunshot sounds in response to the playback.

The results from the playback experiments further confirm the idea that males produce Upcalls as they enter the SAGs. The only sounds recorded in the presence of whales approaching the playbacks were Upcalls. Several whales also showed evidence of searching behaviors when the playback transmission ended. Some whales were able to locate the source of the playback sounds up to 20 minutes after the end of the playback trial. Whether they were investigating the playback vessel itself, or remembered the precise location of the sound source they had been heading toward, remains unclear. 


\subsubsection{Chapter 5: Hearing in the North Atlantic right whale: Anatomical predictions}

The goal of this chapter was to make measurements of the right whale basilar membrane to use in published models to estimate the frequency range of hearing. There was one main prediction for this chapter.

The range of hearing sensitivities in the right whale will correspond to the range of frequencies produced in right whale vocalizations.

The results from anatomical modeling of the hearing curve of North Atlantic right whales indicate functional hearing between $10 \mathrm{~Hz}$ to $22 \mathrm{kHz}$. This frequency range of hearing corresponds well with the frequency range of the sounds that they produce (Table 3.3). There is complete overlap with the peak energy of vessel noise in the habitat and the frequency range of hearing in these whales. The North Atlantic right whales live in areas with relatively high levels of noise from shipping (Desharnais et al., 2000). It is conceivable that these elevated levels of noise could contribute to hearing loss of some frequencies over long periods of time unless the whales have adaptations for hearing that protect the inner ear. At the very least, the higher noise levels will reduce the range over which right whale can communicate.

\subsection{ADDitional CONSIDERATIONS}

The population studied for this thesis does not represent a healthy, stable baleen whale population. Rather, the North Atlantic right whale population is very small and currently under several stresses from anthropogenic and environmental factors. The most obvious limitations to conducting a study of "normal" behavior in any coastal baleen whale species are the reduced population sizes resulting from hundreds of years of whaling. All coastal baleen whale populations were reduced to very low numbers with some local populations being eliminated entirely. Importantly, most species of coastal baleen whales, including bowhead and gray whales, show behaviors that appear to be 
similar to those observed in the North Atlantic right whale. The SAGs and wuzzles of these two species appear to occur in both the breeding and feeding season, involving multiple individuals and sexual activity, even outside of the breeding season when mating for fertilization is thought to take place (Everitt \& Krogman, 1979; Norris et al., 1983; Swartz, 1986; Würsig \& Clark, 1993; Richardson et al., 1995).

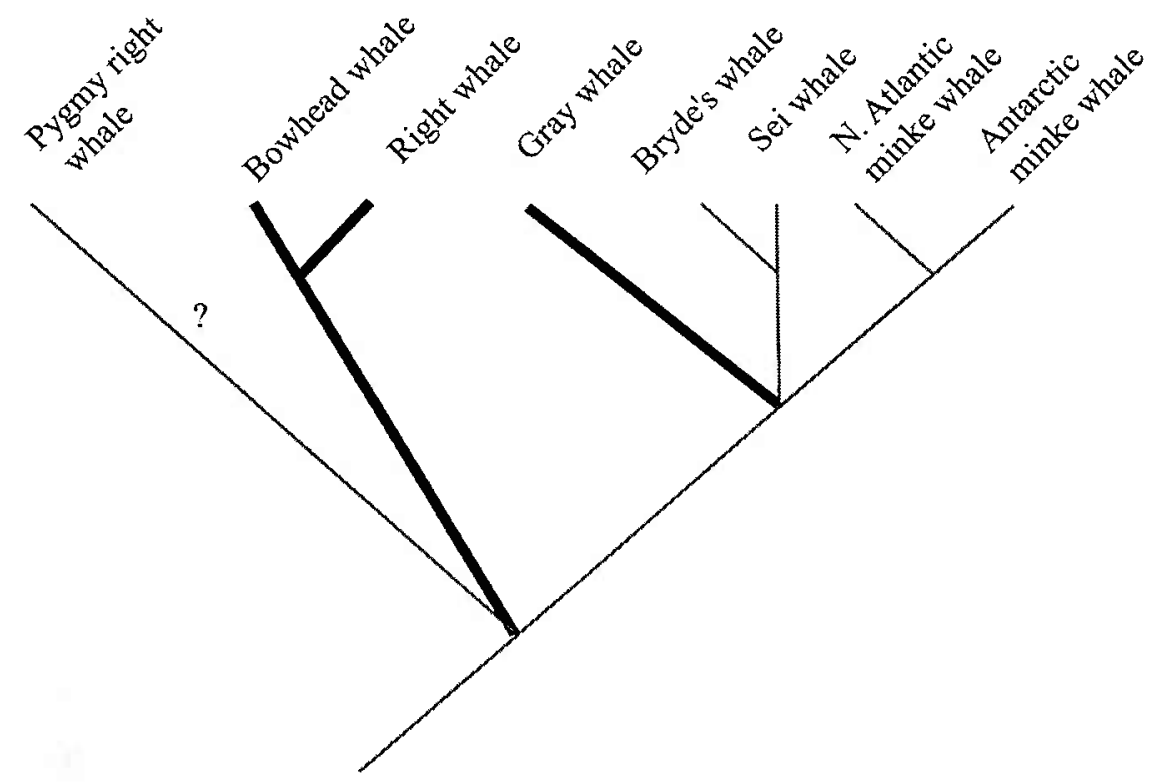

Figure 6.1. Relationship among mysticete species based on total evidence molecular data (Hasegawa et al., 1997). The darker branches indicate species with documented observations of SAG-like behaviors.

The question of how "normal" the behaviors seen in the North Atlantic right whales are is difficult to answer. However, the observation that similar behavioral interactions and sound production has been documented in several populations and species of coastal baleen whales strongly suggests that the behaviors seen in SAGs likely did not arise as a response to external stress or population bottlenecks, but rather have root in a historical ancestor common to all right whale species (Figure 6.1). Therefore, it is likely that the behaviors observed in this study are a result of long-term evolutionary changes in the species, and not a short-term abnormal response to severe stress and small population size. 


\subsection{Future RESEARCH NeEDS}

Several lines of future research are suggested from the results of this thesis. First and foremost, the playback experiments that were intended to be a straightforward experiment to document right whale response to SAG sounds, created many more questions than they answered. Unintentionally, these experiments ended up demonstrating the ability of right whales to distinguish between two very similar classes of stimuli. What was intended to be a single group of SAG stimuli was in reality two separate sets of SAG recordings from two different species (North Atlantic and Southern right whales). Female North Atlantic right whales only approached Southern right whale playbacks, indicating that they were able to make this distinction. Males approached both types of stimuli, therefore it is not possible to say whether males were able to distinguish between the stimuli or just were less selective in what sounds they would approach. Even if males were able to detect a difference, with an operational sex ratio estimated at $4: 1$ and a low cost of mating relative to females, males should be expected to expend energy on any potential for mating. What acoustic difference the female right whales used to distinguish these calls from the other North Atlantic right whale stimuli remains in question. Only further playback studies will be able to distinguish between the proposed explanations of detectable species differences in calls including: 1) individual recognition allowing for the detection of an unknown individual in the population and 2) seasonal differences in SAG call production between the calving grounds (South Atlantic right whale recordings) and the feeding grounds (North Atlantic right whale recordings). A paired playback experiment to both North and South Atlantic right whale populations using three classes of stimuli (North Atlantic SAGs, South Atlantic SAGs, and synthetic calls) would allow one to distinguish between species differences and novelty in the response to these experiments. If female whales approach the synthetic calls and the calls of the other species, this would suggest that the females are responding to the call of a new individual. If female whales only approach the calls of the other species, this would provide more support for the idea of species differences. 
The playback experiments also raised more questions about the perceptual ability of right whales. Right whales responded to playbacks of Scream calls with rapid, direct-line approach, indicating their ability to use sound to accurately locate these groups. The persistence of these whales in searching for these sources indicates excellent spatial memory and a high motivation to find the sound source. Both males and females showed extensive searching behavior around the playback source after some playbacks had ended.

Additional data about the subsurface response of whales to the playbacks come from tag data. Behavioral results from DTAG work looking at right whale response to vessel approach, fortuitously yielded additional information about approach behavior to SAGs (Appendix 3). Individual whales showed subsurface orientation responses toward the playback, or multiple directional course corrections subsurface to orient toward a sound source.

Sound reception and localization are vital to mediate important behaviors in this species. Given the theoretical hearing range for right whales, there is an indication that anthropogenic sources of noise, particular ship traffic, may mask the most critical frequency range for communication. The frequency range for most SAG calls ranges from $50-16000 \mathrm{~Hz}$ with harmonics, but the majority of the energy for the calls is between $100-2500 \mathrm{~Hz}$. Further research needs to be carried out to estimate the effective range of communication for this species to determine how large a threat increasing noise levels may be to their ability to communicate and find mates.

The recordings of Scream sound production in SAGs and playback studies show that individual recognition from acoustic cues may be possible. Some individuals approached the same stimuli on multiple occasions, but did not approach other stimuli, even when the whale was sighted close to the playback vessel. Further, it is possible that the novelty of "new" whales in the population from the Southern right whale playback is what attracted females and juveniles to these playbacks. More repeated recordings from individuals might show that other call types, such as the Upcall, are also individually distinct. 
Several groups are proposing to use passive acoustic monitoring to determine right whale presence in areas of heavy shipping traffic or fishing effort. These passive monitoring efforts might also allow for tracking or detection of specific individuals that may be missed by traditional survey effort. This highlights the importance of further research to allow us to track individuals passively.

This thesis only collected data on sound production in SAGs in the Bay of Fundy on the summer feeding grounds. The discussion of the playback experiments suggests that seasonality may have been a distinguishing factor between the North and South Atlantic recordings. Recordings of SAGs in other habitats may reveal different calling behavior associated with particular group compositions. In particular, only one confirmed recording was made from an all-male group, and no recordings were made from an allfemale group. Recordings of these group types and of all SAGs in other areas will be required for full comparison of SAG behaviors in the different critical habitat regions.

The Gunshot sound described in Chapter 3 of this thesis remains a great mystery in the balaenid whales. Only briefly described in a handful of publications (Clark, 1983; Würsig \& Clark, 1993), this sound is arguably produced as frequently as the Scream calls from SAGs and in multiple behavioral contexts. The sound is intense and appears to be directional from recordings of lone males (Appendix 2). The mechanism for the production of the sound appears to be internal. Balaenid whales may also have specialized structures to protect their hearing from the potentially damaging effects of receiving these intense, sudden sharp onset, sounds which are $20 \mathrm{~dB}$ higher in SL than other SAG sounds (Chapter 3). Behavioral work to fully describe the use of this sound, may help shed light on the ultimate question of its function in this species.

The anatomical work on right whale inner ears and modeling of hearing also produced useful results that can inform future research efforts. The width and thinness of the baleen whale basilar membrane require careful preservation and decalcification of specimens. Efforts to decalcify the bones should rely not on trichloroacetic acid digestion, but rather a chelating agent such at EDTA. While this substantially increases the decalcification time, any other preparation method results in the loss of the thinner 
sections of the membrane. The right whale ear appears to be equipped with a massive stapedial muscle and a potentially functional ossicular chain. The relative size of the stapedial muscle in other baleen whales should be considered to investigate the role that this muscle might play in protecting the inner ear from loud sounds.

Studies of baleen whale acoustic communication still remain relatively primitive. The detailed questions being asked in several odontocete species (such as individual repertoires, perception, echolocating abilities and vocal learning to name a few) still remain challenging in baleen whale research. The increasing number of long term studies on baleen whale populations and development of increasingly sophisticated tools of recording sound production in the wild (Johnson \& Tyack, 2003), should allow for more detailed, controlled studies of baleen whale communication in the future. These studies may require creative ways of obtaining detailed focal follows with broad scale monitoring over tens or hundreds of miles to keep track of both the sender and receiver in baleen whale interactions. The number of questions raised by my thesis project suggests that many surprising and wonderful things remain to be discovered in the realm of baleen whale communication. I can only hope that the coming years bring rapid progress in this field to allow us not only to better understand these awe inspiring creatures, but to help us protect and promote their future on this planet. 


\subsection{SUMMARY OF THESIS CONTRIBUTIONS}

\section{Chapter 1: Introduction}

- A review of current knowledge about social behavior in baleen whales and the suggestion that long-term bonds may have been overlooked in many species.

\section{Chapter 2: Social Behavior in Right Whales}

- SAGs have been sighted in 11 out of 12 months and in all known North Atlantic right whale habitats.

- SAGs have variable group compositions including potentially reproductive and non-reproductive group compositions.

- There is an apparent seasonal trend for potentially reproductive SAGs increasing in the late summer and early fall.

- On average, males are seen twice as frequently as females in SAGs.

- Juvenile right whales are commonly sighted in SAGs.

- There is a strong correlation between number of pregnant females and number of SAGs observed, but no correlation between the number of calves observed and the number of SAGs observed when those calves would have been conceived.

\section{Chapter 3: Sound Production in SAGs}

- A variety of sound types are produced by right whales in SAGs

- Screams and gunshots are produced in bouts that correspond to the surfacing behavior of the SAGs.

- Different individuals produced particular sound types:

- Females form and maintain the groups by producing Scream calls.

- Males produce Upcalls to advertise their presence as they come into a group or when the female dives.

- Males may use Gunshot sounds as a threat signal to other males in the group or as a potential reproductive advertisement signal to the female.

- Some calves produce Warble sounds, similar to the Scream calls produced by females in SAGs. Warble sounds may be limited to female calves and provide evidence for the ontogeny of vocal development in a baleen whale.

- Source levels of Screams, Warbles, Noisy Blows and Gunshots in SAGs are comparable to source levels reported for other baleen whale species.

- Source levels for whale watching and research vessels near SAGs are approximately $10 \mathrm{~dB}$ re $1 \mu \mathrm{Pa}$ higher than the whale sounds..

- There is a correlation between source level of right whale sounds in the 2000$2200 \mathrm{~Hz}$ frequency band and noise level with higher source levels produced at times with higher noise. 


\section{Chapter 4: Playbacks}

- Right whales approach playbacks of SAG Scream calls but no whales approached Gunshot playbacks.

- Males approach playback of North Atlantic SAG Screams.

- Males and females approach playback of South Atlantic SAG Screams.

- Right whales show good acoustic localization abilities and spatial memory of sounds for up to at least 20 minutes.

\section{Chapter 5: Hearing}

- North Atlantic right whale ear anatomy is consistent with descriptions of other baleen whale ears.

- Right whale cochlear length scales with adult body size.

- Acid decalcification should not be used in histological preparation of baleen whale ears.

- Right whales show evidence of large numbers of ganglion cells compared to terrestrial mammals.

- North Atlantic right whale inner ear anatomy predicts a hearing frequency range $(10 \mathrm{~Hz}-22 \mathrm{kHz})$ that encompasses the frequency range of sounds produced by right whales.

\subsection{LITERATURE CITED}

Berta, A. \& Sumich, J. L. 1999. Marine mammals: Evolutionary biology. San Diego: Academic Press.

Best, P. B. 1994. Seasonality of reproduction and the length of gestation in southern right whales Eubalaena australis. Journal of Zoology, London, 232, 175-189.

Brownell, R. L. J. \& Ralls, K. 1986. Potential for sperm competition in baleen whales. Rep. Int. Whal. Commn. (Special Issue 8), 97-112.

Clark, C. W. 1983. Acoustic communication and behavior of the southern right whale. In: Behavior and communication in whales. American Association for the Advancement of Science Selected Symposium 76 (Ed. by Payne, R. S.), pp. 163198. Boulder, CO.: Westview Press.

Desharnais, F., Laurinolli, M., Hay, A. \& Theriault, J. A. 2000. A scenario for right whale detection in the Bay of Fundy. In: Oceans 2000, pp. 1735-1742.

Providence, RI.

Everitt, R. D. \& Krogman, B. D. 1979. Sexual behavior of bowhead whales observed off the north coast of Alaska. Arctic, 32, 277-280. 
Hasegawa, M., Adachi, J. \& Milinkovitch, M. C. 1997. Novel phylogeny of whales supported by total molecular evidence. J. Mol. Evol., 44, S117-S120.

Johnson, M. P.\& Tyack, P. L. 2003. A digital acoustic recording tag for measuring the response of wild marine mammals to sound. IEEE Journal of Oceanic Engineering, 28, 3-12.

Kraus, S. D. \& Hatch, J. J. 2001, Mating strategies in the North Atlantic right whale (Eubalaena glacialis). The Journal of Cetacean Research and Management (Special issue) 2, 237-244.

Norris, K. S., Villa-Ramirez, B., Nichols, G., Würsig, B. \& Miller, K. 1983. Lagoon entrance and other aggregations of gray whales (Eschrichtius robustus). In: Communication and behavior of whales (Ed. by Payne, R.), pp. 259-293. Boulder, CO: Westview Press.

Richardson, W. J., Finley, K. J., Miller, G. W., Davis, R. A. \& Koski, W. R. 1995. Feeding, social and migration behavior of bowhead whales, Balaena mysticetus, in Baffin Bay vs. the Beaufort Sea - regions with different amounts of human activity. Mar. Mam. Sci, 11, 1-45.

Rolland, R. M., Hunt, K. E., Wasser, S. K. \& Kraus, S. D. 2002. Reproductive and stress hormones in right whales. In: 2002 North Atlantic right whale consortium meeting (Ed. by M.Marx). New Bedford, MA.

Swartz, S. L. 1986. Gray whale migratory, social and breeding behavior. Report of the International Whaling Commission, Special Issue 8, 207-229.

Würsig, B. \& Clark, C. W. 1993. Behavior. In: The bowhead whale (Ed. by Burns, J. J., Montague, J. J. \& Cowles, C. J.), pp. 157-199. Lawrence, KS. 


\section{APPENDIX 1. SURFACE ACTIVE GROUP (SAG) SIGHTING HISTORY OF TWO FEMALES AND TWO MALES FROM 1982-2001 TO ILLUSTRATE INDIVIDUAL DIFFERENCES.}

Data from the North Atlantic right whale consortium. Photographs from the New England Aquarium

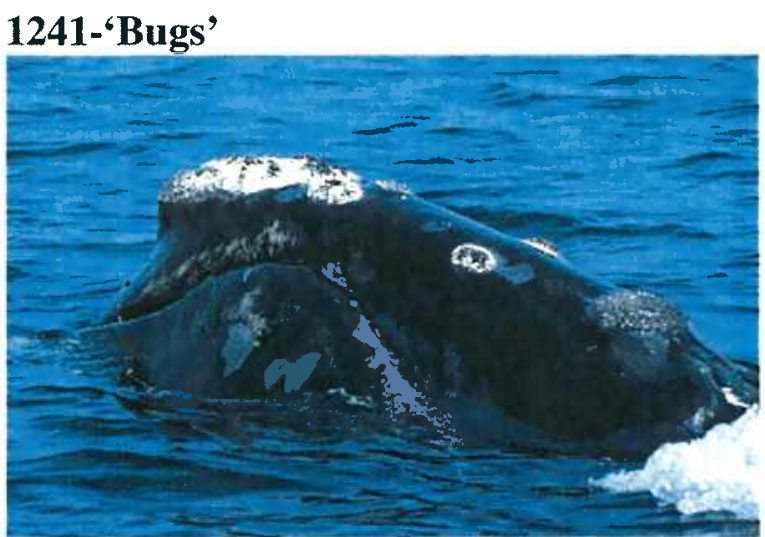

Female born in 1982. She has been sighted 149 times since her birth. Her first SAG sighting was in 1982 when she was 8 months of age. She has been seen in 12 SAGs. Once as a calf, three times as a two year old, and then there is a gap in SAG sightings until she was 9 years old in 1991 . She was seen as the focal female in a SAG in the BOF in 1994, while pregnant as the focal animal. She had her first calf at the age 7 in 1989. She has given birth to three calves at 6 and 7 year intervals in 1989, 1995, and 2002.

\section{7}

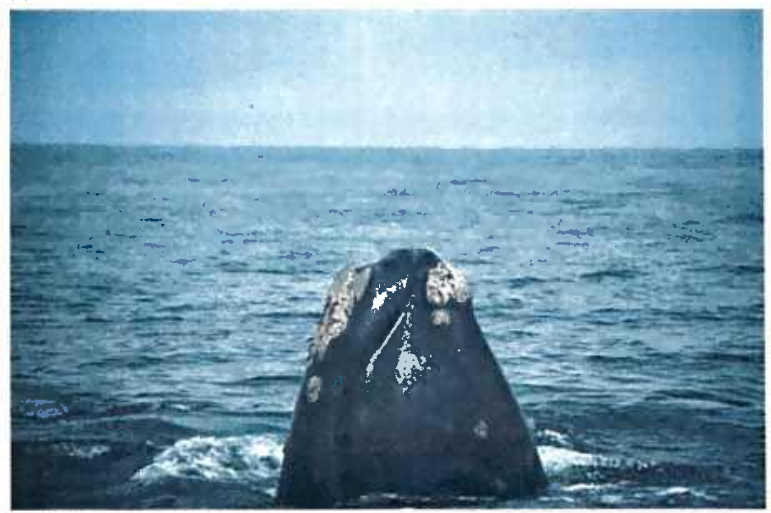

Female born in 1982. She has been sighted 105 times since her birth. Her first sighting in a SAG came in 1986 when she was $41 / 2$ years old. She has been seen in SAGs 36 times. This is one of the highest female percentage of sightings in SAGs. She has never been sighted with a calf. She has traveled down to the calving grounds on three occasions in 1990, 1996 and 2000, similar to the calving intervals seen in 1241 . She may have lost calves in these three years. She is the same matriline and haplotype as 1241 .

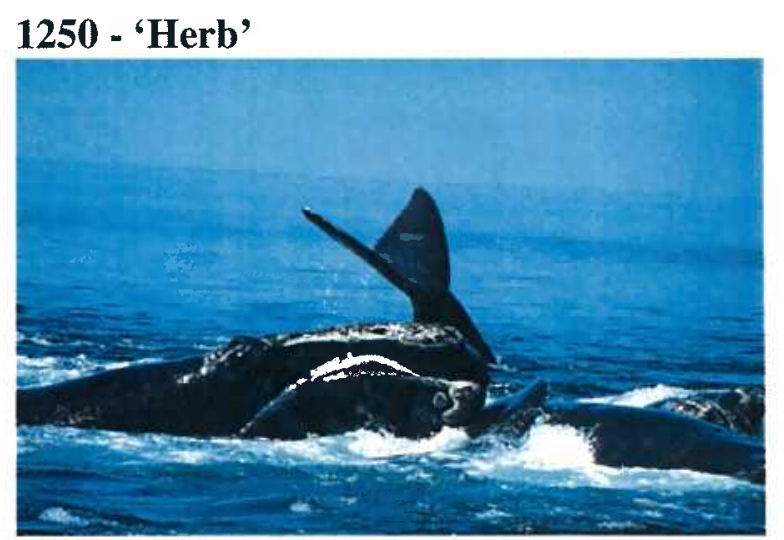

Male born in 1982. He has been sighted 86 times since his birth. His first SAG sighting was in 1984 when he was $21 / 2$ years old. He has been seen in 40 SAGs. He has traveled down to the calving grounds in the Southeastern U.S. 4 times since his calving year. His participation in SAGs has been in all regions, including the Southeastern U.S.

\section{9. 'Lacrosse'}

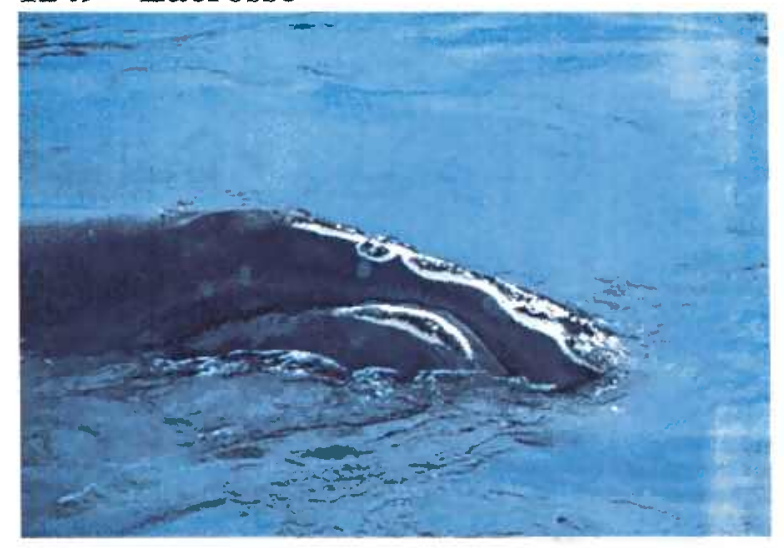

Male born in 1982. He has been sighted 95 times since his birth. His first SAG sighting was in 1985 when he was 3 years old. He has been seen in SAGs 30 times. He has only been sighted on the calving grounds once, in 2001 when he was 19 years old. All of his SAGs have been in BOF, $\mathrm{BB}, \mathrm{MB}$. 
Appendix 1: SAG sightings 


\section{APPENDIX 2. LONE WHALES PRODUCING GUNSHOT SOUNDS}

\section{A2.1 INTRODUCTION}

The Gunshot sound described in Chapter 3 is a distinct sound type that has received very little attention in the literature. Gunshot sounds, characterized by a very short intense broadband two part cracking sound sounding like a gunshot or a the crack of a whip, have been recorded in the presence of surface active groups (SAGs) and from lone animals in the population. Gunshot sounds have been documented in all Balaenid species, including Southern right whales Eubalaena australis (Clark, 1983), North Atlantic right whales Eubalaena glacialis, and the Bowhead whale Balaena mysticetus (Würsig \& Clark, 1993). Very little background information exists for the gunshot sound produced by right whales. These sounds were described by Clark (1983) as underwater slap sounds and documented both in single whales and in groups (Clark, 1983). The mechanism of sound production remains a mystery, but close visual observations of North Atlantic right whales during sound production do not indicate any body movement during production of the sound, suggesting an internal mechanism. It is important to investigate the characteristics of these sounds and their production in both SAGs and lone animals to better understand their function in these species. This appendix describes recordings of gunshot sounds from three lone adult male right whales in the Bay of Fundy between 2000 and 2001.

\section{A2.2 METHODS}

Recordings were made using a SONY DCR-TRV900 MiniDV video camera. A single Hi-Tech HTI-94-SSQ hydrophone (nominal frequency response $2 \mathrm{~Hz}-20 \mathrm{kHz}$ ) was connected to the video camera to make simultaneous synchronized audio and video recordings. Lone whales seen in the Bay displaying unusual and stereotyped surfacing behavior were seen on three occasions. The "typical" surface behaviors of lone adult whales in the Bay consist of slow straight line swimming and breathing, stationary 
breathing, or rapid swimming and rapid breathing (thought to represent respectively, recovery from feeding, resting, or SAG approach). The "unusual" surface behavior consisted of head lifts and long slow high arching dives in one location. When such a whale was sighted, recordings were made with a single hydrophone to determined if gunshot sounds were being produced. In each case described here, no other whales were visible in the area during the entire recording period and the sounds being recorded were of high intensity, suggesting that these individuals produced all sounds. The video was then viewed to determine timing of gunshot sound production, respiration, and surfacing and surface behaviors in each of these individuals.

\section{A2.3 Results}

Three adult male right whales were observed producing gunshot displays in the Bay of Fundy between 2000 and 2002. There was no systematic search for whales making this display. Two of these three whales were noted for their unusual surfacing behavior during photo $\mathrm{ID}$ surveys in 2000 and recordings were made. In 2002 , the recording was the result of hearing distant gunshot sounds and searching until we found a single whale in the area engaged in unusual surface behavior. The whale observed in 2002 was the same male that had been observed in a SAG with one adult female $1.5 \mathrm{~nm}$ away and 30 minutes prior to the observations made during Gunshot production. Table A2.1 summarizes the sighting information for each of these observations. Table A2.2 shows that no Gunshot sounds were recorded while the whale was breathing.

Table A2.1. Table showing the date, time, location, duration of observations and total number of gunshot sounds recorded.

\begin{tabular}{|c|c|c|c|c|c|c|c|}
\hline Date & Time & Location & $E G N O$ & Sex & Age & $\begin{array}{l}\text { Duration of } \\
\text { observations }\end{array}$ & $\begin{array}{c}\text { Number } \\
\text { of } G S \\
\text { recorded }\end{array}$ \\
\hline $8 / 11 / 2000$ & $15: 03$ & $\begin{array}{l}4430.5 / \\
6629.5\end{array}$ & 1424 & $M$ & $\begin{array}{l}\text { Adult } \\
(17+)\end{array}$ & 43 & 98 \\
\hline $9 / 6 / 2000$ & $15: 27$ & $\begin{array}{c}4433.4 / \\
6633.5\end{array}$ & 1307 & $\mathrm{M}$ & $\begin{array}{l}\text { Adult } \\
(18+)\end{array}$ & 17 & 44 \\
\hline $9 / 17 / 2002$ & $14: 43$ & $\begin{array}{c}4437.9 / \\
6625.2\end{array}$ & 1167 & $\mathrm{M}$ & $\begin{array}{l}\text { Adult } \\
(22+)\end{array}$ & 44 & 81 \\
\hline
\end{tabular}


Table A2.2. Number of gunshots, number of breaths visible on video, and the number of overlaps of breathing with gunshot sound production.

\begin{tabular}{c|ccc}
\hline Date & Number of Gunshots & $\begin{array}{c}\text { Number of breaths } \\
\text { visible on video }\end{array}$ & $\begin{array}{c}\text { Number of gunshots } \\
\text { while breathing }\end{array}$ \\
\hline $8 / 11 / 2000$ & 98 & 14 & 0 \\
$9 / 6 / 2000$ & 44 & 11 & 0 \\
$9 / 17 / 2002$ & 81 & 17 & 0 \\
\hline
\end{tabular}

Figures A2.1 and A2.2 illustrate a sequence of Gunshot sounds and a sequence of high frequency Scream-like calls produced on 8/11/00. All three individuals showed similar patterns of sound production, with high rates of Gunshot sound production. The average inter-gunshot interval was 4 seconds for all three individuals within a bout). The time between bouts was similar for all three adults as well, with only 3-5 minutes between surfacings.

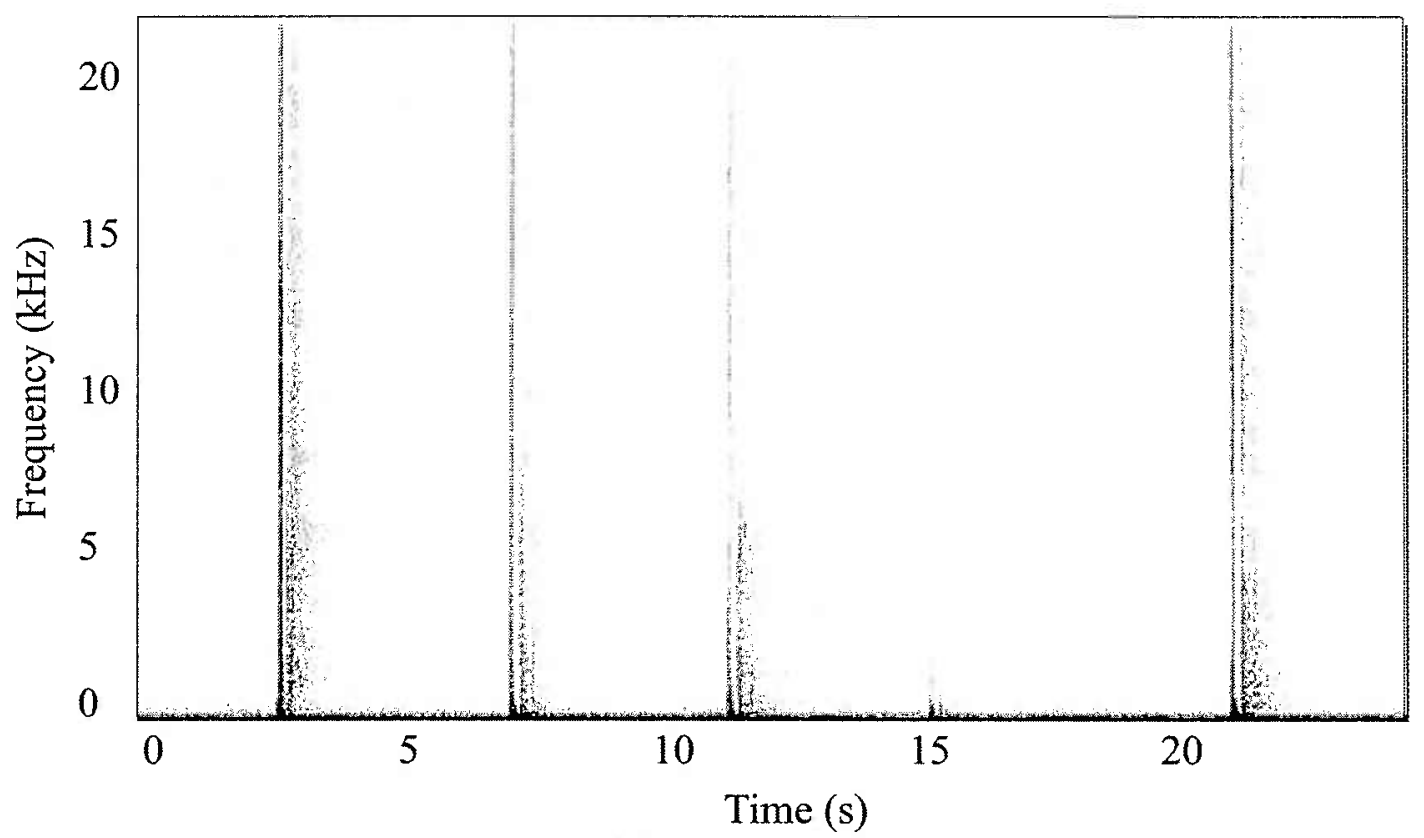

Figure A2.1. Spectrogram of a sequence of Gunshot sounds recorded on 8/11/00 from an adult male right whale (catalog number 1424). 


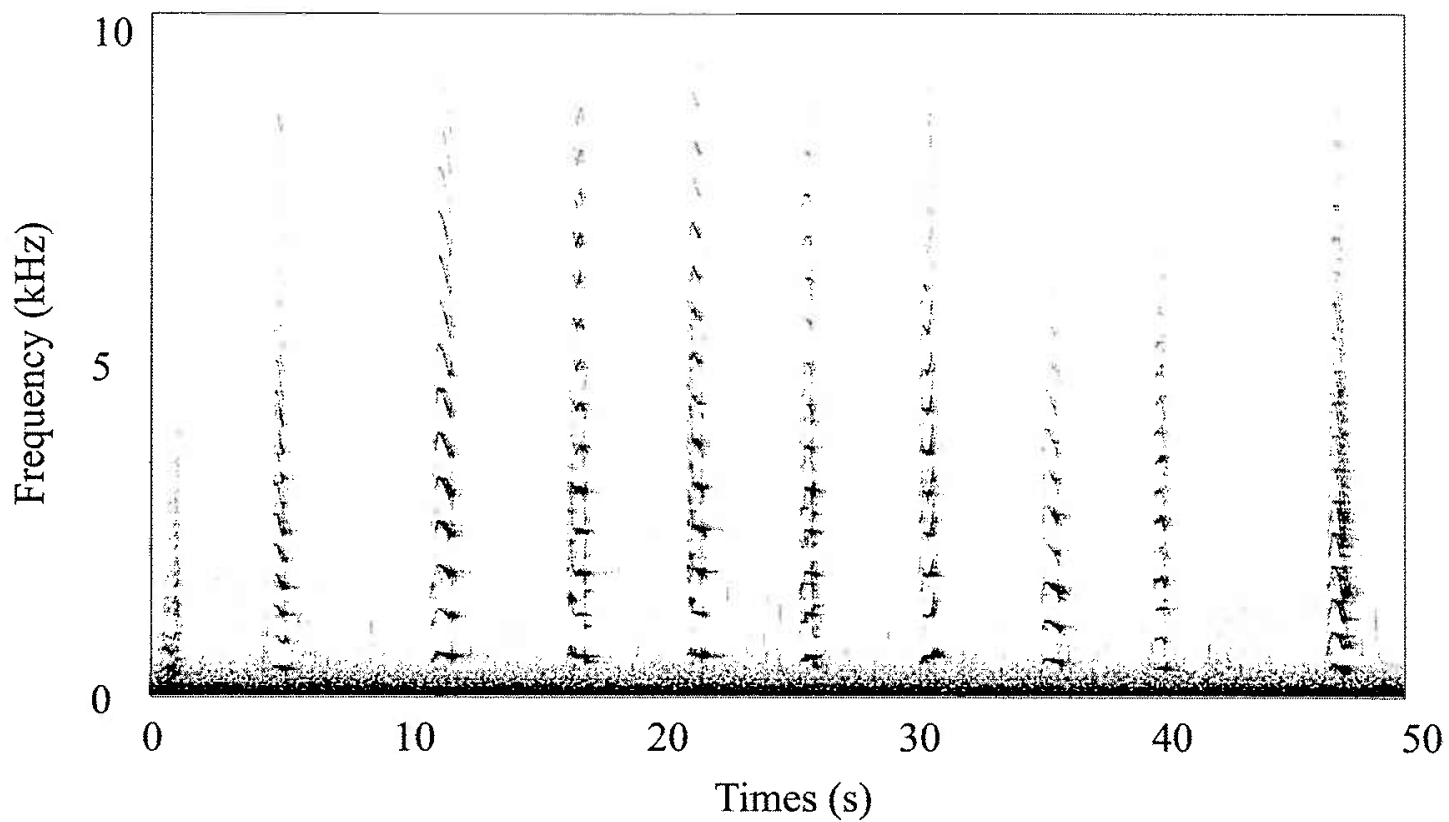

Figure A2.2. Example of Scream-like call production recorded on 8/11/00 from an adult male right whale (catalog number 1424). Note the first call in the sequence is an Upcall with multiple harmonics.

All three males exhibited similar behavior patterns during Gunshot sound production. Gunshots were primarily produced while the whale was at the surface, with a few produced immediately before surfacing or immediately after diving. Surfacing intervals ranged from 2-5 minutes, and dives were generally short (also 3-5 minutes). No whale was observed breathing when Gunshot sounds were recorded and no movement of flipper or tail appeared to coincide with the sound production. All three whales made head lifts (ranging from 1-3 head lifts per surfacing) where the head and chin were raised out of the whale with the rest of the whale's body approximately parallel to the water's surface. Most, but not all, head-lifts were followed by very loud, intense Gunshot sounds. Head-lifts were often made immediately before a dive. All three whales showed similar diving behavior. In each case, the male would raise the flukes high out of the water, with $1 / 4-2 / 3$ of the tail stock exposed to the air, and would sink slowly beneath the surface, with very little forward movement. Up calls, Down calls and Scream like calls were recorded in all three periods of observation. These tonal calls typically coincided with the dives of these three whales and were likely produced by the males. 
Each whale had some variation on this basic pattern of surfacing behavior (Figure A2.3). On 8/11/00, whale \# 1424 would arch his tailstock out of the water prior to the high fluke lift dive. On 9/6/00, whale \#1307, would flipper slap repeatedly (5-9 times) on each surfacing before rolling over and starting to produce gunshots. This observation made the acoustic differences between surface slaps and Gunshots very obvious. The 9/6/00 recording was made in sea state 3 , with the whale $200-300 \mathrm{~m}$ away. The flipper slaps were barely audible, but the Gunshot sounds were loud and intense from the same position. On 9/17/00, whale \# 1167, began to produce Gunshot sounds shortly after being involved in a SAG with a single mature female. On each surfacing, he would swim in slow circles at the surface while making occasional head lifts.

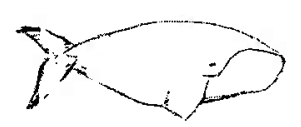

2)

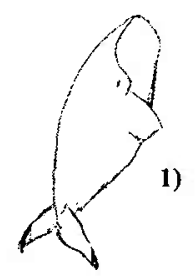

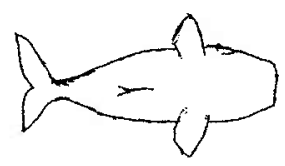

3)

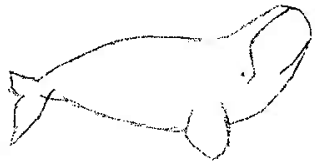

4)
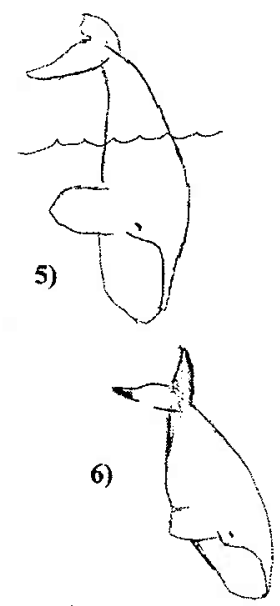

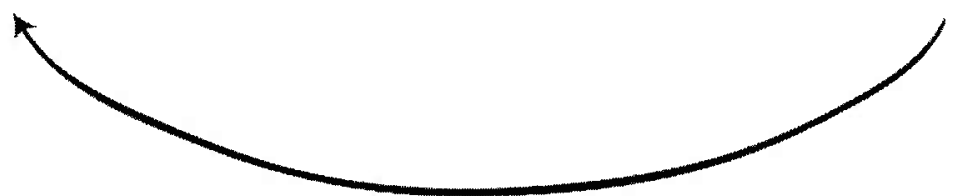

Figure A2.3. A typical surfacing sequence seen from adult male \#1307 on 9/6/00. The whale would 1) come to the surface, 3) then roll on his side and make flipper slaps, 4) make head lifts before his dive and 5) raise his flukes high out of the water before the dive. Then he would repeat the sequence on the next surfacing. The headlift high fluke raising behavior were observed in all three males.

\section{A2.4 Discussion}

Gunshot sounds are recorded both in SAGs and in lone males in the Bay of Fundy. Previous reports of female production of Gunshot sounds when her calf was threatened (Clark, 1983) have not been observed in North Atlantic right whales. Females may be capable of producing these sounds, but this has yet to be confirmed. It is clear 
that males can and do produce Gunshot sounds both in SAGs (Chapter 3) and alone. The Gunshot sounds produced in SAGs may very well fit the commonly assumed function as aggressive threat sounds (Würsig \& Clark, 1993). However, the aggressive function of Gunshot sounds produced by lone whales with no other right whales visible is less obvious. I suggest that Gunshot sounds serve a similar function to roaring in red deer, an acoustic signal that can be used in reproductive advertisement and harem defense (Clutton-Brock \& Albon, 1979; McComb, 1991). It is possible that these lone males are advertising their presence to females, similar to the female advertisement by Scream calls in SAGs.

It is interesting to note the similarities in these three observations. All the males are older adults, at least 17 years of age and possibly much older as all were first observed as adults in the early 80 's. The Gunshot displays were all produced in the early afternoon. Observations of SAG activity increasing in the evening may be related to this observation. Perhaps the mature males advertise until the evening SAG activity starts. It would be interesting to follow one of these males for the entire duration of the Gunshot display. The observation that whale \#1167 had been involved in a SAG immediately before his Gunshot display may lend credence to the suggestion that these displays are in some way tied to SAG behavior and reproductive advertisement.

Further research focusing on the Gunshot sound should be carried out to further describe this fascinating behavior that appears to be limited to the Balaenid whales. The mechanism for production and the functional use of this sound could aid in our understanding of the social structure in this species.

\section{A2.5 LITERATURE CITED}

Clark, C. W. 1983. Acoustic communication and behavior of the southern right whale. In: Behavior and communication in whales. American Association for the Advancement of Science Selected Symposium 76 (Ed. by Payne, R. S.), pp. 163198. Boulder, CO.: Westview Press.

Clutton-Brock, T. H. \& Albon, S. D. 1979. The roaring of red deer and the evolution of honest advertisement. Behaviour, 69, 145-169. 
McComb, K. 1991. Female choice for high roaring rates in red deer, Cervus elaphus. Animal Behaviour, 41, 79-88.

Würsig, B. \& Clark, C. W. 1993. Behavior. In: The Bowhead Whale (Ed. by Burns, J. J., Montague, J. J. \& Cowles, C. J.), pp. 157-199. Lawrence, KS. 
Appendix 2: Gunshots 


\section{APPENDIX 3. EXTENDED ABSTRACT FOR PRESENTATION AWARDED BEST STUDENT ORAL PRESENTATION AT THE 15TH ANNUAL CONFERENCE OF THE EUROPEAN CETACEAN SOCIETY: ROME, ITALY, MAY $6-10^{\mathrm{TH}}, 2001$.}

USE OF A DigItal ACOUSTIC TAG TO DOCUMENT RESPONSE OF THE NORTH ATLANTIC

RIGHT WHALE TO SURFACE ACTIVE GROUPS

Parks, S., Tyack, P.L., Johnson, M., Nowacek, D., Biassoni, N.

\section{A3.1 AbStract}

North Atlantic right whales (Eubalaena glacialis) perform their most conspicuous surface behaviors when involved in surface active groups (SAGs). These surface groups involve $2-20+$ whales engaged in social/sexual behavior at the surface and often produce particular vocalizations. Preliminary playbacks of vocalizations recorded from these groups have been shown to strongly attract adult male right whales. The digital acoustic recording tag (DTAG), logging both acoustic data from the environment and behavioral orientation data, has proven to be an excellent tool to further investigate acoustic communication used by right whales in the formation of these groups. The DTAG records ambient acoustic data as well as the whale's depth, pitch, roll, and heading. During the summer of 1999 and 2000, these suction cup tags were attached to right whales in the Bay of Fundy, Canada. Right whales responded to exposures of prerecorded right whale sounds from SAGs and to sounds produced by actual SAGs in the field. Two playbacks of recorded sound were carried out to tagged whales in 2000. Additionally, three tagged whales joined actual SAGs while carrying the tag. In both scenarios, the tag sensors recorded subtle, sub-surface responses to sound exposure. These responses included changes in heading and cessation of active swimming that reduced flow noise and made the playback more clearly audible on the tag record. Additional playbacks may be able to shed light on the effective range of communication in these whales by determining what received level of SAG vocalizations is necessary to elicit a response.

\section{A3.2 INTRODUCTION}

Right whales

North Atlantic right whales (Eubalaena glacialis) are the most endangered baleen whales in the world. For centuries they were considered the "right" whales to hunt because of their abundant blubber and slow speeds. Even though these whales have been 
protected from whaling for much of this century, today fewer than 350 right whales remain in the North Atlantic (Knowlton et al., 1994; IWC, 2001). (Knowlton et al., 1994; IWC, 2001). North Atlantic right whales, unlike other populations of right whales have not shown significant signs of recovery after the end of whaling in the mid 1930s. The population growth rates for the southern hemisphere have been estimated at almost $7 \% /$ year (Best et al., 2001) while the North Atlantic right whale growth rates have declined from $3-5 \%$ in the 80 's to $1-2 \%$ in the 90 's (Kraus et al., 2001). Reducing human contributions to mortality is imperative for survival of this species (Caswell et al., 1999). There are two major anthropogenic threats to right whale survival, collisions with vessels and entanglement in fishing gear (Knowlton \& Kraus, 2001). A less obvious and potentially long-term threat comes from impacts of ever increasing levels of anthropogenic noise in the environment. It is important to understand how right whales use sound to communicate to be able to determine the potential impacts of increasing noise.

\section{Surface Active Groups}

One of the most common social interactions in right whales is the Surface Active Group (SAG). These groups are defined as two or more animals interacting at the surface, less than one body length apart with frequent physical contact (Kraus \& Hatch, 2001). The typical composition of these groups involves a single female at the center of the group flanked by males, referred to as the focal female (Figure A3.1). The males immediately next to the female, close enough to the female to achieve intromission, are termed alpha males. Males outside of the alpha males are termed beta males, and whales at the edge of the group are called peripheral males (Kraus \& Hatch, 2001). The males compete to gain access to the female in the alpha male position where intromission is possible (Kraus \& Hatch, 2001; Brownell, 1986). 

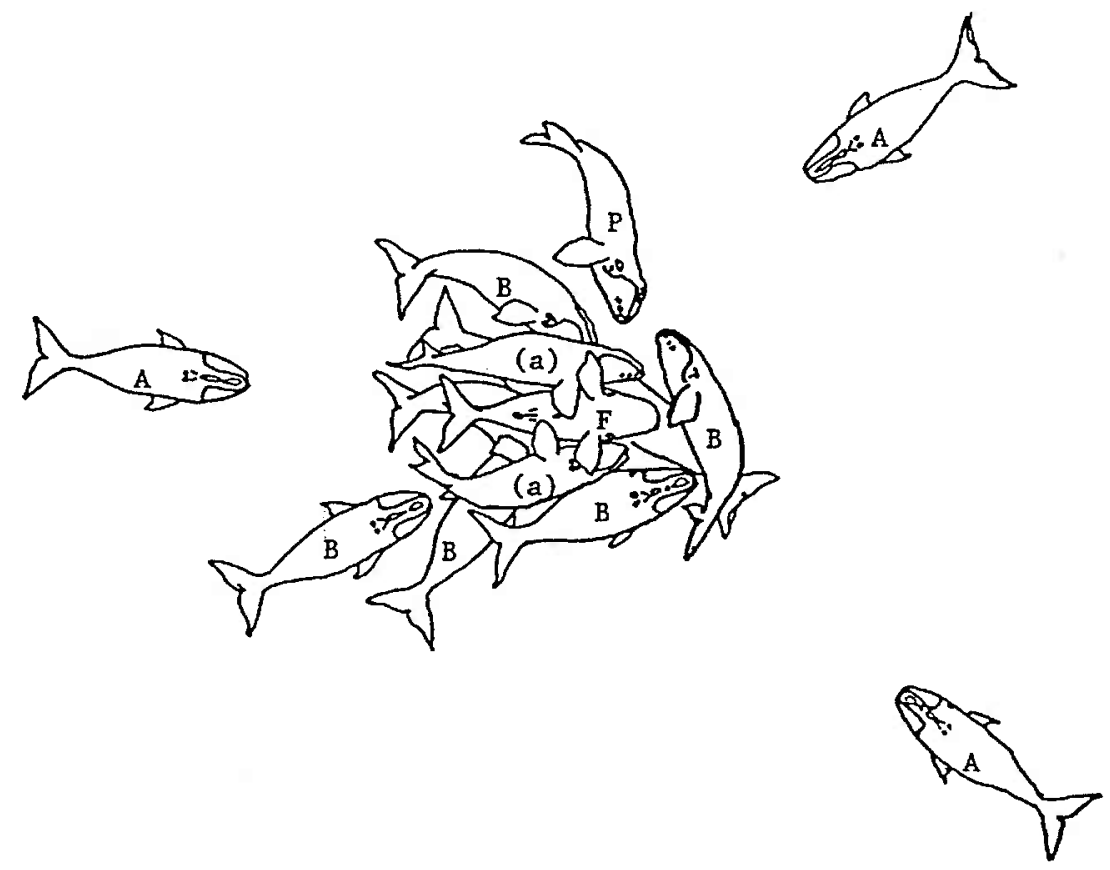

Figure A3.1. Figure from Kraus \& Hatch 2001 showing the typical formation of a surface active group. The focal animal (F) is at the center of the group, alpha whales next to the focal animal (a), beta whales (B), peripheral whales (P) and approachers (A) further out in the group.

Use of sound for communication

Because of limited visibility in the waters of the North Atlantic, sound is the most likely method employed by right whales to find conspecifics for mating. Particular vocalizations and sounds have been associated with SAGS. The calls recorded from 8 SAG's by the New England Aquarium last from $.5-2.8$ seconds, range in frequency from 400 to $3200 \mathrm{~Hz}$, and occur on average 12 per minute (Kraus \& Hatch, 2001). Figure A3.2 illustrates an example of five calls recorded from a SAG in the Bay of Fundy in 2000. These calls are believed to function to attract other whales to these groups. 


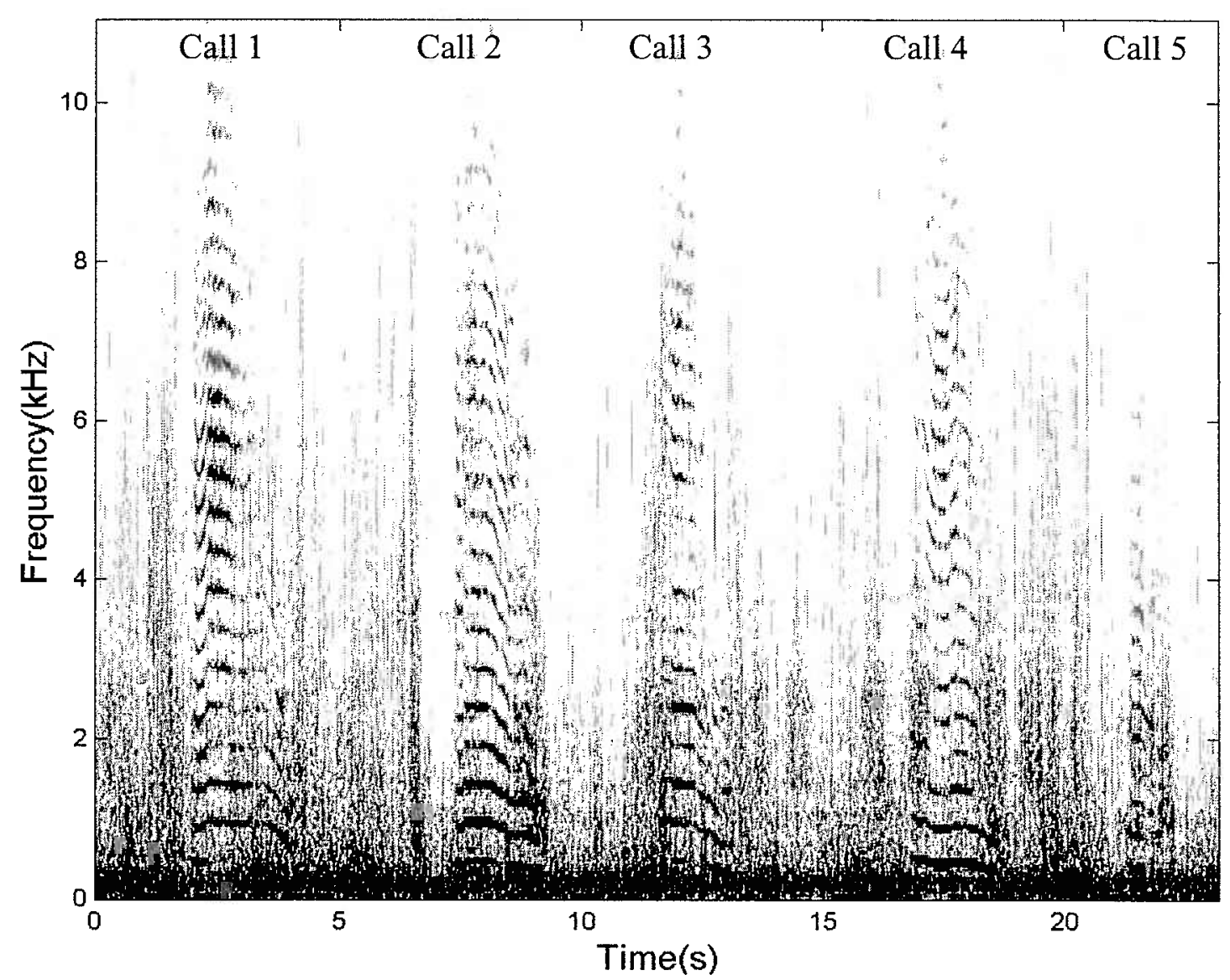

Figure A3.2. Spectrogram of a sequence of five Scream calls recorded from a Surface Active group in the Bay of Fundy.

The goal of this research was to document the responses of individual right whales to the sounds produced in SAGs by means of attaching a tag to monitor the acoustic environment and physical orientation of the whale. These responses were investigated both through playback experiments and by documenting the response of individuals to real SAGs in the field. It is hoped that these data will provide insight into the perceptual abilities of right whales on how they use sound to locate other right whales. 


\section{A3.3 MethodS}

This research was carried out in the Bay of Fundy, Canada, in the summers of 1999 and 2000. The research involved focal animal follows (Altmann, 1974) of individual right whales. These right whales were tagged with a suction-cup digital acoustic recording tag, DTAG (Johnson \& Tyack, 2003) for a study of responses of right whales to vessel approach. Playback of recordings from SAGs was used to demonstrate that right whales would respond to sounds. One tagged whale also approached and joined a SAG in progress and his approach response was recorded by the DTAG.

Behavioral observations

Focal follows were carried out on tagged right whales. At each surfacing, the time, bearing, range, heading, and behavior of the whale were documented. It often took several seconds to locate the whale after it surfaced, and the whale occasionally surfaced too far from the observation platform for observers to document behavior. The main goal of the behavioral observations was to provide a good surface position and heading of the whale to calibrate the tag readings. Therefore surface behavioral data were not consistently collected.

Playback trials

Playbacks were made using the protocol described in Parks (2003). A single stimulus was used for the playbacks to all tagged whales. The New England Aquarium recorded this stimulus on Brown's Bank in 1992. The playback stimulus was 20 minutes in duration.

\section{The DTAG}

The DTAG is a small, non-invasively attached unit that simultaneously records the acoustic environment with a hydrophone and the orientation of the whale using depth, temperature and orientation sensors (Johnson \& Tyack, 2003). The tags were non- 
invasively attached using suction-cup attachment. The tags were deployed from a small vessel using a cantilevered 12-m carbon fiber pole system designed by Michael Moore (Moore et al., 2001). The tag was attached dorsally, approximately midway between the blowholes and the caudal peduncle. Batteries, syntactic foam floatation, and a radio transmitter for tracking and package recovery were included in a thermoformed polyethylene hull to reduce drag (Figure A3.3).

The tag sensors provided a variety of data to be used in determining the response of whales to SAG sounds. The hydrophone recording allowed for detection of the playback or real SAG sounds at the whale. The accelerometers allowed for calculation of the fluke stroke rate of the whale to determine swimming behavioral responses (Nowacek et al., 2001). The sensors on the tag could be used to estimate the orientation of the whale, allowing for the construction of approximate underwater tracks of the animal using dead-reckoning (Johnson \& Tyack, 2003).

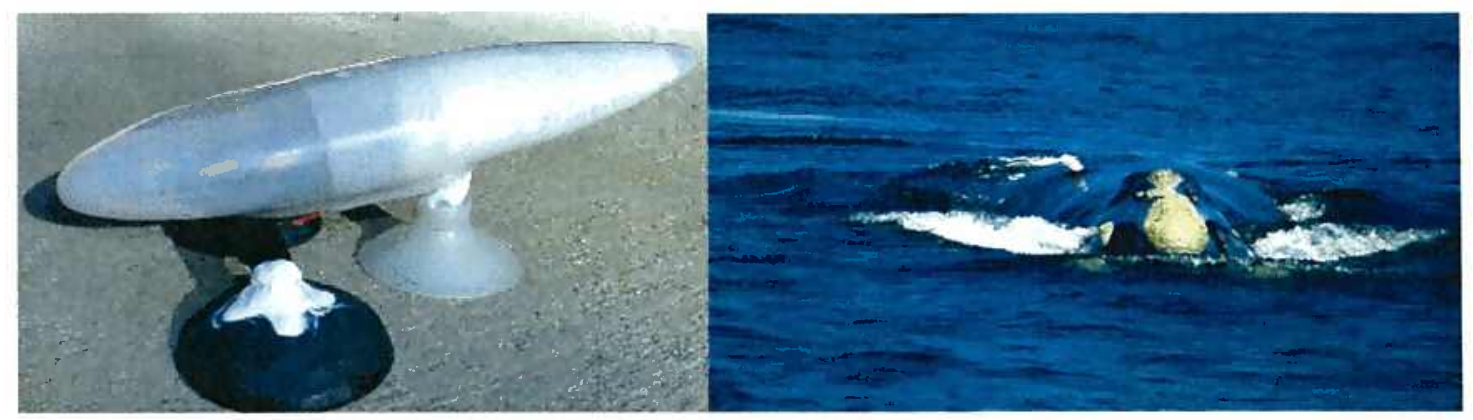

Figure A3.3. A) A photograph of the DTAG before attachment and B) the DTAG on a right whale. Photograph of the tag \& whale by K.A. Shorter.

\section{A3.4. Results}

Preliminary playback trial results indicated that whales responded to playbacks of SAG stimuli by approaching the playback source (Figure A3.4). 


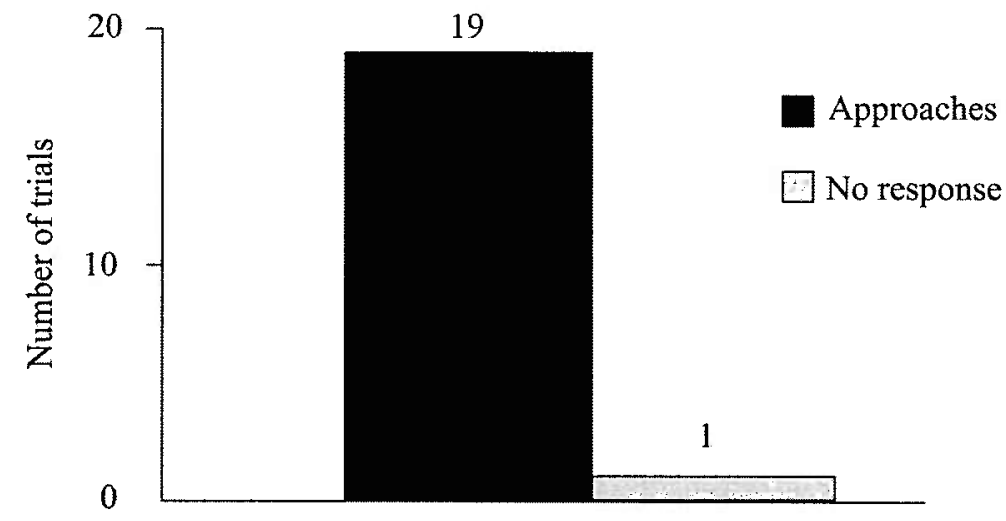

Figure A3.4. Approach vs. no response during SAG playback trials in 1999 and 2000. The single no response was from a tagged whale when a tanker was less than 1 mile away.

Playbacks to tagged whales

A single playback of SAG sounds was carried out to a tagged whale in 1999. A tanker was less than $1 \mathrm{~nm}$ from the whale during the time of the playback and the playback stimulus could not be heard on the hydrophone record from the tag. There was no observed response judging either from behavioral observations or the tag record. Two playbacks of SAG sounds were carried out to tagged whales in 2000. Additionally, one tagged whale joined an actual SAG while carrying the tag in 2000. For this paper, the response of these three whales tagged in 2000 will be considered (Table A3.1).

Table A3.1. The date and duration of attachment of tag to right whales in the Bay of Fundy in 2000. The playback to the whale was either of SAG sounds (W) or vessel noise (V).

\begin{tabular}{|c|c|c|c|c|}
\hline Date Tagged & $\begin{array}{l}\text { Whale } \\
\text { ID }\end{array}$ & $\begin{array}{c}\text { Duration of tag } \\
\text { attachment } \\
\text { (hr:min) }\end{array}$ & Playbacks & Response to Playback/Other \\
\hline $8 / 11 / 99$ & 1238 (Adult Male) & $8: 51$ & $W$ & No response \\
\hline $8 / 1 / 00$ & 1307 (Adult Male) & $20: 12$ & W & Cessation of swimming \\
\hline $8 / 5 / 00$ & $\begin{array}{l}2720 \text { (Unknown } \\
\text { age/sex) }\end{array}$ & $3: 40$ & $\mathrm{~W} / \mathrm{V}$ & $\begin{array}{l}\text { Roll \& temporary heading } \\
\text { change in response to W }\end{array}$ \\
\hline $8 / 9 / 00$ & $\begin{array}{l}2760 \text { (Juvenile } \\
\text { Male) }\end{array}$ & $1: 38$ & None & $\begin{array}{c}\text { Tag knocked off in SAG during } \\
\text { approach }\end{array}$ \\
\hline
\end{tabular}


Response of tagged whales

Figure A3.5 illustrates the response of the whale tagged on 8/1/00. The four panels (from the top) represent the simultaneous 1) depth of the whale in meters, 2) pitch angle of the tag in degrees 3) angular fluke rate in degrees/second and 4) spectrogram of the acoustic recording. This figure highlights two minutes of the tag record when the playback of SAG sounds started. Almost immediately after the start of the playback, there is a noticeable decline in the angular fluke rate and a reduction of the flow noise in the acoustic record. This indicates that the whale stopped swimming for approximately 20 seconds after the start of the playback. No other response was seen from this individual.

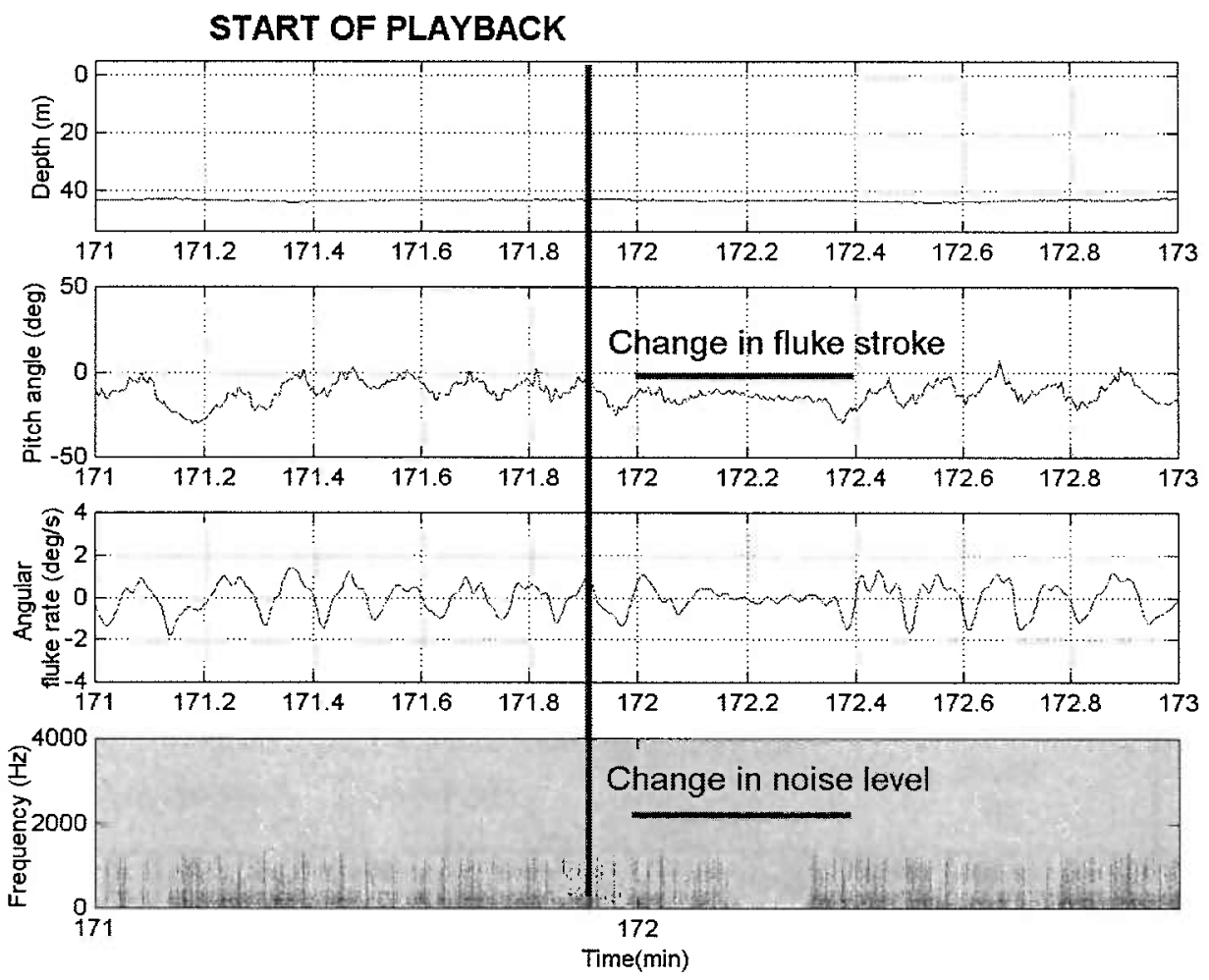

Figure A3.5. A plot showing response of a tagged right whale to a SAG playback. The depth, pitch, angular fluke stroke rate and acoustic record are included in the figure. 


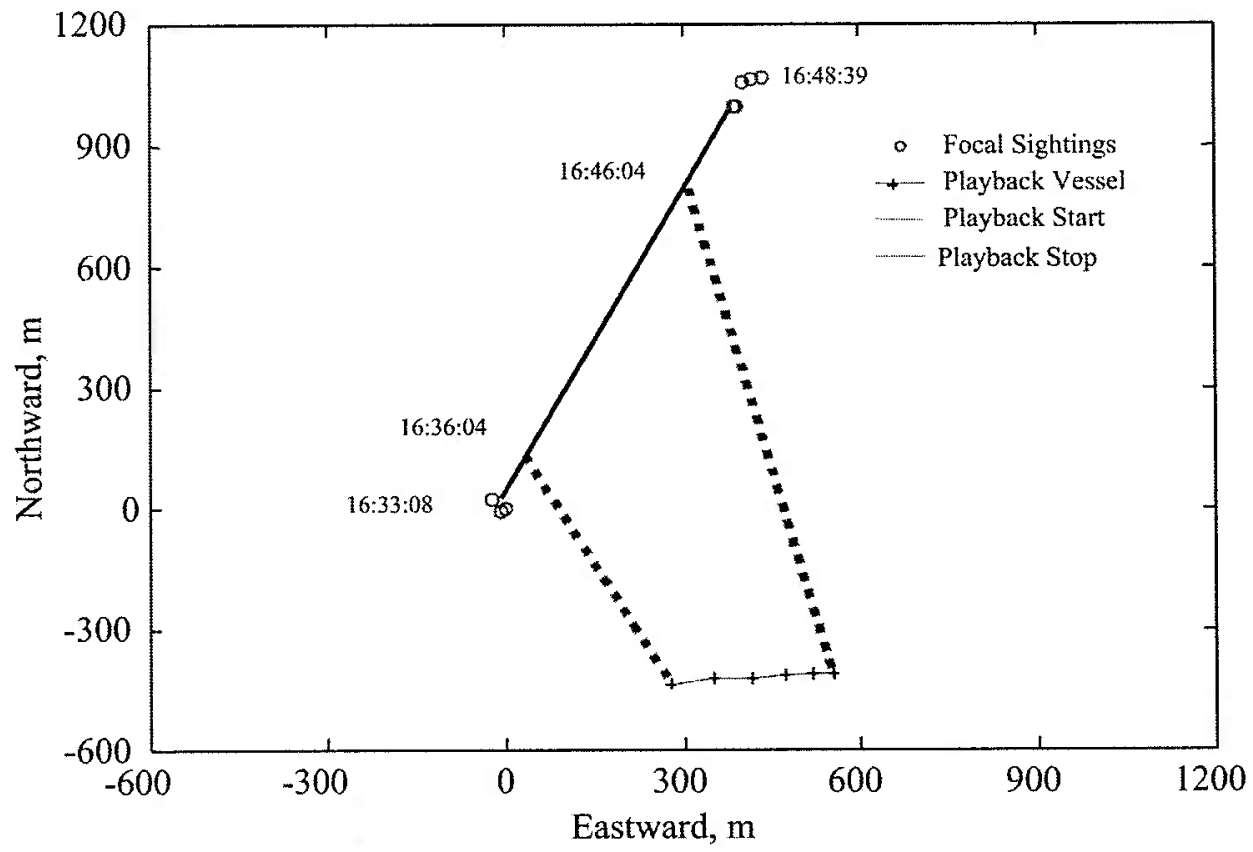

Figure A3.6. A plot showing surface observations of a right whale during a SAG playback experiment.

Figure A3.6 illustrates the surfacings of the whale tagged on 8/5/00 recorded from behavioral observations. The figure shows an $1800 \times 1800 \mathrm{~m}$ map. The open circles correspond to the position of sightings of the tagged whale from the observation vessel. The plus signs indicate the position of the playback vessel. The two dashed lines indicate the time of the start (green) and stop (red) of the playback. From the surface observations it looked as though there was no response from the whale as it swam away from the playback vessel, in the direction it had previously been heading. The whale joined a SAG approximately 20 minutes after the end of the playback.

Figure A3.7 shows the tag sensor data corresponding to the dive of the $8 / 5 / 00$ tagged whale illustrated in Figure A3.6. The top panel shows the depth of the whale in meters. The bottom panel shows the heading of the whale recorded by the tag. The open circles in both panels indicate the observed depth and heading of the whale from behavioral observations. Note the excellent correspondence between the two observations. The tag heading sensors indicate a change in orientation immediately after the start of the playback. The whale then returned to his original heading after 2 minutes. 


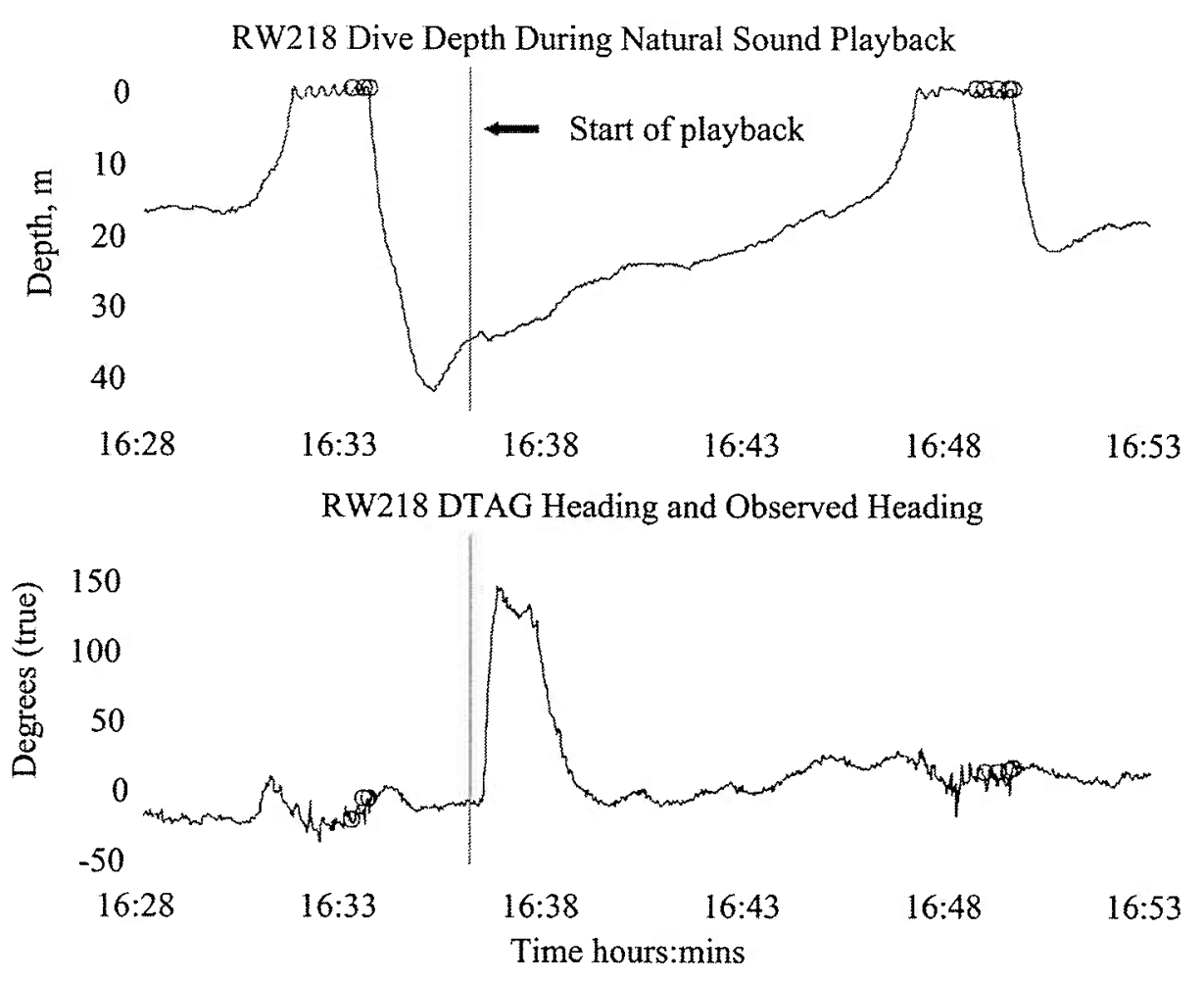

Figure A3.7. Figure showing observed depth and heading of the same whale from figure AIII.7 using the DTAG data.

Figure A3.8 is a dead-reckoned track of the whale subsurface, illustrating the change in heading toward the playback vessel before the whale turned away and continued in his original course. The figure represents the same 1800 x 1800 m map from Figure A3.6. The surface sightings, playback vessel and start and stop of the playback are still included in the figure. Now, the dead-reckoned track of the whale based on heading and depth data from the DTAG (using an constant estimated speed) are plotted on the graph. This plot shows that the whale turned toward the playback vessel after the start of the playback while he was on the bottom of a dive, before he turned back to the North. 


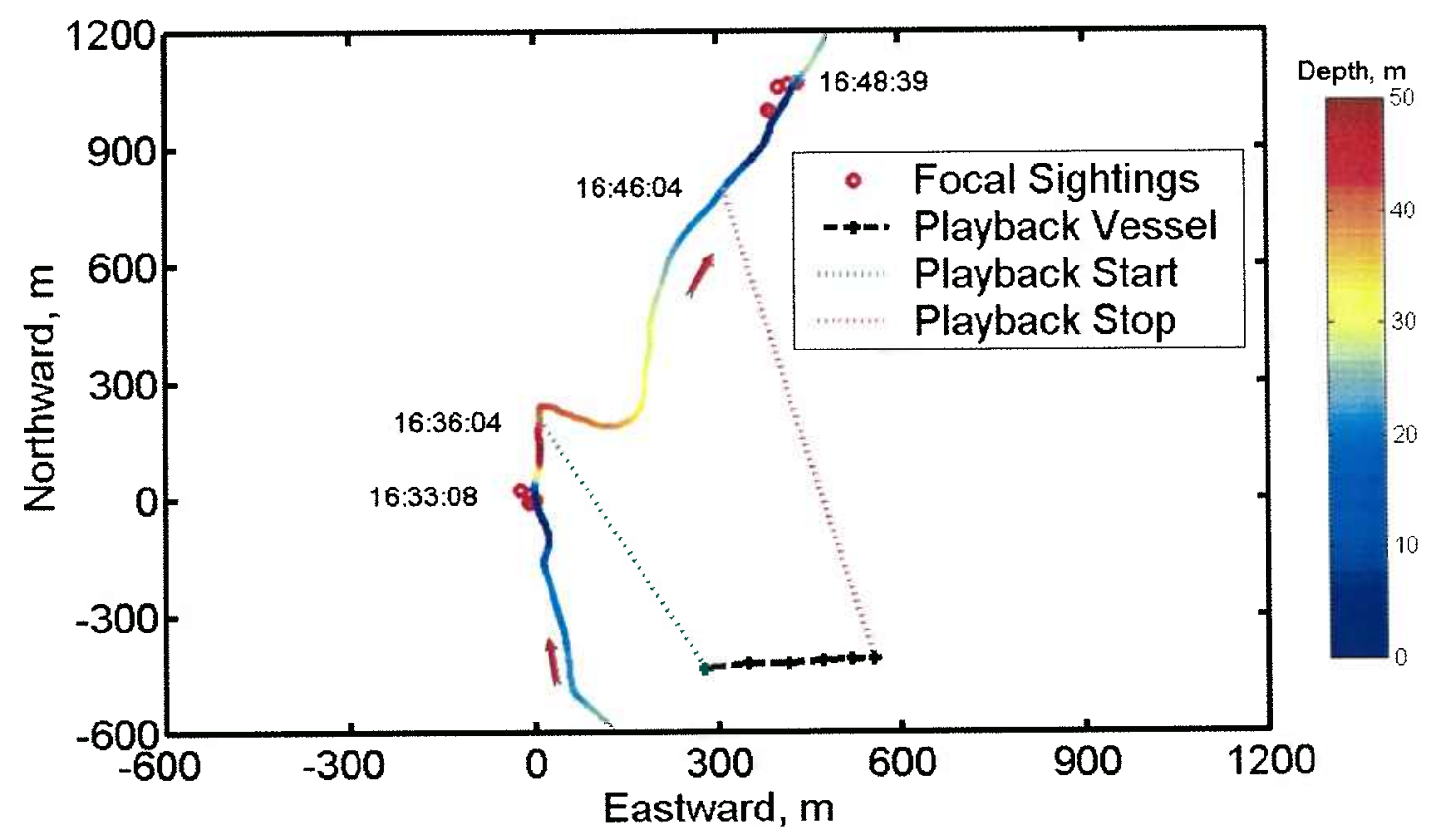

Figure A3.8. A combined pseudo-plot of the whale underwater during the playback trial, indicating that the whale turned toward the playback vessel while on a dive.

Figure A3.9 shows the dead-reckoned track of a juvenile male whale approaching a SAG on 8/9/00. The figure shows a 1200 x $1200 \mathrm{~m}$ map. The dead-reckoned subsurface track of the whale was calculated the same way the track was calculated for Figure A3.8 (Johnson \& Tyack, 2003). The surface observations of the whale's position are shown by the open circles. The stars indicate the time that SAG calls can be heard on the acoustic record from the tag. The whale appeared to orient toward the SAG after hearing each vocalization. The whale made several changes in heading immediately after a SAG call was recorded on the acoustic record from the tag. It took the whale 16 minutes to join the SAG after hearing the first call. 


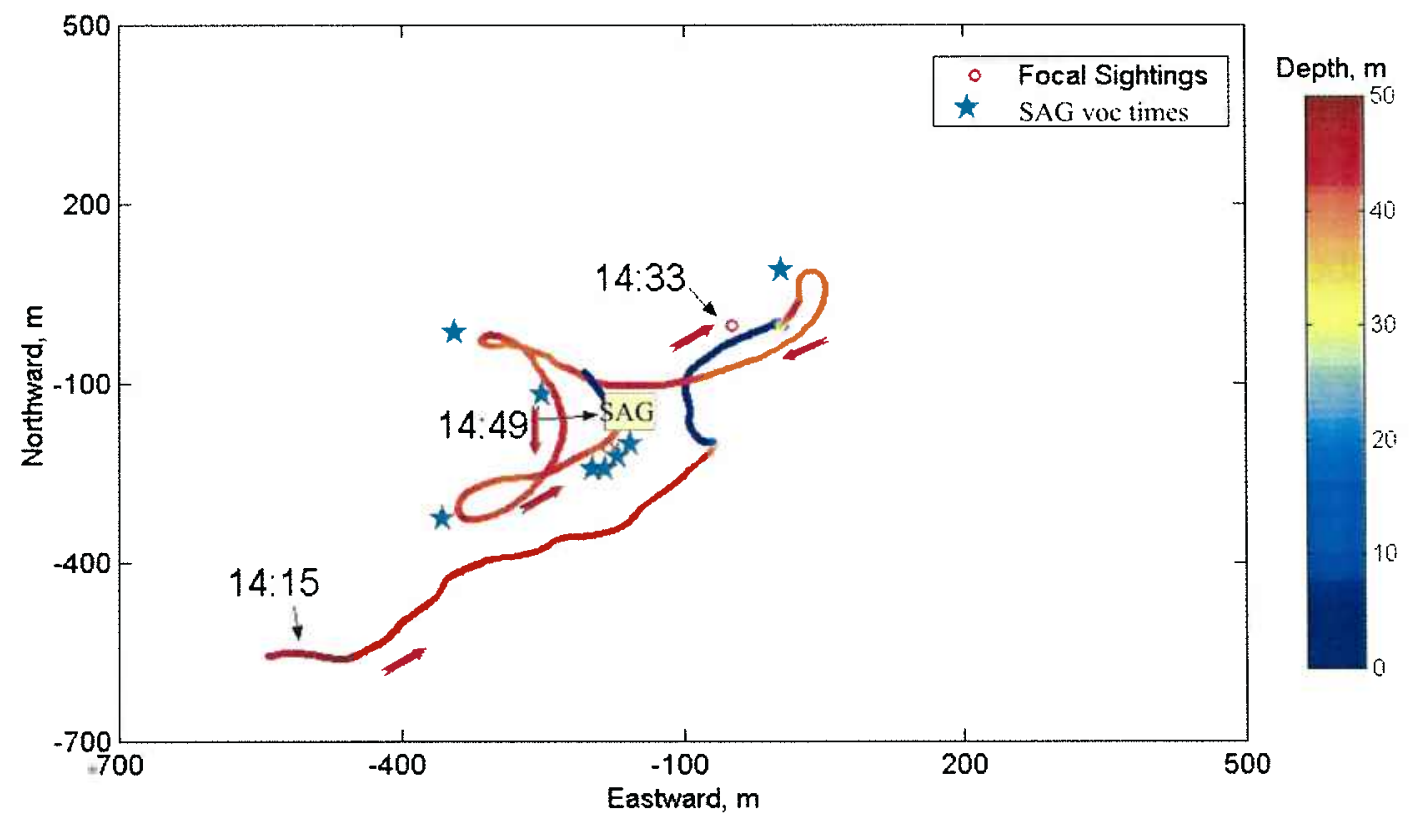

Figure A3.9. Dead-reckoned of the track of a young male approaching an actual surface active group. The stars indicate the time at which Scream calls were recorded on the DTAG.

\section{A3.5 Discussion}

The results presented here are just a small sample of the type of data that can be obtained from tagging of baleen whales. These three example cases indicate that right whales show a variety of responses to sounds heard in their environment. The cessation, or slowing of swimming seen in the whale from $8 / 1 / 00$ indicates that the flow noise generated from swimming may impair hearing in right whales, making it necessary for them to slow down or stop to hear faint sounds. This response may also represent a startle response on the part of the animal, as the whale stopped only briefly and did not approach the playback.

The orientation response seen in the whale from $8 / 5 / 00$ indicates that right whales are accurately able to determine the direction of received sounds. This example was particularly interesting because it illustrates the benefits of tag data. Surface observations led the observers to believe that the whale had not responded to the playback. The tag data indicated that the whale did hear and respond to the sound. This particular individual 
joined a SAG approximately 20 minutes after the end of the playback. The whale had been heading toward the SAG when the playback took place and may have chosen the original SAG over the playback stimuli.

The third example here, from the 8/9/00 tagging, illustrates a searching pattern by a juvenile male whale. The whale changed heading, correcting his course after every SAG call that he heard. This was a young whale, only 3 years of age at the time of the tagging. It is difficult to determine if his orientation and localization response (taking 16 minutes to find a SAG less than $200 \mathrm{~m}$ away) is representative of right whales. He may have been inexperienced or hesitant in approaching the SAG. He did not demonstrate surface racing diving behavior that often characterizes males approaching SAGs (Kraus \& Hatch, 2001).

These tags, coupled with more extensive observations and playbacks, could be used to investigate the localization and orientation response of right whale on a fine scale. Future research should be done to address these issues.

\section{A3.6 Literature Cited}

Altmann, J. 1974. Observational Study of Behavior: Sampling methods. Behaviour, 49, 227-267.

Best, P. B., Brandao, A. \& Butterworth, D. S. 2001. Demographic parameters of southern right whales off South Africa. Journal of Cetacean Research Management (Special Issue 2), 161-169.

Brownell, R. L. 1986. Potential for Sperm Competition in Baleen Whales. Rep. Int. Whal. Commn. (Special Issue 8), 97-112.

Caswell, H., Fujiwara, M. \& Brault, S. 1999. Declining survival probability threatens North Atlantic right whale. Proceedings of the National Academy of Science, 96, 3308-3313.

IWC. 2001. Report of the workshop on status and trends of western North Atlantic right whales. The Journal of Cetacean Research and Management (Special issue) 2, 61-87. 
Johnson, M. P. \& Tyack, P. L. 2003. A digital acoustic recording tag for measuring the response of wild marine mammals to sound. IEEE Journal of Oceanic Engineering, 28, 3-12.

Knowlton, A. R. \& Kraus, S. D. 2001. Mortality and serious injury of northern right whales (Eubalaena glacialis) in the western North Atlantic. Journal of Cetacean Research Management (Special Issue) 2, 193-208.

Knowlton, A. R., Kraus, S. D. \& Kenny, R. D. 1994. Reproduction in North Atlantic right whales (Eubalaena glacialis). Canadian Journal of Zoology, 72, 297-305.

Kraus, S. D., Hamilton, P. K., Kenney, R. D., Knowlton, A. R. \& Slay, C. K. 2001. Reproduction parameters of the North Atlantic right whale. The Journal of Cetacean Research and Management. (Special issue) 2, 231-236.

Kraus, S. D. \& Hatch, J. J. 2001. Mating strategies in the North Atlantic right whale (Eubalaena glacialis). The Journal of Cetacean Research and Management (Special issue) 2, 237-244.

Moore, M. J., Miller, C. A., Morss, M. S., Arthur, R., Lange, W., Prada, K. G., Marx, M. K. \& Frey, E. A. 2001. Ultrasonic measurement of blubber thickness in right whales. J. Cetacean Res. Manage., 2, 301-309.

Nowacek, D., Johnson, M. J., Tyack, P. L., Shorter, K. A., McLellan, W. A. \& Pabst, D. A. 2001. Bouyant Balaenids: the ups and downs of buoyancy in right whales. Proc. R. Soc. London B., 268, 1811-1816.

Parks, S. E. 2003. Response of North Atlantic right whales (Eubalaena glacialis) to playback of calls recorded from surface active groups in both the North and South Atlantic. Mar. Mam. Sci, 19.

\section{A3.7 ACKNOWLEDGEMENTS}

The DTAG project and right whale tagging was the work of Dr. Mark A. Johnson, Dr. Douglas Nowacek and Dr. Peter L. Tyack. A large number of people were involved in the tagging project and playback projects directly. The New England Aquarium research team was also helpful in providing identification data for the tagged whales.

Funding Agencies: Massachusetts Environmental Trust, Office of Naval Research, Cecil and Ida Green Technology Award, The International Fund for Animal Welfare, National Marine Fisheries Service Data collection/analysis:

International Fund for Animal Welfare - Song of the Whale Crew (S. Brown, T. Lewis, J. Matthews, R. McLanaghan, A. Moscrop and others). New England Aquarium Right Whale Research (H. Pettis, J. Ciano, L. Conger, P. Hamilton, S. Kraus, A. Knowlton, S. Martin, B.Pike) The WHOI DTAG team (D. Allen, N. Biassoni, C. Carson, M. Johnson, D. Nowacek, J. Partan, A. Shorter, P. Tyack, D. Waples) 